\title{
Lithologic Descriptions of Piston Cores from Chesapeake Bay, Maryland
}

by

\author{
Randall T. Kerhin ${ }^{1}$, Christopher Williams ${ }^{1}$, and Thomas M. Cronin ${ }^{2}$ \\ Maryland Geological Survey, Baltimore, MD \\ U.S. Geological Survey, Reston, VA
}

Open-File Report

98-787

This report is preliminary and has not been reviewed for conformity with U.S. Geological Survey editorial standards or with the North American Stratigraphic Code. Any use of trade, product or firm names is for descriptive purposes only and does not imply endorsement by the U.S. government 


\section{Lithologic Description of Selected Piston Cores \\ Chesapeake Bay, Maryland}

\section{INTRODUCTION}

The U.S Geological Survey is conducting research investigations to understand the nutrient and sediment history of Chesapeake Bay over various time scales and to determine the impact of nutrients and sediment history on living resources. To do this, U.S. Geological Survey will process and analyze various ecological indicators obtained by the sediments.

The Maryland Geological Survey, Resources Assessment Service of the Maryland Department of Natural Resources enter into a cooperative agreement to assist the U.S. Geological Survey in their Critical Ecosystem Program. This cooperative agreement provides support to the Maryland Geological Survey to conduct sediment coring and lithologic core description in Chesapeake Bay in conjunction with U.S. Geological Survey Critical Ecosystem Program to further the understanding of Chesapeake Bay ecosystems.

The objective of this proposal is to obtain continuous sequences of sediments from the Holocene interval of the Chesapeake Bay which consists of the stratigraphic units that fill the Cape Charles paleochannel in the Maryland portion of the Bay.

\section{PRESENT DAY MORPHOLOGY}

The Chesapeake Bay, the largest estuary in the United States, is a classic coastal plain estuary located on a trailing edge continental margin. It is nearly $300 \mathrm{~km}$ long and ranges from 8 to 48 $\mathrm{km}$ wide, having a surface area of almost 6000 square $\mathrm{km}$. The modern Bay formed in response to changing sea level during and following the last major continental glaciation. This history of formation is reflected in the bathymetry, which is characterized by a deep axial channel flanked by broad shallow benches, and in the deeply dissected and invaginated shoreline. Water depths exceed $30 \mathrm{~m}$ in the deep channel but overall the Bay is quite shallow with a average depth of $8 \mathrm{~m}$.

The drainage basin encompasses 167,000 square $\mathrm{km}$ with the Susquehanna River, discharging at the head of the Bay, supplying nearly $50 \%$ of the total fresh water input. The Bay is a microtidal system with a tide range of one meter at the mouth, decreasing progressively up-bay to a minimum of less than 30 centimeters in the vicinity of Baltimore, and then increasing to nearly 60 centimeters at the more constricted head of the Bay. Surface salinities range from near zero at the head of the Bay to near $30 \mathrm{ppt}$ at the mouth. The turbidity maximum, located slightly downstream of the landward limit of saline water intrusion, is generally found $30 \mathrm{~km}$ from the mouth of the Susquehanna River, although its location varies seasonally with the river discharge.

\section{TEXTURAL CHARACTERISTICS OF BOTTOM SEDIMENTS}

Ever since the reconnaissance surveys in the 1950s, the bottom sediments of the Chesapeake Bay have been considered to consist largely of fine grained muds. This assumption prevailed, in part, due to the recognition that the Bay is a microtidal system, has limited fetch inhibiting the 
formation of large wind waves, and receives a large total suspended sediment load from upland drainage sources. In fact, one often encounters direct references to the muddy nature of the Bay's sediments in the literature (eg. "Most of the Bay is blanketed with mud. Sand is predominant only... [in limited areas]. While this assumption is certainly true to a first approximation recent surficial sediment sampling has shown that a considerable sand sized component is present in the Bay bottom sediments and, furthermore, that muddy sediments do not necessarily form a uniform blanket on the Bay floor.

In these studies, (Byrne, et.al., 1983; Kerhin, et.al., 1983) stations were located on a $1 \mathrm{~km}$ grid in Maryland waters and a $1.4 \mathrm{~km}$ offset grid in Virginia waters. At each of the 5924 stations the top $5 \mathrm{~cm}$ of sediment was collected and subjected to a complete grain size analysis over the interval of -1 phi to 10 phi (coarse sand to clay). A settling tube was utilized for analysis of the sand sized component and a Coulter Counter for the silts and clay

\section{SEDIMENT DISTRIBUTION}

The sediment distribution map clearly illustrates the dominance of sand and silty clay in the Maryland and sand in Virginia part of the Bay. The sands are generally located adjacent to the shoreline and on large shelves around the peninsulas and islands of the Eastern Shore. In most areas the sand directly abuts the silty clay, and sediment populations intermediate to the two end-member populations are absent. These mixed sediment types are generally found as isolated pockets surrounded by either sand or silty clay and not as narrow zones between these two dominant sediment populations.

The sand end member represents high energy wave environments in which movement of materials occurs by traction or saltation along the bed surface. Owing to the energy impinging upon the sediment bed, finer material is either not deposited or is actively removed from the sediments. In contrast, the silty clay end member represents deposition of fine materials from suspension in lower energy environments into which sand-sized materials cannot be carried.

Sediments that plot across the center of the diagram from sand to the finer silty clay are generally assumed to represent a gradual decrease of energy. The zone of mixed sediment apparently reflects the gradual decrease of energy associated with the transition from traction to suspension transport. These mixed sediments may also result from alternating high and low energy events creating intercalated sand and silty clay layers that are subsequently reworked by biogenic processes. In either case a zone of "mixed" sediment types would occur separating the sand and silty clay. The two exceptions are the extreme northern Bay and the Choptank River mouth where the dominant sediments are clayey silts, adjacent to the nearshore sands. In both areas, the accumulation of clayey silts reflects a decreasing-energy regime, local geology, and sediment availability.

Mixed sediments occur as isolated pockets located in the midst of larger sand or silty clay fields. Evidence suggests that many of these pockets represent subaqueous exposures of pre-Holocene deposits, particularly in areas where current or wave activity is sufficiently high to prevent accumulation of modern sediments. Because the textural characteristics of these 
sediments do not reflect modern processes, environmental interpretations based upon their grain-size characteristics would be misleading. Of course, anthropogenic activities, particularly dredging and spoil disposal, are not discounted as a possible cause for development of mixed sediments.

Tabulation of the areal extent of sediment types also shows the dominance of the sand and silty clay types. These two sediment types cover over 80 percent of the Bay floor. Silt is the least extensive in the Bay, all silts together covering only 194 square kilometers - less than 7 percent of the Bay. The "mixed" sediment types range from a low of 0.1 percent for sandy clays to a high of approximately 7 percent for the clayey silts.

\section{SEDIMENTATION RATES}

In recent years the determination of sedimentation rates in the estuarine environment has become an increasingly important source of data for calculating sediment budgets, determining the fluxes of anthropogenic materials between the sediments and water column, and identifying potential sites of accumulation of toxic materials. Sedimentation rate information is also important from a geologic perspective, because it has often been stated that estuaries are short lived, rapidly filling with sediments once they are formed. In order to adequately describe the resulting stratigraphy and characteristics of the estuarine sedimentary fill the spatial variations in accumulation rates must be established.

In the Chesapeake Bay, as in most estuaries, sedimentation rates have been determined by radionuclide dating of sediment cores returned from the bottom. In the Maryland portion of the $\mathrm{Bay} \mathrm{Pb}-210$ rates have been obtained by three separate research groups at 16 locations. The rates range from a low of $0.7 \mathrm{~mm} / \mathrm{yr}$ to a high of $80 \mathrm{~mm} / \mathrm{yr}$. Rates determined by this method assume that the sedimentation rate is relatively constant with no periods of erosion or non-deposition, that no remobilization of the radionuclide has occurred, and that the sediment has not been reworked by either physical processes such as slumping or biogenic activity. Radionuclide derived sedimentation rates can only be determined for fine grained sediments which sorb the isotope, and results cannot be extrapolated to adjacent sediments which contain little or no silt and clay sized particles. Because sandy bottom sediments are areally extensive in the Chesapeake Bay the calculated rates are not valid for much of the system.

Some generalizations may be made. The rate of sediment accumulation tends to be higher in the northern portion of the Bay, north of Kent Island, and close to the Potomac River. In the central portion the lowest rates are observed. Also, rates have a tendency to be higher in the axial channel than on the adjacent channel flanks. However, the range of values observed even between cores located close together indicates that in this highly heterogeneous system extrapolation of the results from an individual core over wide areas of the estuary is risky.

In an attempt to overcome the limitations inherent in the radionuclide methodology sedimentation rates for the mainstem of the Bay have been determined by the method of bathymetric comparisons. Using the original survey sheets produced by the National Oceanographic and Atmospheric Administration water depths were averaged for the earliest 
(circa 1850) and the most recent (circa 1950) surveys for which was an adequate density of data points. The results denote the changes in the height of the water column over the time interval spanning the two surveys. The data were rectified to the same mean low water datum by applying correction factors of eustatic sea-level rise $(1 \mathrm{~mm} / \mathrm{yr})$ and an estimate of recent crustal warping for the Bay region. The latter ranged from over $2.6 \mathrm{~mm} / \mathrm{yr}$ subsidence in the northern portion of the Bay to $1.0 \mathrm{~mm} / \mathrm{yr}$ near the Bay mouth.

The bathymetric comparison technique provides an better overview of the sedimentation rates than the nuclide dating because of its ability to provide measurements in the sandy sediments of the Bay, and the capability of developing rate estimates which are representative of large areas of the Bay floor. Due to the nature of the data available from the original sources the Bay was divided into 12 segments based upon basin morphology and bathymetry with the averaged sedimentation rate determined within each segment. In Maryland the average rate of accumulation per year was calculated for both the muddy and sandy sediments within each segment. In Virginia only an average sedimentation rate could be calculated for each segment due to the characteristics of the original data set. The averaged rates shown on Figure 6 are highest in the northern portion of the Bay decline to a low in the upper middle reaches and rise again in the vicinity of the Bay mouth.

The highest sedimentation rate of nearly $0.8 \mathrm{~cm} / \mathrm{yr}$ occurs in the muddy sediments of the northernmost segment of the Bay. In this area suspended sediments supplied by the Susquehanna River are rapidly deposited on the Bay bottom forming the mixed sand-silt-clays and clayey silts. Rates decline southward through segments 2 and 3 to a minimum approaching zero in the upper middle Bay (segment 4). The sediment distribution map reflects this trend with a broad expanse of silty clays present in segments 2 and 3 where sedimentation rates are still relatively high. In segment 4 where rates are low the sediment distribution begins to show a more discontinuous and patchy nature with many isolated pockets of relatively coarse bottom sediments located in deep waters where little modern sediment is accumulating. Rates rise again in segments 5 and 6, though the sediment distribution map continues to show many isolated and discontinuous sediment types. This suggests that the sites of sediment accumulation are localized in this area. Southward through segment 7 to the Bay mouth rates are variable but remain high reflecting the increasing influence of both the Bay mouth sediment source and resuspension due to tidal currents and wind driven waves in an area where the Bay is relatively wide.

\section{SOURCES OF SEDIMENTS}

The sources of sediment to an estuary can be delivered by several different processes: fluvial input mainly as suspended sediment; shoreline erosion; atmospheric dry fall; primary production; erosion of estuarine bottom; and human activity. There is a considerable diversity of information for evaluating these sources of sediments, except for the input of atmospheric dry fall and primary production. However, most discussions of nonpoint sources for sediments are limited to analysis of the inorganic fraction of the total sediment system.

Because the Susquelianna River is the dominant source of fresh water to the Bay, 
numerous studies have investigated the input of the suspended sediment load of the Susquehanna River to northern Bay. The estimates of "normal" suspended sediment discharge of the Susquehanna River has varied from a low of 0.6 million metric tons per year to 1.8 million metric ton per year. Major flooding events completely change the character of suspended sediment discharge in the northern Bay. In 1972, Tropical Storm Agnes flood waters discharge an estimated 31 million metric ton of sediment during that events.

\section{BOTTOM SEDIMENT SAMPLING}

\section{Field}

Aboard the Research Vessel Discovery, fourteen sites in the main Chesapeake Bay were visited in 1996 and 1997 (Table 1). Ten sites were located at major tributaries to the Bay: Rappahannock River in Virginia and the, Potomac, Patuxent and Sassafras Rivers in Maryland. One core site was situated inside the tributary, one at the tributary mouth and one in the main stem of the Bay offshore of the tributary mouth. In the Rappahannock and Sassafras Rivers, the tributary mouth sites were eliminated. Four additional sites were located along a transect from Parker Creek to the Little Choptank River. Two sites near the Parker Creek paleochannel, one in the main channel of the Bay and one in the Little Choptank River. All coring sites were located using Differential Global Positioning System (DGPS).

Short 150 centimeters core samples of the bottom sediment were collected in $6.7 \mathrm{~cm}$ diameter cellulose acetate butyrate $(\mathrm{CAB})$ core liners inserted into a Benthos open-barrel gravity corer. The recovered cores were trimmed at the sediment-water interface, capped, and given to the USGS for microfossil analyses. Longer 4-5 meters core samples were collected with a modified Benthos piston core using a standard $6.7 \mathrm{~cm}$ diameter cellulose acetate butyrate (CAB) core liner attached with a core catcher and brass cutting shoe. The recovered cores were trimmed at 1.5 meter intervals, measured from the bottom of the core and at the sediment-water interface. The core sections were returned to the laboratory for analyses of physical and granulometric characteristics (Table 2).

\section{Laboratory}

In the laboratory, the sediment cores were first X-rayed in their liners with the prints developed using a Xerox 125 xeroradiograph processor. X-rays of cores facilitated recognition of small-scale internal structures, such as clam and worm burrows or tubes, shells, and gas voids. These observations were used to evaluate benthic activity and identify the pre-disposal bottom. On a negative xeroradiographic print, less dense material, such as burrows or gas voids appear darker as compared to denser material, such as shells or sand, which appear lighter.

After the cores were X-rayed, the sediment was extruded from the core liner, split along the axis, photographed, examined, and described. One-half of the piston core was wrapped in plastic wrap for detailed sampling by USGS personnel. The second half of the piston core was scanned for colormetric analysis (Hill and Conkwright, in prep) and logged for physical characteristics and sedimentary structures.

Visual inspection of sediment cores from the Chesapeake Bay shows a broad range of 
colors. Varying hues of brown, greenish and bluish grays, gray, and black can be seen. A typical core exhibits a thin top layer, usually brown or light gray, rapidly becoming dark to nearly black, then gradually lightening to some consistent shade of gray. Some cores display alternating light and dark bands. These color bands range between a few millimeters to several centimeters thick. Other cores show irregular patterns such as mottling, and vermiform or lenticular shaped areas.

Table 1 Geographic Coordinates of Piston and Gravity Cores

\begin{tabular}{|c|c|c|c|c|c|}
\hline River Name & Core ID & $\begin{array}{l}\text { Latitude } \\
\text { NAD } 83\end{array}$ & $\begin{array}{l}\text { Longitude } \\
\text { NAD } 83\end{array}$ & $\begin{array}{r}\text { Water Depth } \\
\text { Meters }\end{array}$ & Date \\
\hline Potomac & PTMC 2-1 & 375824.0 & 761344.0 & 13.20 & $9 / 17 / 96$ \\
\hline Potomac & PTMC 2-2 & 375824.0 & 761344.0 & 13.20 & $9 / 19 / 96$ \\
\hline Potomac & PTMC 3-1 & 38140.20 & 761312.7 & 23.10 & $9 / 25 / 96$ \\
\hline Potomac & PTMC 3-G & 38140.10 & 761312.7 & 20.00 & $9 / 19 / 96$ \\
\hline Potomac & PTMC 3-2 & 38136.70 & 761311.6 & 23.10 & $9 / 25 / 96$ \\
\hline Potomac & PTMC 3-G-2 & 38136.70 & 761311.6 & 24.10 & $9 / 25 / 96$ \\
\hline Parker Creek & PRCK 3-1 & 383238.0 & 762537.1 & 24.30 & $10 / 9 / 96$ \\
\hline Parker Creek & PRCK 3-2 & 383238.0 & 762537.1 & 24.30 & $10 / 9 / 96$ \\
\hline Parker Creek & PRCK 1-1 & 383251.9 & 762942.7 & 10.70 & $9 / 23 / 96$ \\
\hline Parker Creek & PRCK 1-2 & 383251.9 & 762942.7 & 10.70 & $9 / 23 / 96$ \\
\hline Parker Creek & PRCK 2-1 & 38339.30 & 762734.4 & 11.40 & $5 / 7 / 97$ \\
\hline Patuxent & PTXT 1-1 & 381834.9 & 762712.5 & 16.50 & 9/18/96 \\
\hline Patuxent & PTXT 1-2 & 381834.9 & 762712.5 & 16.50 & $9 / 18 / 96$ \\
\hline Patuxent & PTXT 1-3 & 381834.9 & 762712.5 & 16.50 & $9 / 18 / 96$ \\
\hline Patuxent & PTXT 1-G & 381834.9 & 762711.4 & 16.30 & $9 / 19 / 96$ \\
\hline Patuxent & PTXT 2-1 & 381935.0 & 762332.9 & 10.00 & $9 / 18 / 96$ \\
\hline Patuxent & PTXT 2-2 & 381934.4 & 762332.9 & 10.00 & $9 / 18 / 96$ \\
\hline Patuxent & PTXT 2-3 & 381934.7 & 762333.1 & 11.50 & $9 / 18 / 96$ \\
\hline Patuxent & PTXT 2-G & 381935.1 & 762332.8 & 11.40 & $9 / 19 / 96$ \\
\hline Patuxent & PTXT 2-G-2 & 381934.7 & 762333.1 & 11.50 & 9/20/96 \\
\hline Patuxent & PTXT 3-1 & 38200.00 & 761834.8 & 22.50 & $9 / 20 / 96$ \\
\hline Patuxent & PTXT 3-2 & 38200.00 & 761834.8 & 22.50 & $9 / 20 / 96$ \\
\hline Patuxent & PTXT 3-G & 38200.00 & 761834.8 & 22.50 & $9 / 20 / 96$ \\
\hline
\end{tabular}




\begin{tabular}{|llllll|}
\hline River Name & Core ID & $\begin{array}{l}\text { Latitude } \\
\text { NAD 83 }\end{array}$ & $\begin{array}{l}\text { Longitude } \\
\text { NAD 83 }\end{array}$ & $\begin{array}{c}\text { Water Depth } \\
\text { Meters }\end{array}$ & Date \\
\hline Parker Creek & PRCK 1-G & 383251.9 & 762842.7 & 10.70 & $9 / 26 / 96$ \\
Parker Creek & PRCK 2-G & 38339.30 & 762734.4 & 11.40 & $9 / 26 / 96$ \\
Parker Creek & PRCK 3-G & 383238.2 & 762537.2 & 24.30 & $9 / 26 / 96$ \\
Little Choptank & LCPTK 1-1 & 383129.5 & 761811.9 & 13.90 & $9 / 26 / 96$ \\
\hline Little Choptank & LCPTK 1-2 & 383129.5 & 761811.9 & 13.90 & $9 / 26 / 96$ \\
Little Choptank & LCPTK 1-G & 383129.5 & 761811.9 & 13.90 & $9 / 26 / 96$ \\
Rappahannock & RAPRV 1-1 & 373553.3 & 761827.6 & 19.70 & $9 / 24 / 96$ \\
Rappahannock & RAPRV 1-2 & 373553.3 & 761827.6 & 19.70 & $9 / 24 / 96$ \\
Rappahannock & RAPRV 1-G & 373553.3 & 761827.6 & 19.70 & $9 / 24 / 96$ \\
Rappahannock & RAPRV 2-1 & 373228.2 & 761021.9 & 11.50 & $9 / 24 / 96$ \\
Rappahannock & RAPRV 2-2 & 373228.2 & 761021.9 & 11.50 & $9 / 24 / 96$ \\
Rappahannock & RAPRV 2-G & 373228.2 & 761021.9 & 11.50 & $9 / 24 / 96$ \\
Potomac & PTMC 1-1 & 38150.30 & 762240.6 & 19.00 & $9 / 19 / 96$ \\
Potomac & PTMC 1-2 & 38150.30 & 762240.6 & 19.00 & $9 / 19 / 96$ \\
Potomac & PTMC 1-G & 38150.30 & 762240.6 & 19.00 & $9 / 17 / 96$ \\
\hline
\end{tabular}


Table 2 Piston Core Lengths

\begin{tabular}{|llllll|}
\hline River Name & Core ID & $\begin{array}{c}\text { Total } \\
\text { Length }\end{array}$ & Top & Middle & Bottom \\
\hline Patuxent & PTXT 1-3 & 199 & 75 & 124 & 0 \\
Patuxent & PTXT 2-3 & 417 & 110 & 153 & 154 \\
Patuxent & PTXT 3-2 & 432 & 133 & 153 & 146 \\
Potomac & PTMC 3-2 & 452 & 150 & 152 & 150 \\
Potomac & PTMC 2-2 & 354 & 57 & 150 & 147 \\
Potomac & PTMC 1-2 & 422 & 122 & 150 & 150 \\
Rappahannock & RAPRV 1-2 & 232 & 86 & 146 & 0 \\
Rappahannock & RAPRV 2-2 & 405 & 109 & 150 & 146 \\
Rappahannock & RAPRV 3-2 & 452 & 145 & 154 & 153 \\
Little Choptank & LCPTK 1-1 & 455 & 147 & 153 & 155 \\
Parker Creek & PRCK 1-2 & 315 & 107 & 77 & 131 \\
Parker Creek & PRCK 3-2 & 452 & 145 & 154 & 153 \\
Sassafras River & SASS 1-1 & 407 & 109 & 151 & 147 \\
Sassafras River & SASS 2-2 & 383 & 95 & 153 & 134 \\
Parker Creek & PRCK 2-1 & 426 & 120 & 153 & 153 \\
\hline
\end{tabular}


Grain size, mineralogy, carbon content, organic matter, and water content partially determine the color and intensity of light reflected from sediments. The presence of sulfur minerals greatly affects the color of fine grained, cohesive sediments. Chemical analysis of fine grained, cohesive sediments from the Chesapeake Bay indicate that the major sulfur minerals present are pyrite, monosulfide species and native sulfur. Further analysis shows a distinct relationship between the ratio of pyrite to monosulfides and the color of these sediments. Light gray sediments have a high pyrite to monosulfide ratio, while dark sediments have low pyrite to monosulfide ratio. A linear relationship exists between the pyrite to monosulfide ratio $(\mathrm{p} / \mathrm{m}$ ratio) and the intensity of light reflected from the sediments. By measuring the reflection intensity along the entire length of a carefully cross-sectioned core and chemically analyzing the sulfur mineralogy in a few subsamples, the sulfur mineralogy of the entire core can be determined.

The core scanner is designed to measure the intensity of selected light wavelengths reflected from the surface of sediment cores. The scanner continuously records the reflection intensity of the sediments and the depth down-core of the reflection measurements. Scanner data can be sent to an $\mathrm{X}-\mathrm{Y}$ recorder and/or and analog to digital converter for digital storage.

The sediment was subsequently subsampled and analyzed for water content, porosity, bulk density, and on selected subsamples, grain-size. Datable materials such as shell was also sampled and forwarded to USGS for Carbon 14 dating. Analyses were conducted according to MGS standard techniques as outlined in Kerhin and others (1988). Two homogeneous splits of each sample were processed, one for bulk property analyses and the other for grain-size characterization. Analyses were performed as soon as possible after sample collection, and all samples were refrigerated in sealed Whirl-Pak plastic bags prior to analysis.

Water content was measured at selected intervals from the core upon extrusion from the liner. Samples were divided into weighed $30 \mathrm{~g}$ portions, dried at $65^{\circ} \mathrm{C}$, and then reweighed. Water content was calculated as the percentage of water weight to the total weight of wet sediment, as follows:

$$
\% H_{2} O=\left(\frac{W_{w}}{W_{t}}\right) \times 100
$$

weight of water, and $W_{1}$ is the weight of wet sediment.

where $W_{w}$ is the

The water content may be underestimated because of water being lost from the sediment during the time interval between collection and extrusion of the core. Recently deposited dredged sediments, contained within the core liner, exhibit a measurable amount of dewatering between the time of collection and analysis, resulting in compaction. The amount of water expressed from the sediment subsequent to collection can be calculated by measuring the change in core length prior to extrusion. Water contents calculated in the laboratory were corrected by 
assuming that this compaction occurred evenly throughout the thickness of the most recent dredged sediment layer. The water content values reported for the sampling locations represent the corrected average of the interval samples downcore.

The precision of water content measurements was determined by calculating the relative standard deviation. For sediment samples collected and analyzed in this manner, the relative standard deviation for percent water content was determined to be 4.46 percent (Halka and Panageotou, 1993). The standard deviation $(\sigma)$ for any particular water content may be calculated as:

$$
\mathrm{\sigma H}_{2} \mathrm{O}=\frac{\% \mathrm{H}_{2} \mathrm{O}}{100} \times 4.46
$$

Bulk density $\left(\rho_{\mathrm{b}}\right)$ and porosity $(P)$ were calculated from water content utilizing equations (3) and (4) by assuming an average grain density $\left(\rho_{\mathrm{s}}\right)$ of $2.72 \mathrm{~g} / \mathrm{cm}^{3}$ and saturation of voids with water of density $\rho_{w}=1.0 \mathrm{~g} / \mathrm{cm}^{3}$. This method was adopted from the work of Bennett and Lambert (1971):

$$
\rho_{b}=\frac{W_{t}}{W_{d} / 2.72+W_{w}}
$$

weight of dry sediment.

$$
P=\rho_{s} \frac{\% H_{2} O}{\rho_{s} \% H_{2} O+\rho_{w}\left(1-\% H_{2} O\right)}
$$

The amount of volumetric reduction $\left(\mathrm{V}_{\Delta}\right)$ attributable to in situ dewatering (consolidation) of the deposit was determined by calculating the volume change over time resulting from the reduction in porosity in the collected cores. Porosity was calculated from the average water contents of the cores according to equation (4). Over a period of time the volume change due to consolidation can be calculated utilizing equation (5):

$$
V_{\Delta}=\frac{1-P_{i}}{1-P_{f}} \times 100
$$

where $P_{i}$ is the initial porosity at time one, and $P_{f}$ is the final porosity at time two. The amount of erosion which has occurred can then be estimated by first calculating the total sediment volume change from acoustic reflection or bathymetric analyses, and subtracting the volume determined to be due to in situ dewatering. 
Grain size analysis consisted of cleaning the samples in solutions of 10 percent hydrochloric acid and 15 percent hydrogen peroxide with subsequent rinsing with deionized water. This process removed soluble salts, carbonates, and organic matter that could interfere with the disaggregation of the individual grains. The samples were then treated with a 0.26 percent solution of the dispersant sodium hexametaphosphate $\left(\left(\mathrm{NaPO}_{3}\right)_{6}\right)$ to ensure that individual grains did not reaggregate during analysis.

The separation of sand and silt-clay portions of the sample was accomplished by wetsieving through a 4-phi mesh sieve $(0.0625 \mathrm{~mm}$, U.S. Standard Sieve \#230). The sand fraction was dried and weighed. The finer silt and clay sized particles were suspended in a $1000 \mathrm{ml}$ cylinder in a solution of 0.26 percent sodium hexametaphosphate. The suspension was agitated and, at specified times thereafter, $20 \mathrm{ml}$ pipette withdrawals were made (Carver, 1971; Folk, 1974). The rationale behind this process is that larger particles settle faster than smaller ones. By calculating the settling velocities for different sized particles, times for withdrawal can be determined at which all particles of a specified size will have settled past the point of withdrawal. Sampling times were calculated to permit the determination of the amount of silt (4 phi) and clay sized ( $8 \mathrm{phi}$ ) particles in the suspension. Withdrawn samples were dried at $60^{\circ} \mathrm{C}$ and weighed. From these data the percentages by dry weight of sand, silt, and clay were calculated for each sample and classified according to Shepard's (1954) nomenclature (Figure 3).

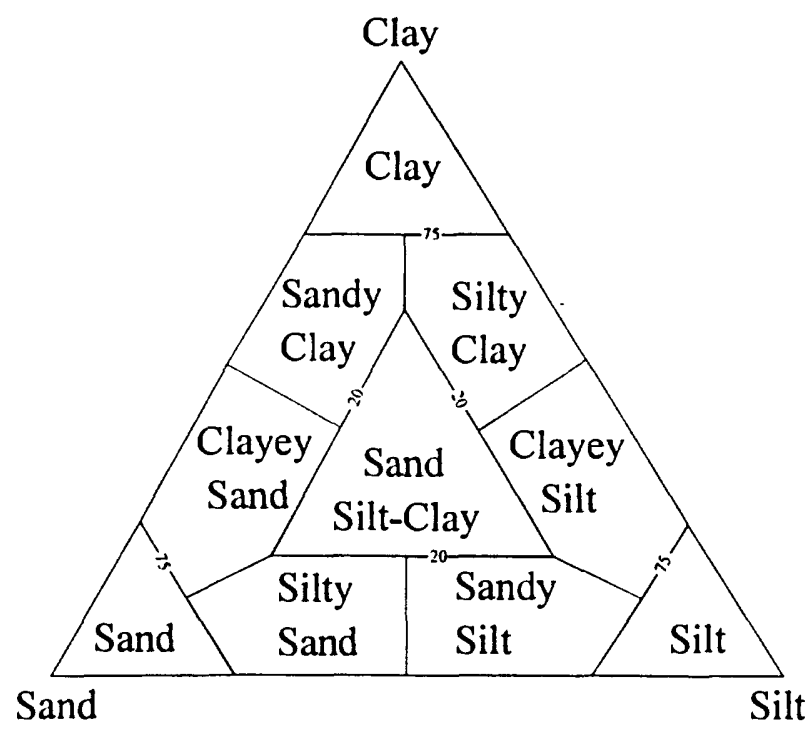

Figure 1. Shepard's (1954) classification of sediment types. 


\begin{tabular}{|c|c|c|c|c|c|c|c|c|c|c|c|c|c|c|c|c|}
\hline$\frac{\stackrel{\sigma}{3}}{\frac{3}{3}}$ & $\frac{\pi}{3}$ & 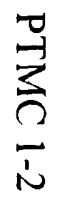 & $\frac{\pi}{3}$ & $\frac{\pi}{3}$ & $\frac{\sqrt{3}}{3}$ & 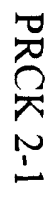 & 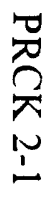 & 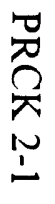 & 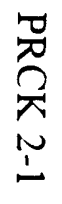 & 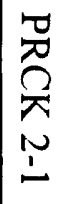 & 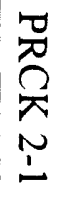 & 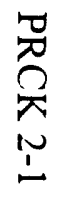 & 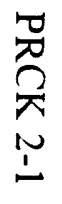 & 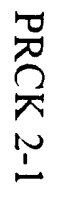 & 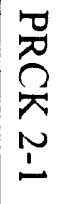 & $\stackrel{\Omega}{\frac{0}{3}}$ \\
\hline 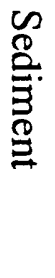 & 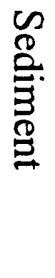 & 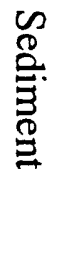 & 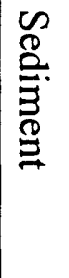 & 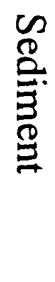 & 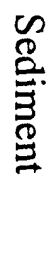 & 芯 & 胥. & 疋 & 疋. & 蔦. & 胥. & 疋 & 㺃. & 胥. & 蛋. & 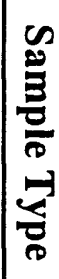 \\
\hline $\begin{array}{l}\vec{\omega} \\
\stackrel{\vec{\omega}}{\underline{\omega}} \\
\stackrel{\omega}{\omega}\end{array}$ & 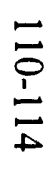 & $\begin{array}{l}\stackrel{D}{\prime} \\
\stackrel{D}{D}\end{array}$ & $\begin{array}{l}\stackrel{P}{0} \\
\stackrel{1}{\phi}\end{array}$ & $\begin{array}{l}\overrightarrow{\hat{a}} \\
\hat{\mathbf{o}}\end{array}$ & $\stackrel{p}{1}$ & 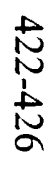 & $\begin{array}{l}\underset{\omega}{\infty} \\
\stackrel{\infty}{0} \\
\dot{\omega} \\
\infty \\
\infty\end{array}$ & 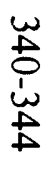 & $\begin{array}{l}w \\
\stackrel{0}{8} \\
\dot{\omega} \\
\stackrel{0}{1}\end{array}$ & $\begin{array}{l}\text { N } \\
\text { N } \\
\tilde{\Omega}\end{array}$ & 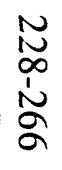 & 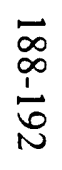 & $\frac{\vec{P}}{\stackrel{+}{+}}$ & $\begin{array}{l}\text { No } \\
\text { ஸे }\end{array}$ & $\begin{array}{l}\vec{a} \\
\text { ì } \\
\text { d. }\end{array}$ & 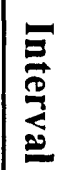 \\
\hline
\end{tabular}

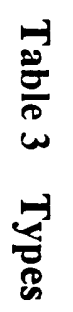

w

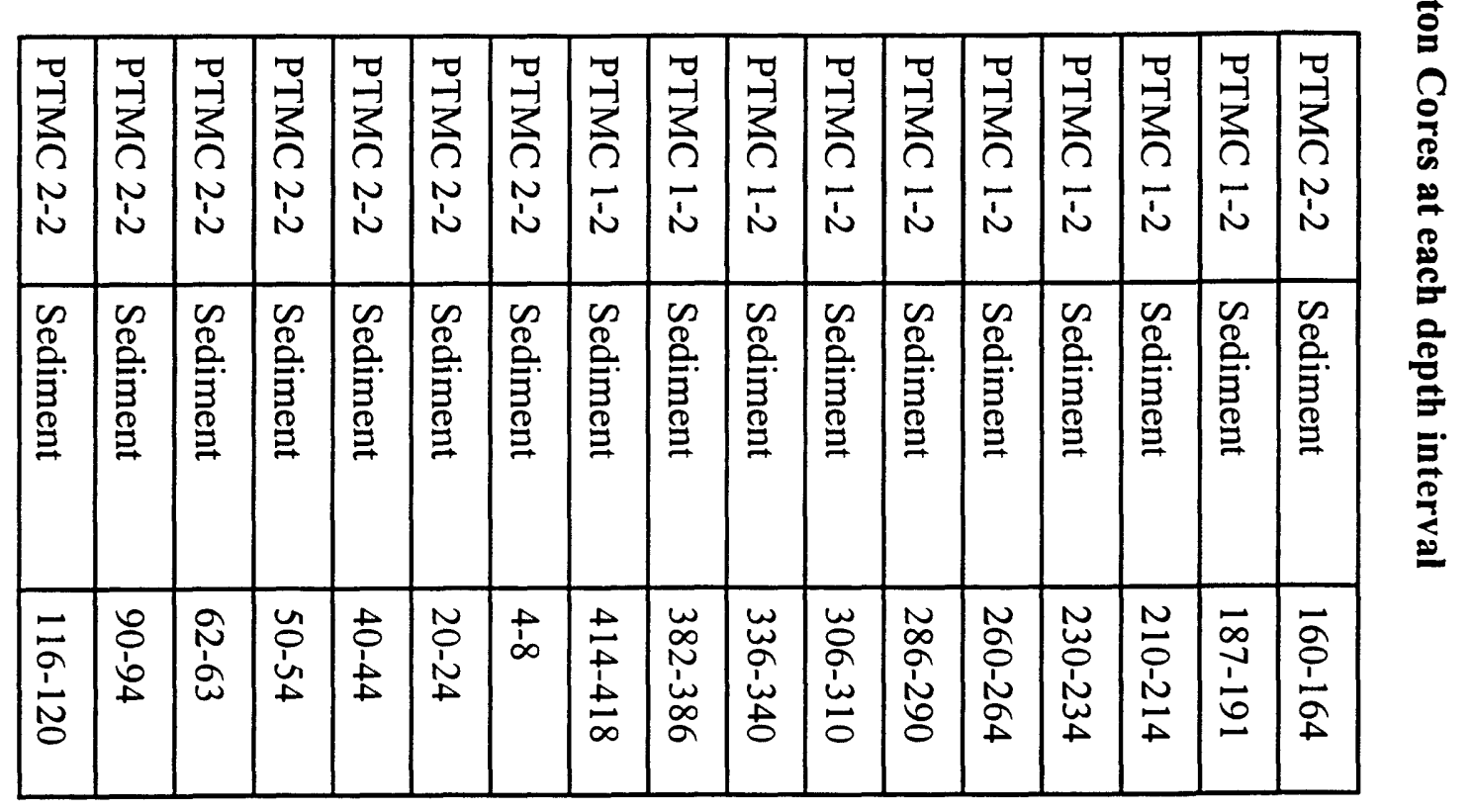




\begin{tabular}{|c|c|c|c|c|c|c|c|c|c|c|c|c|c|c|c|c|c|c|}
\hline 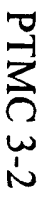 & 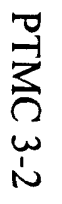 & 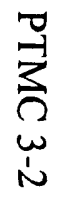 & 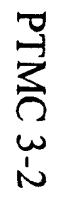 & 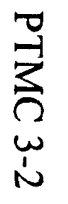 & 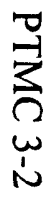 & 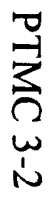 & 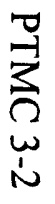 & 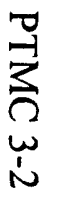 & 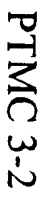 & 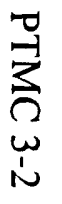 & 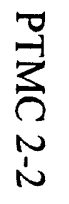 & 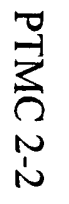 & 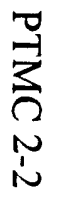 & 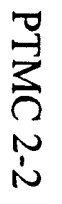 & 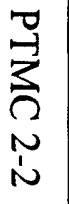 & 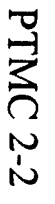 & 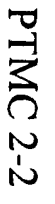 & م \\
\hline 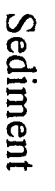 & 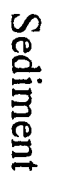 & 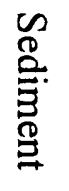 & 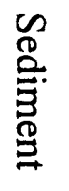 & 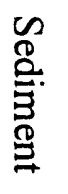 & 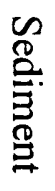 & 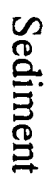 & 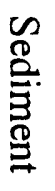 & 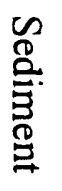 & 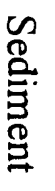 & 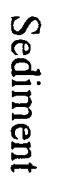 & 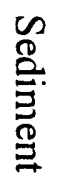 & 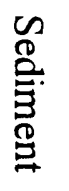 & 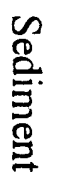 & 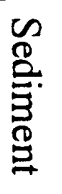 & 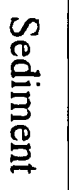 & 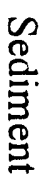 & 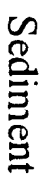 & 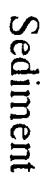 \\
\hline 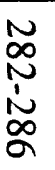 & 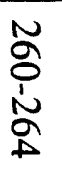 & $\begin{array}{l}\tilde{\omega} \\
\stackrel{1}{1} \\
\tilde{1} \\
0\end{array}$ & $\begin{array}{l}\text { O } \\
\stackrel{1}{\alpha} \\
\stackrel{1}{8}\end{array}$ & $\begin{array}{l}\vec{n} \\
\frac{1}{2} \\
\frac{1}{0}\end{array}$ & $\begin{array}{l}\vec{N} \\
\infty \\
1 \\
\stackrel{\omega}{N}\end{array}$ & $\begin{array}{l}\overrightarrow{8} \\
9 \\
\dot{1} \\
\overrightarrow{0}\end{array}$ & $\begin{array}{l}\text { J人 } \\
\dot{1} \\
\infty \\
0\end{array}$ & $\begin{array}{l}\text { 感 } \\
\dot{1}\end{array}$ & $\begin{array}{l}\underset{O}{N} \\
\stackrel{1}{\Delta}\end{array}$ & $\tilde{a}$ & $\begin{array}{l}\omega \\
\stackrel{\omega}{0} \\
\dot{\omega} \\
\stackrel{\omega}{0}\end{array}$ & $\begin{array}{l}\omega \\
\tilde{\omega} \\
\stackrel{\omega}{\omega} \\
\text { w }\end{array}$ & $\begin{array}{l}\text { No } \\
o \\
\dot{1} \\
\text { ర }\end{array}$ & $\begin{array}{l}N \\
\infty \\
+ \\
+ \\
\infty \\
\infty\end{array}$ & 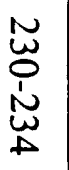 & $\begin{array}{l}\stackrel{N}{\vec{O}} \\
\stackrel{N}{\sim} \\
\stackrel{D}{A}\end{array}$ & $\begin{array}{l}\text { Ш } \\
\stackrel{\infty}{\perp} \\
\stackrel{\infty}{N}\end{array}$ & $\begin{array}{l}\vec{D} \\
\stackrel{N}{1} \\
\frac{1}{\Delta} \\
\stackrel{a}{a}\end{array}$ \\
\hline
\end{tabular}

\begin{tabular}{|c|c|c|c|c|c|c|c|c|c|c|c|c|c|c|c|c|c|c|}
\hline 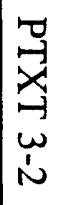 & 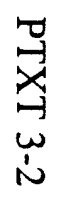 & 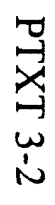 & 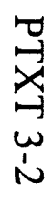 & 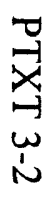 & 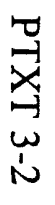 & 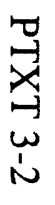 & 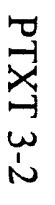 & 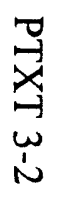 & 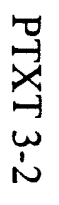 & 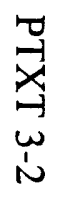 & 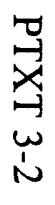 & 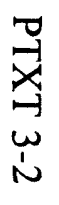 & 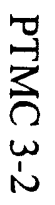 & 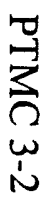 & 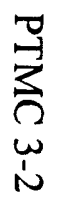 & 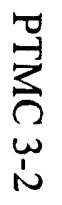 & 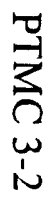 & 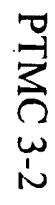 \\
\hline 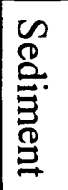 & 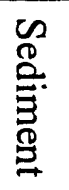 & 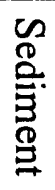 & 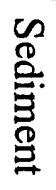 & 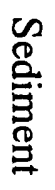 & 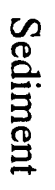 & 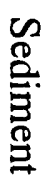 & 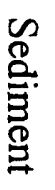 & 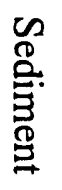 & 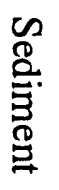 & 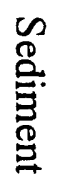 & 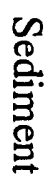 & 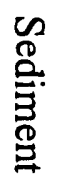 & 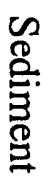 & 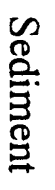 & 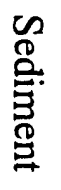 & 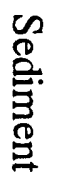 & 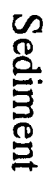 & 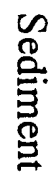 \\
\hline $\begin{array}{l}\stackrel{D}{N} \\
\infty \\
\dot{\omega} \\
\omega\end{array}$ & $\begin{array}{l}\omega \\
\text { w } \\
\text { w } \\
\text { o }\end{array}$ & $\begin{array}{l}w \\
\text { on } \\
\dot{\omega} \\
\text { gे }\end{array}$ & $\begin{array}{l}\underset{w}{w} \\
\text { o } \\
\stackrel{\omega}{\omega} \\
\dot{0}\end{array}$ & 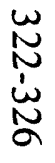 & $\begin{array}{l}\text { N } \\
\text { N } \\
\frac{1}{\sigma}\end{array}$ & $\begin{array}{l}N \\
\stackrel{N}{\infty} \\
1 \\
\sim \\
N\end{array}$ & $\begin{array}{l}N \\
\varnothing \\
\infty \\
N \\
N \\
N\end{array}$ & $\begin{array}{l}\vec{D} \\
\stackrel{1}{D} \\
\vec{D}\end{array}$ & $\frac{N}{\stackrel{N}{N}}$ & $\begin{array}{l}\text { ov } \\
1 \\
0 \\
0\end{array}$ & $\begin{array}{l}\mathbf{w} \\
\stackrel{\omega}{\alpha}\end{array}$ & $\frac{\overrightarrow{0}}{\dot{A}}$ & $\begin{array}{l}\frac{B}{d} \\
\text { D } \\
\dot{t} \\
\stackrel{b}{0}\end{array}$ & $\begin{array}{l} \pm \\
\infty \\
1 \\
\dot{N} \\
N\end{array}$ & $\begin{array}{l}\underset{0}{0} \\
\stackrel{+}{+} \\
\underset{\infty}{\infty}\end{array}$ & $\begin{array}{l}\tilde{w} \\
\mathscr{\alpha} \\
\dot{1} \\
\stackrel{\alpha}{o}\end{array}$ & $\begin{array}{l}\underset{+}{\omega} \\
\underset{\omega}{\omega} \\
\underset{\infty}{w}\end{array}$ & $\begin{array}{l}\omega \\
0 \\
\dot{\omega} \\
\dot{\omega} \\
0\end{array}$ \\
\hline
\end{tabular}




\begin{tabular}{|c|c|c|c|c|c|c|c|c|c|c|c|c|c|c|c|c|c|c|}
\hline 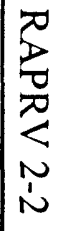 & $\underset{\substack{\infty \\
\infty}}{\infty}$ & 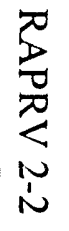 & 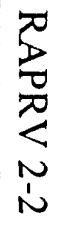 & 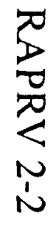 & 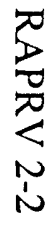 & 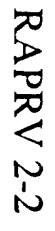 & 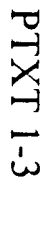 & 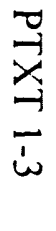 & 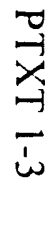 & 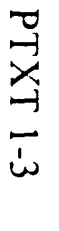 & 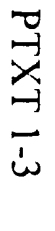 & 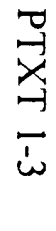 & 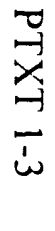 & 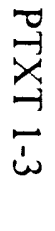 & 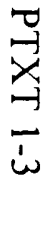 & $\begin{array}{l}\vec{A} \\
\vec{x} \\
\vec{A} \\
\dot{\omega}\end{array}$ & 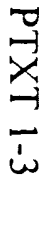 & 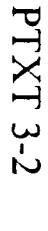 \\
\hline 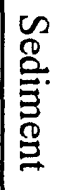 & 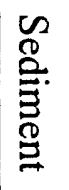 & 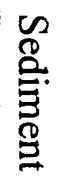 & 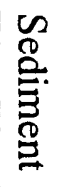 & 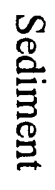 & 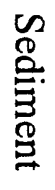 & 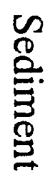 & 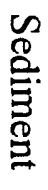 & 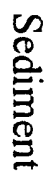 & 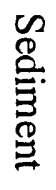 & 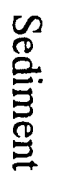 & 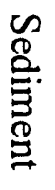 & 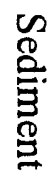 & 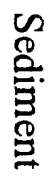 & 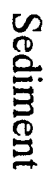 & 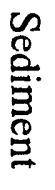 & 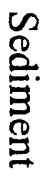 & 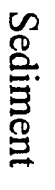 & 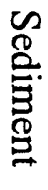 \\
\hline $\begin{array}{l}\tilde{O} \\
8 \\
0 \\
\tilde{1} \\
0 \\
\end{array}$ & $\begin{array}{l}\vec{a} \\
\frac{1}{2} \\
\infty\end{array}$ & $\frac{\tilde{N}}{\stackrel{1}{\omega}}$ & $\begin{array}{l}b \\
0 \\
1 \\
1\end{array}$ & $\underset{\infty}{\stackrel{\omega}{\omega}}$ & $\begin{array}{l}\vec{a} \\
\text { ஸे }\end{array}$ & $\underset{\infty}{+}$ & $\begin{array}{l}\vec{b} \\
\frac{1}{b} \\
b\end{array}$ & $\begin{array}{l}\infty \\
\stackrel{\infty}{+} \\
\frac{\infty}{\infty}\end{array}$ & $\begin{array}{l}\vec{a} \\
\frac{1}{1} \\
\frac{1}{0}\end{array}$ & $\frac{\vec{t}}{\stackrel{+}{+}}$ & $\frac{\bar{N}}{\frac{1}{N}}$ & $\frac{\overrightarrow{8}}{\stackrel{+}{\circ}}$ & $\begin{array}{l}\infty \\
\stackrel{1}{0} \\
0\end{array}$ & $\stackrel{a}{\dot{\phi}}$ & $\begin{array}{l}\frac{\Delta}{N} \\
\dot{1} \\
\hat{a}\end{array}$ & $\underset{\infty}{\stackrel{\omega}{\omega}}$ & $\underset{\infty}{\vec{\infty}}$ & 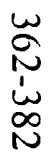 \\
\hline
\end{tabular}

जu

\begin{tabular}{|c|c|c|c|c|c|c|c|c|c|c|c|c|c|c|c|c|c|c|}
\hline 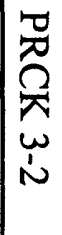 & 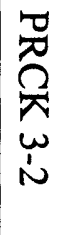 & 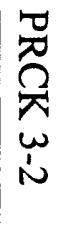 & $\begin{array}{l}D_{0}^{\infty} \\
\frac{0}{2} \\
\frac{1}{2}\end{array}$ & 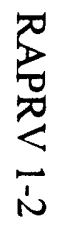 & 茎 & 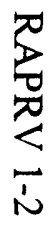 & 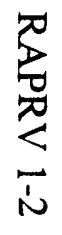 & 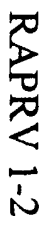 & 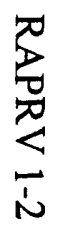 & 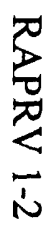 & 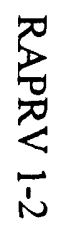 & 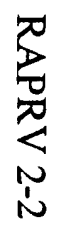 & 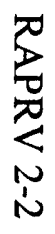 & 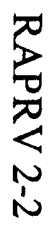 & 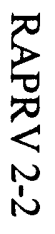 & 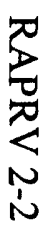 & 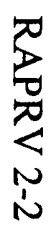 & 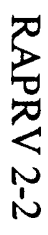 \\
\hline 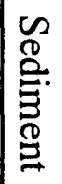 & 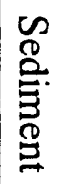 & 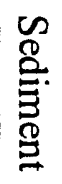 & 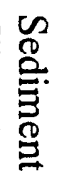 & 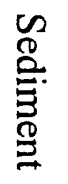 & 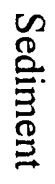 & 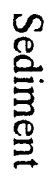 & 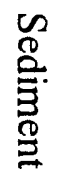 & 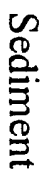 & 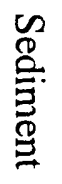 & 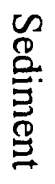 & 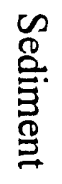 & 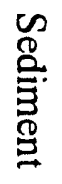 & 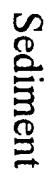 & 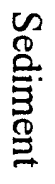 & 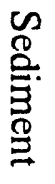 & 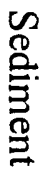 & 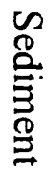 & 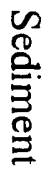 \\
\hline $\begin{array}{l}\checkmark \\
\infty \\
\infty \\
\infty \\
N\end{array}$ & $\begin{array}{l}\text { u } \\
\text { ò }\end{array}$ & $\underset{\dot{\infty}}{\dot{b}}$ & $\begin{array}{l}\text { N } \\
\text { N } \\
\text { N } \\
\text { }\end{array}$ & 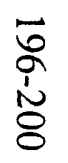 & $\begin{array}{l}\vec{n} \\
\dot{1} \\
\stackrel{n}{n}\end{array}$ & $\frac{\bar{\sigma}}{\frac{1}{0}}$ & $\frac{0}{\frac{1}{8}}$ & 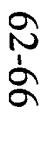 & $\begin{array}{l}\text { N } \\
\stackrel{1}{\Lambda}\end{array}$ & $\frac{\vec{t}}{\infty}$ & $\underline{n}$ & $\begin{array}{l}+ \\
8 \\
\dot{8} \\
+ \\
+\end{array}$ & $\begin{array}{l}\text { w } \\
\text { ô } \\
1 \\
\text { t) }\end{array}$ & $\begin{array}{l}\omega \\
\text { o } \\
\text { o. } \\
\stackrel{\omega}{0} \\
0\end{array}$ & 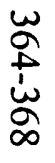 & 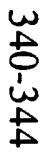 & 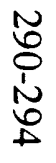 & 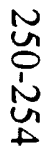 \\
\hline
\end{tabular}




\begin{tabular}{|c|c|c|c|c|c|c|c|c|c|c|c|c|c|c|c|c|c|c|}
\hline 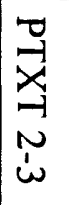 & $\begin{array}{l}\underset{-1}{-1} \\
\stackrel{-1}{x} \\
\stackrel{1}{\omega}\end{array}$ & 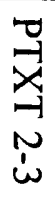 & 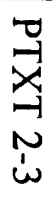 & 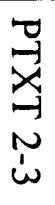 & 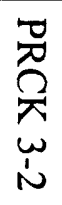 & 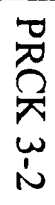 & 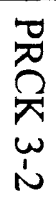 & 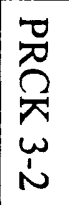 & 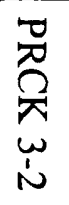 & 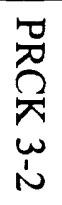 & 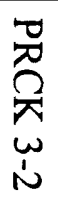 & 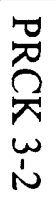 & 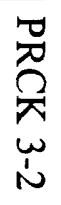 & 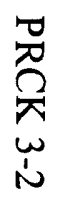 & $\begin{array}{l}\overrightarrow{0} \\
\widetilde{D} \\
\widetilde{\lambda} \\
\omega \\
\dot{\omega}\end{array}$ & 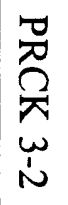 & 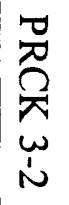 & 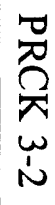 \\
\hline 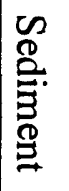 & 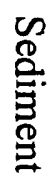 & 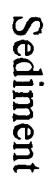 & 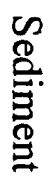 & 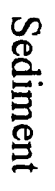 & 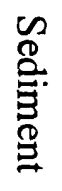 & 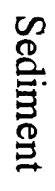 & 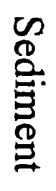 & 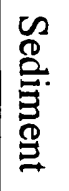 & 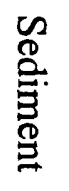 & 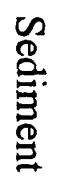 & 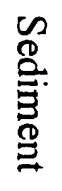 & 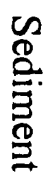 & 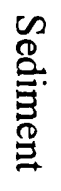 & 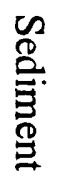 & 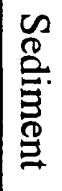 & 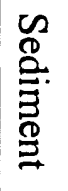 & 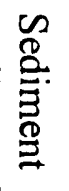 & $\frac{\infty}{\frac{D}{D}}$ \\
\hline$\frac{N}{1}$ & $\begin{array}{l}\text { o } \\
1 \\
\text { N }\end{array}$ & $\begin{array}{l}\stackrel{D}{\infty} \\
\dot{\sim} \\
\dot{N}\end{array}$ & 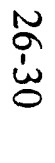 & $\stackrel{+}{\infty}$ & 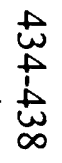 & $\begin{array}{l}\stackrel{\oplus}{\infty} \\
\stackrel{+}{N} \\
\stackrel{N}{N}\end{array}$ & $\begin{array}{l}+ \\
8 \\
1 \\
1 \\
\stackrel{1}{0}\end{array}$ & $\begin{array}{l}\omega \\
\infty \\
\stackrel{\omega}{\omega} \\
\infty \\
+\end{array}$ & 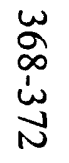 & $\begin{array}{l}w \\
w \\
\alpha \\
\dot{\omega} \\
\dot{0}\end{array}$ & $\begin{array}{l}N \\
\infty \\
\infty \\
\text { N్} \\
\text { N }\end{array}$ & 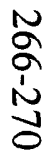 & $\begin{array}{l}N \\
\stackrel{N}{\infty} \\
\stackrel{N}{N} \\
N\end{array}$ & $\begin{array}{l}N \\
\text { N } \\
\text { N } \\
N \\
\mathbb{D}\end{array}$ & 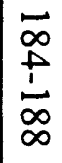 & $\begin{array}{l}\vec{n} \\
\frac{1}{\sigma} \\
\dot{0}\end{array}$ & $\begin{array}{l}\vec{\omega} \\
\infty \\
\stackrel{1}{\Delta} \\
\stackrel{N}{N}\end{array}$ & $\frac{\tilde{N}}{\stackrel{\sim}{N}}$ \\
\hline
\end{tabular}

5

\begin{tabular}{|c|c|c|c|c|c|c|c|c|c|c|c|c|c|c|c|c|c|c|}
\hline 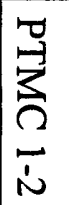 & 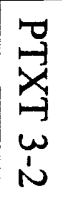 & 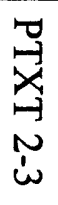 & 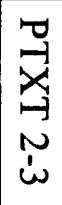 & 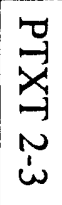 & 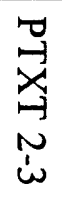 & 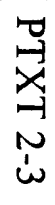 & 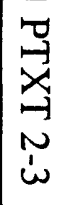 & 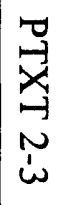 & 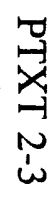 & 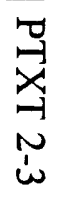 & 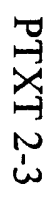 & 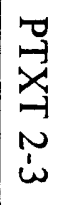 & 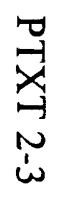 & 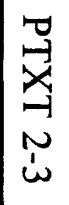 & 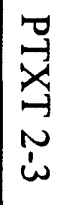 & 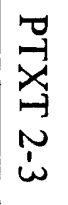 & 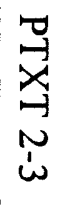 & 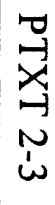 \\
\hline$\frac{\infty}{\frac{\infty}{\sigma}}$ & $\frac{\infty}{\frac{\sigma}{\sigma}}$ & $\frac{\infty}{\frac{\omega}{\sigma}}$ & $\frac{\infty}{\frac{\infty}{\sigma}}$ & 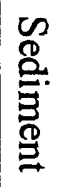 & 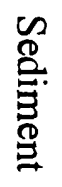 & 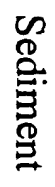 & 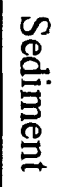 & 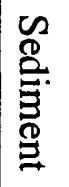 & 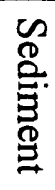 & 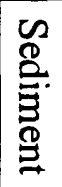 & $\frac{\infty}{\sigma}$ & 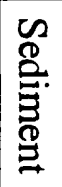 & 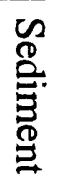 & 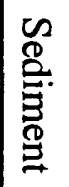 & 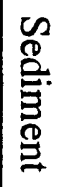 & 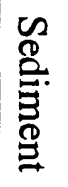 & 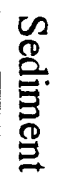 & 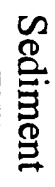 \\
\hline 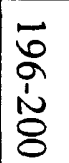 & 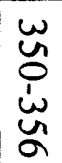 & $\begin{array}{l}\vec{b} \\
80 \\
\stackrel{b}{t} \\
\omega\end{array}$ & $\begin{array}{l}\omega \\
\stackrel{\omega}{\omega} \\
\dot{\omega} \\
\stackrel{\Delta}{\omega}\end{array}$ & $\begin{array}{l}\stackrel{+}{0} \\
\text { on } \\
\dot{1} \\
\text { \& }\end{array}$ & 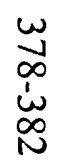 & $\begin{array}{l}\omega \\
\underset{\infty}{\infty} \\
\dot{\omega} \\
\underset{N}{N}\end{array}$ & 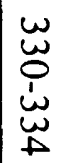 & $\begin{array}{l}\omega \\
\stackrel{\omega}{8} \\
\dot{\phi} \\
\dot{\omega} \\
\varnothing\end{array}$ & 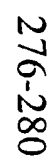 & $\begin{array}{l}\text { N } \\
\text { N } \\
\text { N } \\
\text { ă }\end{array}$ & $\begin{array}{l}\text { N } \\
+ \\
\text { N } \\
\text { N }\end{array}$ & $\begin{array}{l}\text { N } \\
\stackrel{+}{N} \\
\text { N } \\
\text { 去 }\end{array}$ & $\begin{array}{l}\underset{\infty}{N} \\
\stackrel{N}{N} \\
\stackrel{\mathbb{N}}{N}\end{array}$ & $\begin{array}{c}\sim \\
\omega \\
+ \\
\sim \\
\omega\end{array}$ & $\frac{N}{\stackrel{N}{N}}$ & $\frac{\bar{\Omega}}{\frac{1}{1}}$ & $\begin{array}{l}\bar{\omega} \\
\frac{1}{\omega} \\
\tilde{a}\end{array}$ & $\begin{array}{l}\infty \\
\infty \\
1 \\
0 \\
0\end{array}$ \\
\hline
\end{tabular}




\begin{tabular}{|c|c|c|c|c|c|c|c|c|c|c|c|c|c|c|c|c|c|c|}
\hline 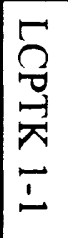 & 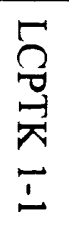 & 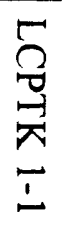 & 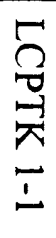 & 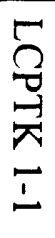 & 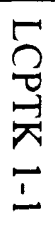 & 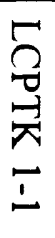 & $\begin{array}{l}5 \\
\overrightarrow{-1} \\
\frac{-1}{2} \\
-1\end{array}$ & 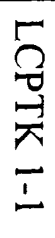 & 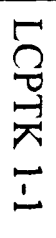 & $\begin{array}{l}\vec{\partial} \\
\overrightarrow{-} \\
\vec{\lambda} \\
\vec{\sim}\end{array}$ & 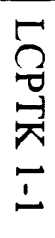 & $\begin{array}{l}\underset{2}{2} \\
\stackrel{-}{二} \\
\stackrel{1}{-}\end{array}$ & 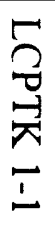 & 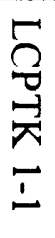 & 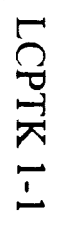 & 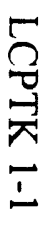 & 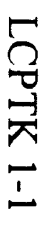 & 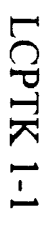 \\
\hline 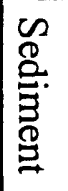 & 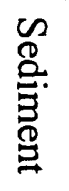 & $\frac{\infty}{\overrightarrow{0}}$ & 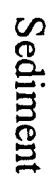 & 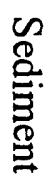 & 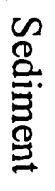 & 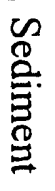 & 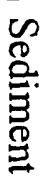 & 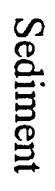 & 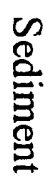 & 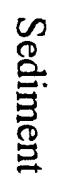 & 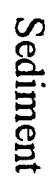 & 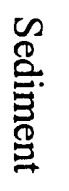 & 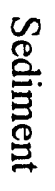 & 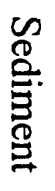 & 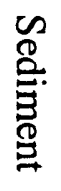 & 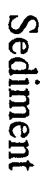 & 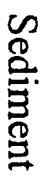 & 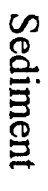 \\
\hline 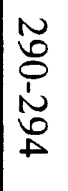 & $\begin{array}{l}\text { N } \\
\text { ஸ́ } \\
\text { 心ু }\end{array}$ & $\begin{array}{l}\text { N } \\
\text { O } \\
\text { N } \\
\text { N }\end{array}$ & $\begin{array}{l}\text { N } \\
\stackrel{+}{0} \\
\stackrel{N}{\sim} \\
\stackrel{+}{A}\end{array}$ & $\begin{array}{l}\stackrel{N}{\sim} \\
\stackrel{\sim}{\sim} \\
\stackrel{\sim}{\sim}\end{array}$ & $\begin{array}{l}\stackrel{\sim}{\omega} \\
\stackrel{\omega}{\sim} \\
\stackrel{\sim}{\sim}\end{array}$ & $\begin{array}{l}\stackrel{\sim}{\Xi} \\
\stackrel{\sim}{\omega} \\
\stackrel{\omega}{\omega}\end{array}$ & $\begin{array}{l}\dot{8} \\
\frac{1}{6}\end{array}$ & $\begin{array}{l}\bar{D} \\
\dot{0} \\
\dot{1} \\
\stackrel{1}{D}\end{array}$ & 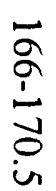 & $\begin{array}{l}\vec{w} \\
\infty \\
\frac{1}{\Delta} \\
\vec{N}\end{array}$ & $\begin{array}{l}\bar{\omega} \\
\stackrel{1}{1} \\
\tilde{\omega}\end{array}$ & 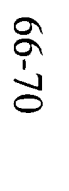 & $\begin{array}{l}\text { gे } \\
\text { ó } \\
\text { ó }\end{array}$ & $\underset{\infty}{+}$ & $\underset{\infty}{\stackrel{\omega}{+}}$ & $\begin{array}{l}\text { N } \\
\stackrel{\infty}{\omega} \\
\stackrel{N}{N}\end{array}$ & $\begin{array}{l}\vec{a} \\
\dot{1} \\
0\end{array}$ & $\stackrel{t}{\infty}$ \\
\hline
\end{tabular}

$\nabla$

\begin{tabular}{|c|c|c|c|c|c|c|c|c|c|c|c|c|c|c|c|c|c|c|}
\hline 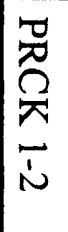 & 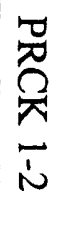 & 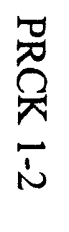 & 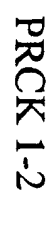 & 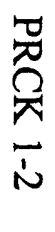 & 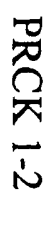 & 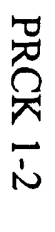 & 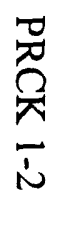 & 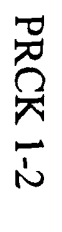 & 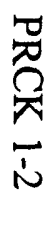 & 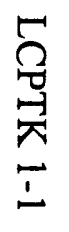 & 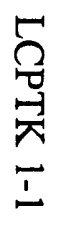 & 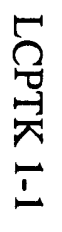 & 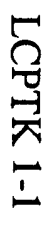 & 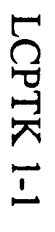 & 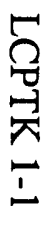 & 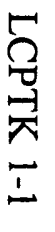 & 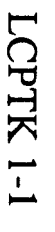 & 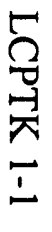 \\
\hline 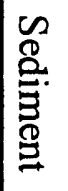 & 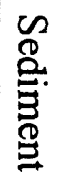 & 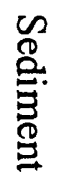 & 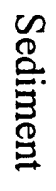 & 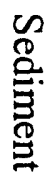 & 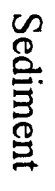 & 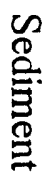 & 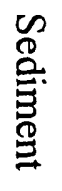 & 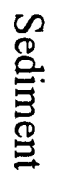 & 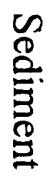 & 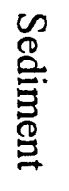 & 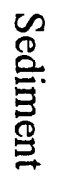 & 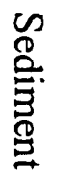 & 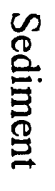 & 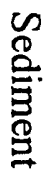 & 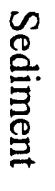 & 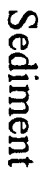 & 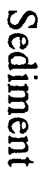 & 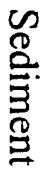 \\
\hline $\begin{array}{l}\overrightarrow{8} \\
0 \\
\frac{1}{2} \\
+\end{array}$ & $\begin{array}{l}\vec{u} \\
\stackrel{+}{\dot{t}} \\
\dot{u}\end{array}$ & $\begin{array}{l}\vec{a} \\
\text { a } \\
\dot{1} \\
\dot{0}\end{array}$ & $\begin{array}{l}\text { 可 } \\
\text { in } \\
\frac{1}{0}\end{array}$ & 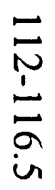 & $\begin{array}{l}\infty \\
0 \\
\infty \\
\infty \\
+\infty\end{array}$ & $\begin{array}{l}\text { o. } \\
1 \\
\text { Na }\end{array}$ & $\begin{array}{l}\stackrel{b}{0} \\
\dot{1} \\
\dot{1}\end{array}$ & $\begin{array}{l}\text { N } \\
\stackrel{\sim}{N}\end{array}$ & $\underset{\infty}{+}$ & 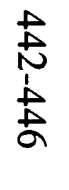 & $\begin{array}{l}\stackrel{\oplus}{\omega} \\
\infty \\
1 \\
\stackrel{+}{N}\end{array}$ & $\begin{array}{l}\stackrel{\oplus}{\omega} \\
\dot{t} \\
\stackrel{+}{\infty}\end{array}$ & $\begin{array}{l}\stackrel{t}{N} \\
\infty \\
\stackrel{1}{\omega} \\
\stackrel{N}{N}\end{array}$ & $\begin{array}{l}\stackrel{A}{N} \\
0 \\
\stackrel{1}{N} \\
\dot{A}\end{array}$ & $\begin{array}{l}\stackrel{\oplus}{\overrightarrow{0}} \\
\stackrel{\leftrightarrow}{ \pm} \\
\vec{A}\end{array}$ & $\begin{array}{l}\omega \\
\infty \\
\infty \\
\omega \\
\omega \\
\text { 心 }\end{array}$ & 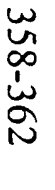 & $\begin{array}{l}\omega \\
\stackrel{\omega}{\omega} \\
\stackrel{\omega}{\omega} \\
\vec{a}\end{array}$ \\
\hline
\end{tabular}




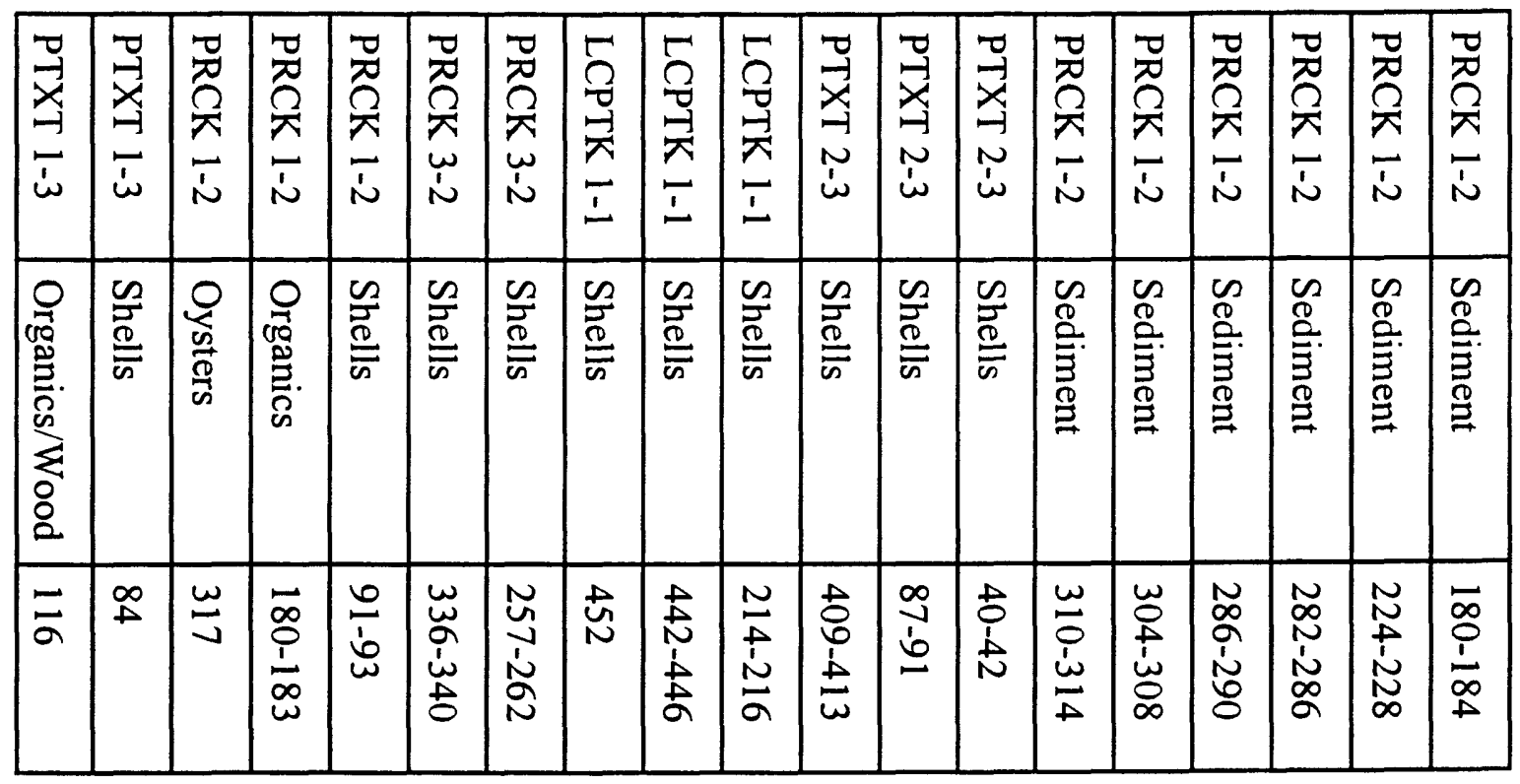

$\infty$

\begin{tabular}{|c|c|c|c|c|c|c|c|c|c|c|c|c|c|c|c|c|c|c|}
\hline 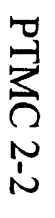 & 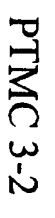 & 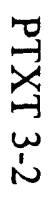 & 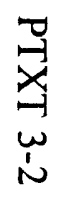 & 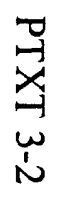 & 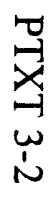 & 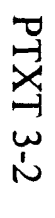 & 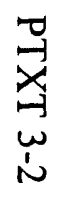 & 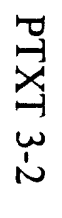 & 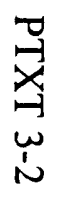 & 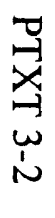 & 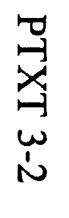 & 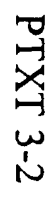 & 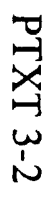 & 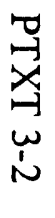 & 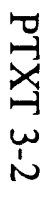 & 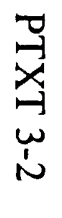 & 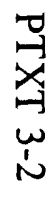 & \\
\hline 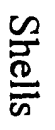 & $\frac{\mathscr{2}}{\bar{\oplus}}$ & $\frac{\sqrt[\infty]{2}}{\overline{\frac{\pi}{n}}}$ & $\frac{\sqrt[\infty]{\sigma}}{\tilde{\sigma}}$ & $\frac{\mathscr{\infty}}{\bar{\sigma}}$ & $\frac{\tilde{\sigma}}{\bar{\sigma}}$ & $\frac{\widetilde{\sigma}}{\bar{\sigma}}$ & $\frac{\frac{\infty}{\sigma}}{\bar{\infty}}$ & $\frac{\mathscr{2}}{\frac{\overrightarrow{0}}{\bar{\infty}}}$ & $\frac{\mathscr{\sigma}}{\bar{\varpi}}$ & $\frac{\mathscr{\Omega}}{\widetilde{\sigma}}$ & $\frac{\mathscr{c}}{\tilde{\sigma}}$ & $\frac{\mathscr{D}}{\bar{\sigma}}$ & $\frac{\mathscr{\sigma}}{\bar{\sigma}}$ & $\frac{\mathscr{\sigma}}{\bar{\phi}}$ & $\frac{\mathscr{\sigma}}{\bar{\sigma}}$ & 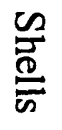 & $\frac{\mathscr{C}}{\tilde{\sigma}}$ & $\frac{\infty}{\sigma}$ \\
\hline $\begin{array}{l}\vec{u} \\
\frac{1}{a} \\
\tilde{\omega}\end{array}$ & $\begin{array}{l}\vec{D} \\
\frac{1}{D} \\
\frac{1}{A}\end{array}$ & $\begin{array}{l}\mathbb{A} \\
\mathbb{O} \\
\stackrel{1}{\mathbb{N}} \\
\mathbb{D}\end{array}$ & $\begin{array}{l}\stackrel{t}{0} \\
\stackrel{1}{t} \\
\stackrel{0}{0}\end{array}$ & $\begin{array}{l}\stackrel{ \pm}{\sim} \\
\stackrel{1}{ \pm} \\
\vec{a}\end{array}$ & $\begin{array}{l}+ \\
0 \\
\infty \\
⺊ \\
\stackrel{1}{N}\end{array}$ & $\begin{array}{l}+ \\
\stackrel{0}{+} \\
+ \\
+ \\
\stackrel{\infty}{\circ}\end{array}$ & $\begin{array}{l}+t \\
8 \\
1 \\
1 \\
0 \\
1\end{array}$ & $\begin{array}{l}w \\
\text { o̊ } \\
1 \\
1 \\
8\end{array}$ & 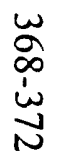 & $\begin{array}{l}\omega \\
\infty \\
\infty \\
\stackrel{\omega}{\omega}\end{array}$ & $\begin{array}{l}\underset{\infty}{\infty} \\
+ \\
\infty \\
\infty \\
\infty\end{array}$ & $\begin{array}{l}\underset{\infty}{\infty} \\
\infty \\
\stackrel{\omega}{W} \\
\infty \\
\perp\end{array}$ & 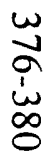 & 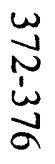 & $\begin{array}{l}\omega \\
\stackrel{\alpha}{+} \\
\dot{t} \\
\stackrel{\alpha}{\Omega}\end{array}$ & $\begin{array}{l}w \\
\text { ô } \\
\dot{\omega} \\
\stackrel{\alpha}{\phi}\end{array}$ & $\begin{array}{l}w \\
\tilde{w} \\
\dot{w} \\
\tilde{\alpha}\end{array}$ & $\begin{array}{l}\bar{N} \\
\stackrel{N}{N} \\
\stackrel{N}{N}\end{array}$ \\
\hline
\end{tabular}




\begin{tabular}{|c|c|c|c|c|c|c|c|c|c|c|c|c|c|c|c|c|c|c|}
\hline 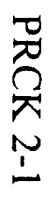 & 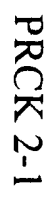 & 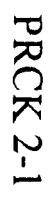 & 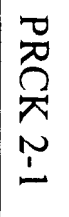 & 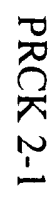 & 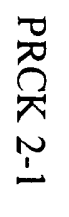 & 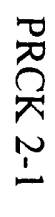 & 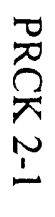 & $\begin{array}{l}\vec{\partial} \\
\underset{-1}{A} \\
\vec{a}\end{array}$ & $\begin{array}{l}5 \\
\vec{\pi} \\
\vec{\pi} \\
\vec{\pi}\end{array}$ & 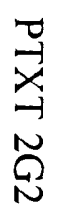 & $\begin{array}{l}\underset{-}{X} \\
\stackrel{-1}{N} \\
\stackrel{N}{N}\end{array}$ & 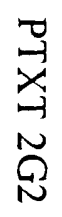 & $\begin{array}{l}\overrightarrow{0} \\
\vec{\Omega} \\
\vec{\Omega}\end{array}$ & 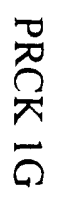 & $\begin{array}{l}\stackrel{\overrightarrow{3}}{3} \\
\stackrel{3}{3} \\
\vec{\Omega}\end{array}$ & 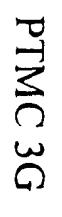 & 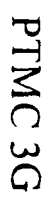 & $\begin{array}{l}\vec{x} \\
\vec{x} \\
\vec{\omega} \\
\dot{\omega}\end{array}$ \\
\hline 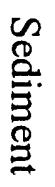 & 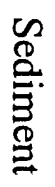 & 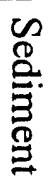 & 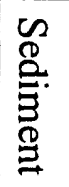 & 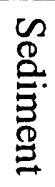 & 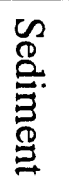 & 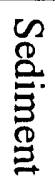 & 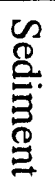 & $\frac{\mathscr{\infty}}{\tilde{\Phi}}$ & $\frac{\mathscr{\infty}}{\stackrel{\Phi}{\infty}}$ & $\frac{\mathscr{\infty}}{\stackrel{\Phi}{\oplus}}$ & $\frac{\mathscr{\infty}}{\frac{\infty}{\infty}}$ & $\frac{\infty}{\frac{\infty}{\sigma}}$ & $\frac{\infty}{\infty}$ & $\frac{\mathscr{\infty}}{\frac{\infty}{\sigma}}$ & $\frac{\mathscr{\infty}}{\frac{\curvearrowright}{\sigma}}$ & $\frac{\infty}{\stackrel{\infty}{હ}}$ & $\frac{\mathscr{2}}{\hbar}$ & $\frac{\mathscr{s}}{\frac{\rho}{\sigma}}$ \\
\hline $\begin{array}{l}\underset{N}{N} \\
\stackrel{N}{N} \\
N\end{array}$ & $\frac{\infty}{\infty} \underset{\infty}{+\infty}$ & $\begin{array}{l}\vec{D} \\
\frac{1}{D} \\
\frac{1}{D}\end{array}$ & $\begin{array}{l}\infty \\
\infty \\
\dot{\omega} \\
\text { N }\end{array}$ & $\begin{array}{c}\infty \\
0 \\
\infty \\
\infty \\
+\infty\end{array}$ & $\begin{array}{l}\stackrel{\vec{N}}{N} \\
\stackrel{1}{a}\end{array}$ & $\begin{array}{l}w \\
\infty \\
1 \\
\stackrel{1}{N}\end{array}$ & $\frac{\infty}{1}$ & 8 & $\begin{array}{l}\text { :্̀ } \\
\text { à }\end{array}$ & 商 & $\begin{array}{l}\text { U. } \\
\text { N }\end{array}$ & $\stackrel{u}{y}$ & $\begin{array}{l}\infty \\
u \\
\infty \\
\sim \\
\sim\end{array}$ & $\ddot{\Delta}$ & ث্ّ & 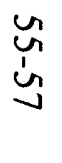 & $\begin{array}{l}\dot{B} \\
\dot{1} \\
\dot{N}\end{array}$ & $\begin{array}{l}\underset{\phi}{\omega} \\
\stackrel{\phi}{\omega}\end{array}$ \\
\hline
\end{tabular}

$\sigma$

\begin{tabular}{|c|c|c|c|c|c|c|c|c|c|c|c|c|c|c|c|c|c|c|}
\hline $\begin{array}{l}\mathscr{\Omega} \\
\mathbb{\infty} \\
\sim \\
\sim \\
\sim\end{array}$ & 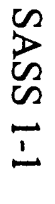 & $\begin{array}{l}\infty \\
\infty \\
\infty \\
\sim \\
\sim \\
-\end{array}$ & $\begin{array}{l}\mathscr{N} \\
\mathbb{2} \\
\sim \\
\vec{D} \\
\vec{D}\end{array}$ & 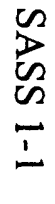 & 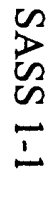 & 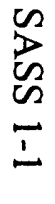 & $\begin{array}{l}\sim \\
D \\
\sim \\
\sim \\
\sim\end{array}$ & $\begin{array}{l}\sim \\
\mathbb{S} \\
\sim \\
\sim \\
\sim \\
\sim\end{array}$ & $\begin{array}{l}\sim \\
\infty \\
\sim \\
\sim \\
\sim \\
-1\end{array}$ & $\begin{array}{l}\sim \\
\Delta \\
\sim \\
\sim \\
\vdots\end{array}$ & $\begin{array}{l}\sim \\
\infty \\
\sim \\
\sim \\
\dot{\sim}\end{array}$ & $\begin{array}{l}\sim \\
\infty \\
\sim \\
\sim \\
\vdots\end{array}$ & $\begin{array}{l}n \\
\text { B } \\
\sim \\
\sim \\
-\end{array}$ & 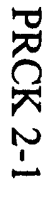 & 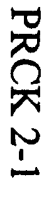 & 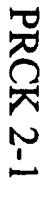 & 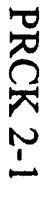 & 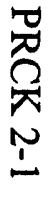 \\
\hline 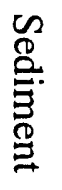 & 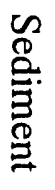 & 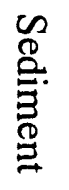 & 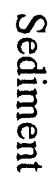 & 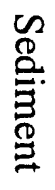 & 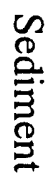 & 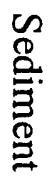 & 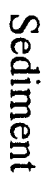 & 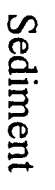 & 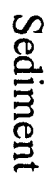 & 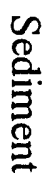 & 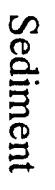 & 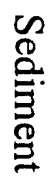 & 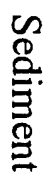 & 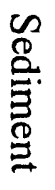 & 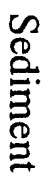 & 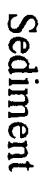 & 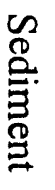 & 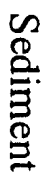 \\
\hline $\begin{array}{l}+ \\
\stackrel{0}{0} \\
\dot{0} \\
\stackrel{\infty}{\circ}\end{array}$ & $\begin{array}{l}\omega \\
\infty \\
o \\
\vdots \\
\omega \\
0\end{array}$ & 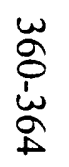 & $\begin{array}{l}\omega \\
\stackrel{\omega}{\omega} \\
\stackrel{\omega}{\omega}\end{array}$ & 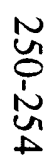 & $\begin{array}{l}N \\
N \\
\infty \\
N \\
N \\
N\end{array}$ & $\begin{array}{l}\frac{N}{\sigma} \\
\stackrel{1}{N} \\
N\end{array}$ & $\begin{array}{l}\overrightarrow{8} \\
\frac{1}{8} \\
\dot{1}\end{array}$ & $\frac{\bar{N}}{\frac{1}{N}}$ & $\begin{array}{l}\text { 응 } \\
\frac{1}{1} \\
\frac{1}{8}\end{array}$ & $\begin{array}{l}\breve{o} \\
\dot{u} \\
\ddot{\phi}\end{array}$ & $\begin{array}{l}\dot{a} \\
\dot{i} \\
\dot{b}\end{array}$ & $\begin{array}{l}\frac{\vec{N}}{a} \\
\stackrel{1}{a}\end{array}$ & $\frac{\overrightarrow{0}}{\Delta}$ & $\begin{array}{l}\stackrel{ \pm}{\overrightarrow{0}} \\
\stackrel{\infty}{1} \\
\stackrel{N}{N}\end{array}$ & 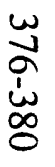 & 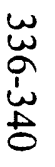 & 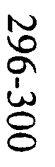 & $\begin{array}{l}\text { U్ } \\
\infty \\
\Lambda \\
\\
\end{array}$ \\
\hline
\end{tabular}




\begin{tabular}{|c|c|c|c|c|c|c|c|c|c|c|c|c|c|c|c|c|c|c|}
\hline $\begin{array}{l}\sim \\
\dot{B} \\
\sim \\
\sim \\
N \\
\tilde{N}\end{array}$ & $\begin{array}{l}n \\
D \\
L \\
\sim \\
N \\
N \\
N\end{array}$ & $\begin{array}{l}\sim \\
D \\
\sim \\
\sim \\
N \\
N\end{array}$ & $\begin{array}{l}\infty \\
D \\
\infty \\
\sim \\
N \\
N\end{array}$ & $\begin{array}{l}\infty \\
\infty \\
\infty \\
\sim \\
N\end{array}$ & 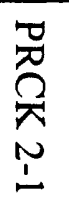 & 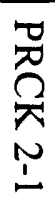 & $\begin{array}{l}\infty \\
B \\
\sim \\
\sim \\
N \\
N\end{array}$ & $\begin{array}{l}\sim \\
D \\
\sim \\
\sim \\
N \\
\tilde{N}\end{array}$ & $\begin{array}{l}\infty \\
\dot{\infty} \\
\sim \\
\sim \\
N\end{array}$ & $\begin{array}{l}\infty \\
D \\
\sim \\
\sim \\
N \\
\text { N }\end{array}$ & $\begin{array}{l}\infty \\
D \\
\infty \\
\sim \\
N \\
N\end{array}$ & $\begin{array}{l}\infty \\
\sim \\
\sim \\
N \\
N \\
N\end{array}$ & $\begin{array}{l}\infty \\
\mathbb{B} \\
\sim \\
N \\
\dot{N}\end{array}$ & $\begin{array}{l}\infty \\
\infty \\
\infty \\
N \\
N\end{array}$ & $\begin{array}{l}\infty \\
D \\
\infty \\
\sim \\
N \\
N\end{array}$ & $\begin{array}{l}\infty \\
\infty \\
\infty \\
\sim \\
N \\
N\end{array}$ & $\begin{array}{l}\infty \\
D \\
\infty \\
\sim \\
N \\
N\end{array}$ & $\begin{array}{l}\sim \\
\sim \\
\sim \\
\sim \\
N \\
N\end{array}$ \\
\hline 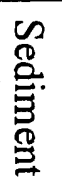 & 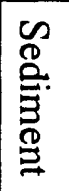 & $\begin{array}{l}\infty \\
\mathbb{2} \\
\stackrel{2}{3} \\
\stackrel{2}{0}\end{array}$ & 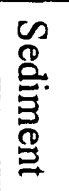 & 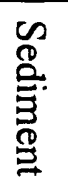 & $\stackrel{\infty}{\stackrel{\infty}{\oplus}}$ & $\stackrel{\infty}{\stackrel{\omega}{\sigma}}$ & 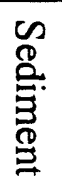 & 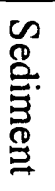 & 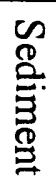 & 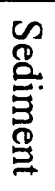 & 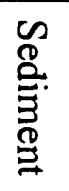 & 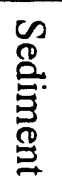 & 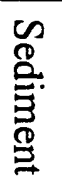 & 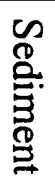 & 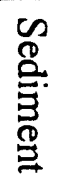 & 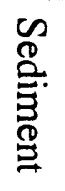 & 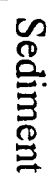 & 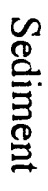 \\
\hline 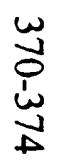 & $\begin{array}{l}\underset{t}{\omega} \\
\underset{+}{\omega} \\
\underset{\infty}{\omega}\end{array}$ & $\begin{array}{l}\underset{\omega}{\omega} \\
\text { 它 } \\
\underset{\omega}{\omega}\end{array}$ & $\begin{array}{l}\omega \\
\omega \\
0 \\
\omega \\
\dot{0}\end{array}$ & $\underset{\infty}{\omega} \underset{\omega}{\omega}$ & $\begin{array}{c}\infty \\
+ \\
\infty \\
\infty\end{array}$ & $\begin{array}{l}\stackrel{9}{+} \\
\stackrel{+}{\infty}\end{array}$ & $\begin{array}{l}\tilde{N} \\
\stackrel{N}{*} \\
\tilde{N} \\
\infty\end{array}$ & 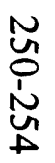 & 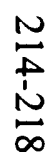 & $\begin{array}{l}\vec{b} \\
\frac{1}{2} \\
\stackrel{0}{0}\end{array}$ & $\begin{array}{l}\vec{D} \\
\stackrel{D}{D}\end{array}$ & $\begin{array}{l}\vec{\circ} \\
\frac{1}{\circ} \\
\stackrel{\phi}{+}\end{array}$ & 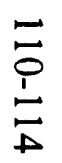 & $\begin{array}{l}\infty \\
\infty \\
\infty \\
+\infty\end{array}$ & 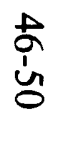 & $\begin{array}{l}+ \\
\dot{0} \\
\dot{1}\end{array}$ & $\begin{array}{l}\text { No } \\
\stackrel{\omega}{\omega}\end{array}$ & $\begin{array}{l}\vec{\infty} \\
\stackrel{1}{ } \\
\stackrel{1}{*}\end{array}$ \\
\hline
\end{tabular}

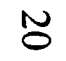

\begin{tabular}{|c|c|c|c|c|c|c|c|c|c|c|c|c|c|c|c|c|c|}
\hline 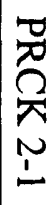 & 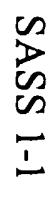 & $\begin{array}{l}\sim \\
D \\
\sim \\
\sim \\
\pm \\
\pm\end{array}$ & $\begin{array}{l}\sim \\
\mathbb{D} \\
\sim \\
\sim \\
\stackrel{1}{1}\end{array}$ & $\begin{array}{l}\infty \\
\tilde{D} \\
\tilde{\omega} \\
\dot{1}\end{array}$ & $\begin{array}{l}\infty \\
\mathbb{B} \\
\sim \\
\sim \\
亡\end{array}$ & 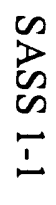 & م & 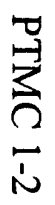 & $\begin{array}{l}\infty \\
\infty \\
\infty \\
N \\
N\end{array}$ & $\begin{array}{l}\infty \\
\mathbb{B} \\
\sim \\
N \\
N\end{array}$ & $\begin{array}{l}\sim \\
\mathbb{B} \\
\sim \\
N \\
N\end{array}$ & $\begin{array}{l}\infty \\
\sim \\
\sim \\
\sim \\
\vdots\end{array}$ & $\begin{array}{l}\infty \\
\sim \\
\infty \\
\sim \\
\varpi\end{array}$ & 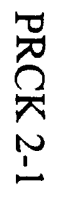 & 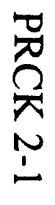 & $\begin{array}{l}\infty \\
\text { D } \\
\infty \\
N \\
N\end{array}$ & 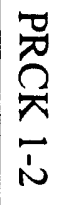 \\
\hline 疋 & 莕. & 菅. & 忐. & 㤀 & 㞼. & 予 & $\stackrel{\stackrel{\infty}{\overparen{C}}}{\stackrel{\Xi}{\Xi}}$ & $\stackrel{\stackrel{\infty}{\Phi}}{\stackrel{\oplus}{=}}$ & $\underset{8}{8}$ & 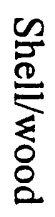 & 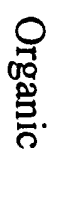 & 交. & 疋. & 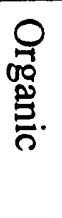 & $\stackrel{\mathscr{\Phi}}{\stackrel{\mathscr{\Phi}}{=}}$ & $\begin{array}{l}\stackrel{\mathscr{S}}{\Phi} \\
\stackrel{\oplus}{=}\end{array}$ & 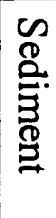 \\
\hline 衣 & $\begin{array}{l}+ \\
8 \\
\dot{8} \\
\dot{8} \\
\stackrel{8}{8}\end{array}$ & 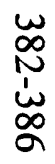 & $\begin{array}{l}w \\
\text { on } \\
\dot{\omega} \\
\text { ğ }\end{array}$ & 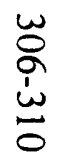 & $\begin{array}{l}\text { N } \\
\text { ô } \\
\text { N } \\
\text { U. }\end{array}$ & $\frac{N}{\stackrel{N}{N}}$ & 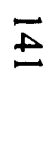 & $\bar{a}$ & $\begin{array}{l}\underset{+}{ \pm} \\
\dot{\omega} \\
\stackrel{\Delta}{N}\end{array}$ & 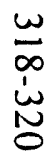 & $\begin{array}{l}\bar{\omega} \\
\stackrel{1}{0} \\
\underline{\omega}\end{array}$ & $\begin{array}{l}\bar{u} \\
\stackrel{1}{ } \\
\dot{g}\end{array}$ & $\begin{array}{l}\overline{\bar{D}} \\
\dot{1} \\
\bar{N}\end{array}$ & $\begin{array}{l}\text { No } \\
\stackrel{0}{\sim} \\
\text { No }\end{array}$ & $\frac{9}{\dot{\phi}}$ & $\frac{\vec{N}}{\frac{N}{N}}$ & $\begin{array}{l}\bar{n} \\
\infty \\
\frac{1}{\sigma} \\
\sigma\end{array}$ \\
\hline
\end{tabular}




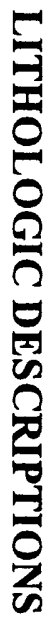




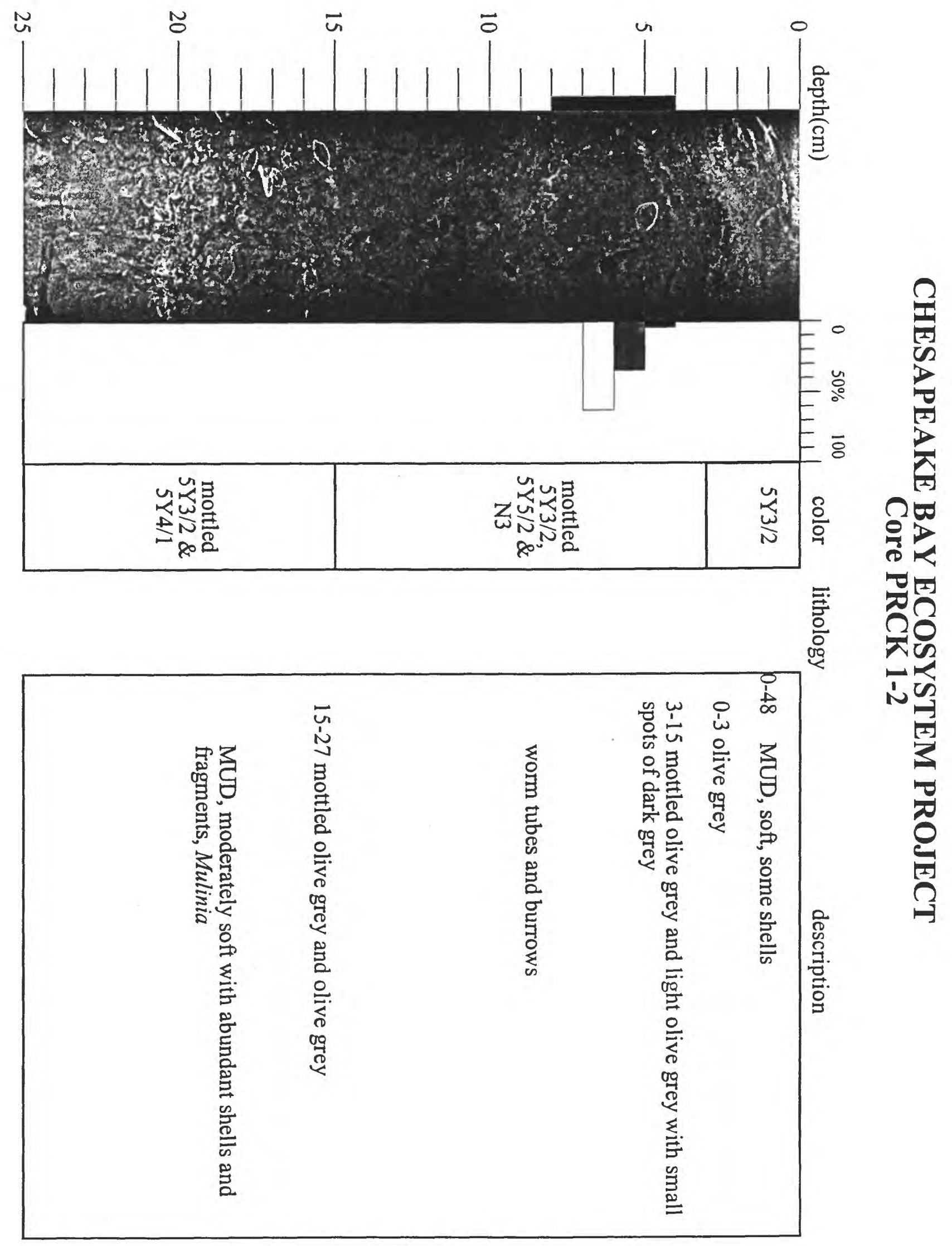



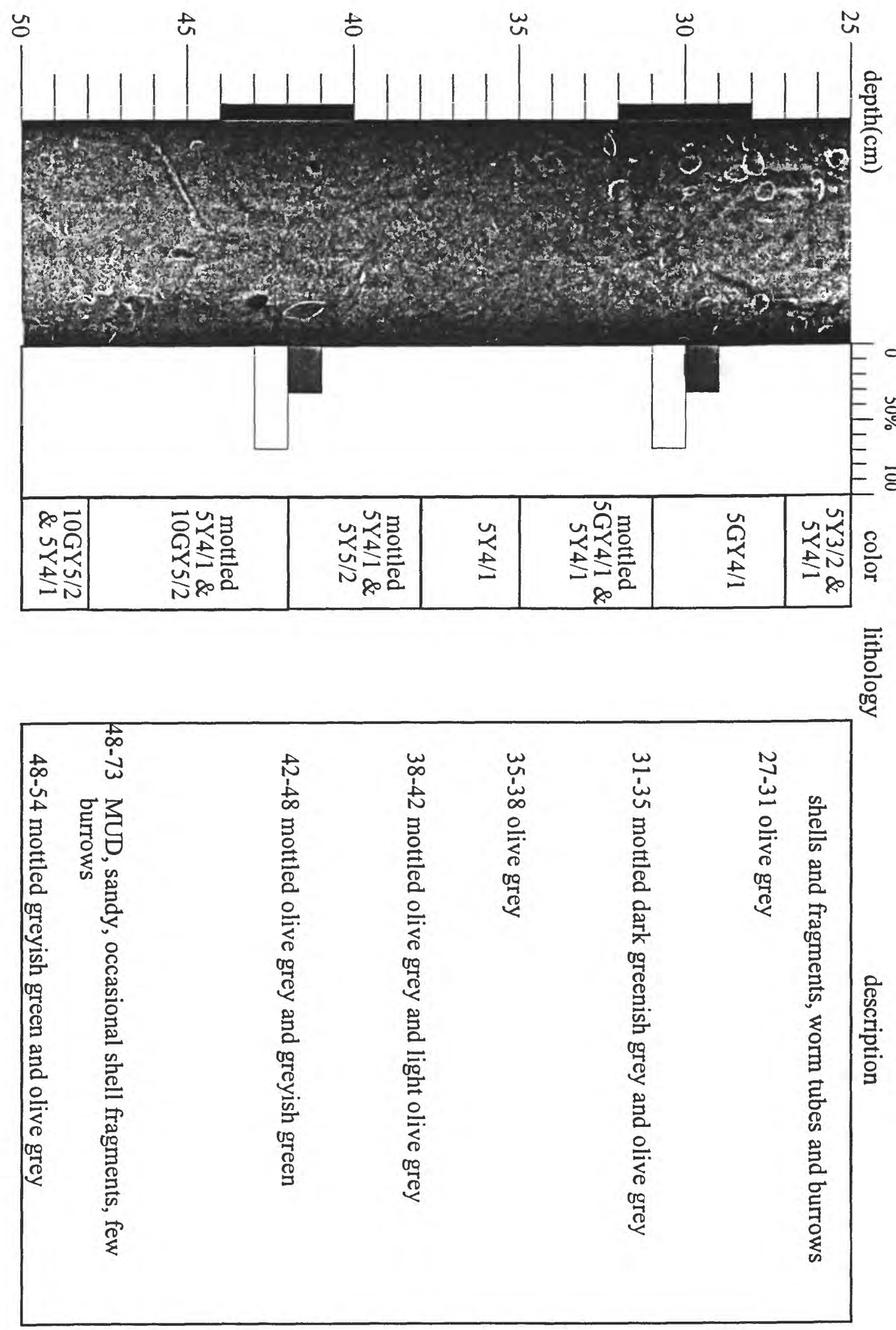

壱 

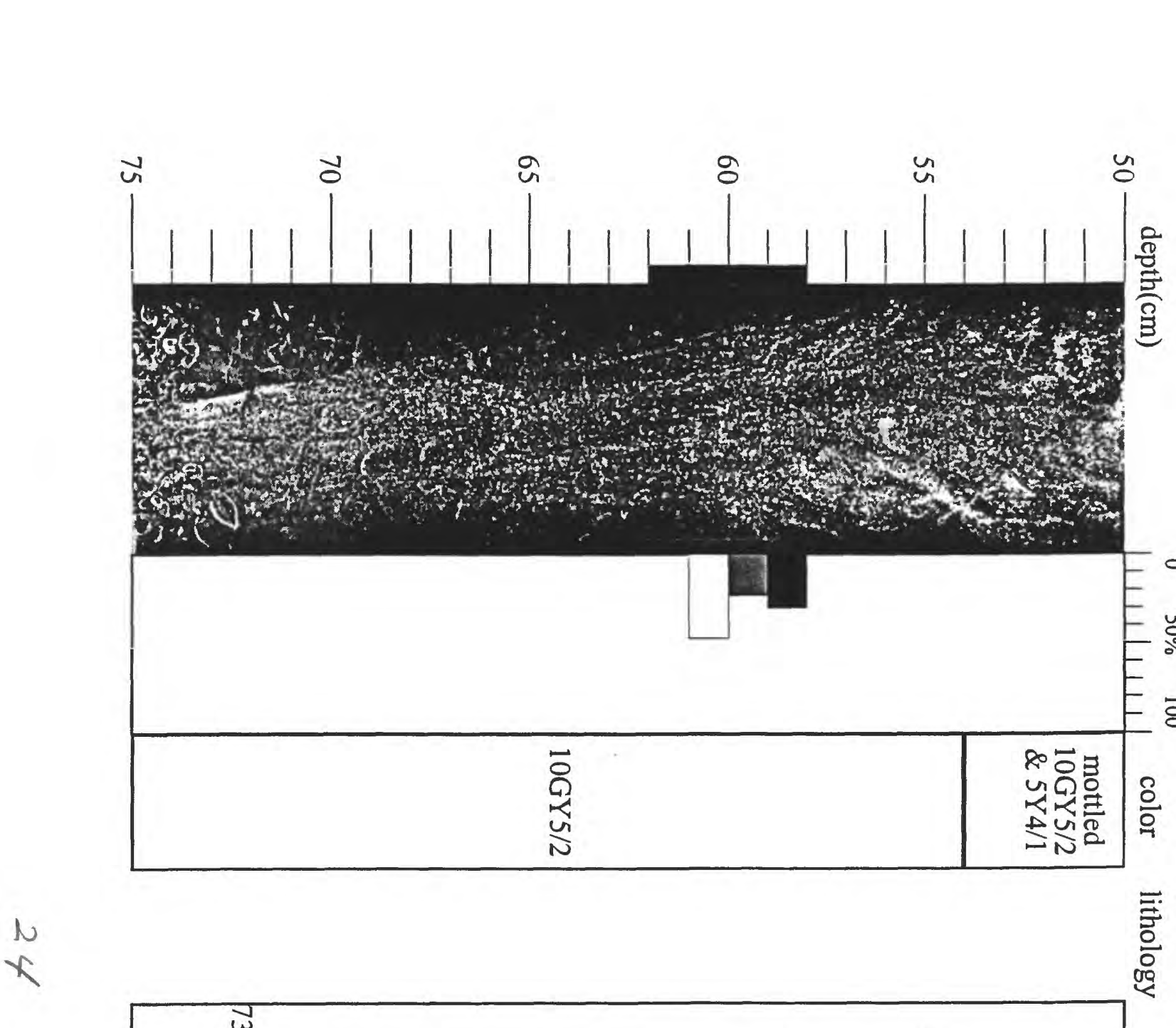

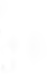
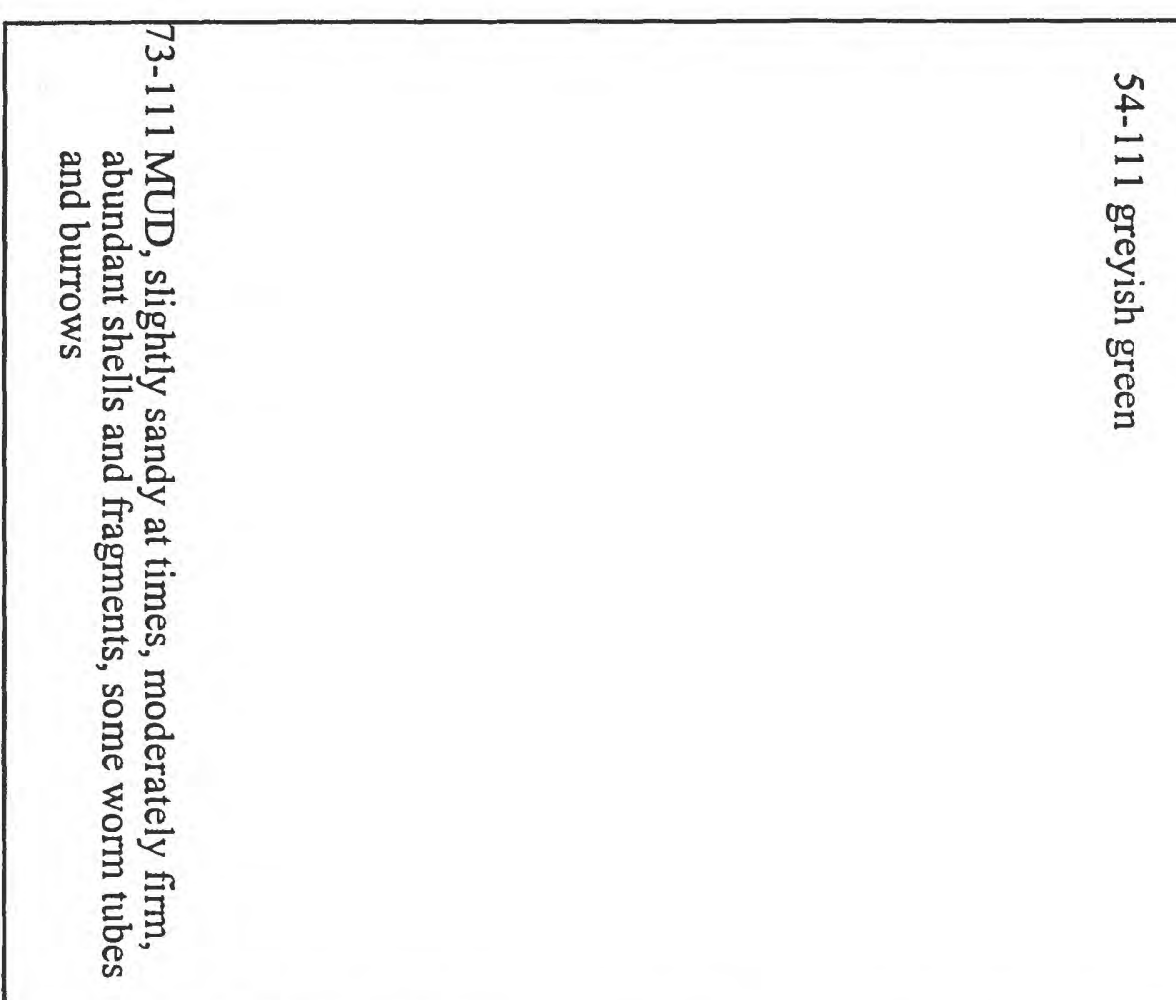


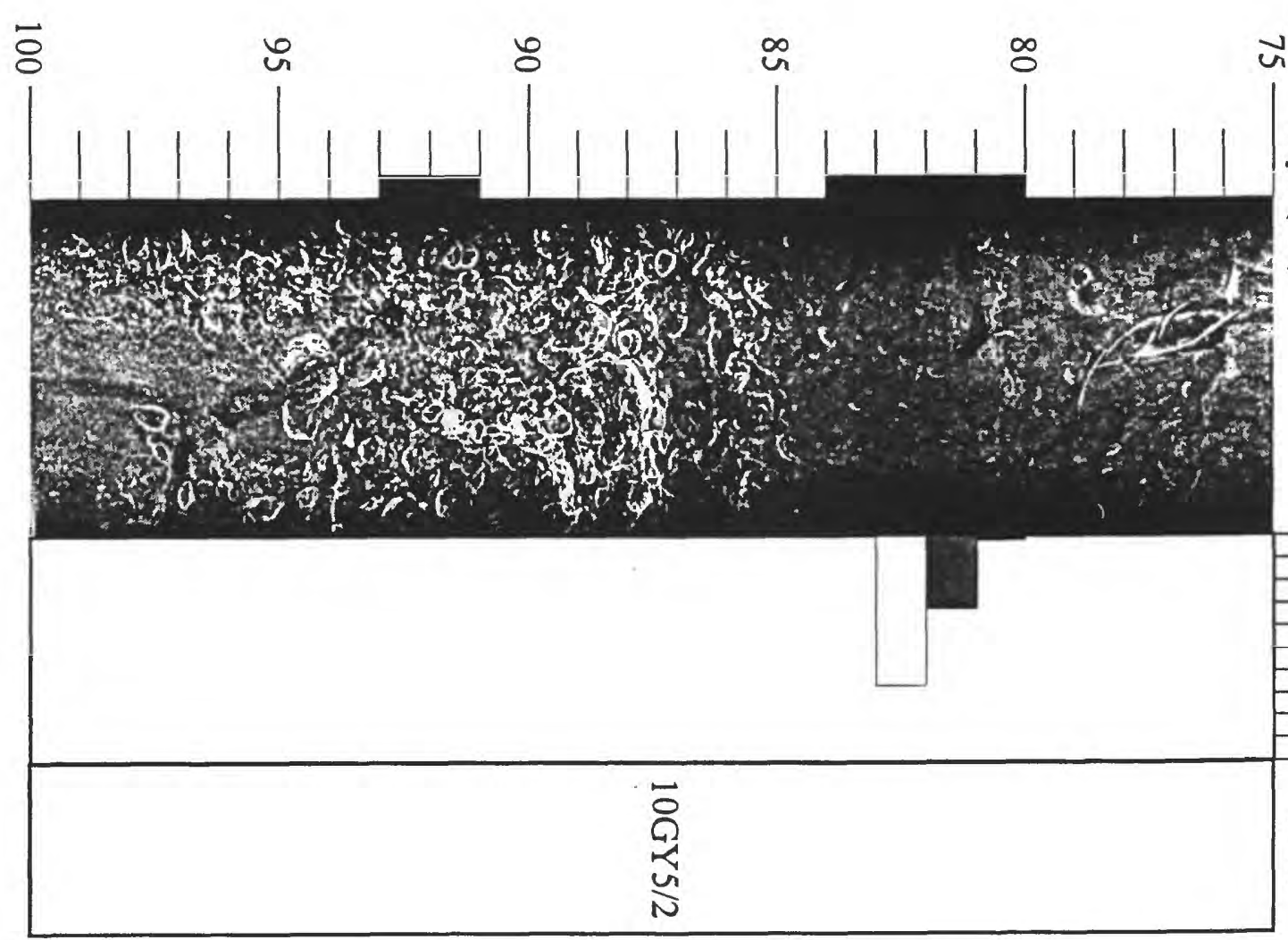

v

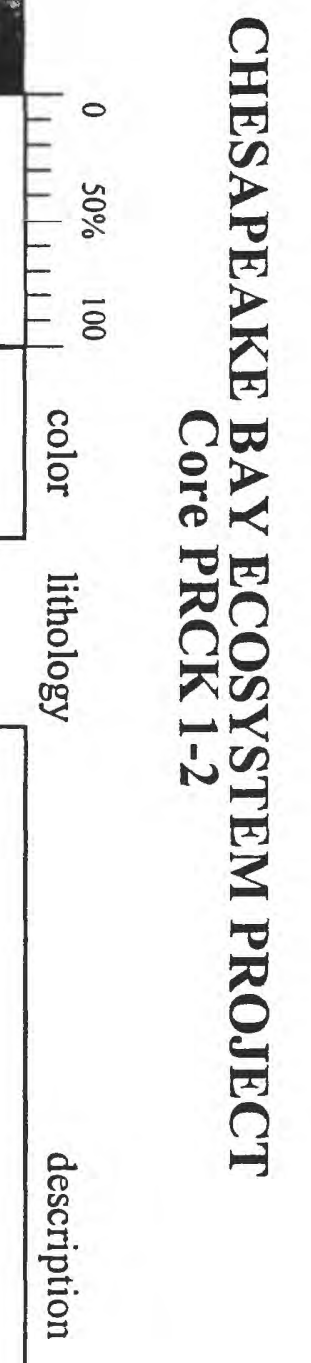



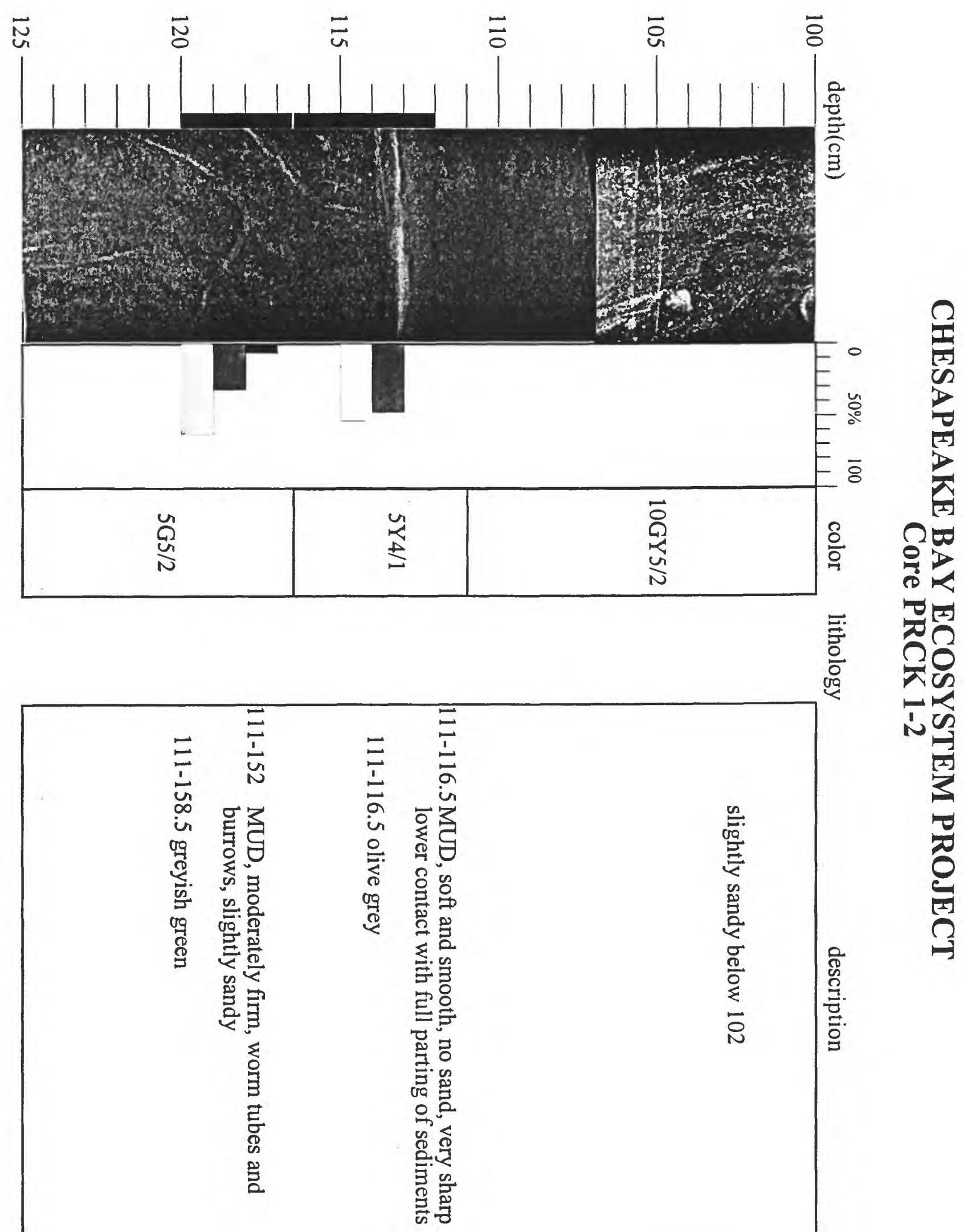


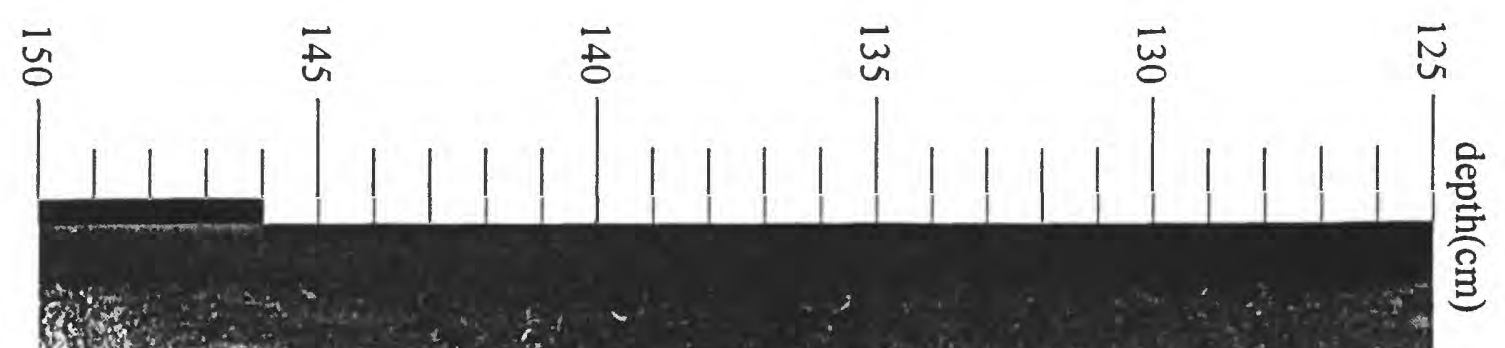

$v$
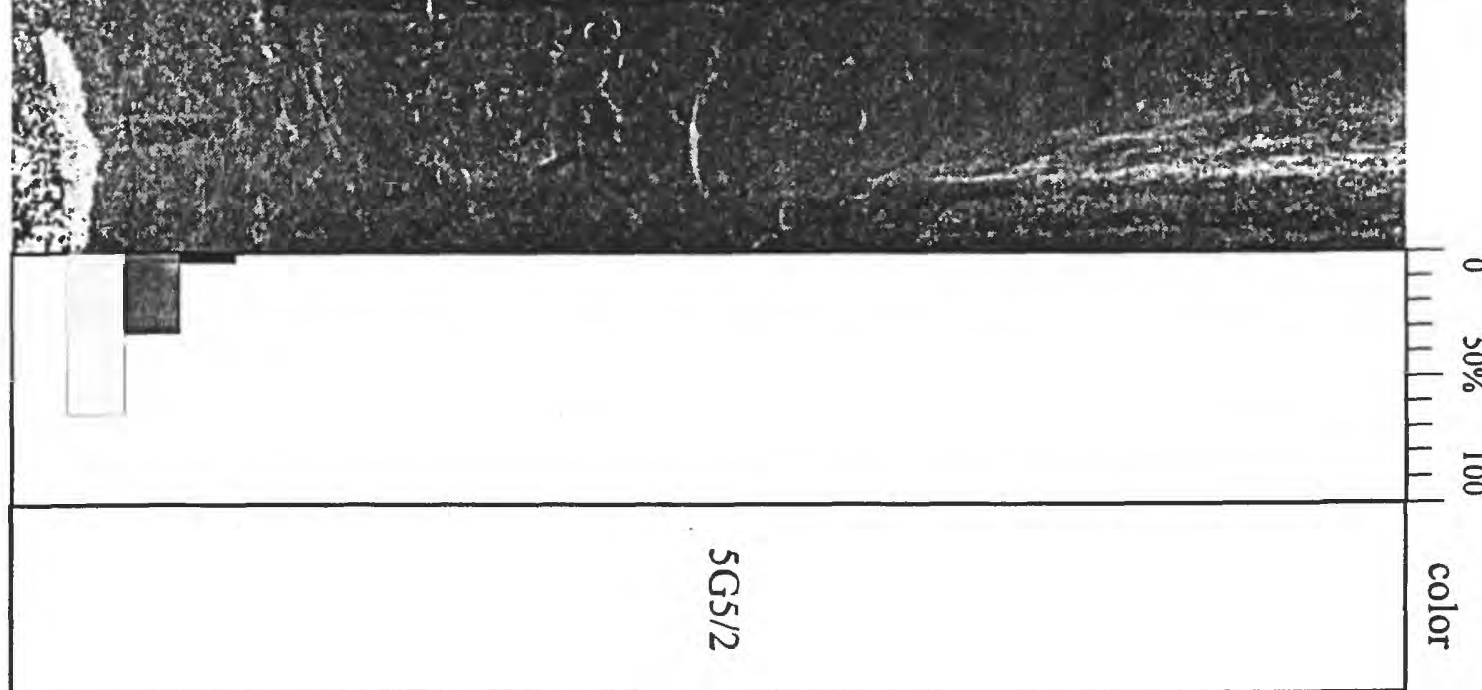

D

$\stackrel{n}{n}$

퐁

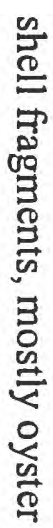

吉哇

居

莺

1

:

逭

8

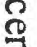

蛋 


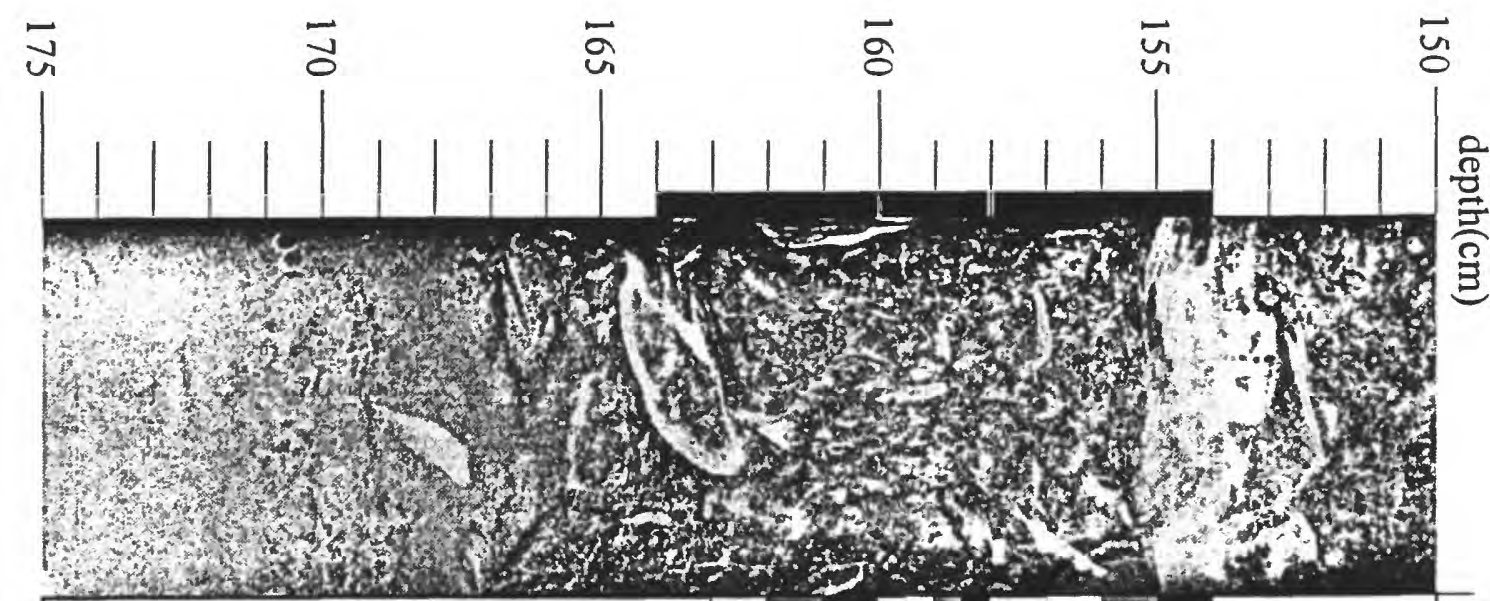

$N$

\begin{tabular}{|c|c|c|c|c|}
\hline 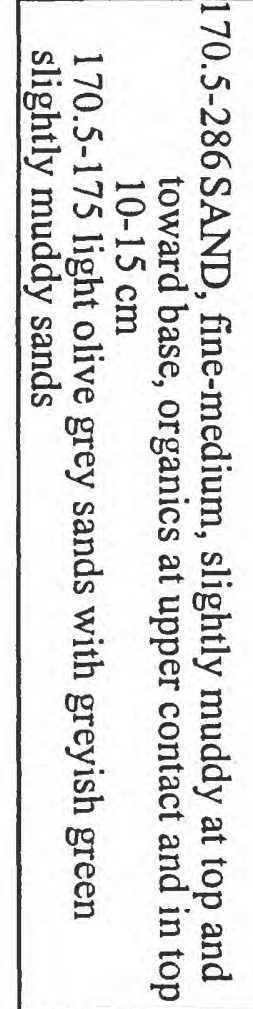 & 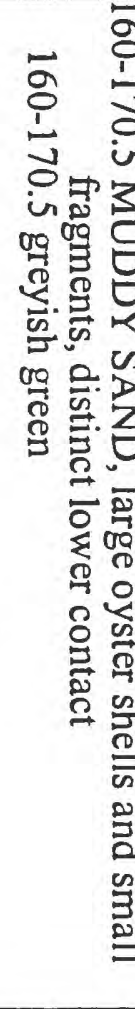 & 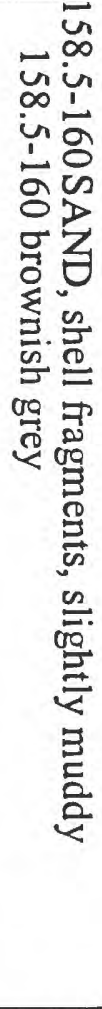 & 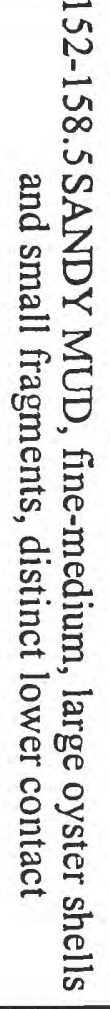 & \\
\hline
\end{tabular}



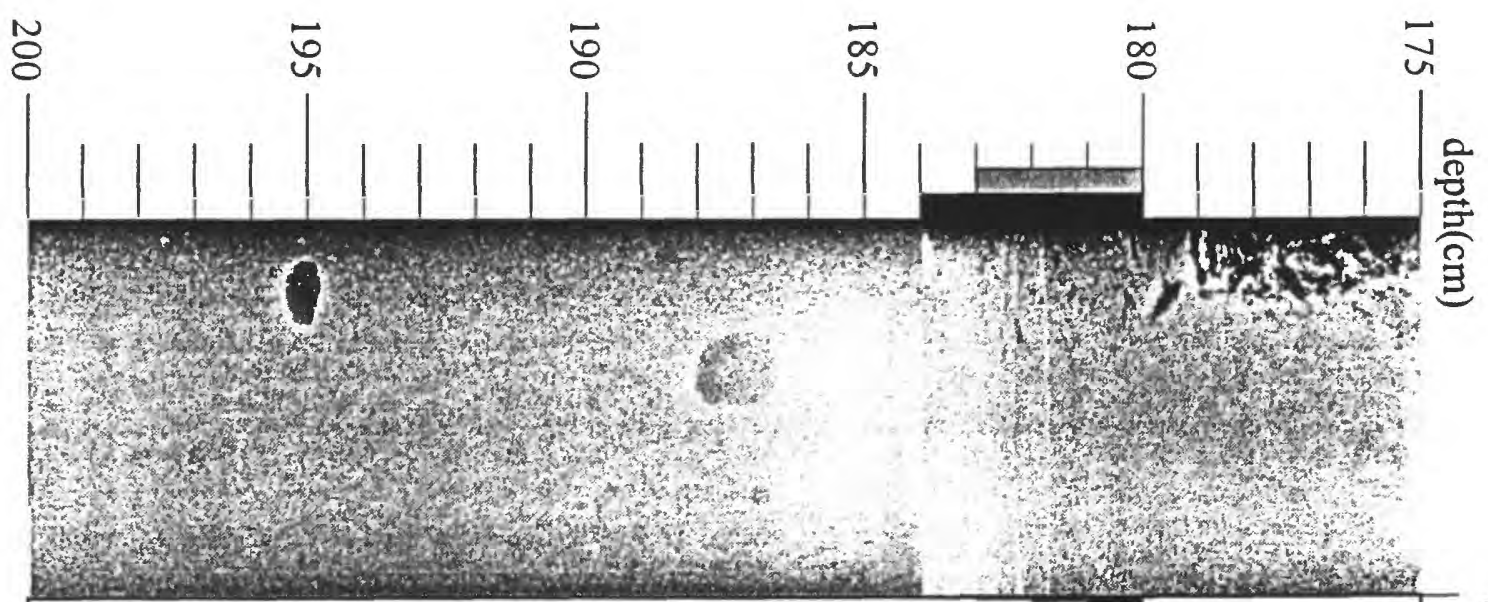

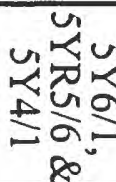

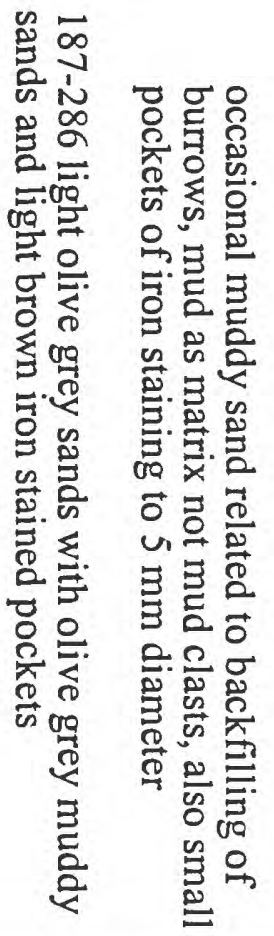

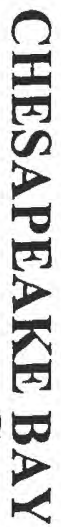

言

U⿱宀

? 

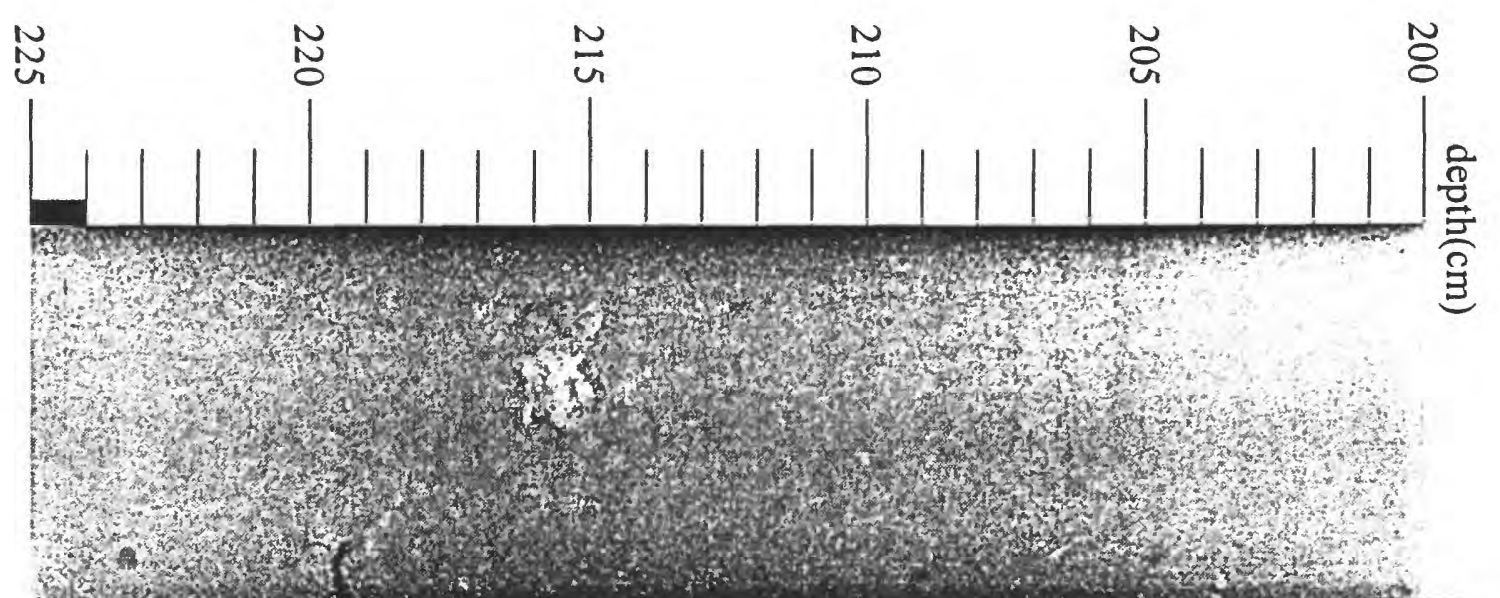

$=$ -

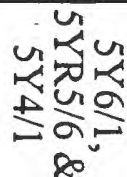

豆

气. 


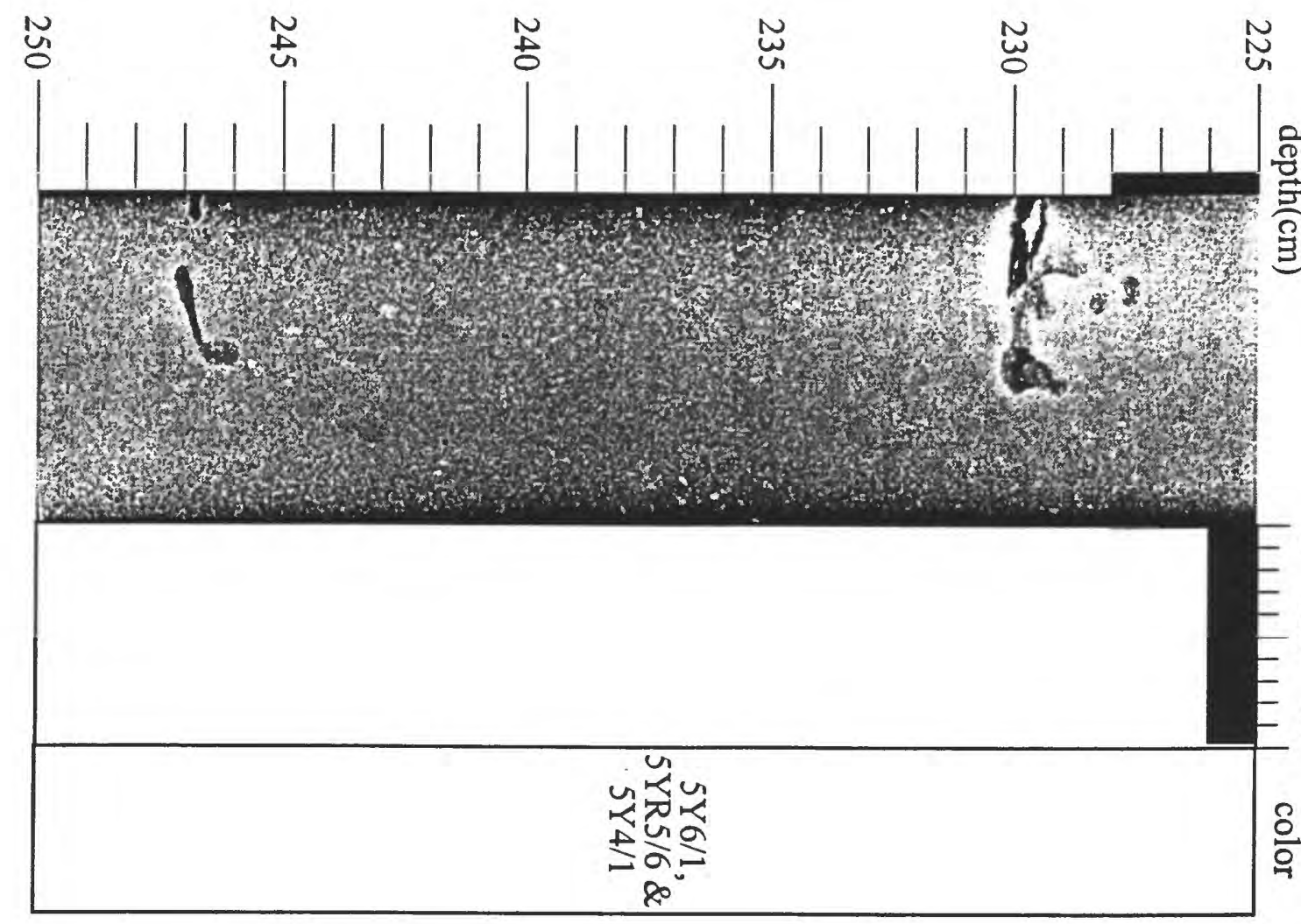

$\omega$

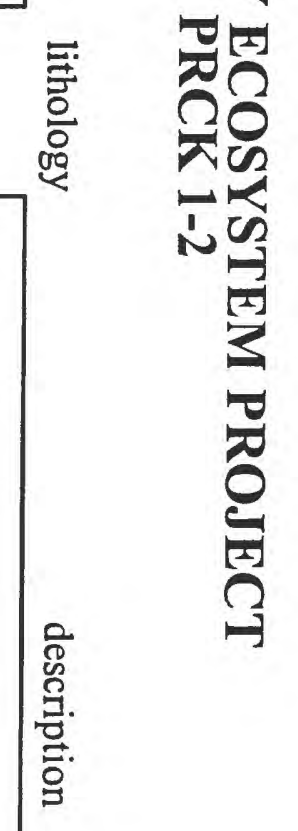



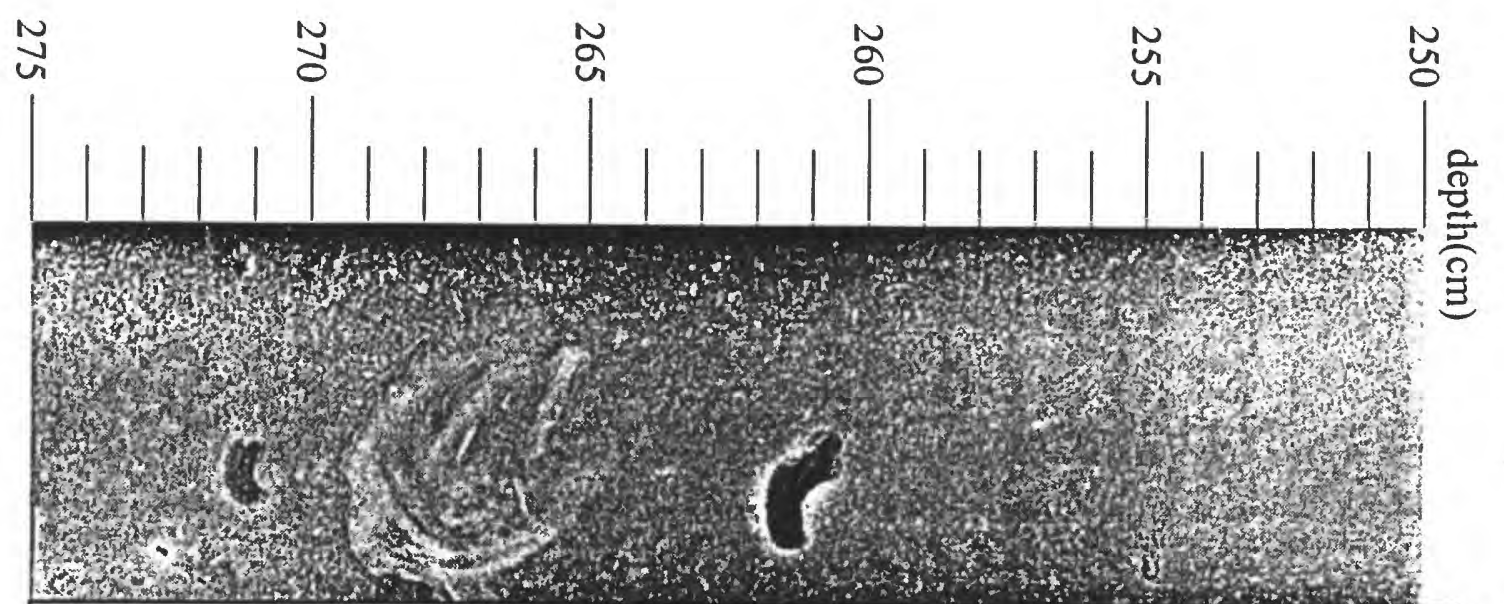


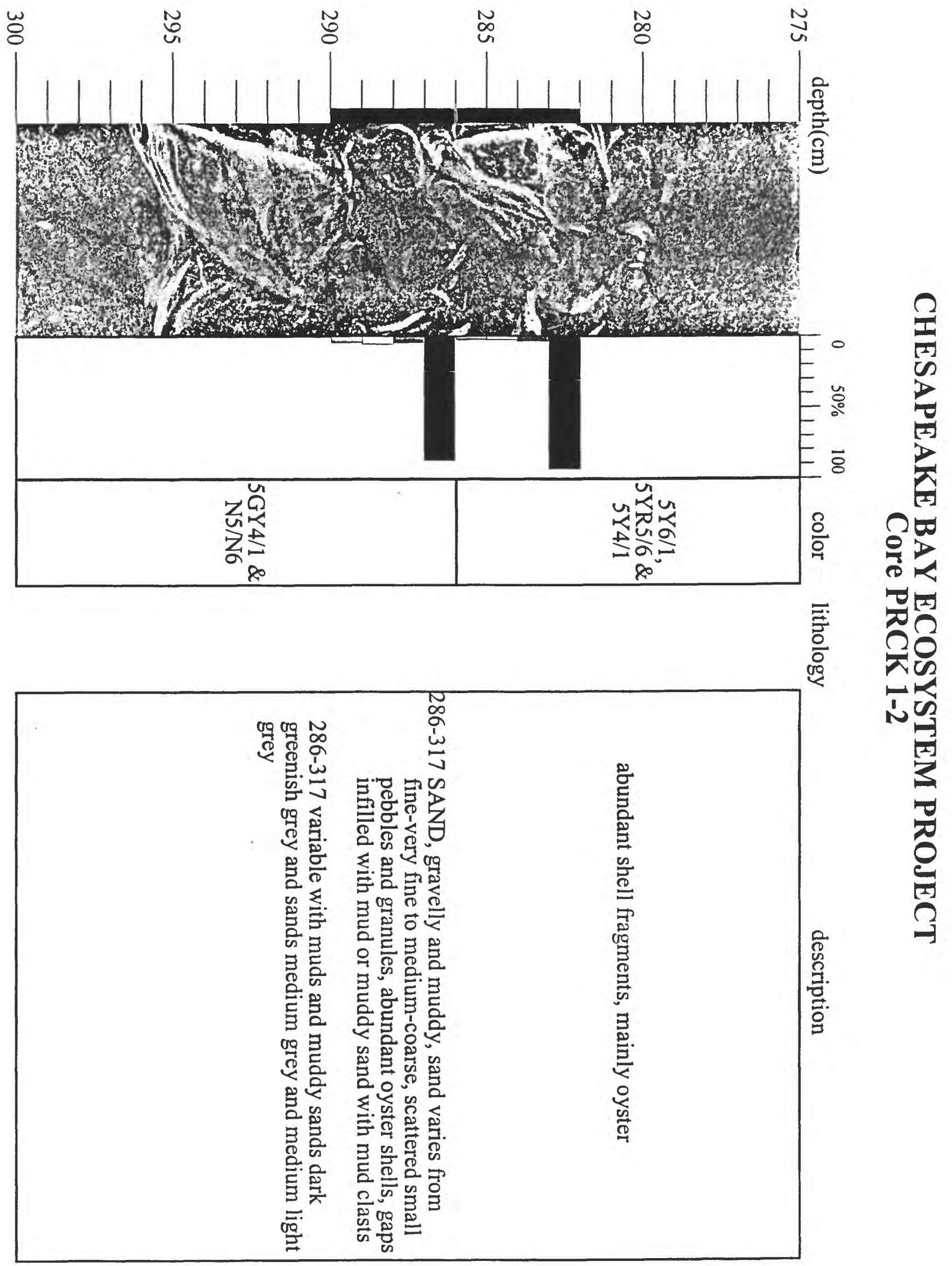




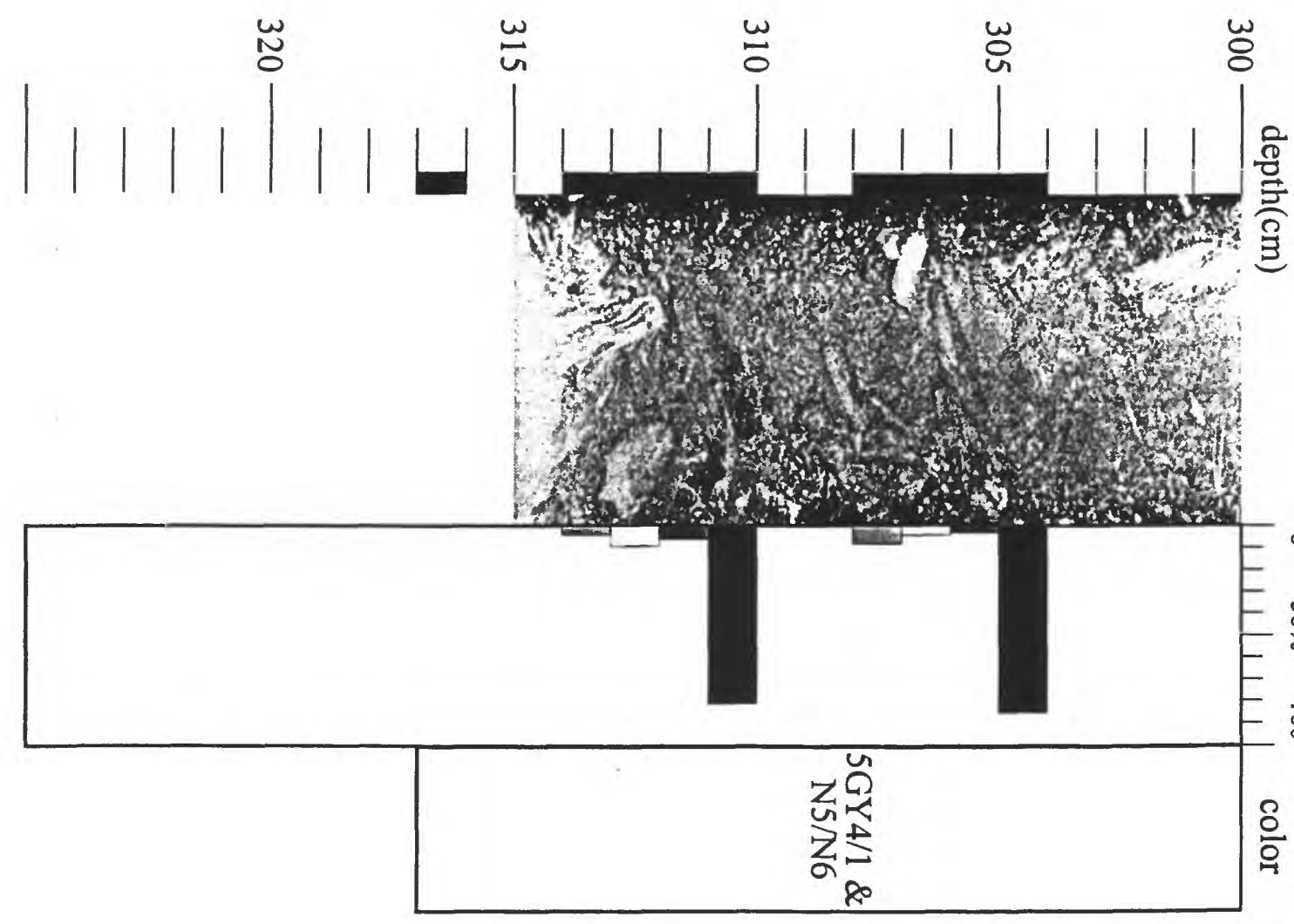

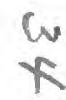

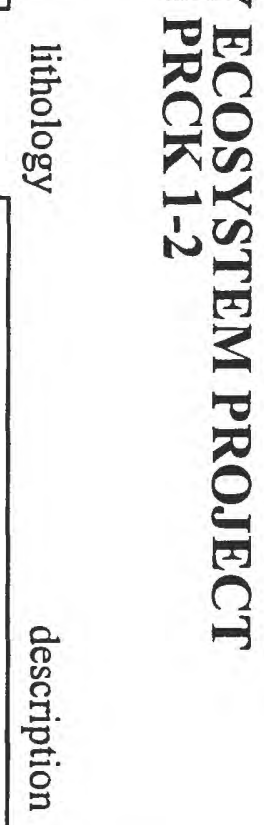




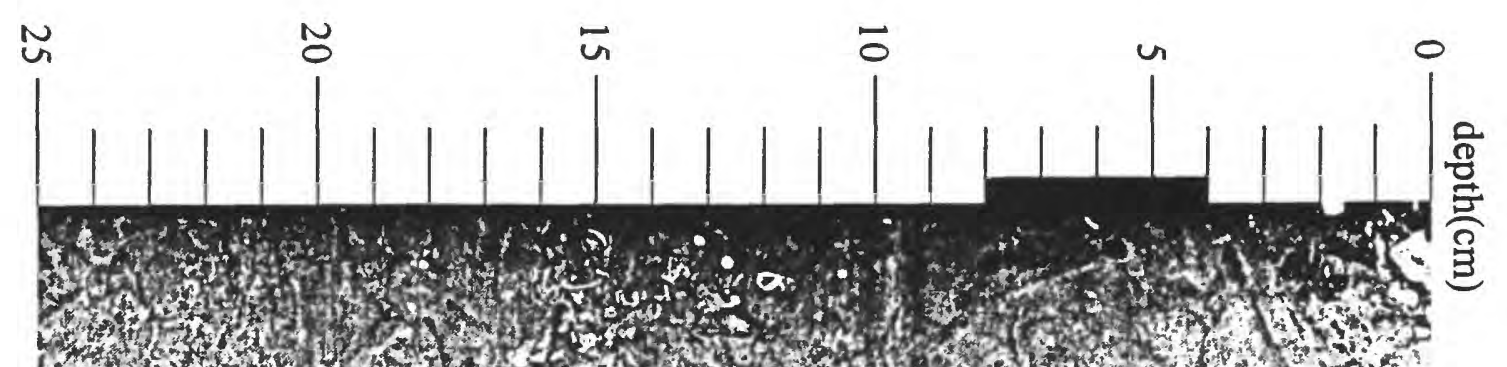

$w$
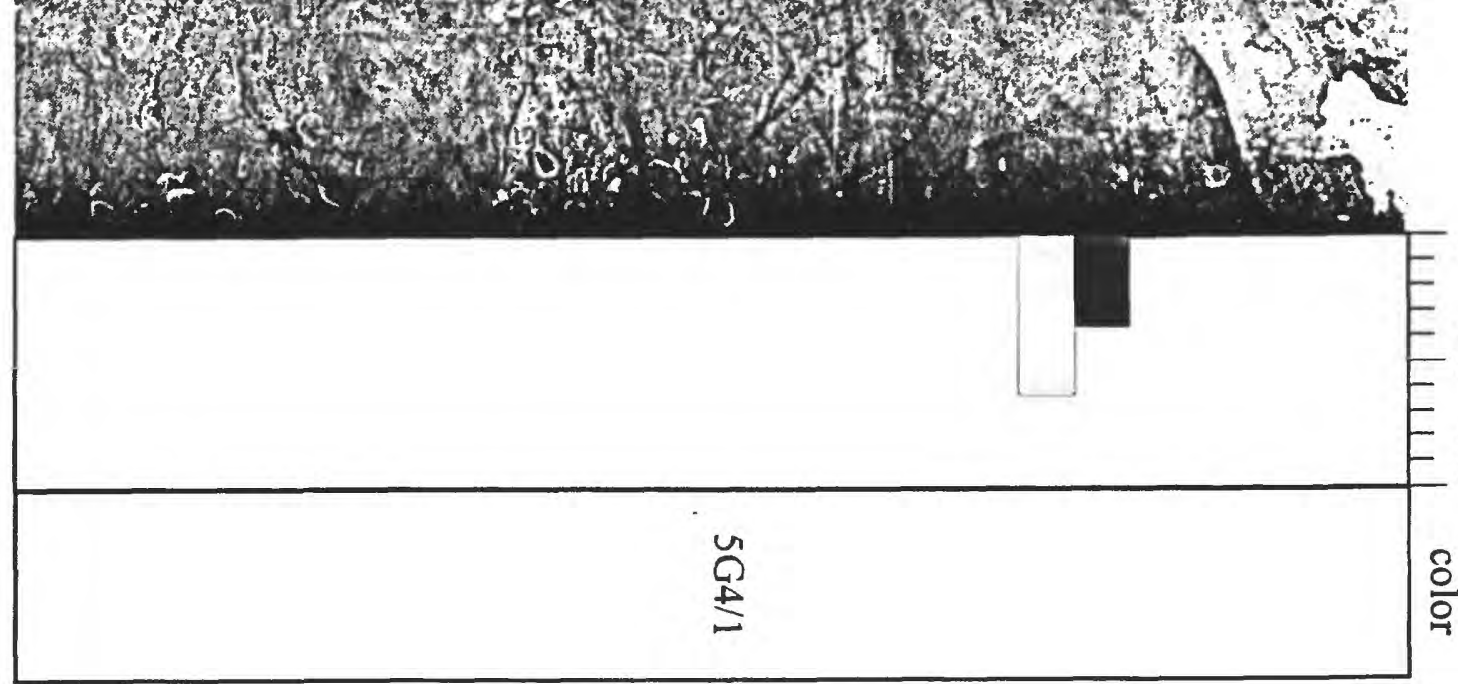

E

,

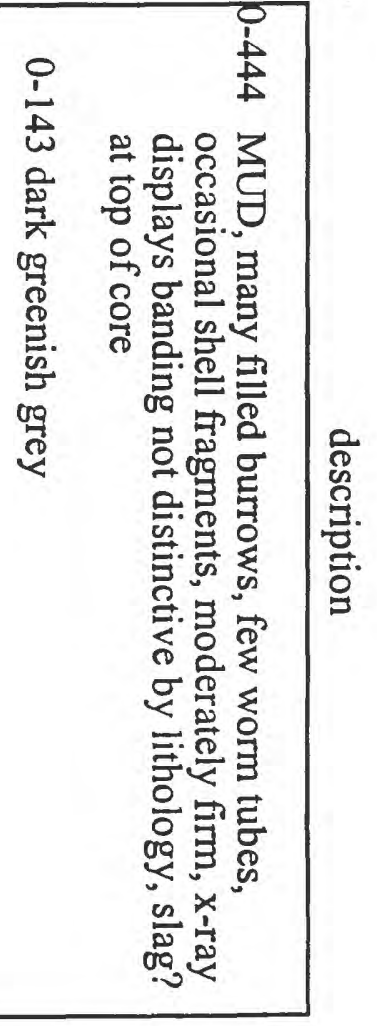




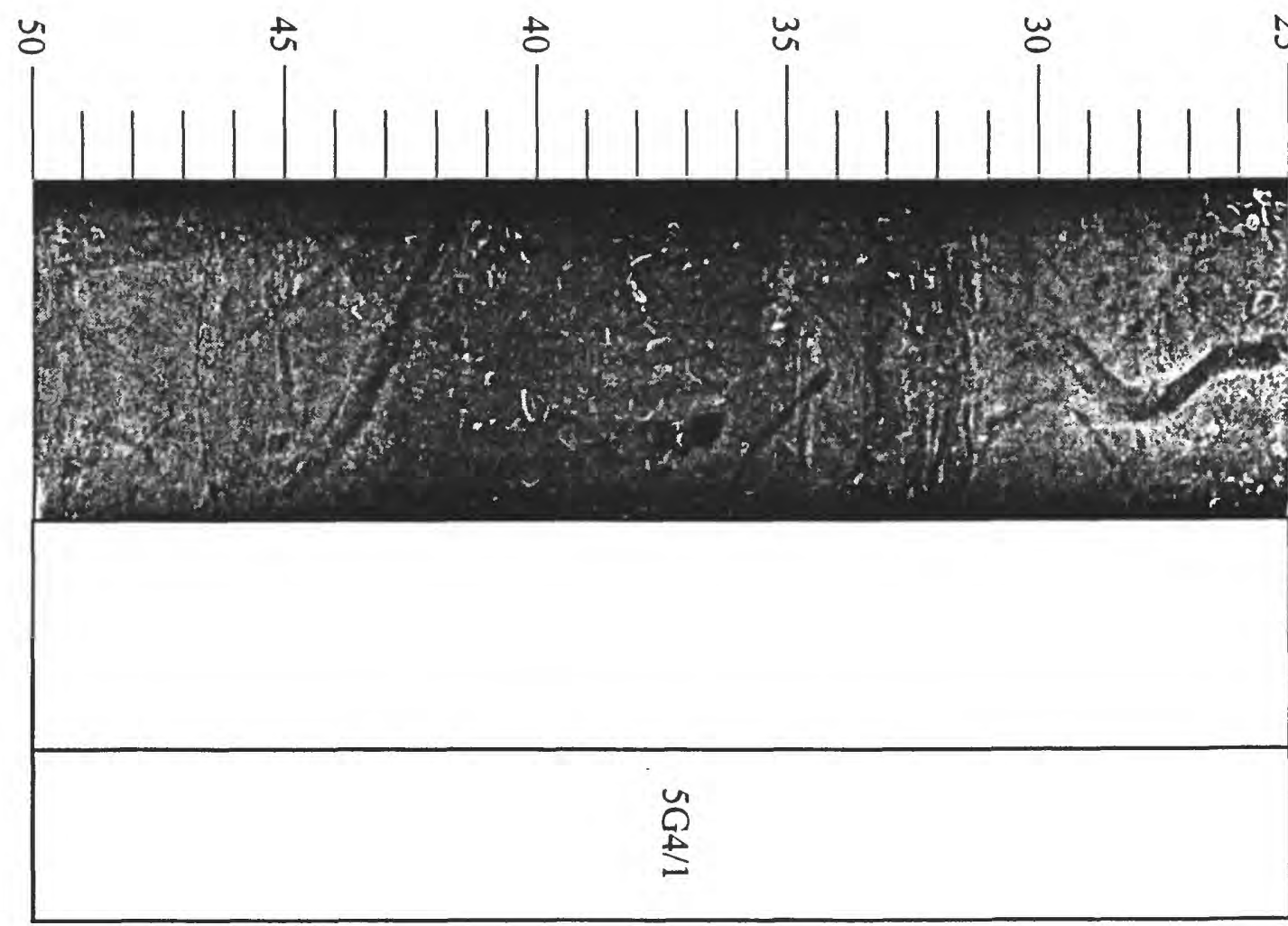

$\omega$

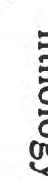

E。

言

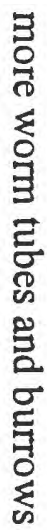

号. 


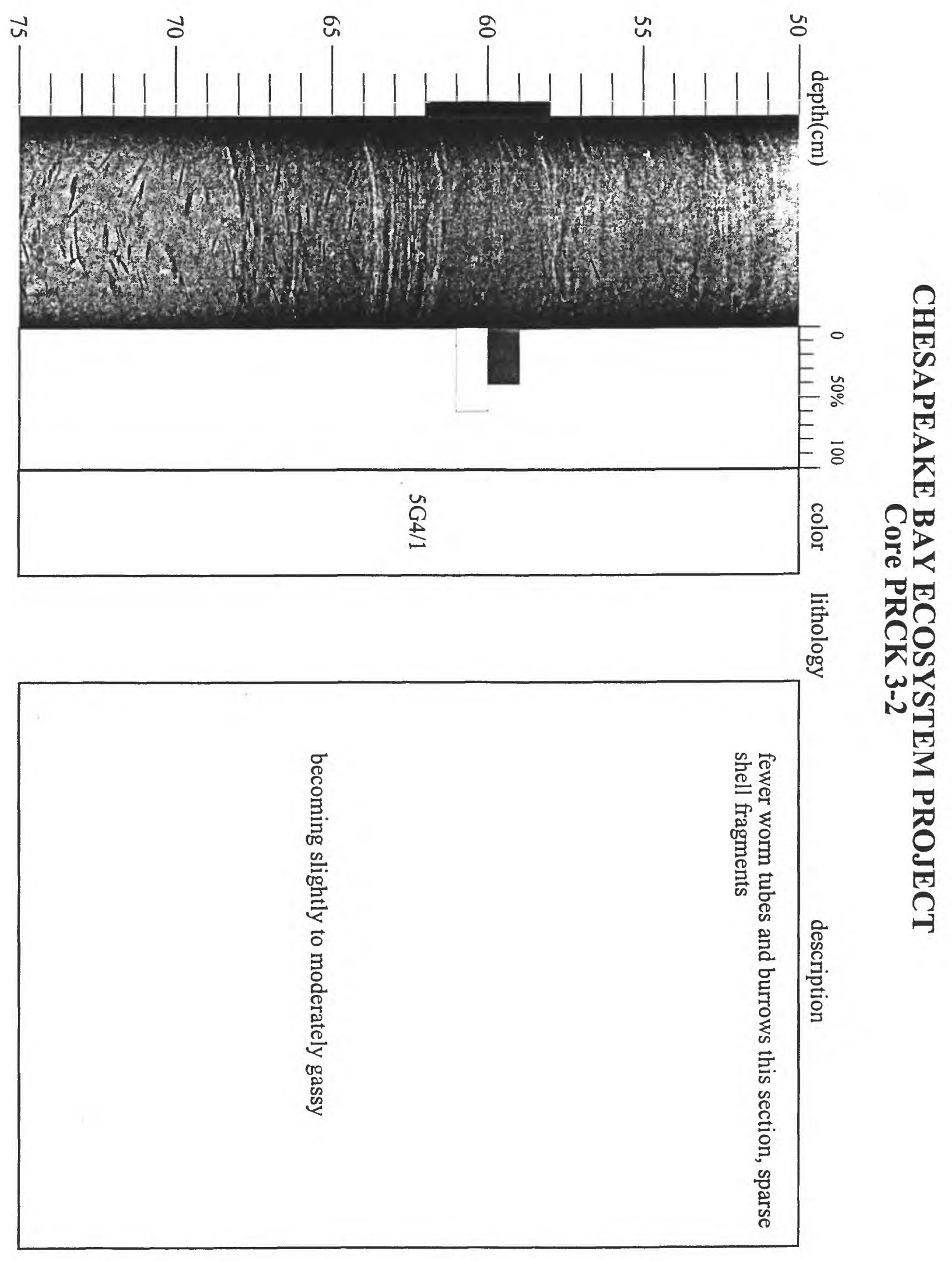




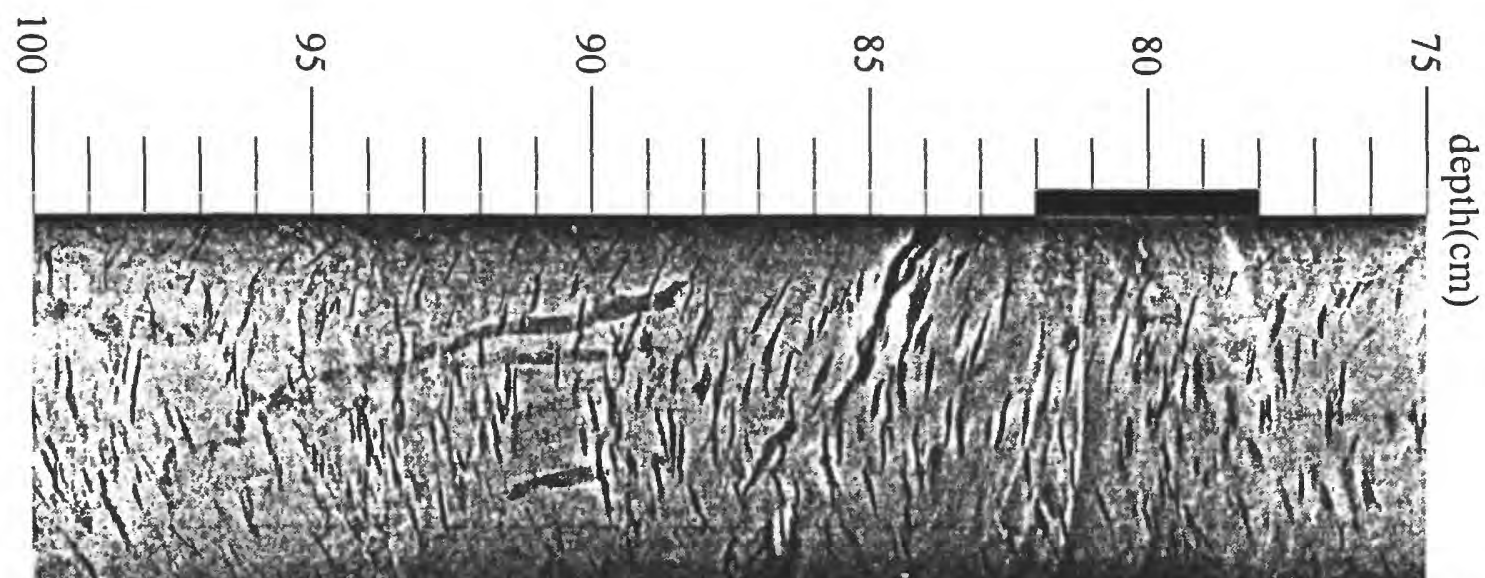

$a_{0}$

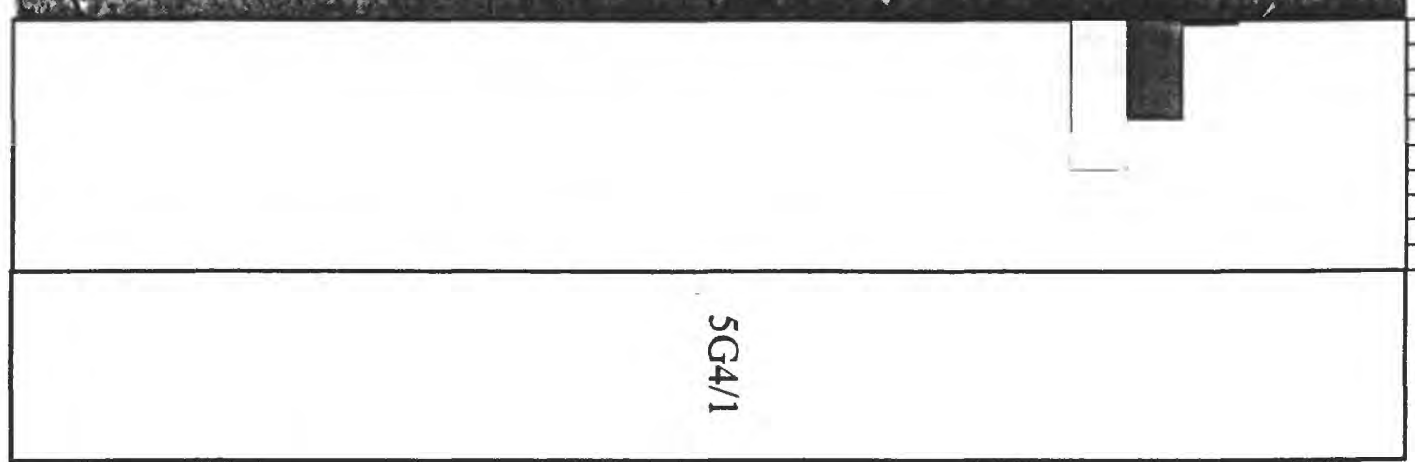

$=0$

夏

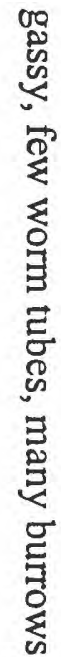

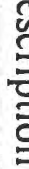



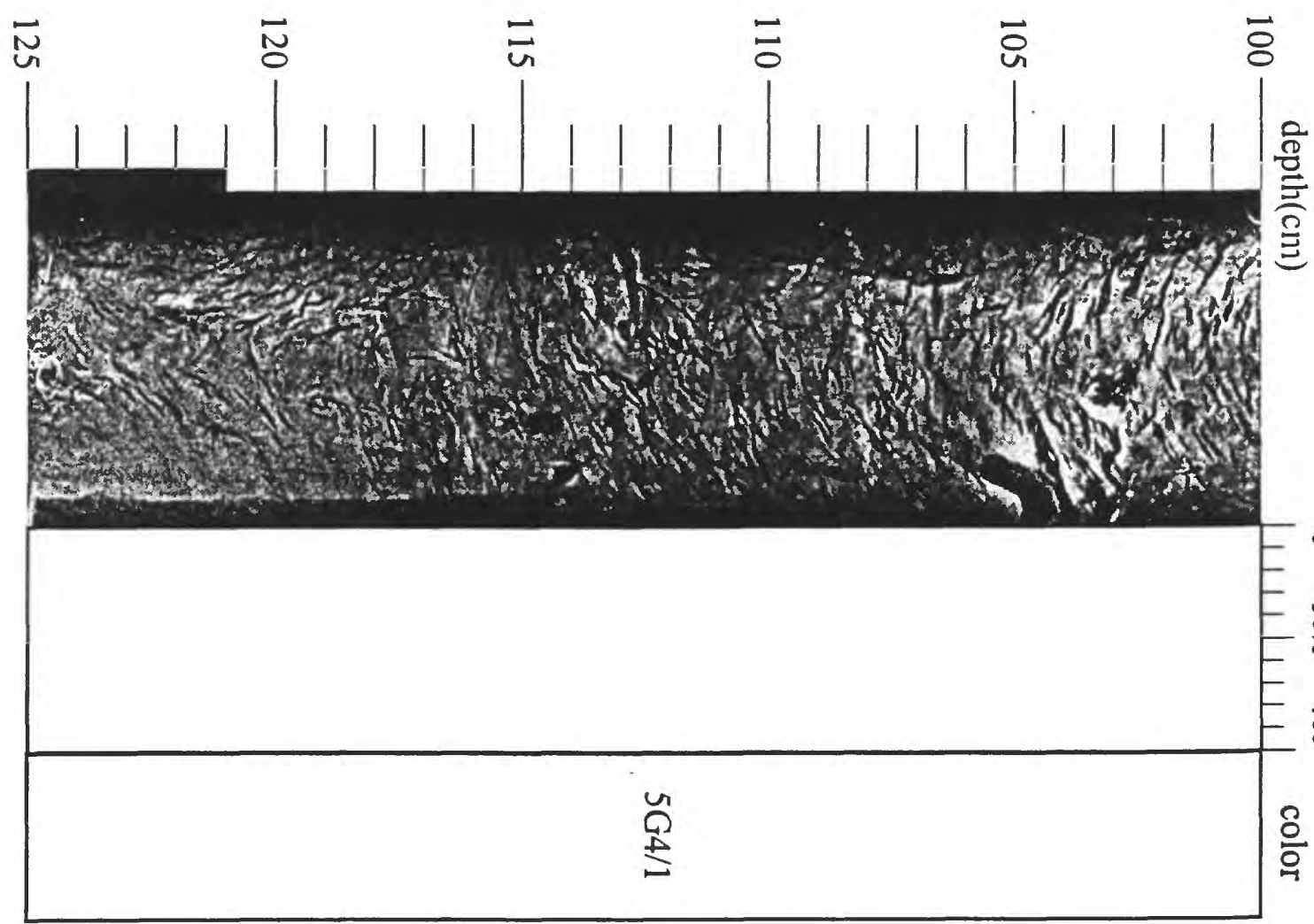

$=0$ - $=0$

壱

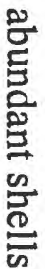

总家

กํํㅇ

응.

总营

的完

官寻

일

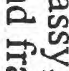

骂 을

总

芑

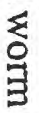

四 


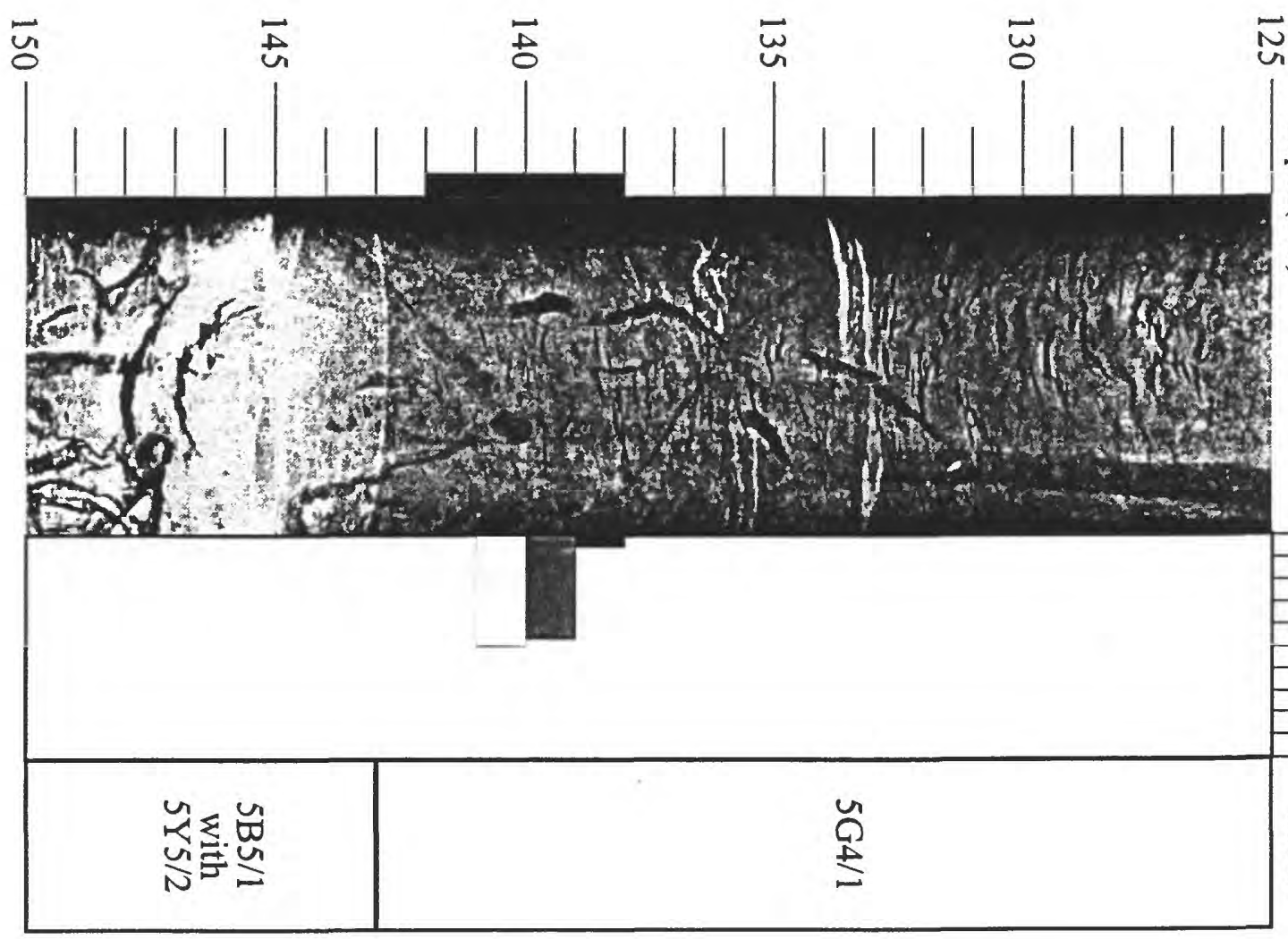

高

$x$

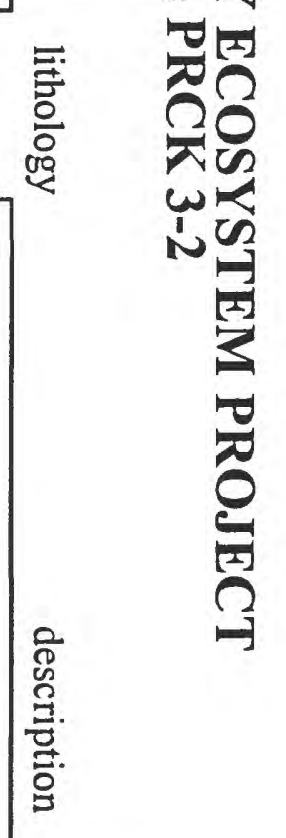




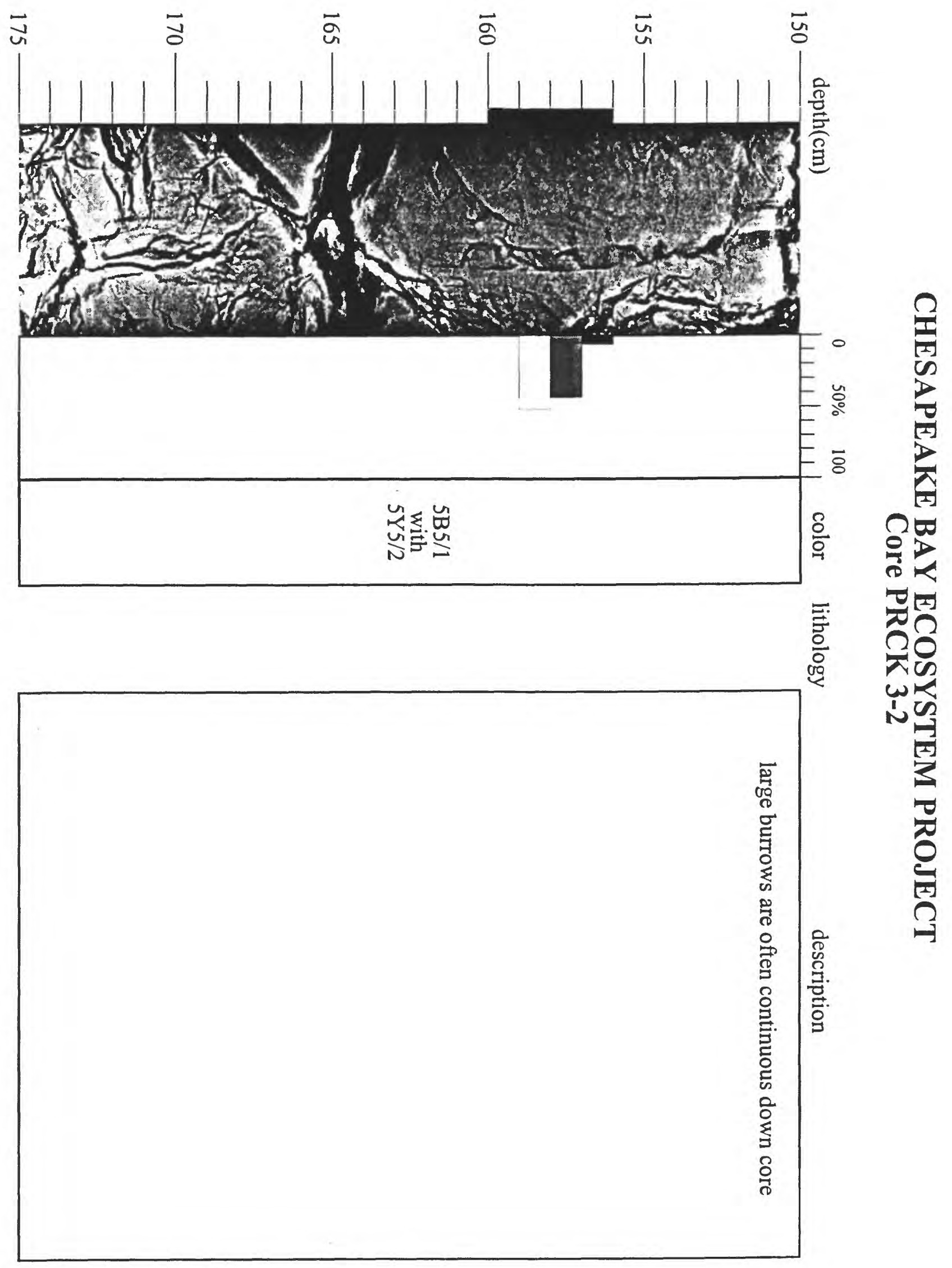



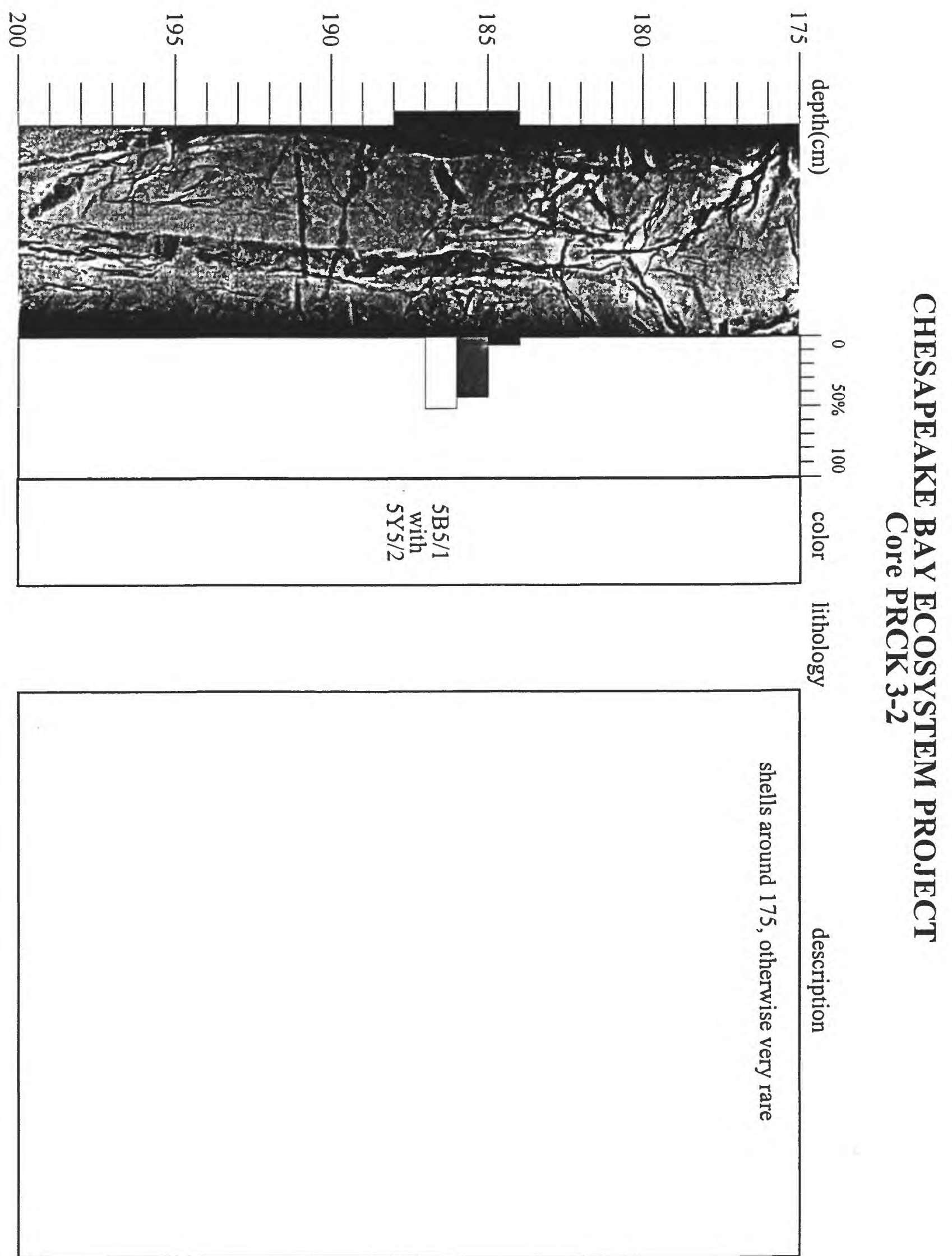

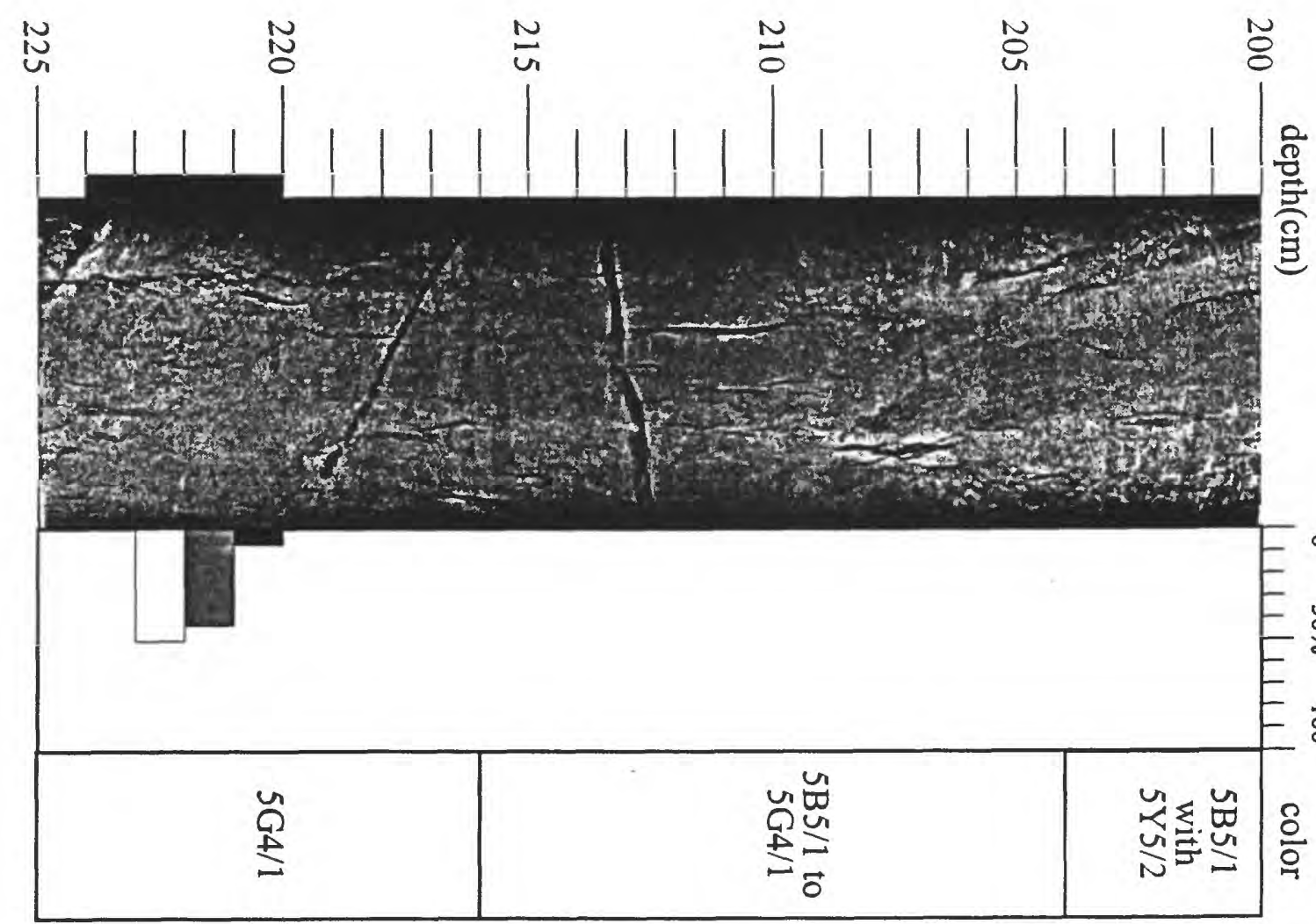

$\frac{1}{w}$

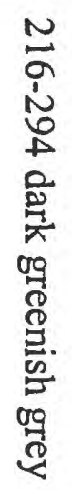

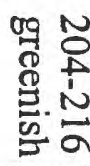

骂 㤐

焉

음

붕

范

号

它

品

$\overrightarrow{0}$

$=0$

흥

응

Rेo

ws

[a 


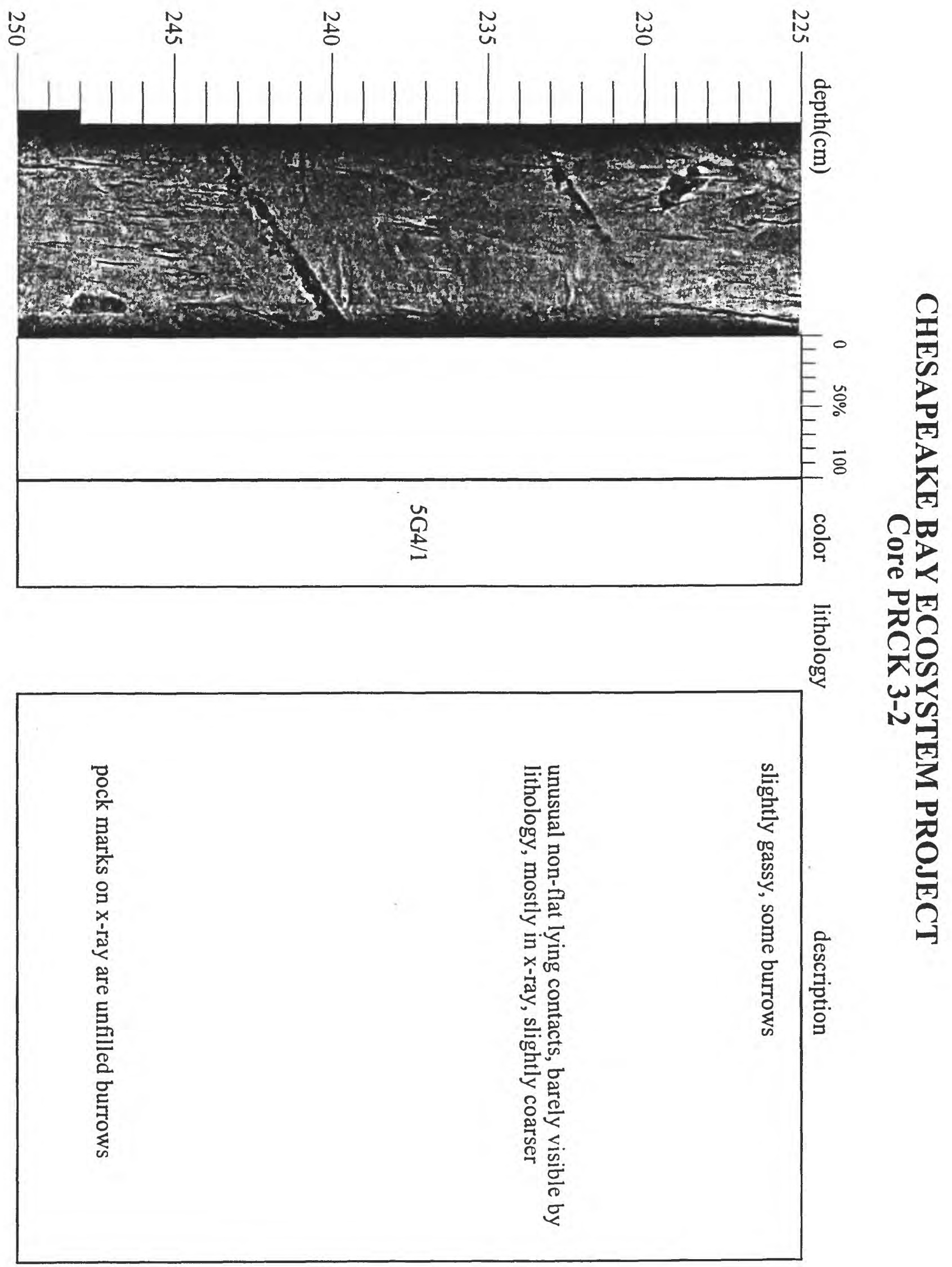




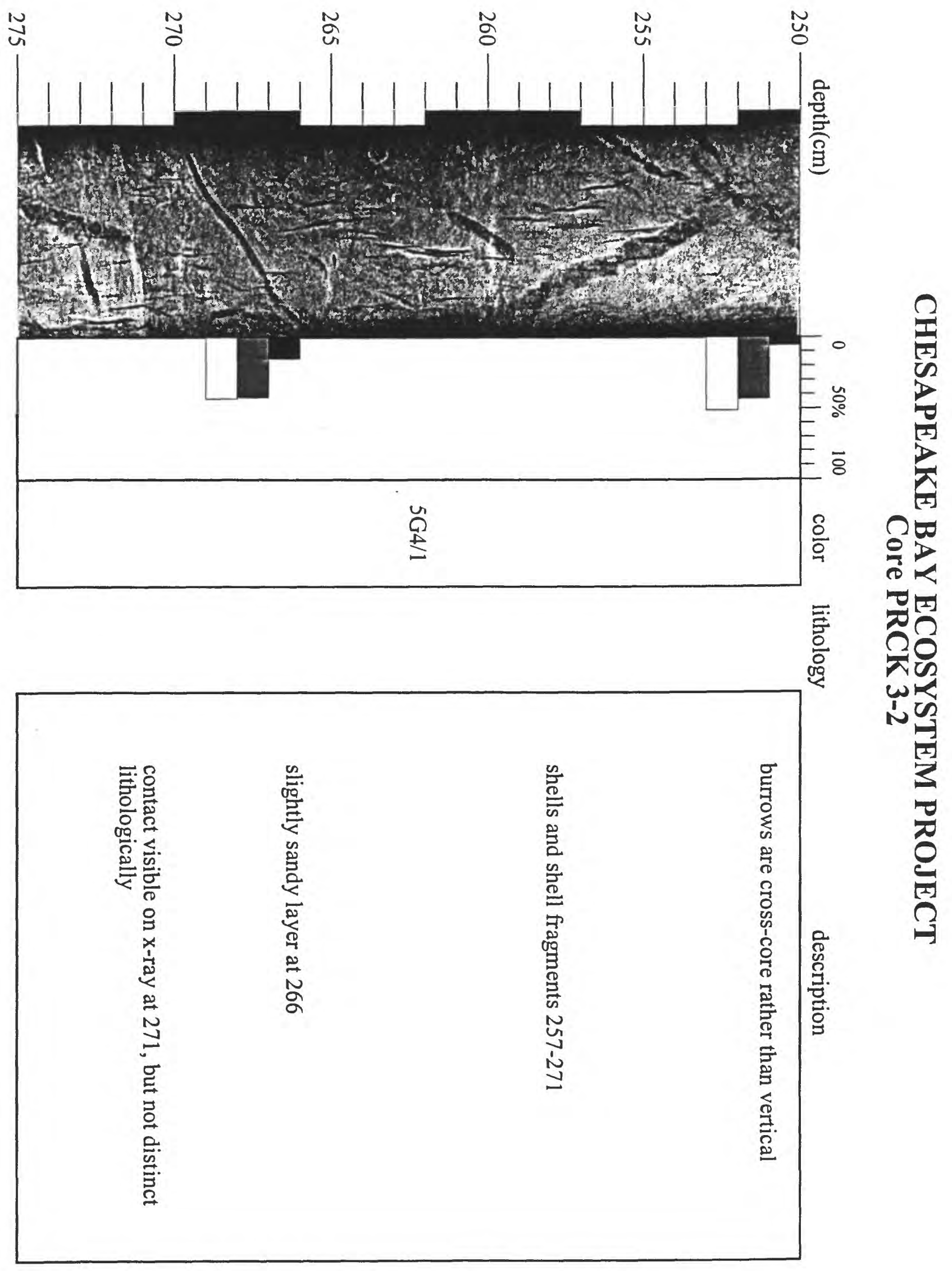



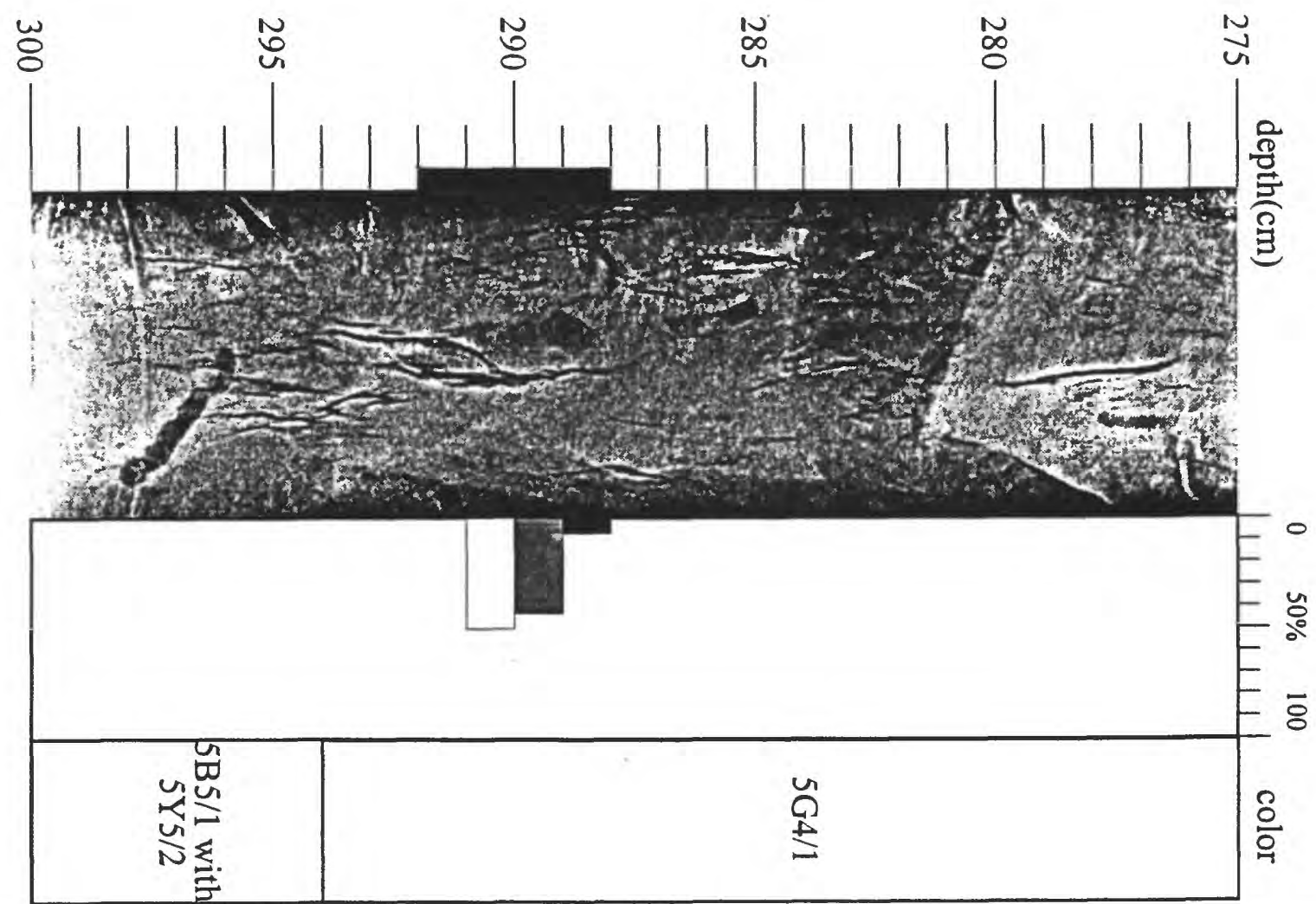

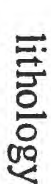

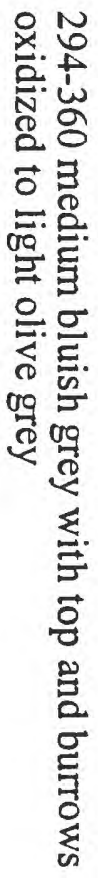

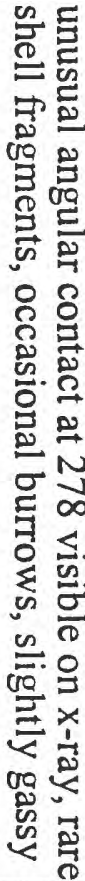

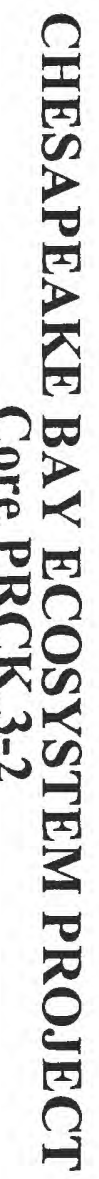




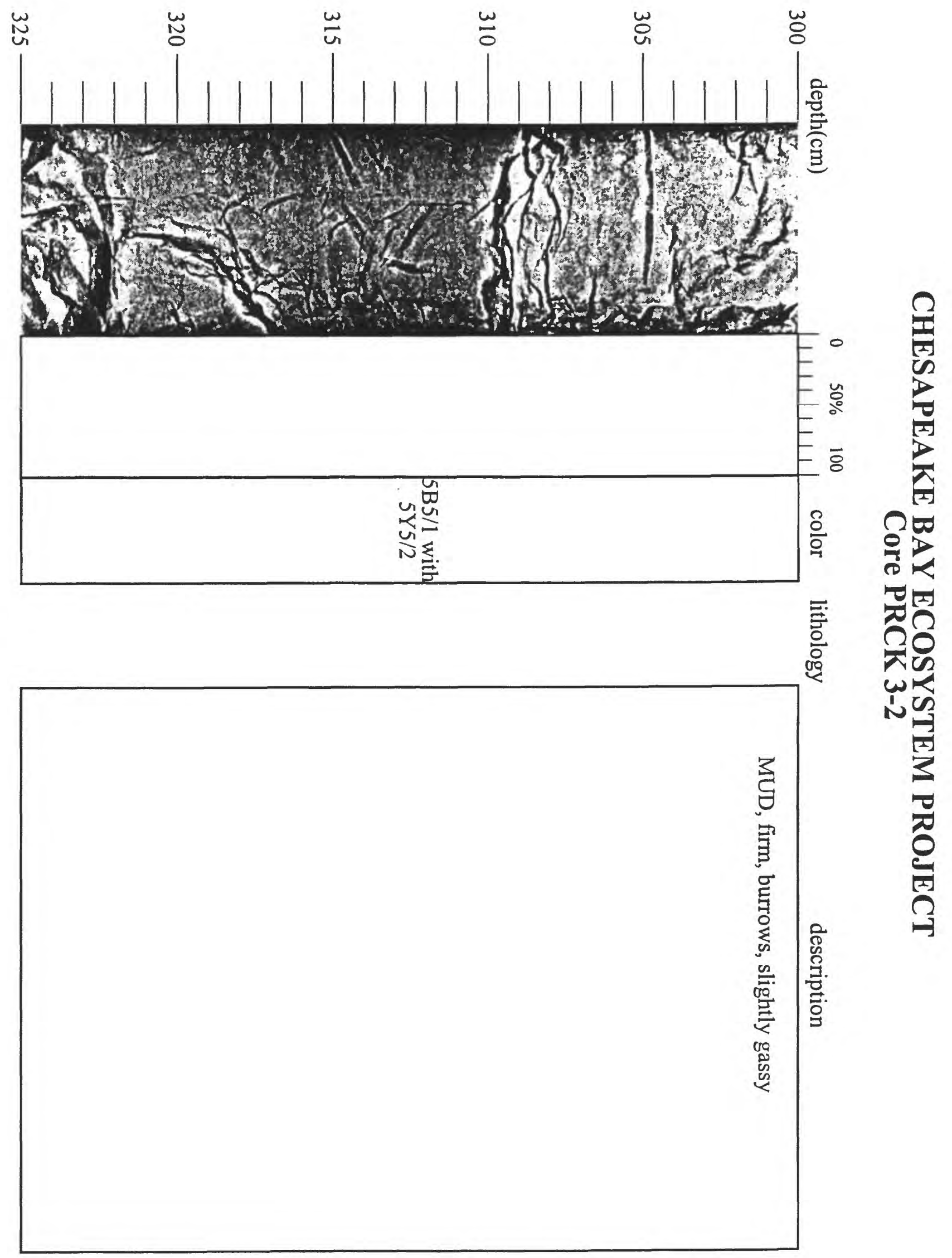




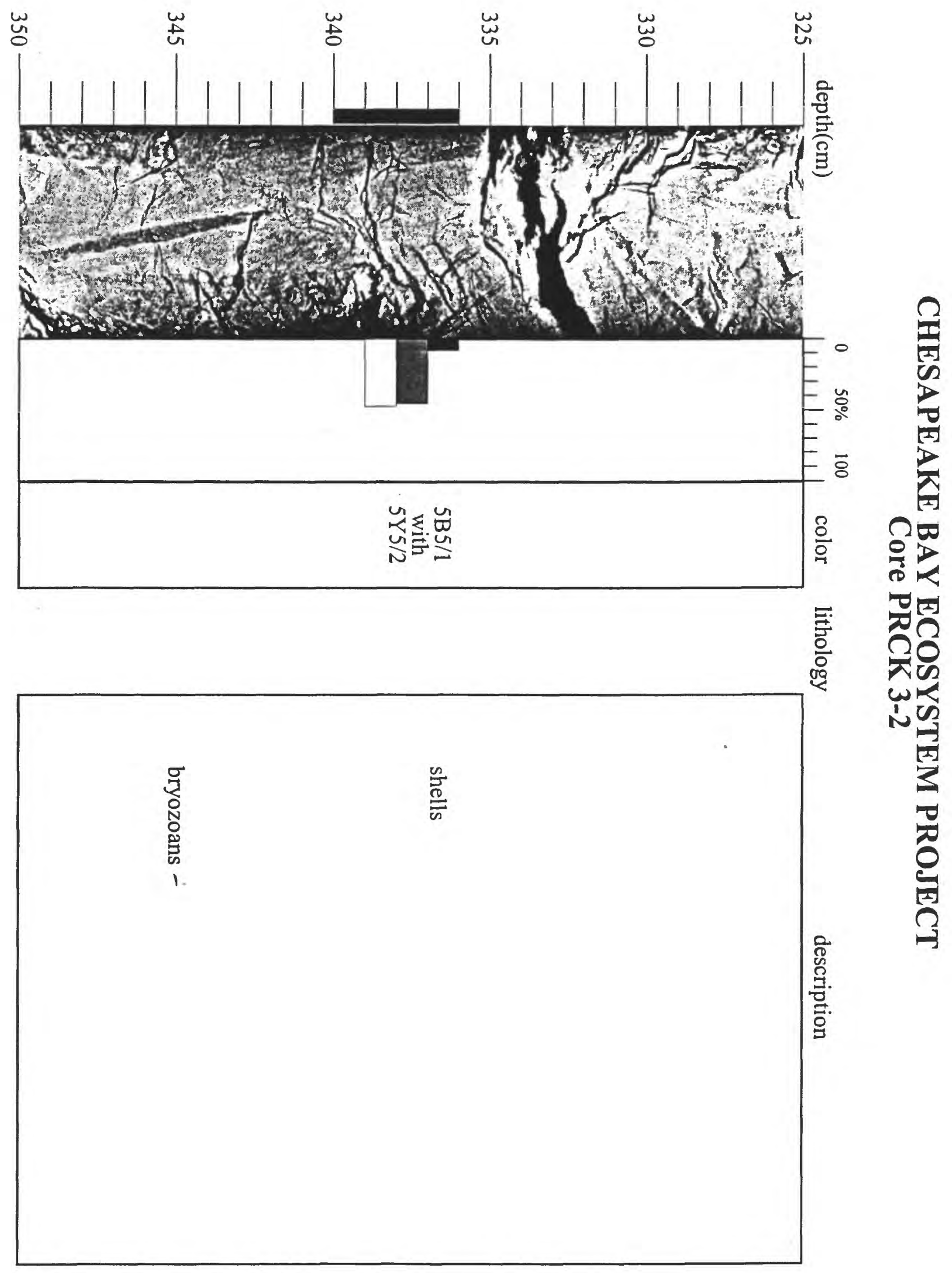




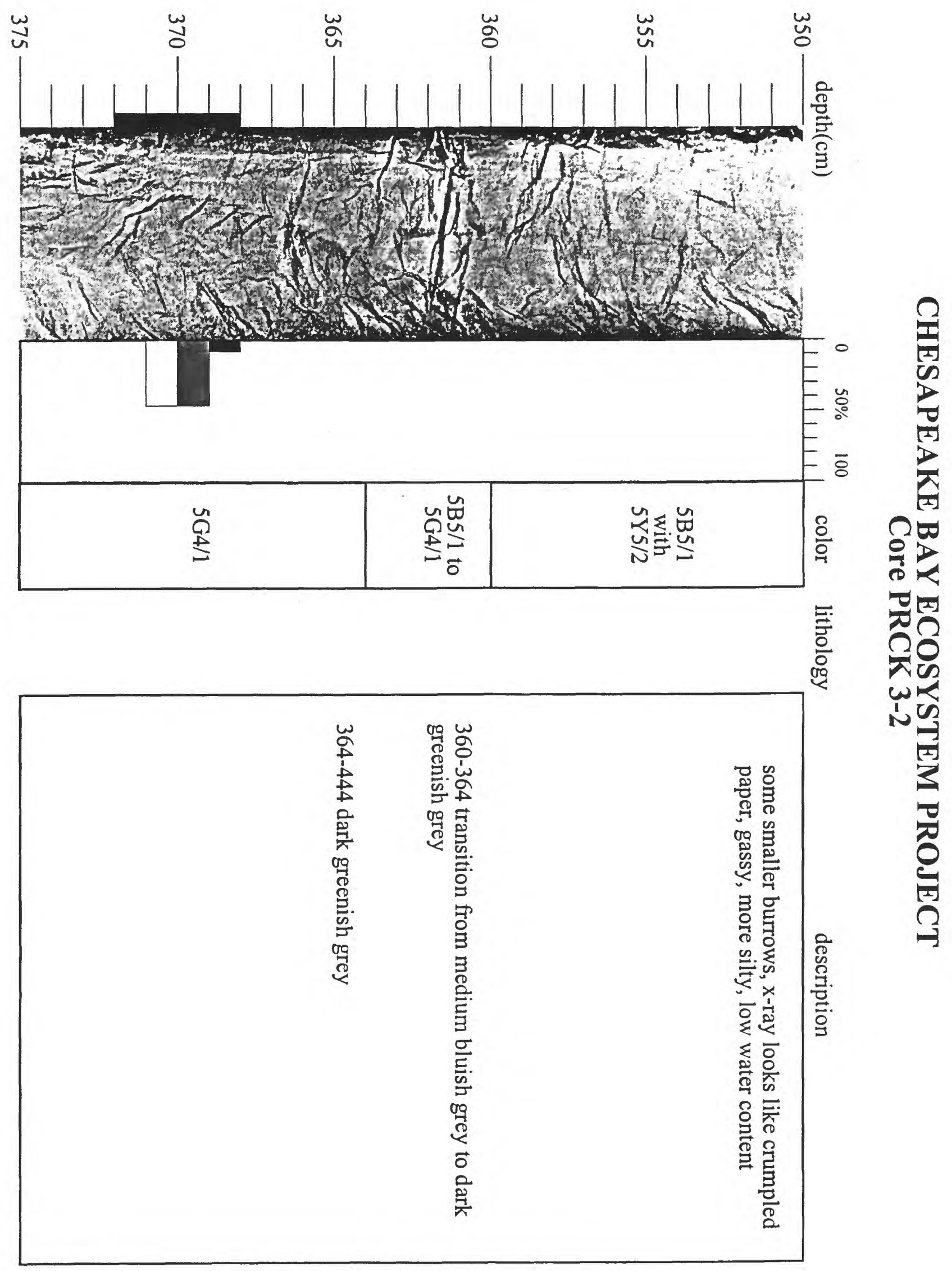



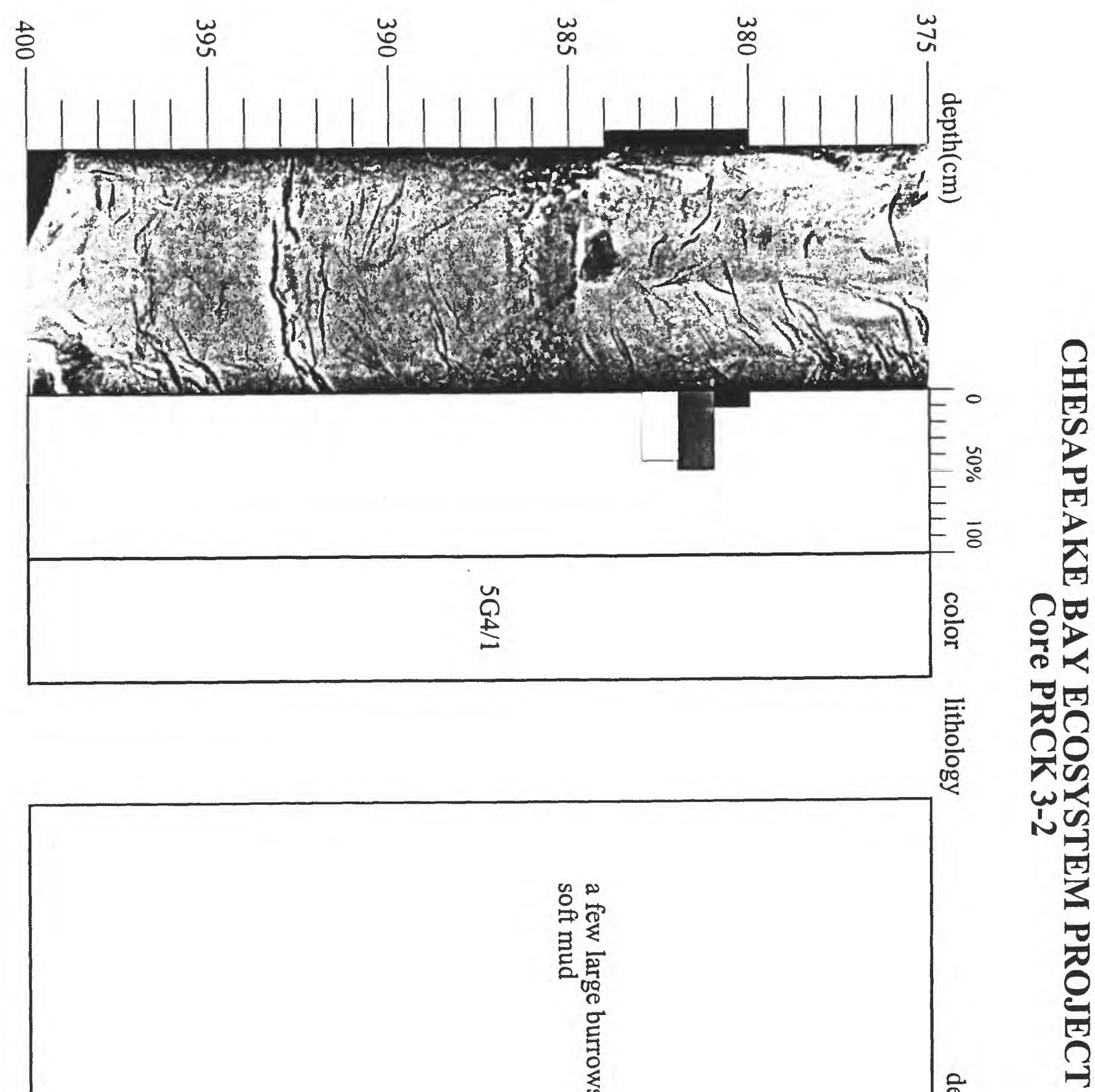


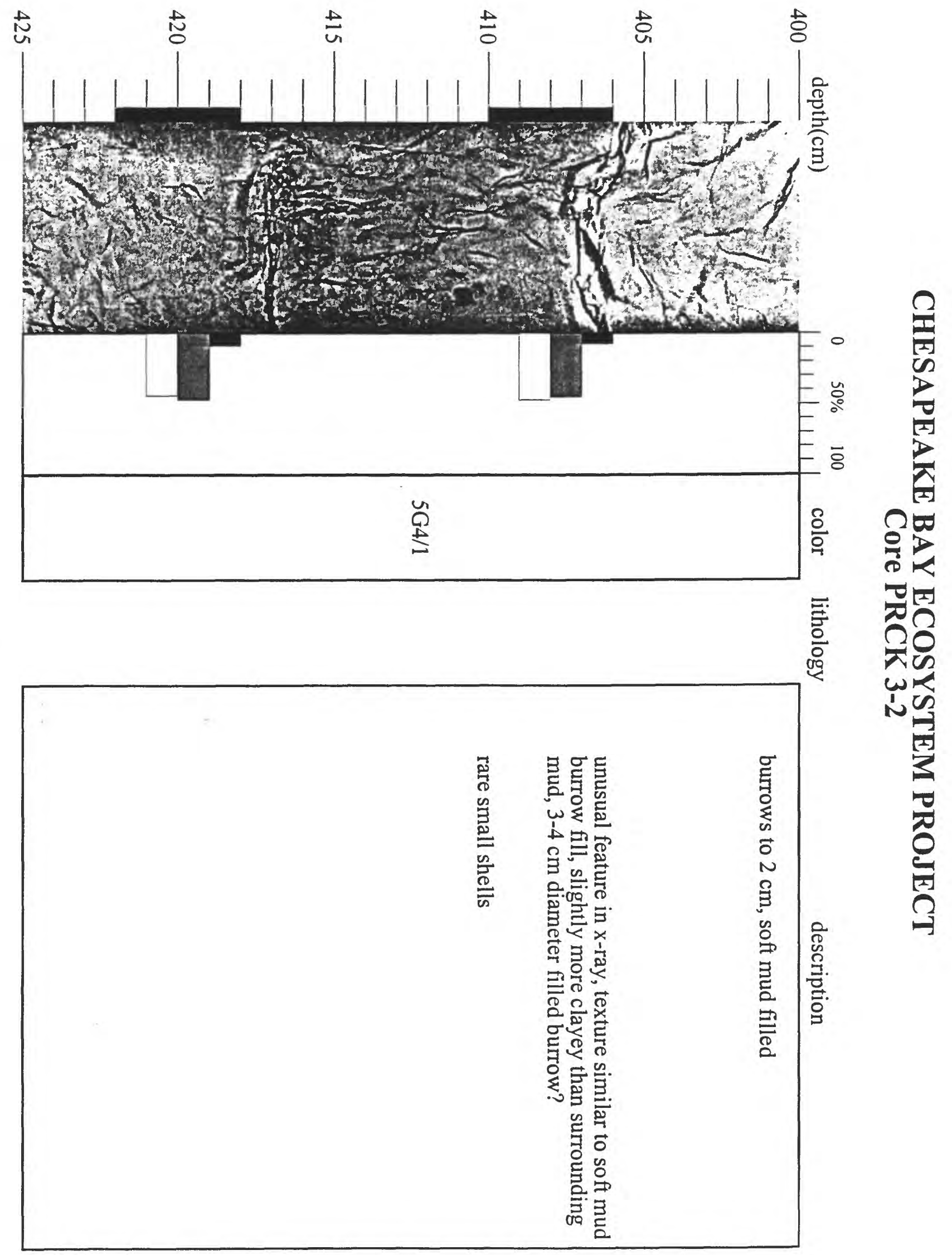




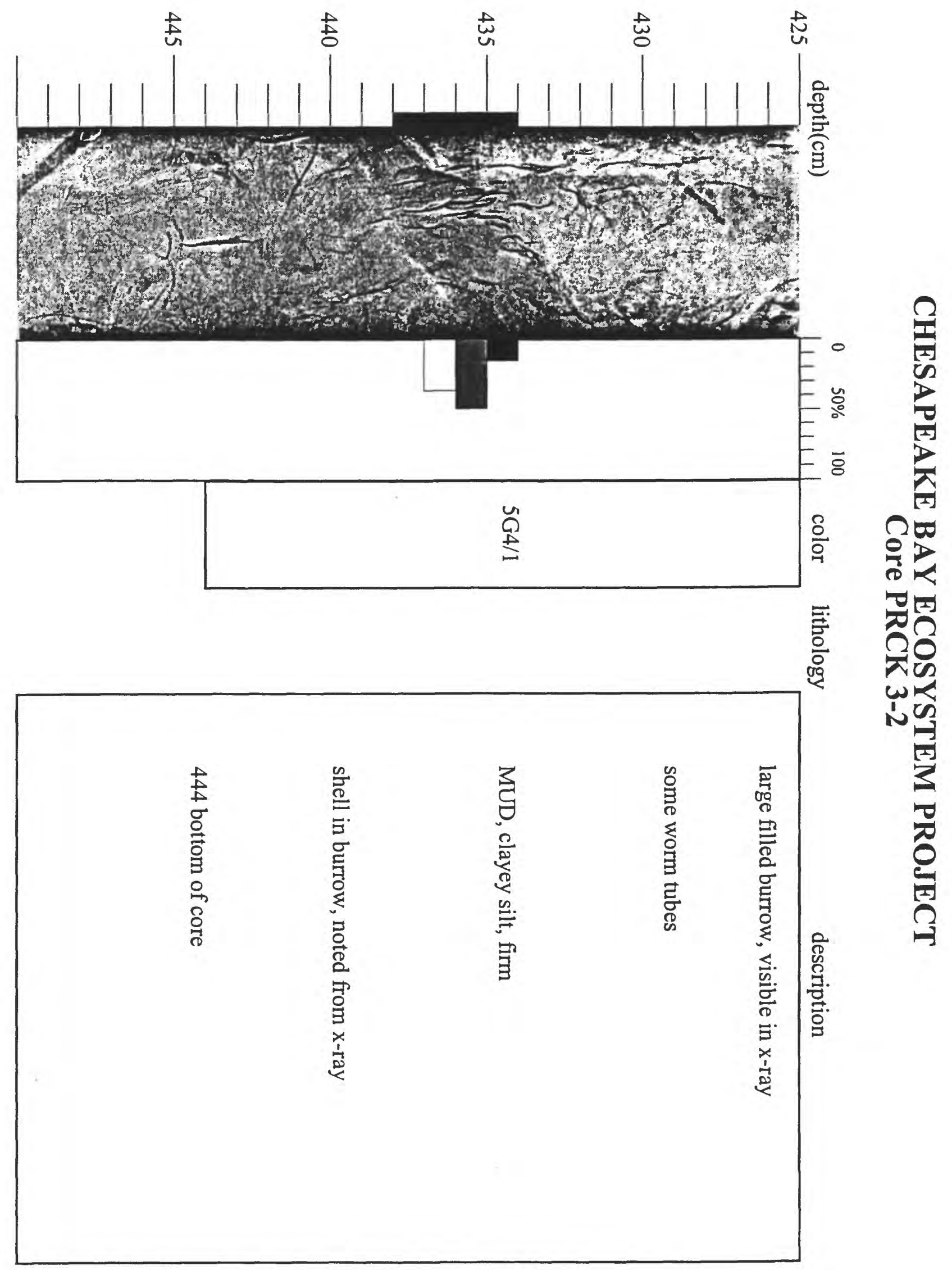




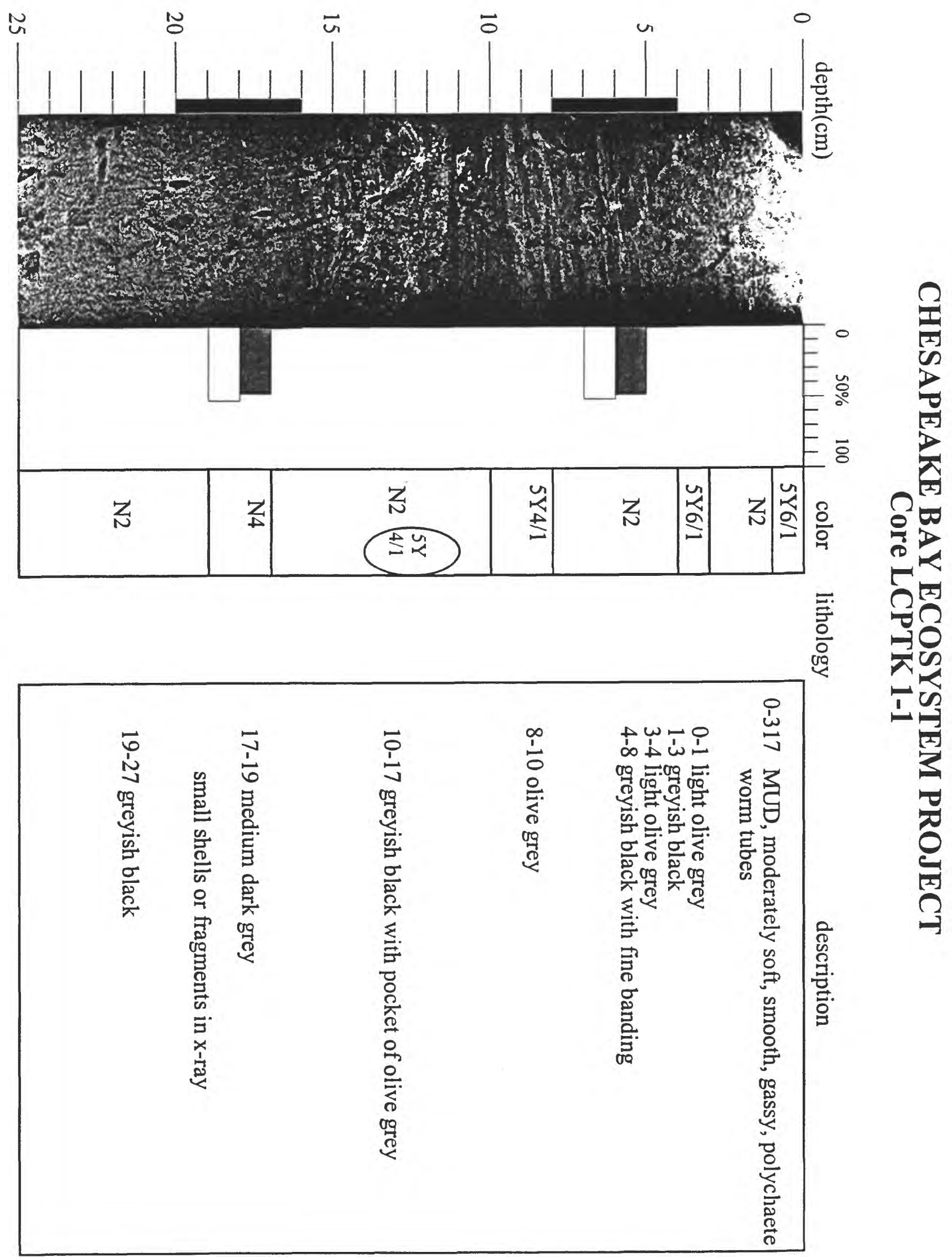




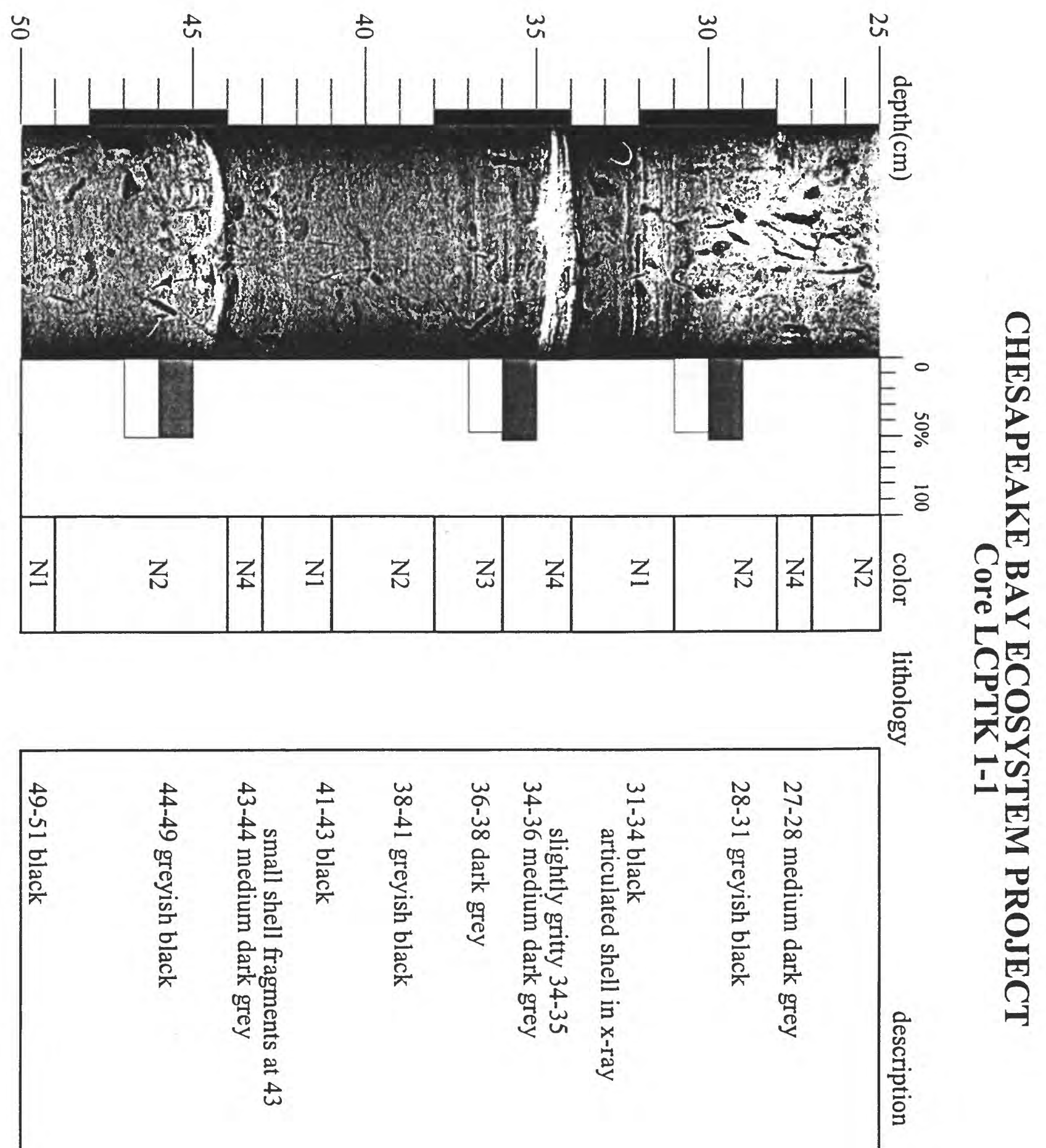




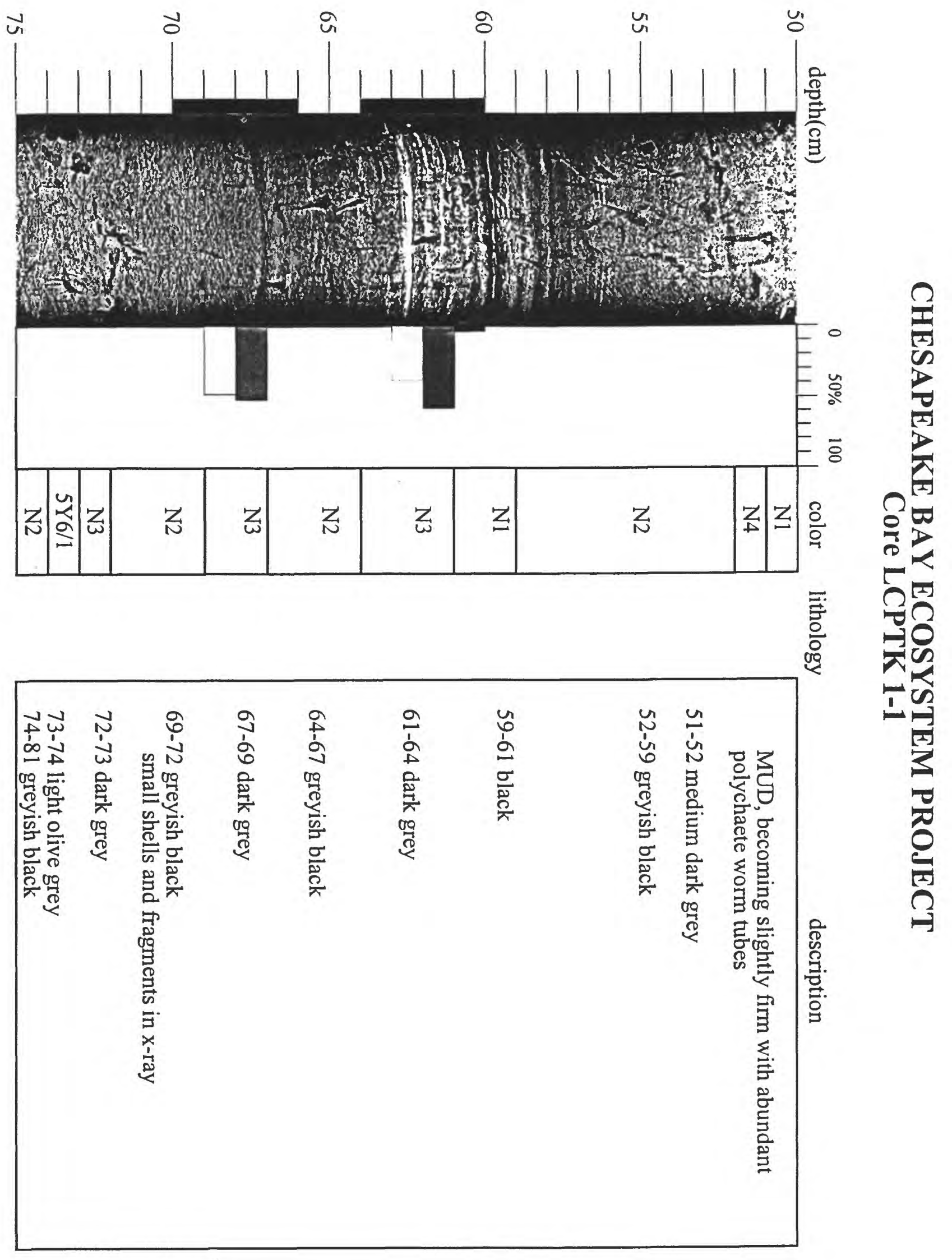



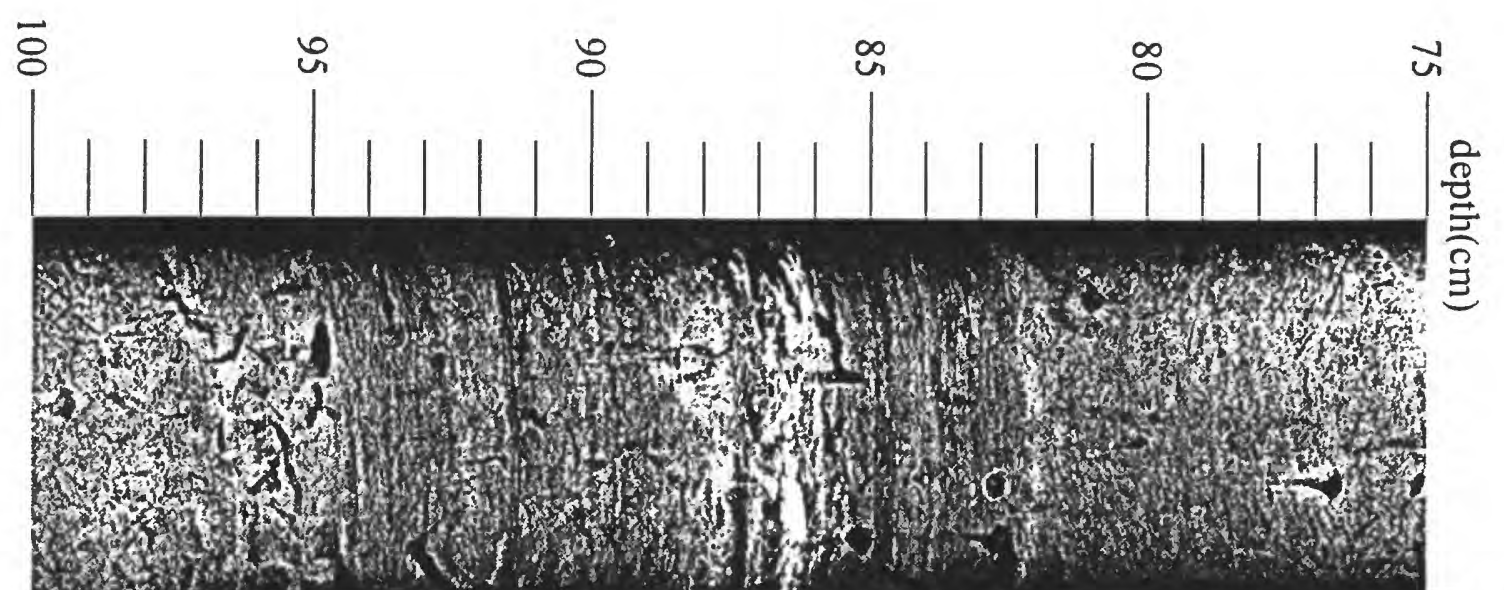

4

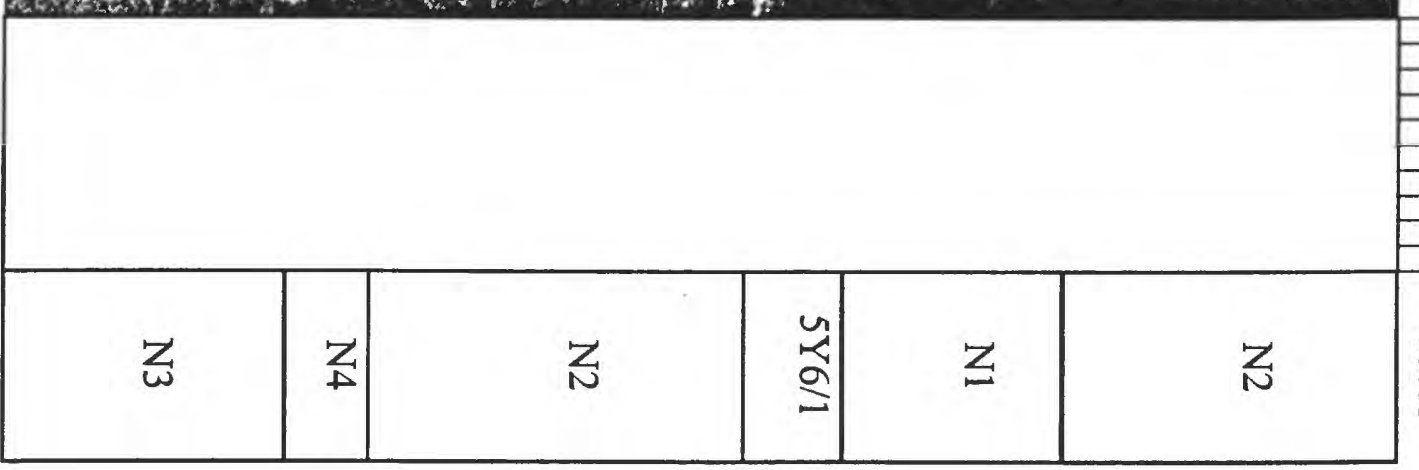

$=0$

=

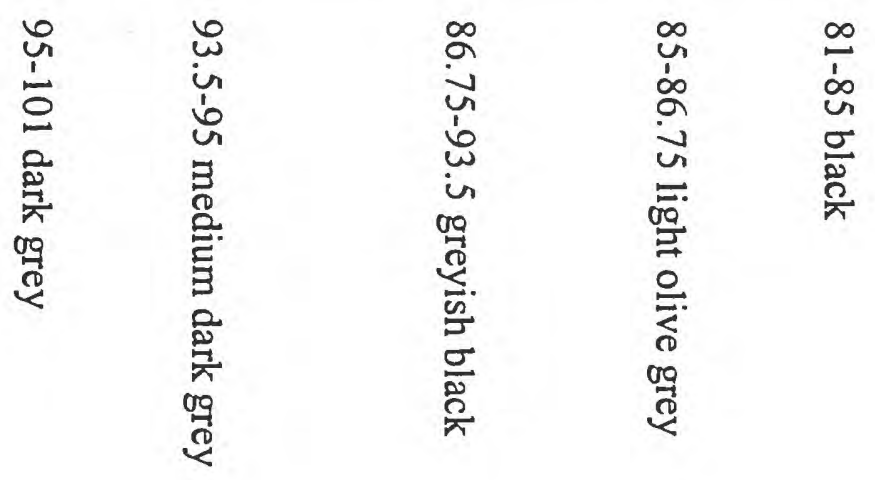

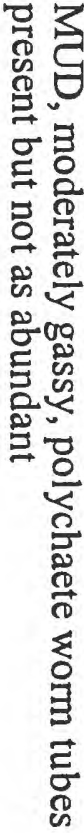




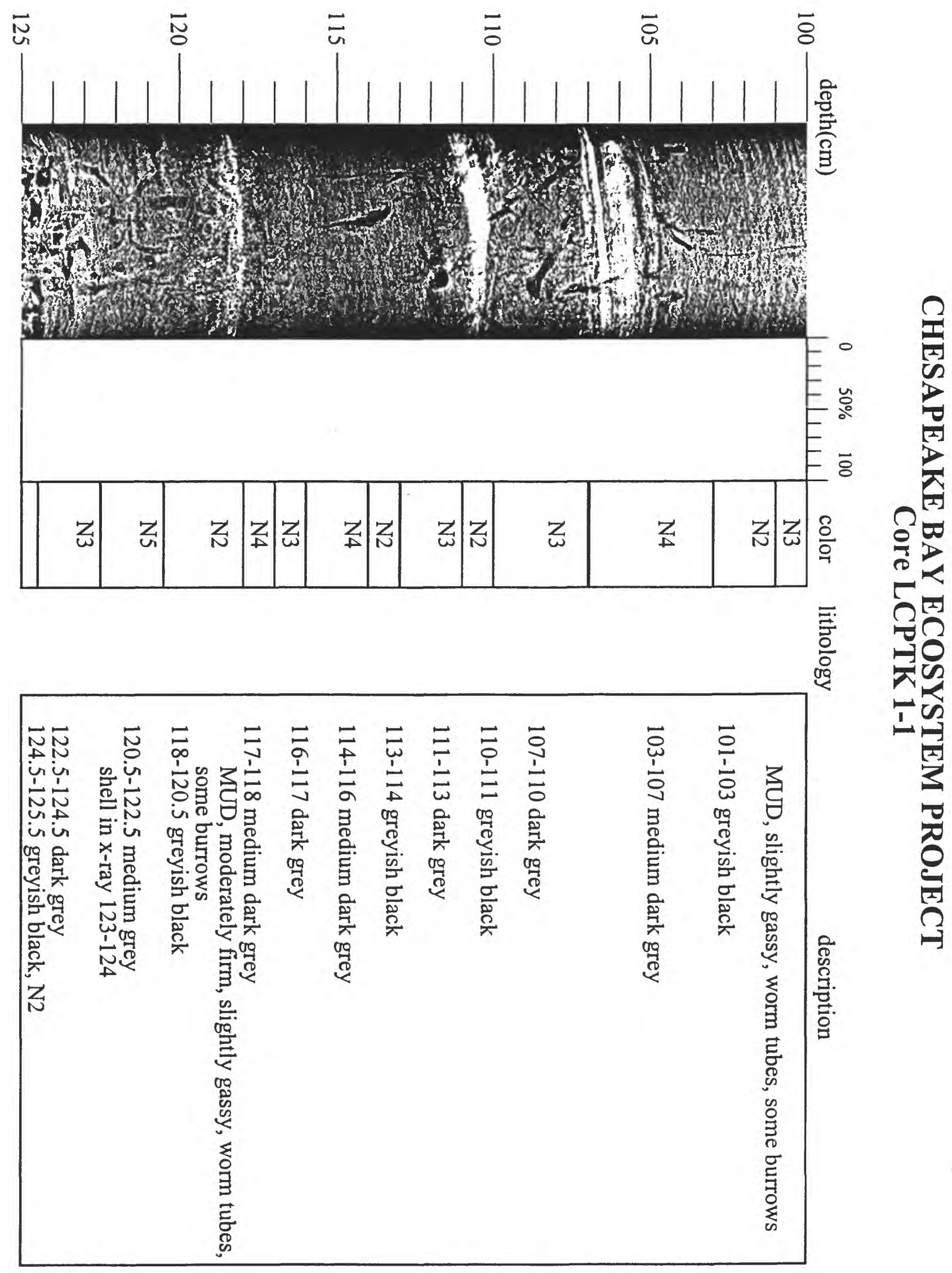




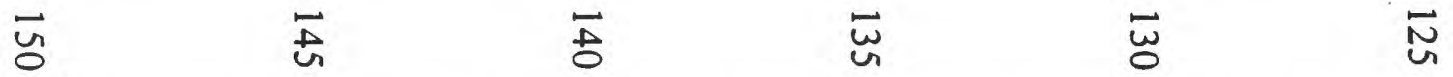
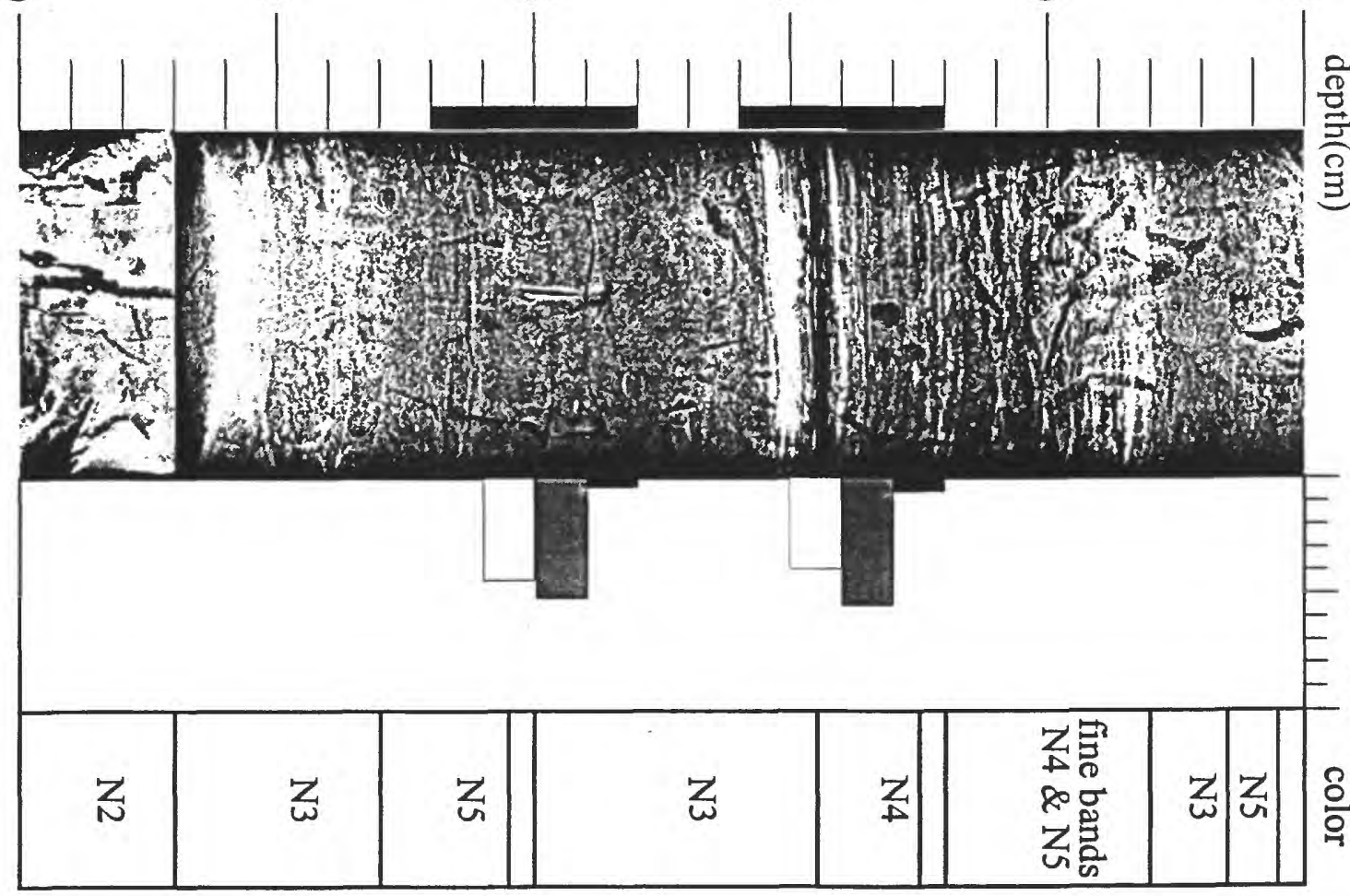

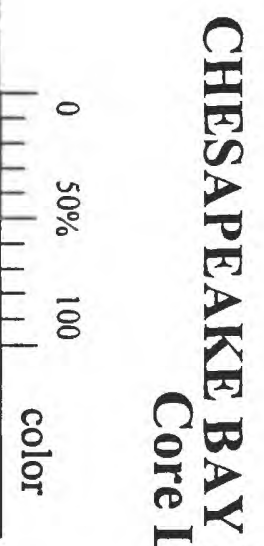




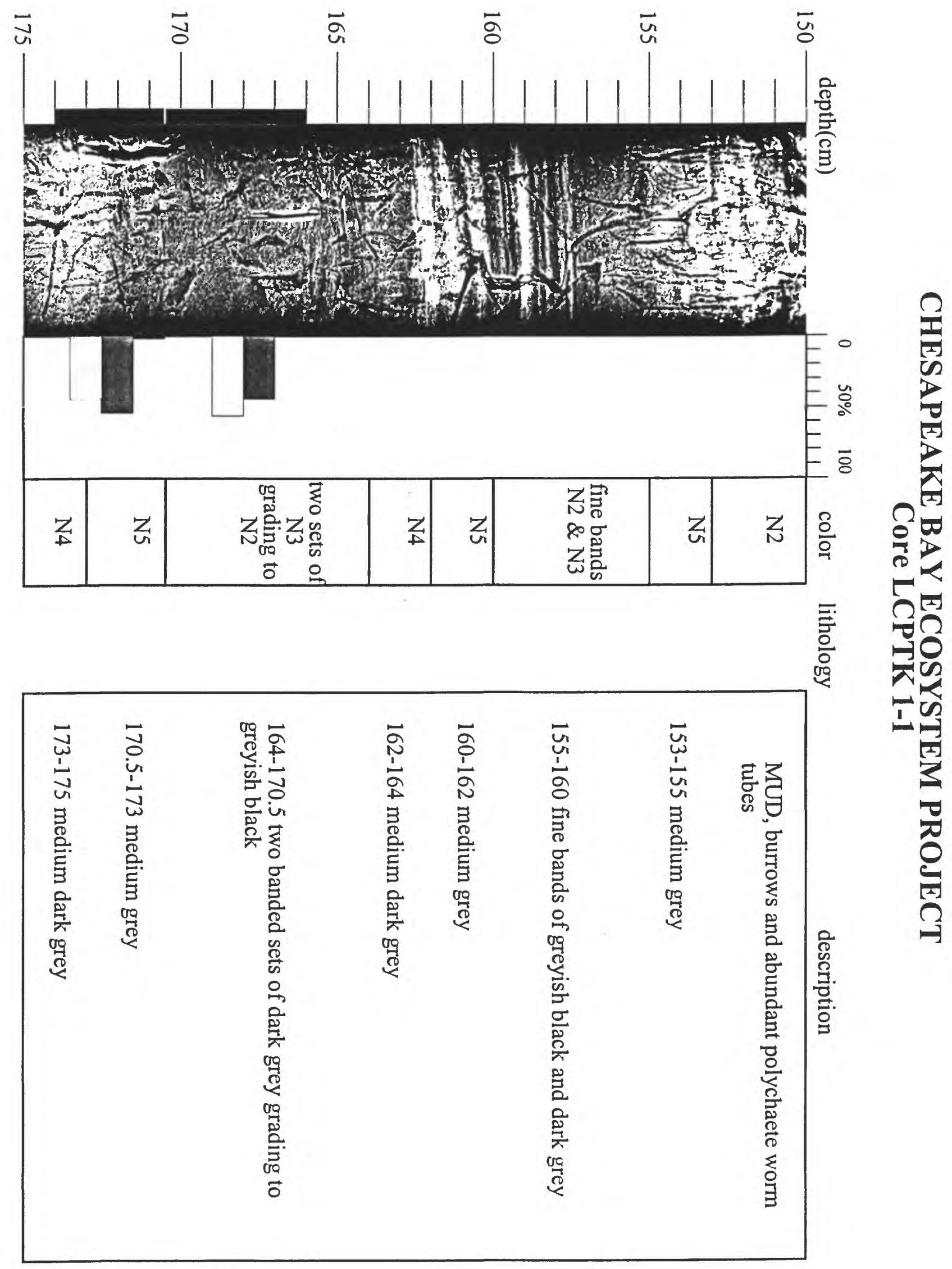



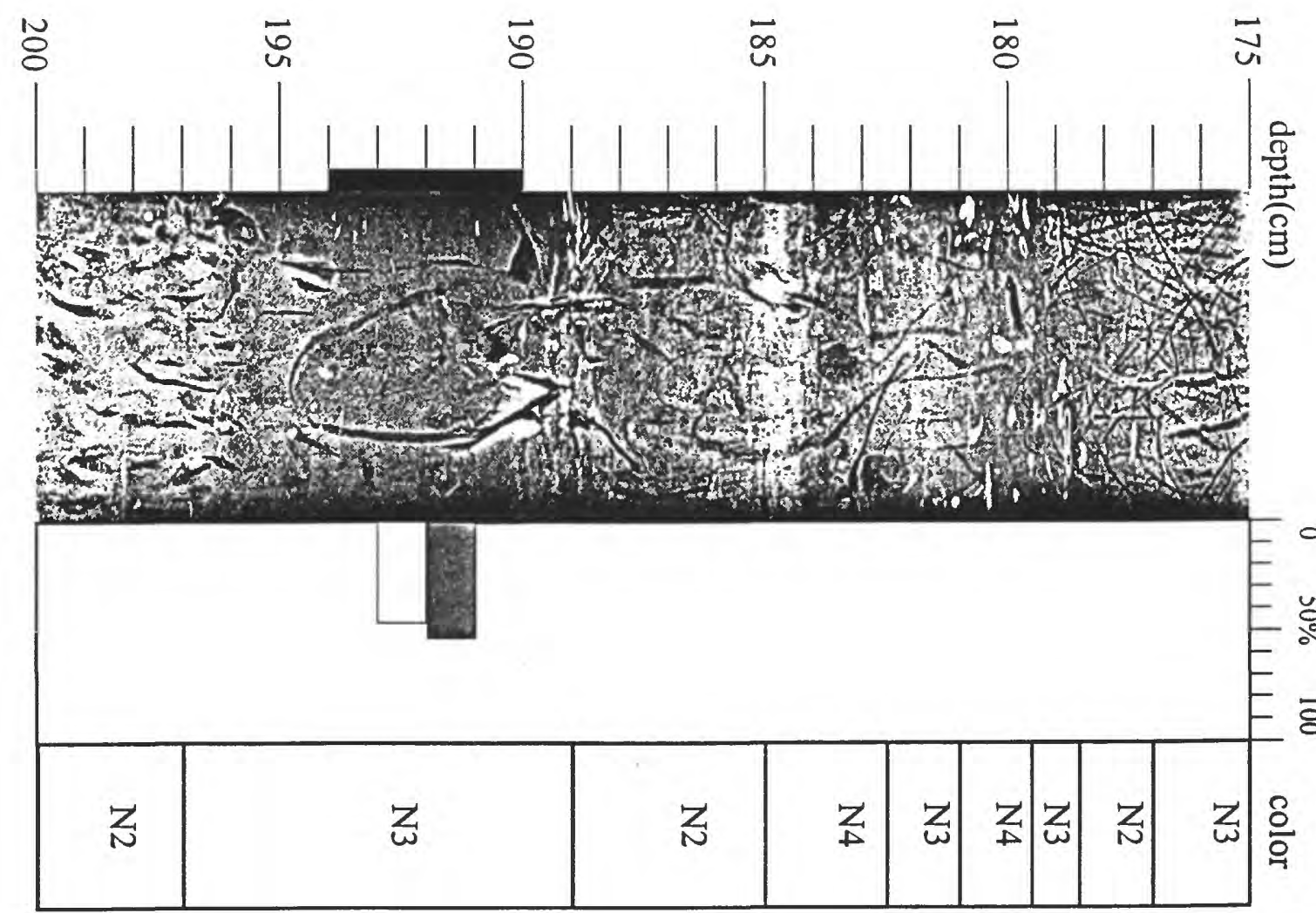

$=0$

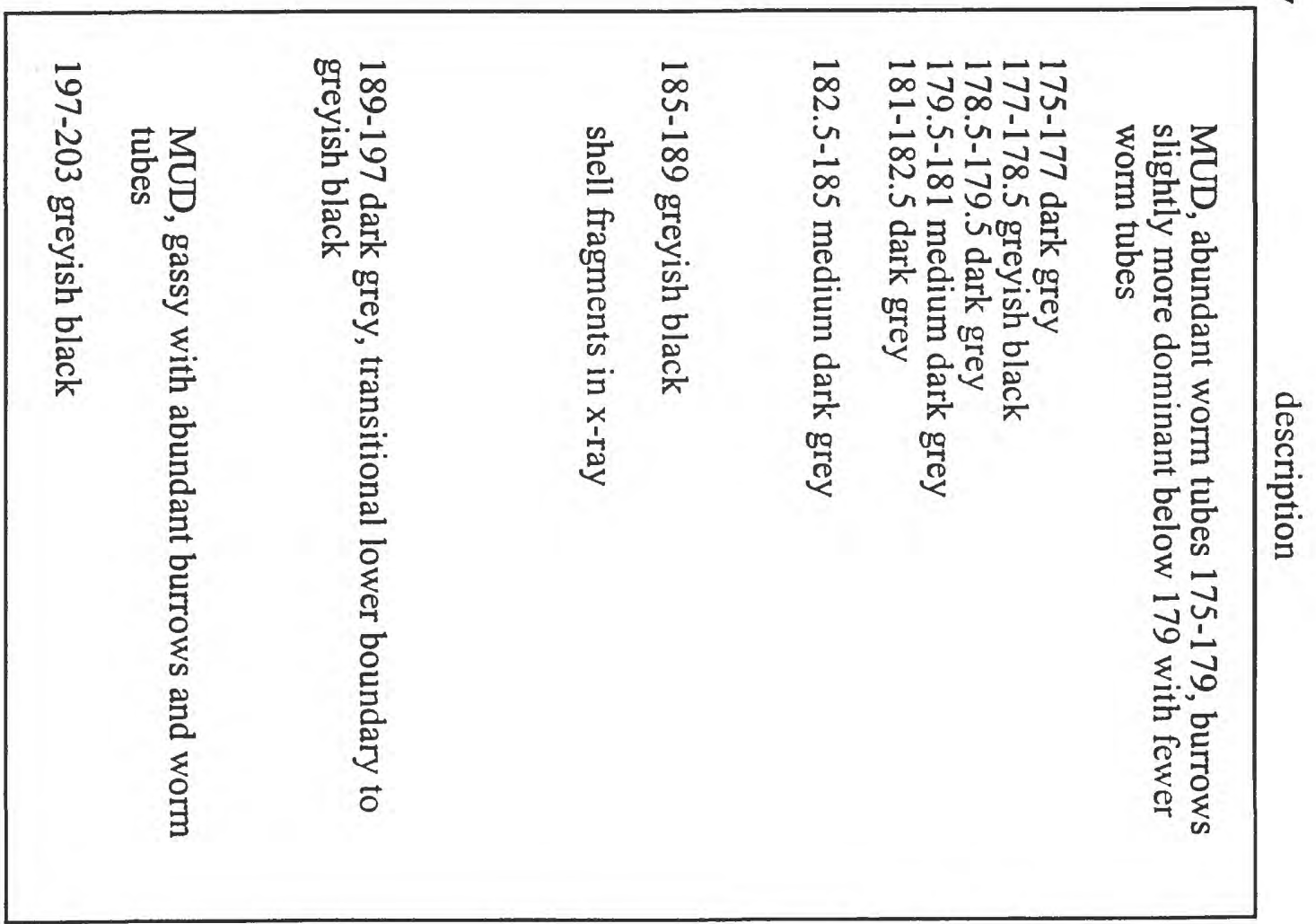




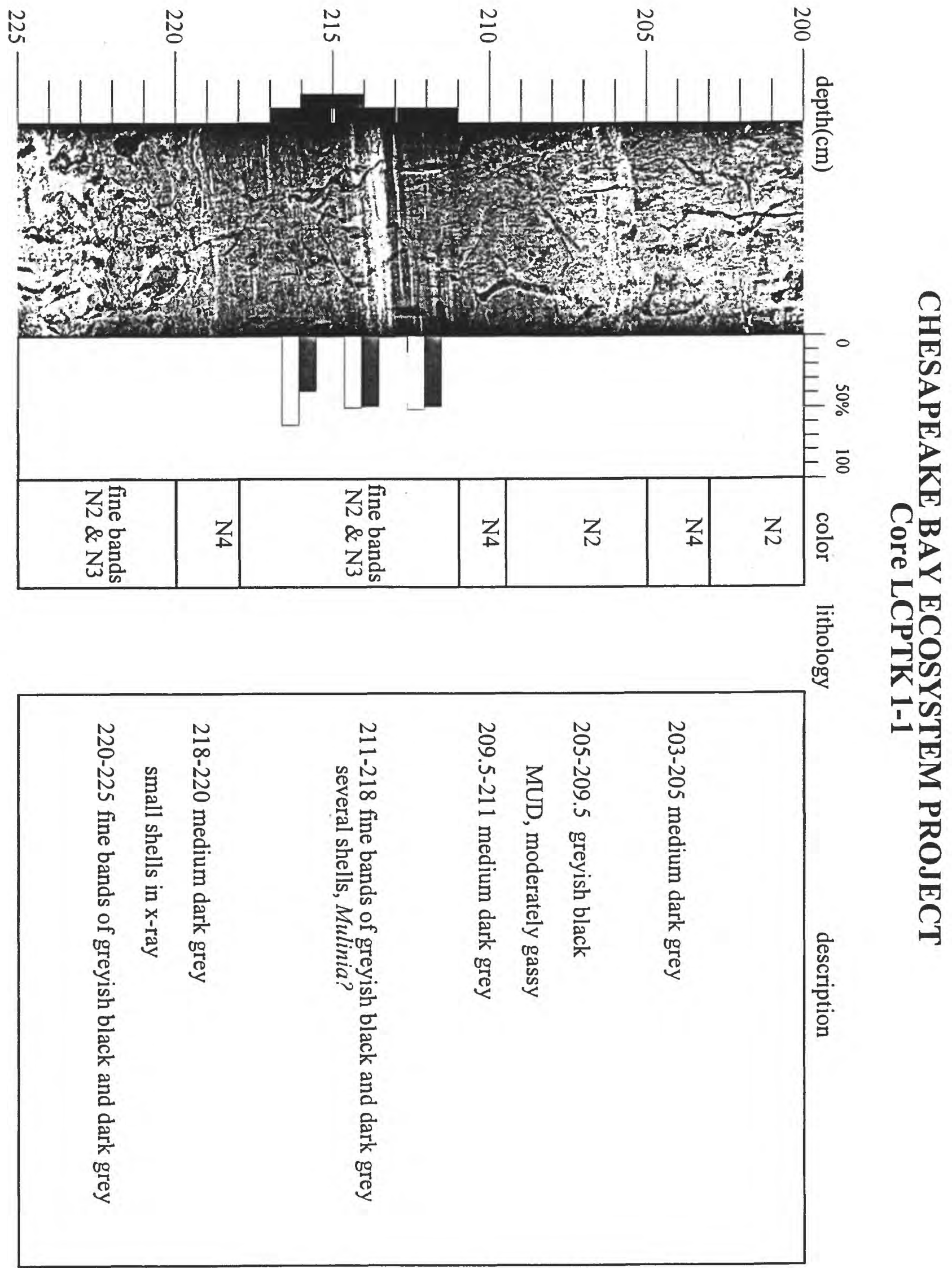




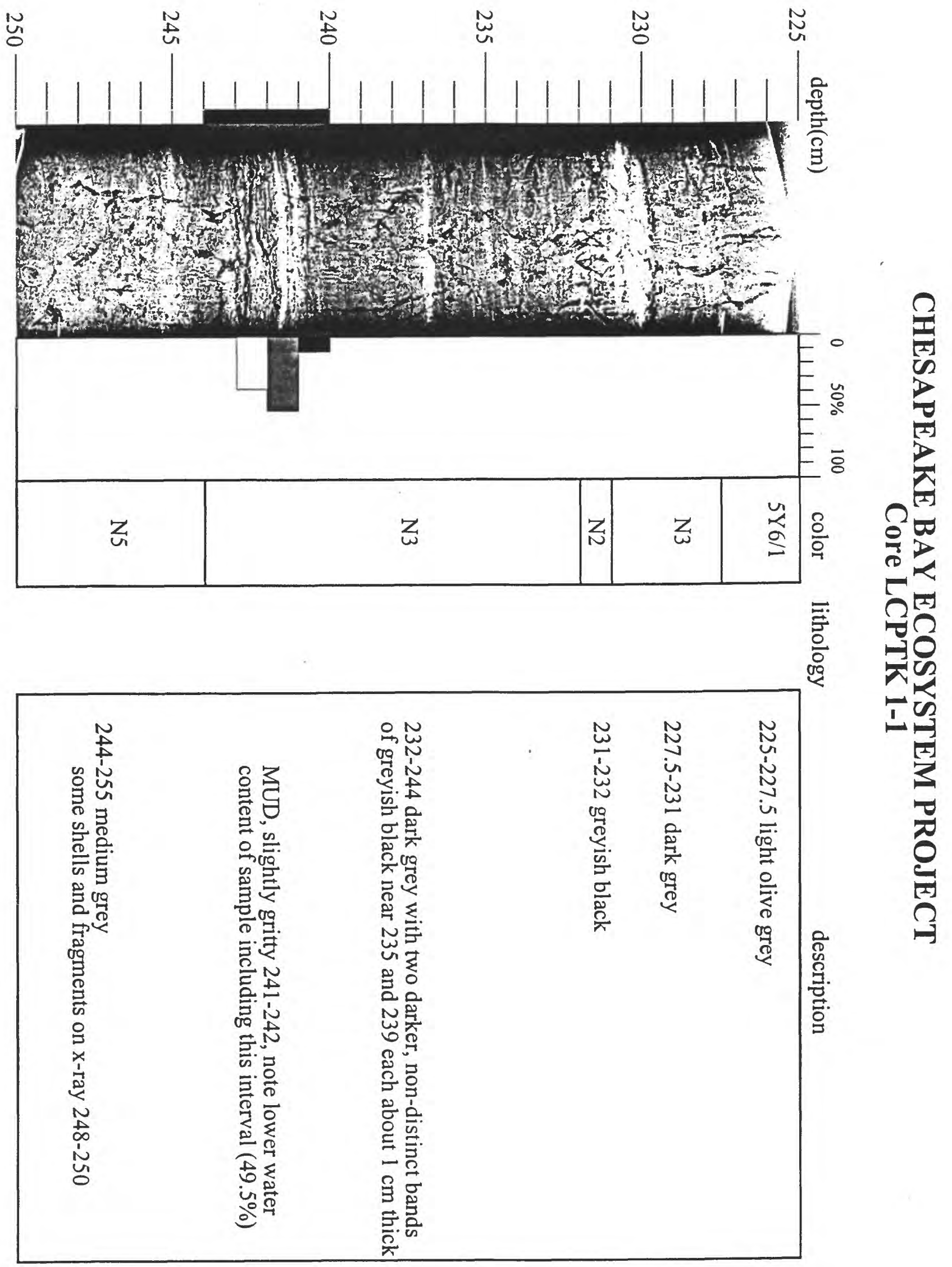




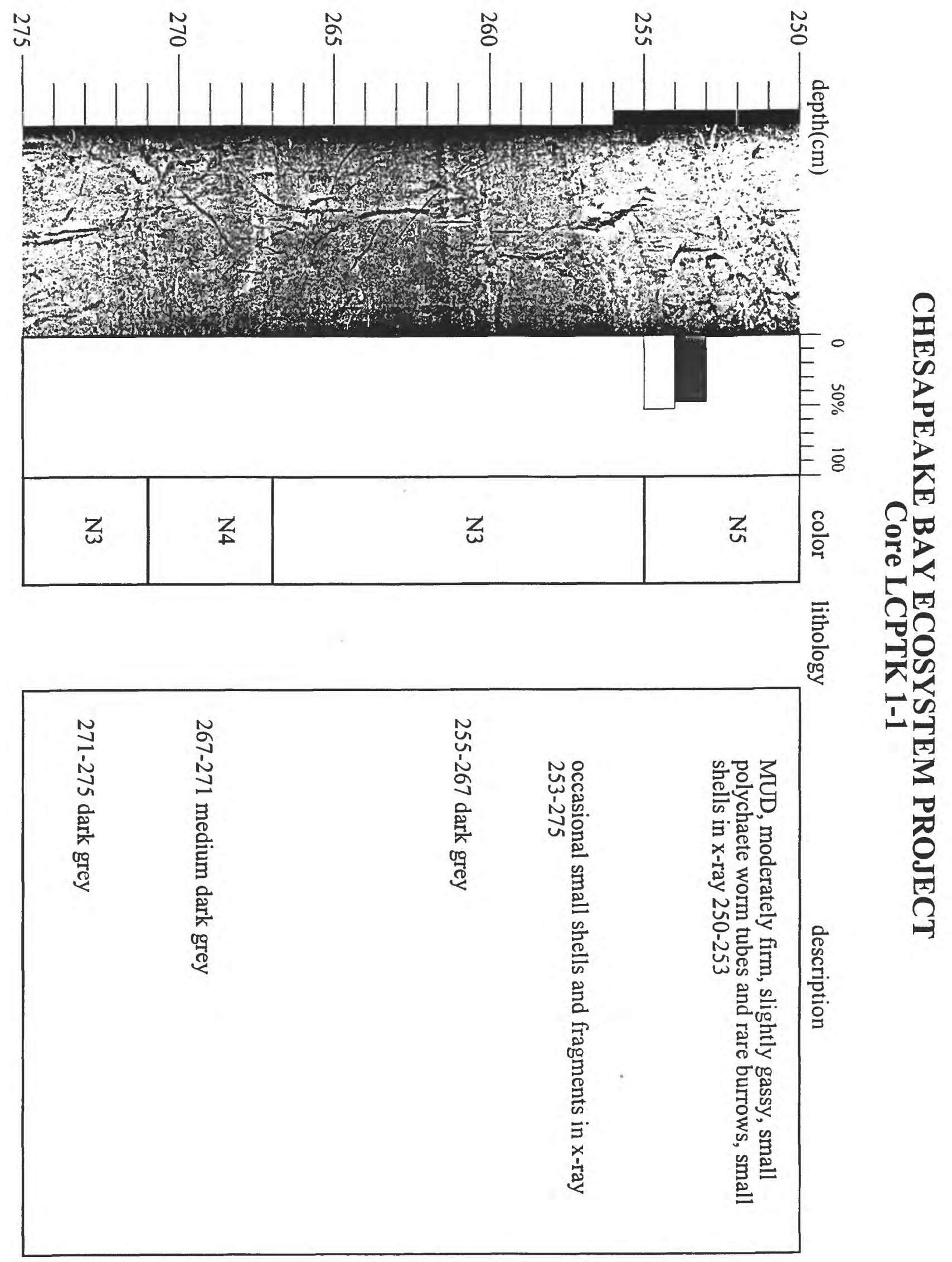




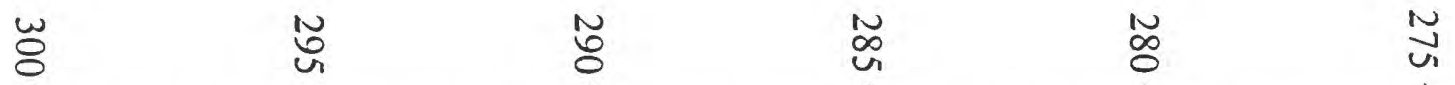

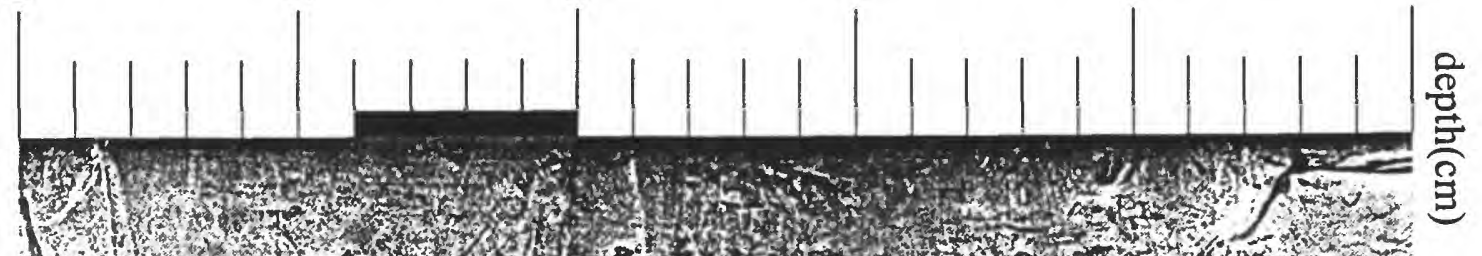

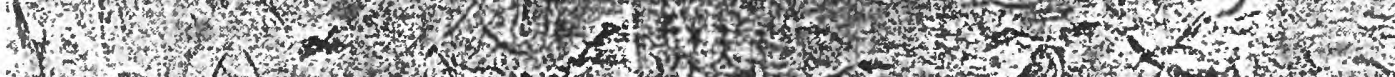
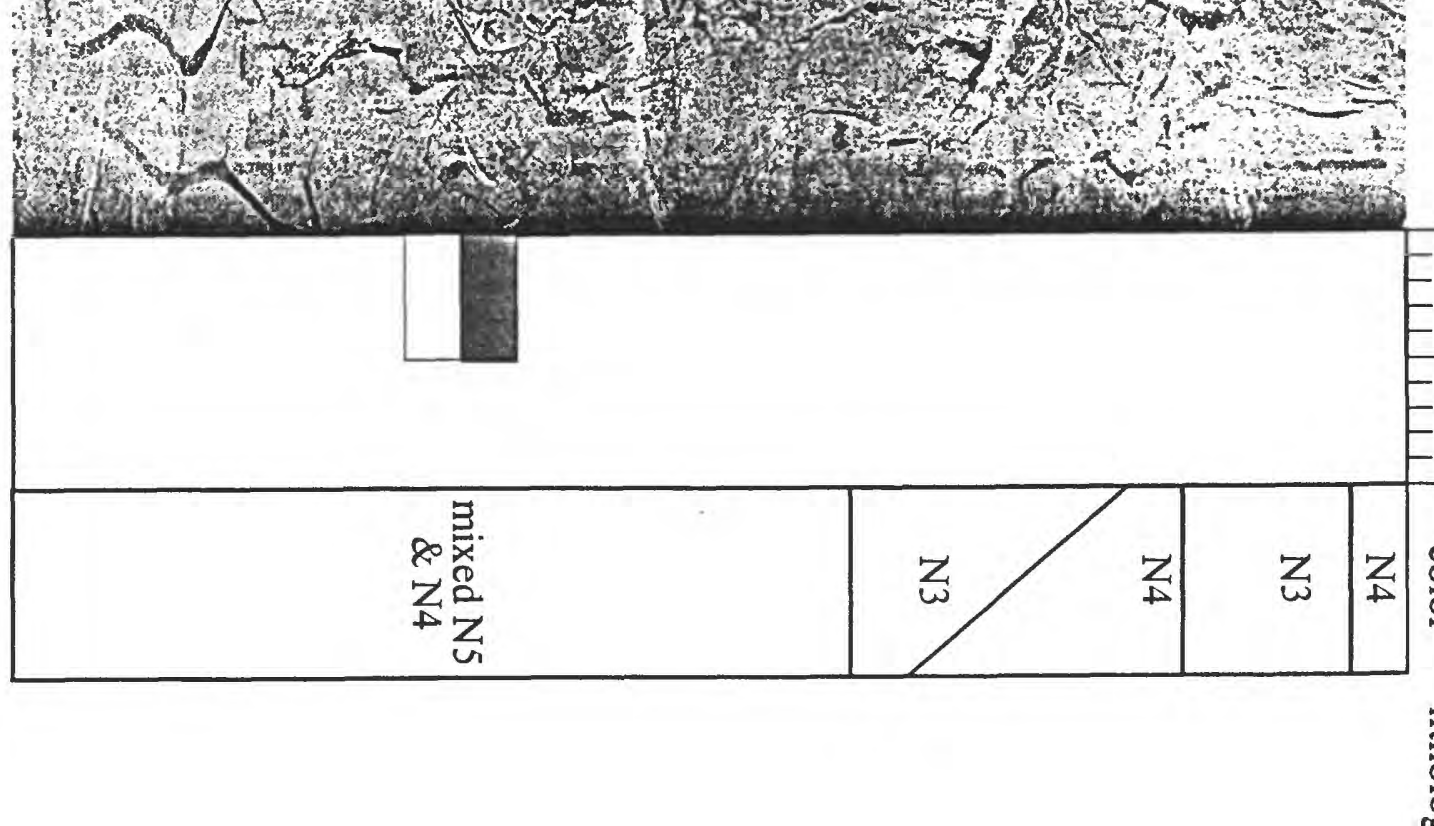

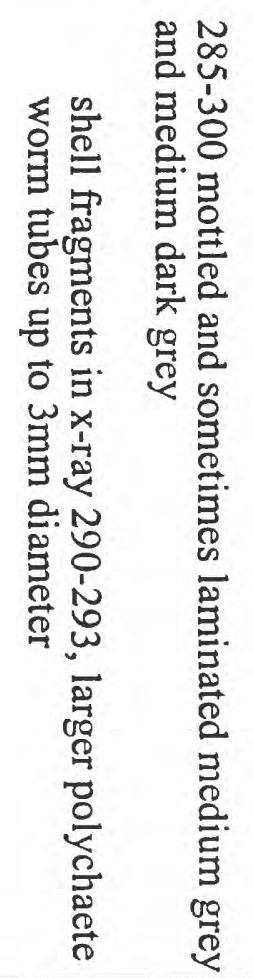

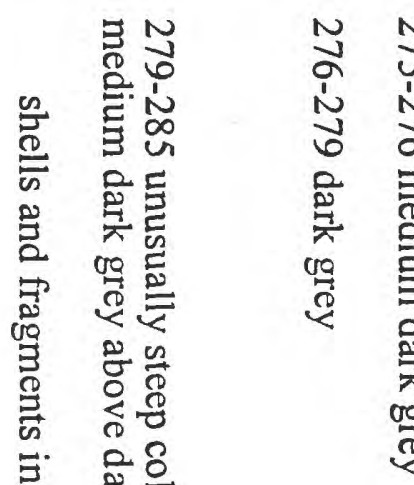

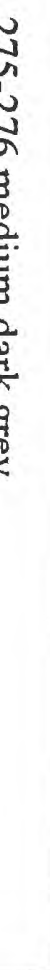

狊 


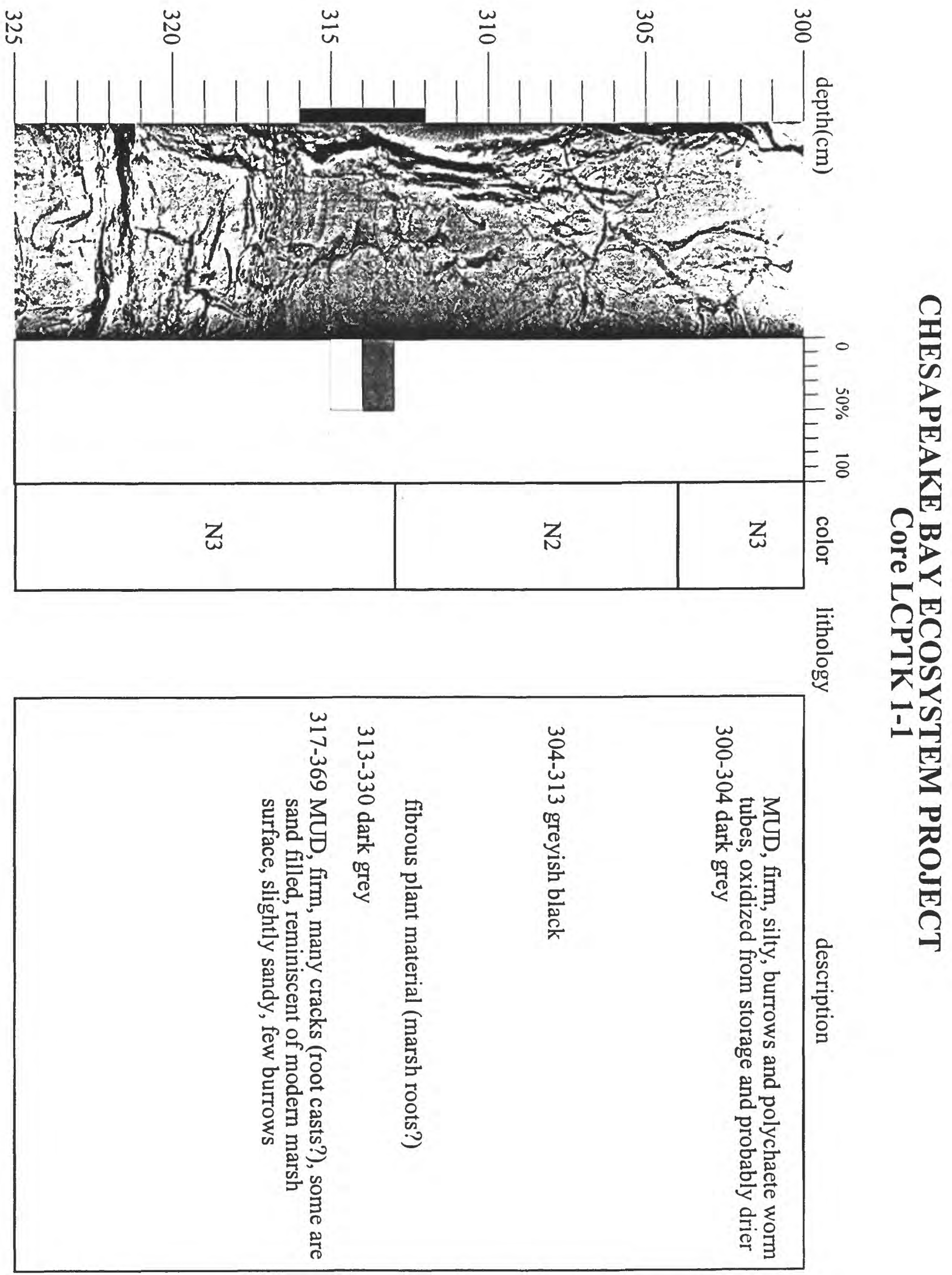




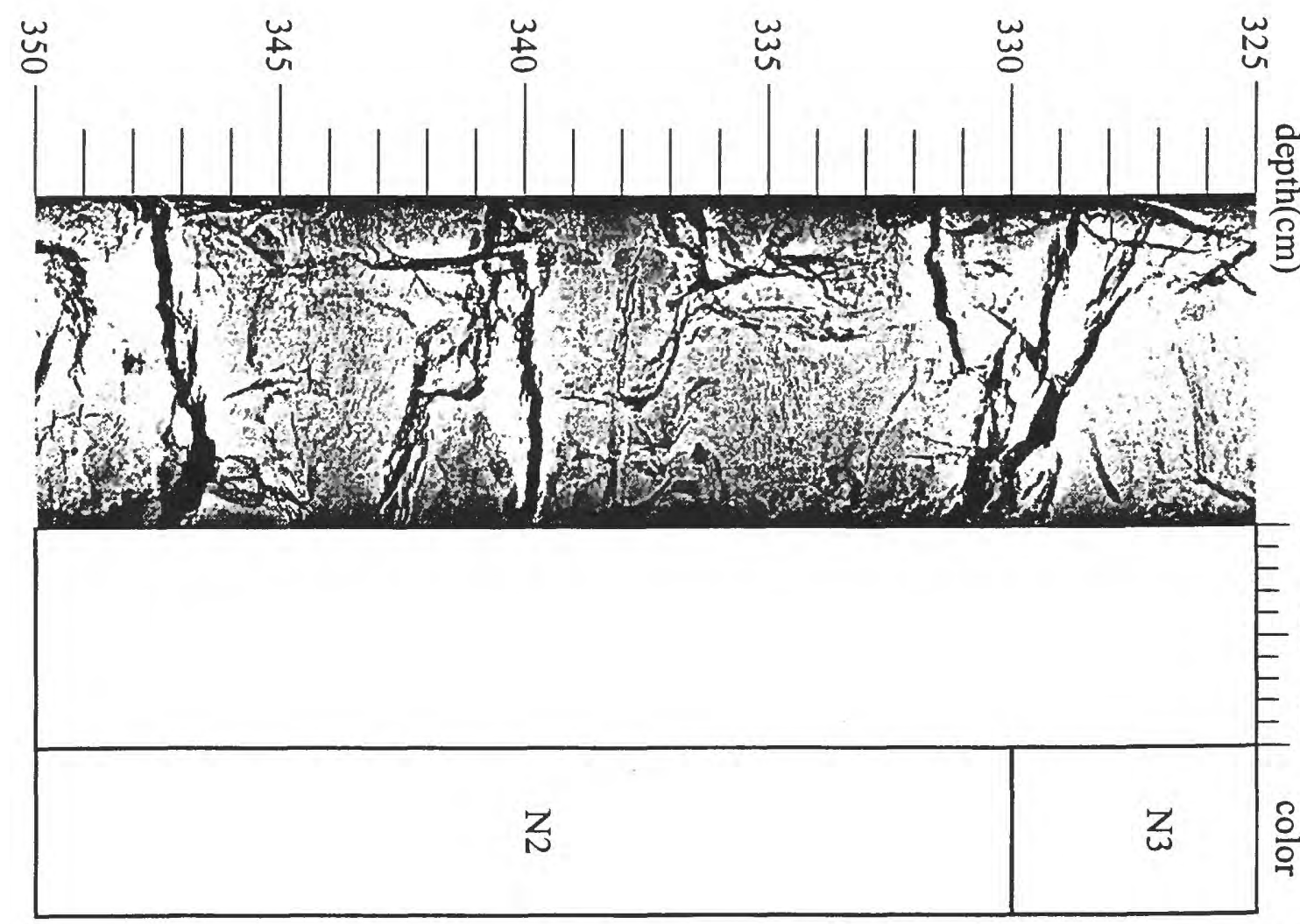

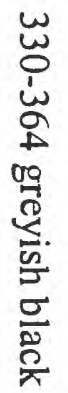

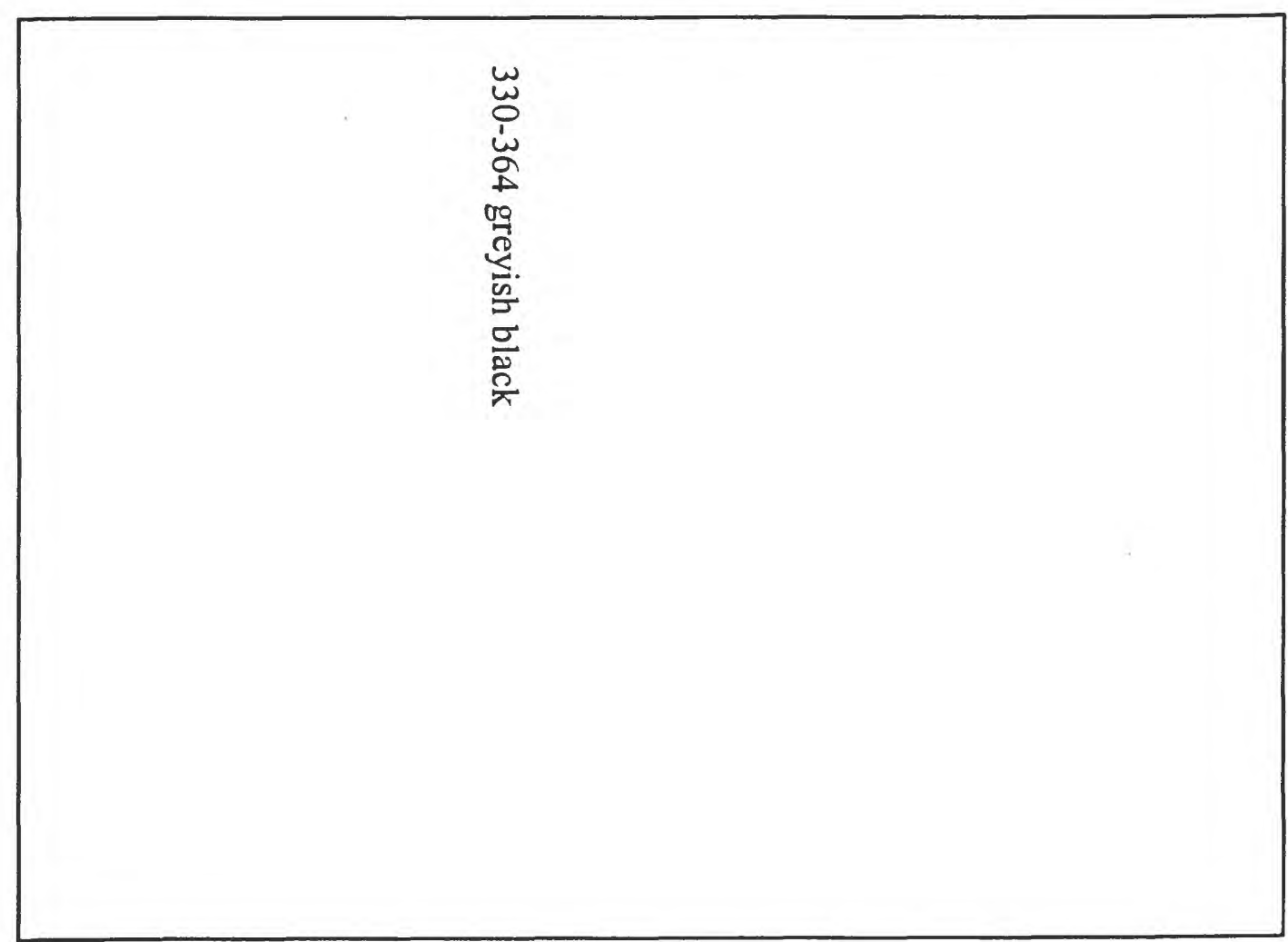



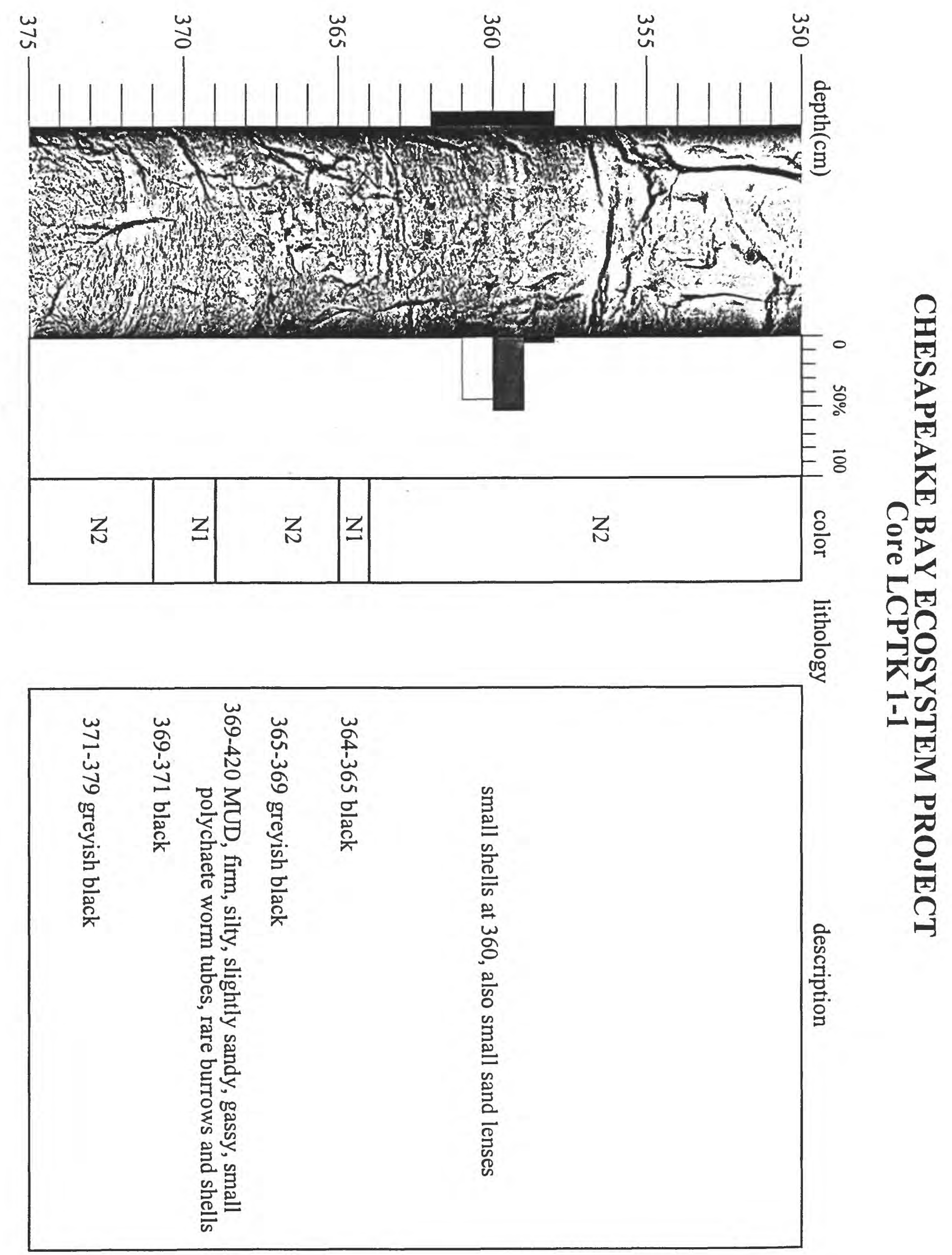

v 
8 岕 岁 岕 总 岕
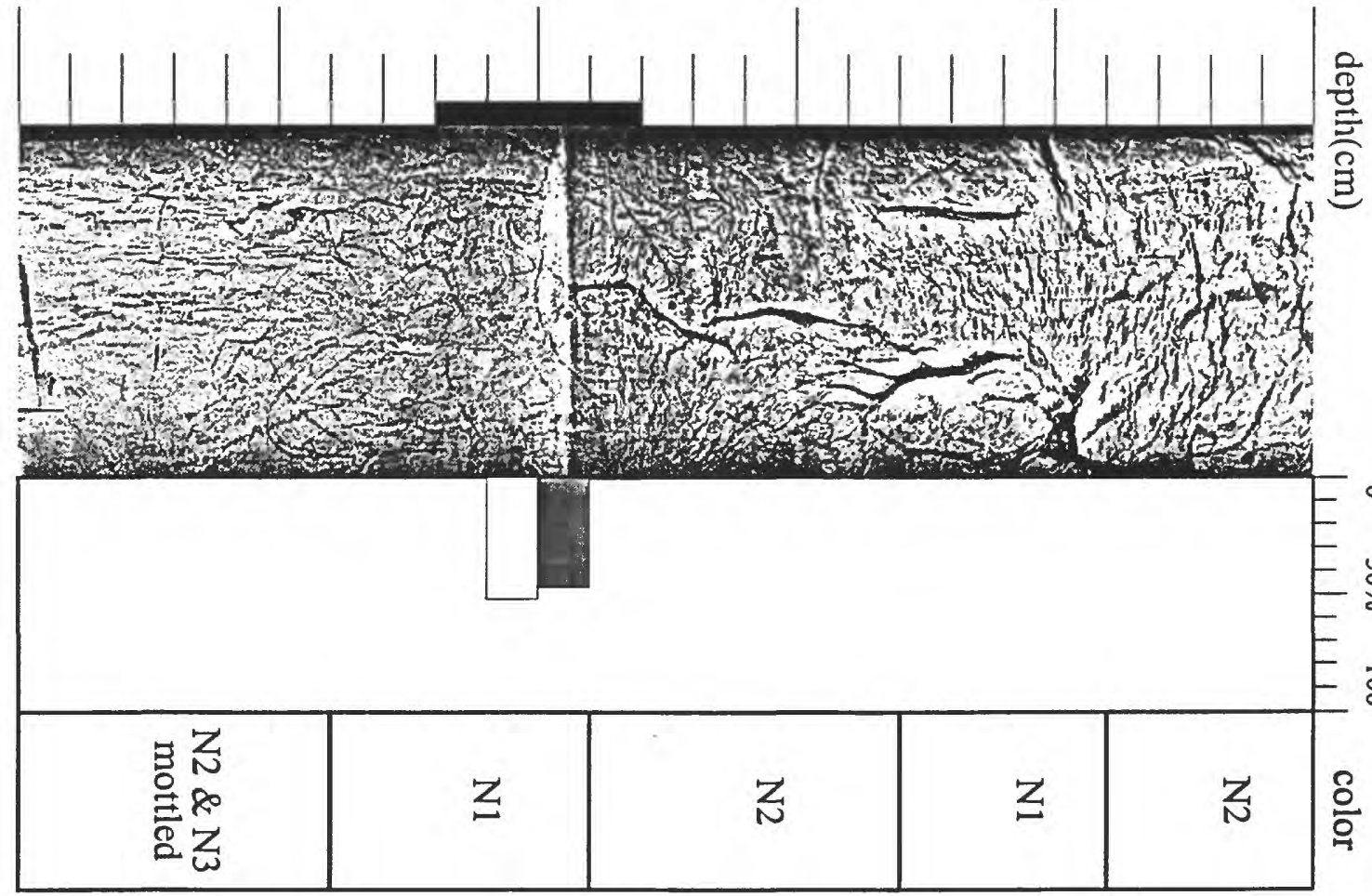

$\infty$

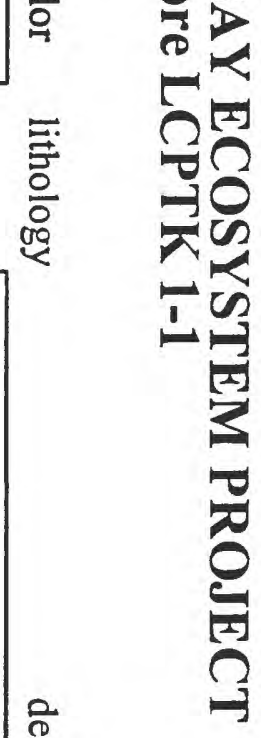




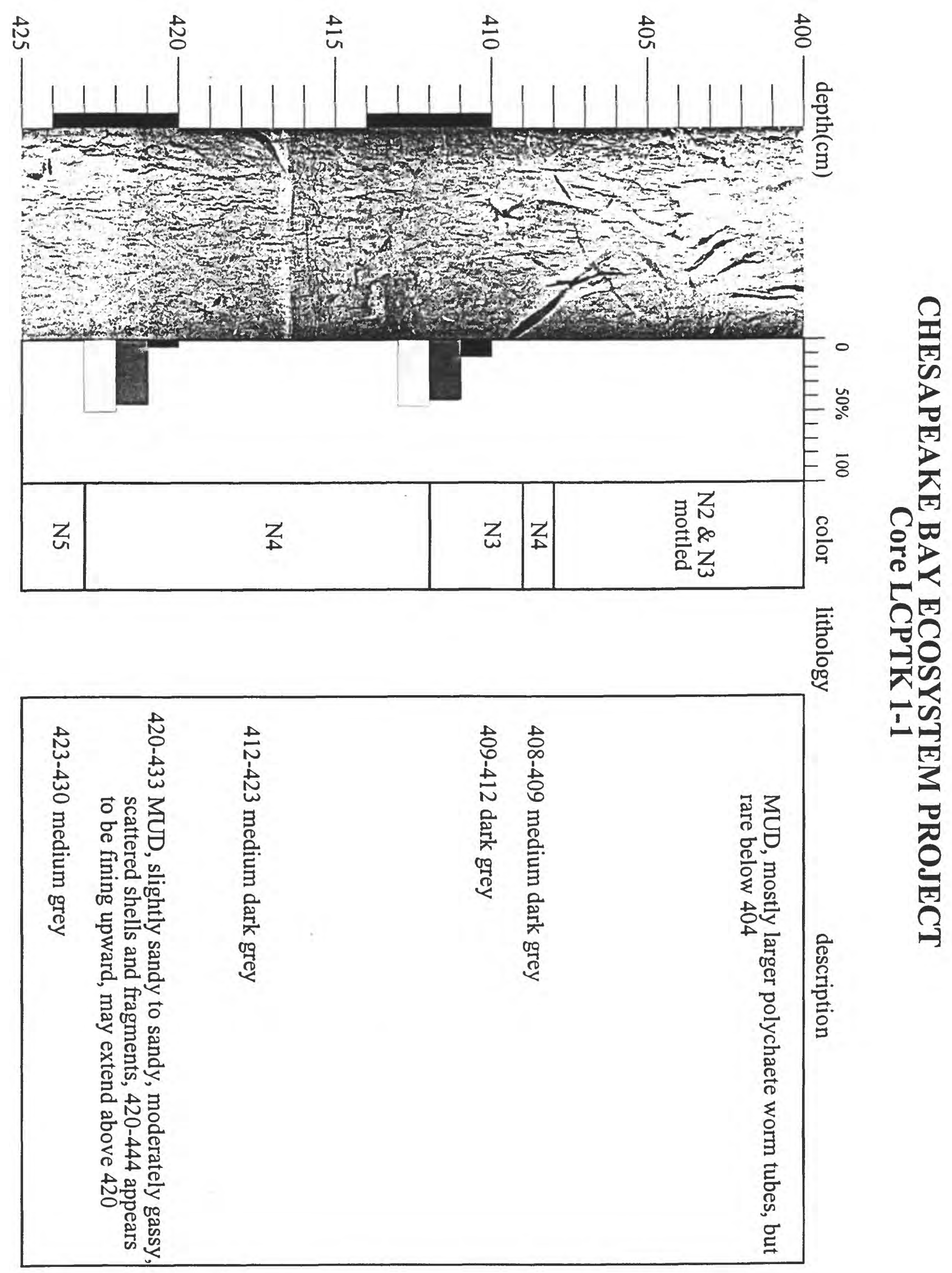



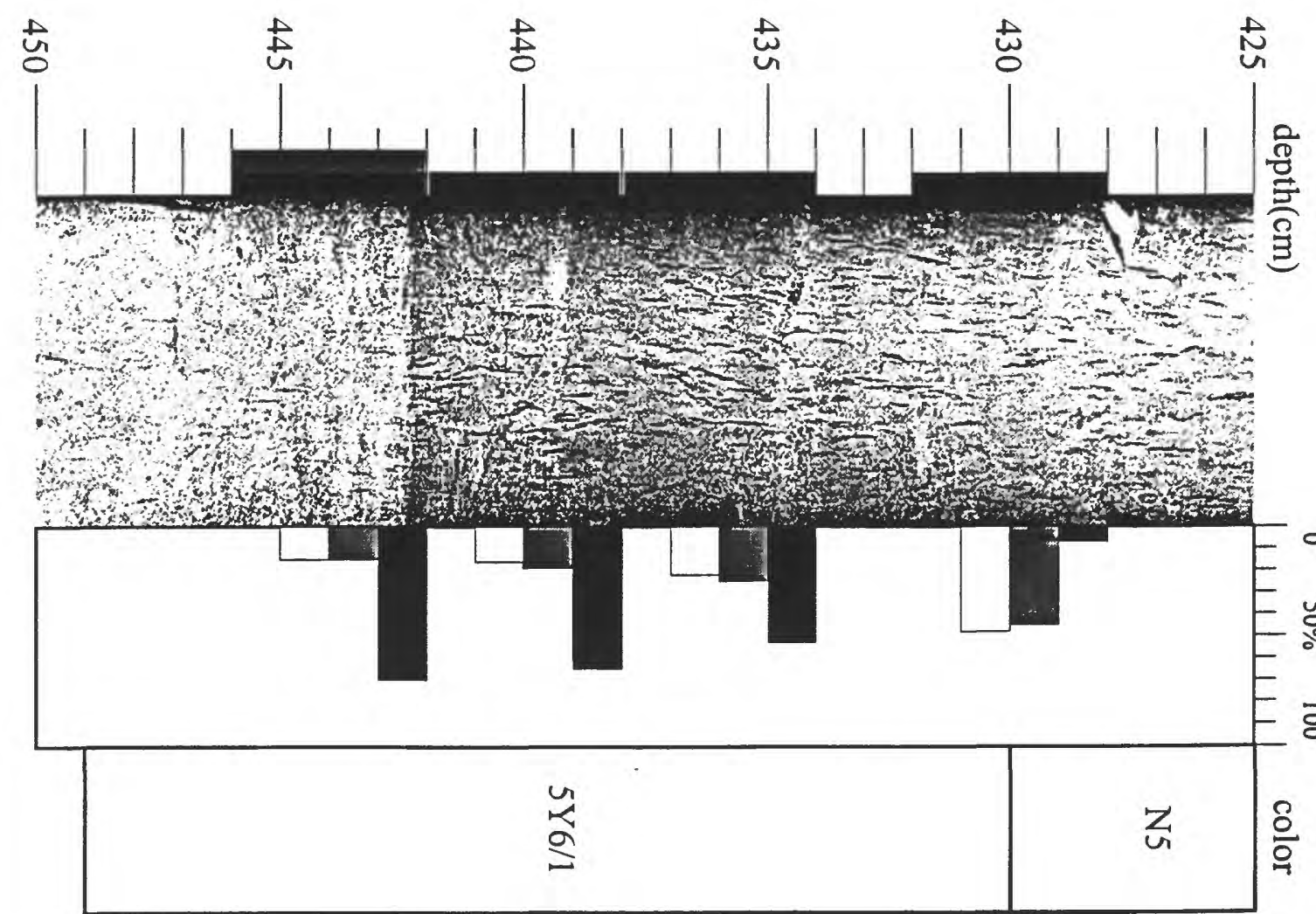

咅
-
0
0
0
3
0
0
0
0
0
0

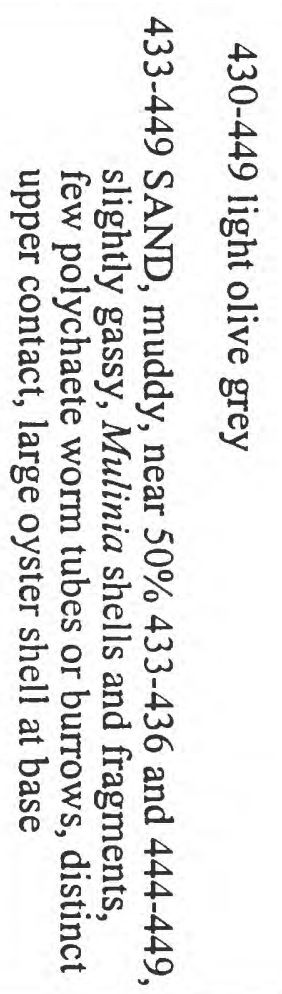

.

20 


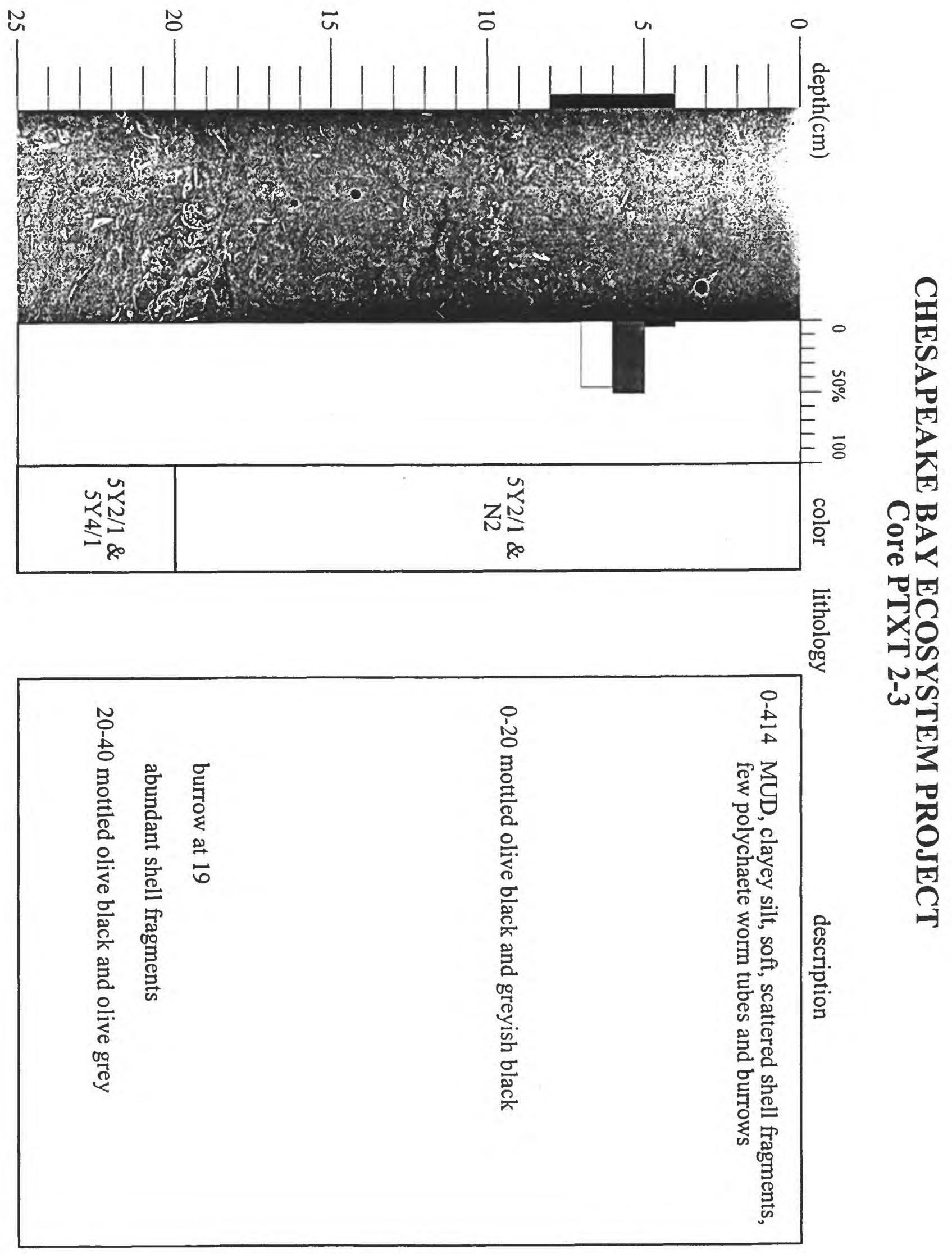




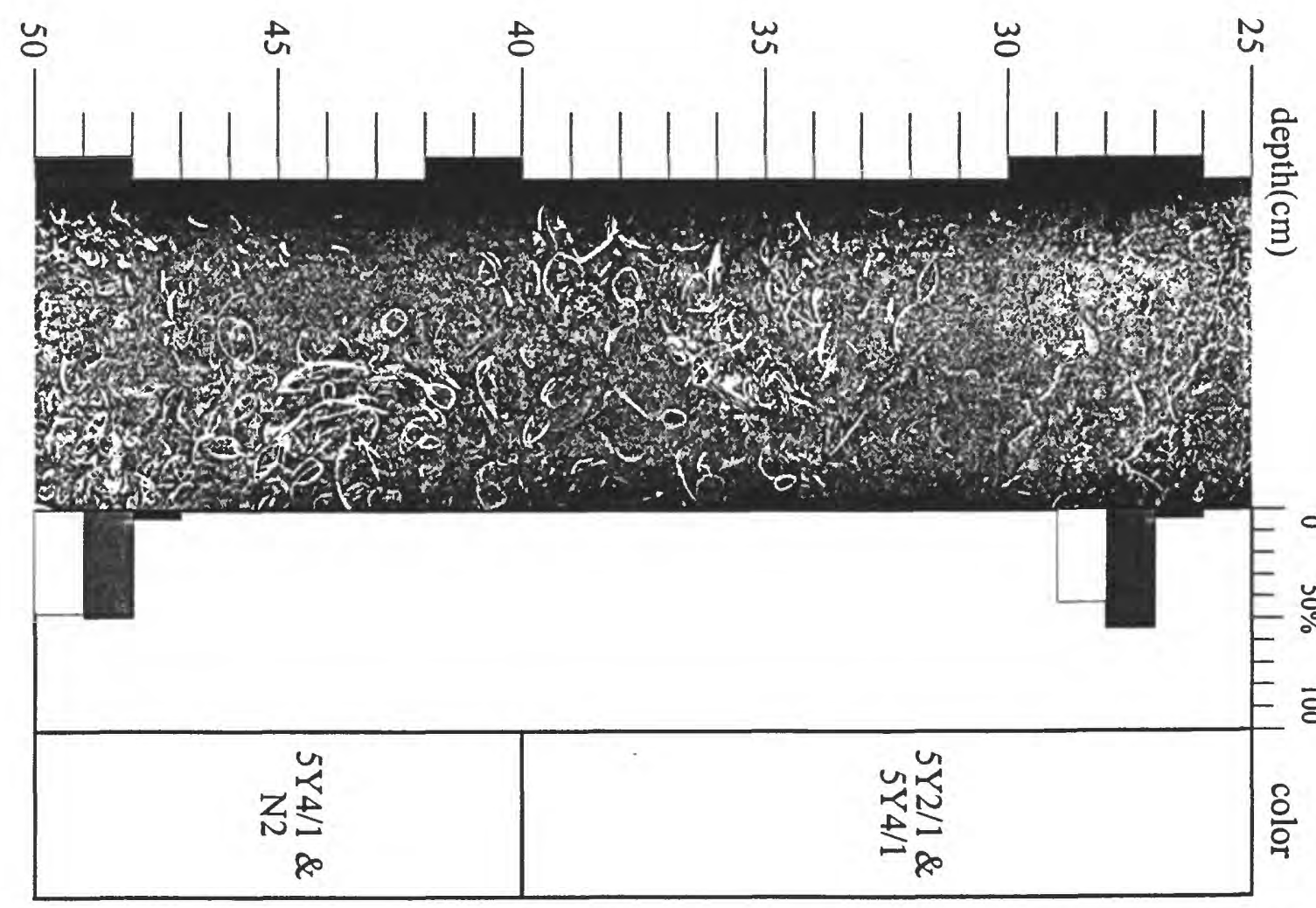

冠

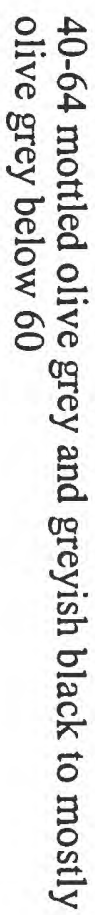

2
0
0
2
20
20
0

맘

-

$\omega$

ב3

용 

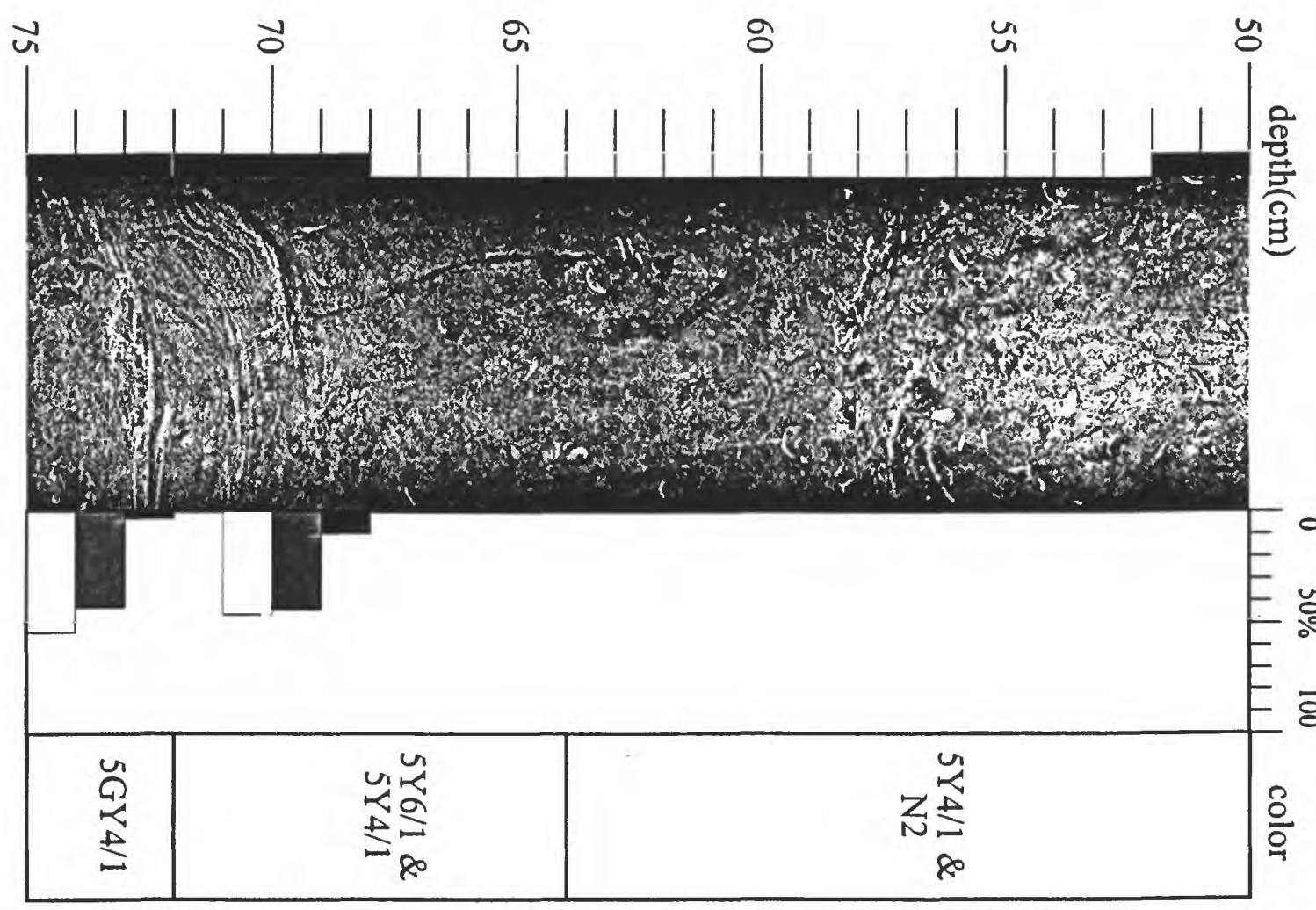

w

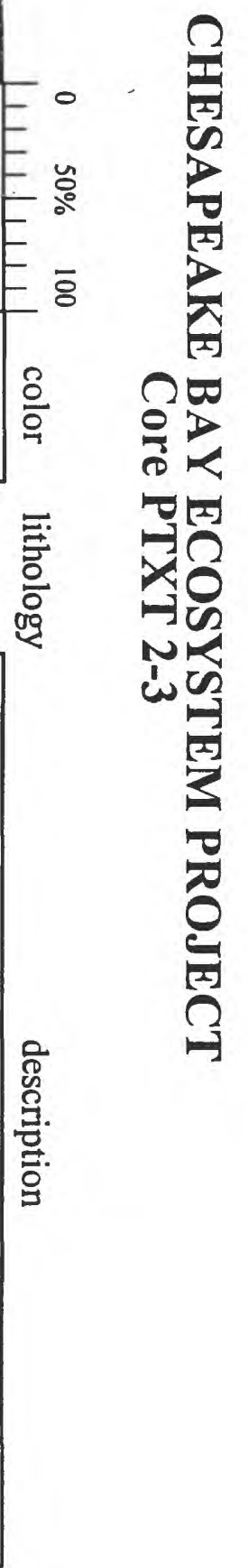



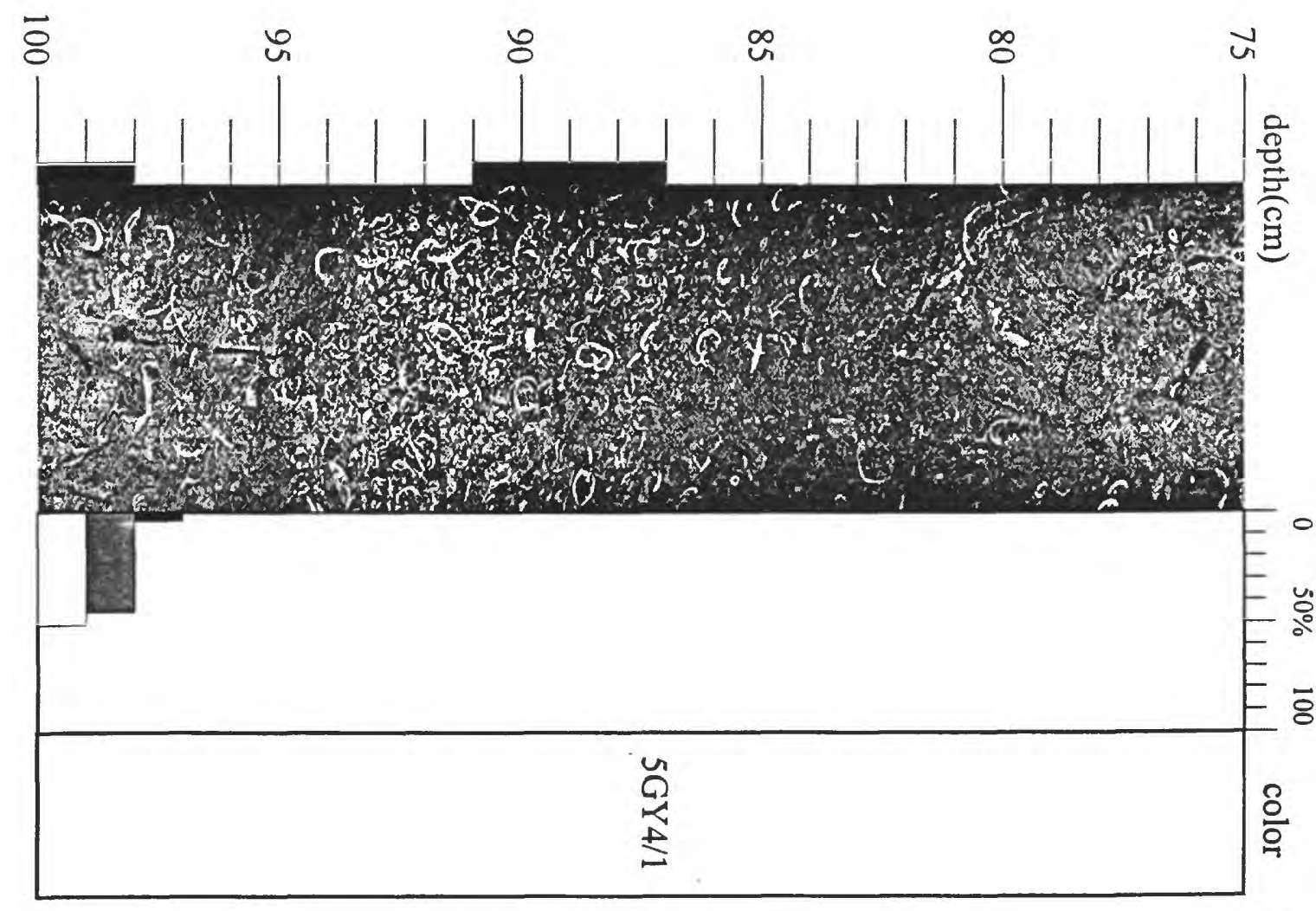

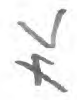

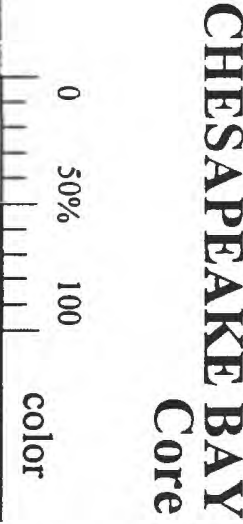

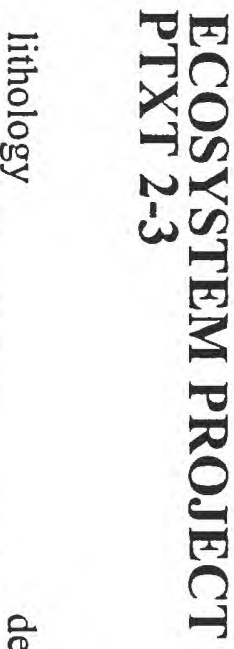



丽号

芑芯

느응

羊

实哭

号

a

넝

뭉

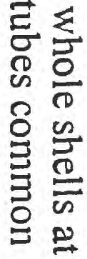



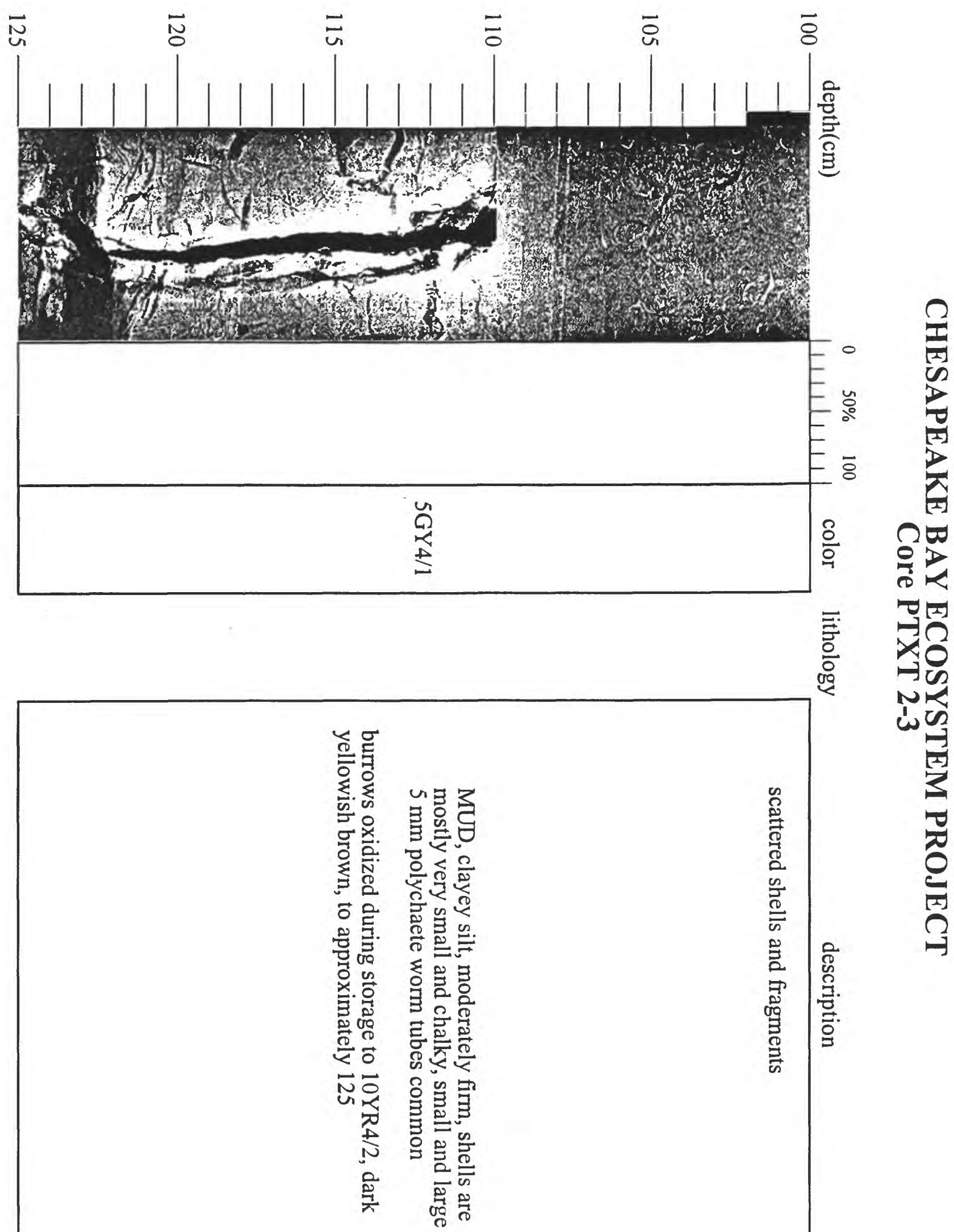


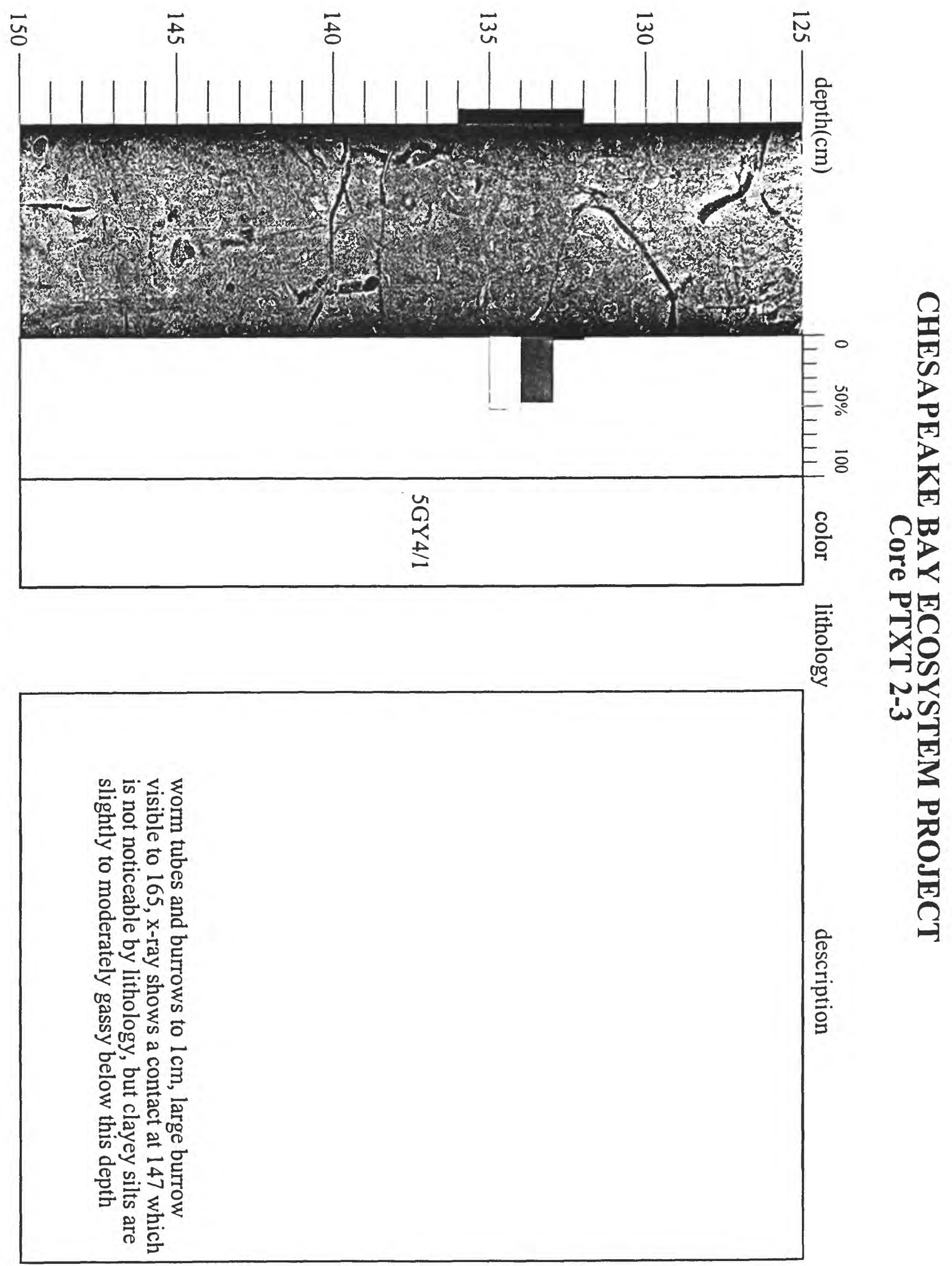



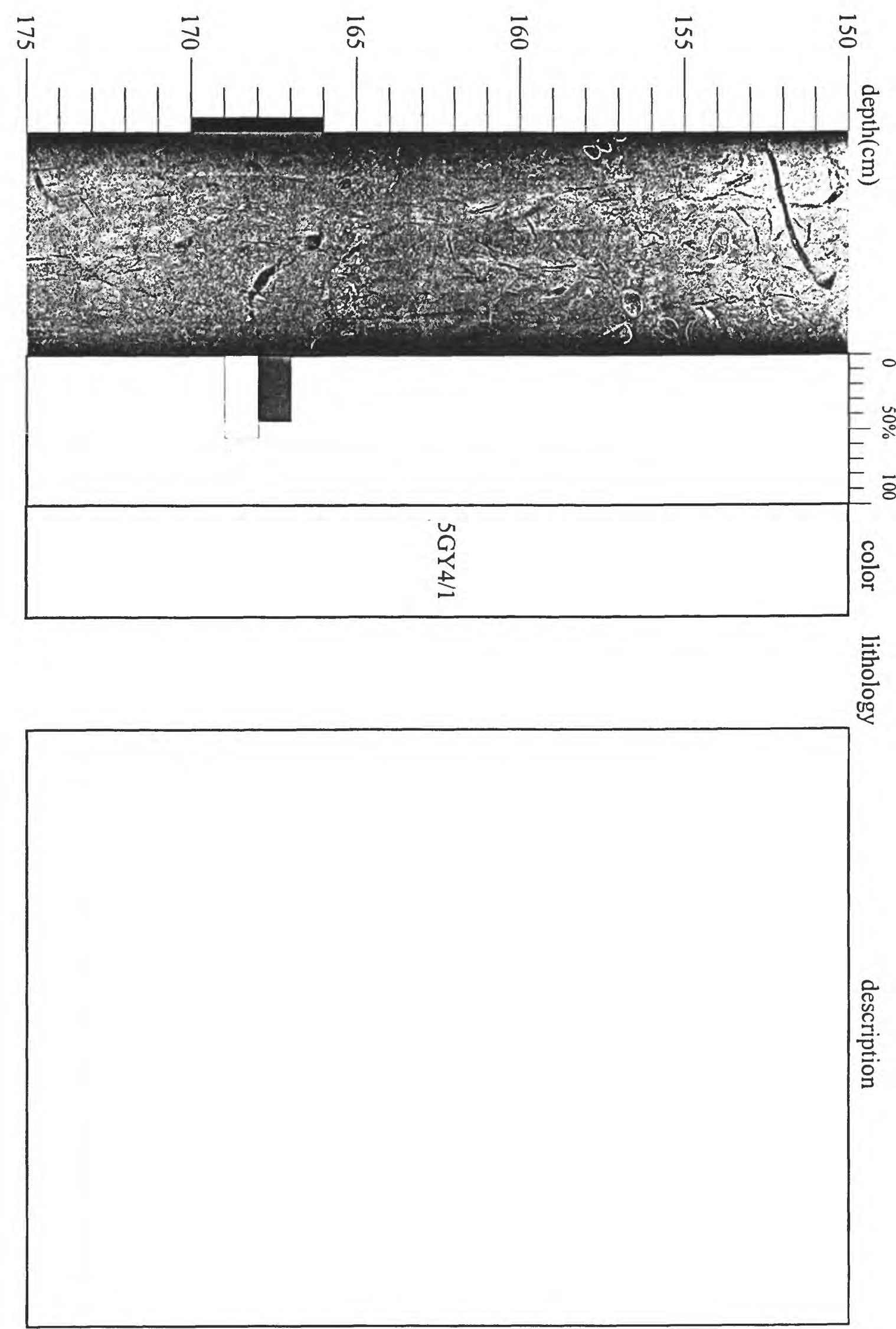


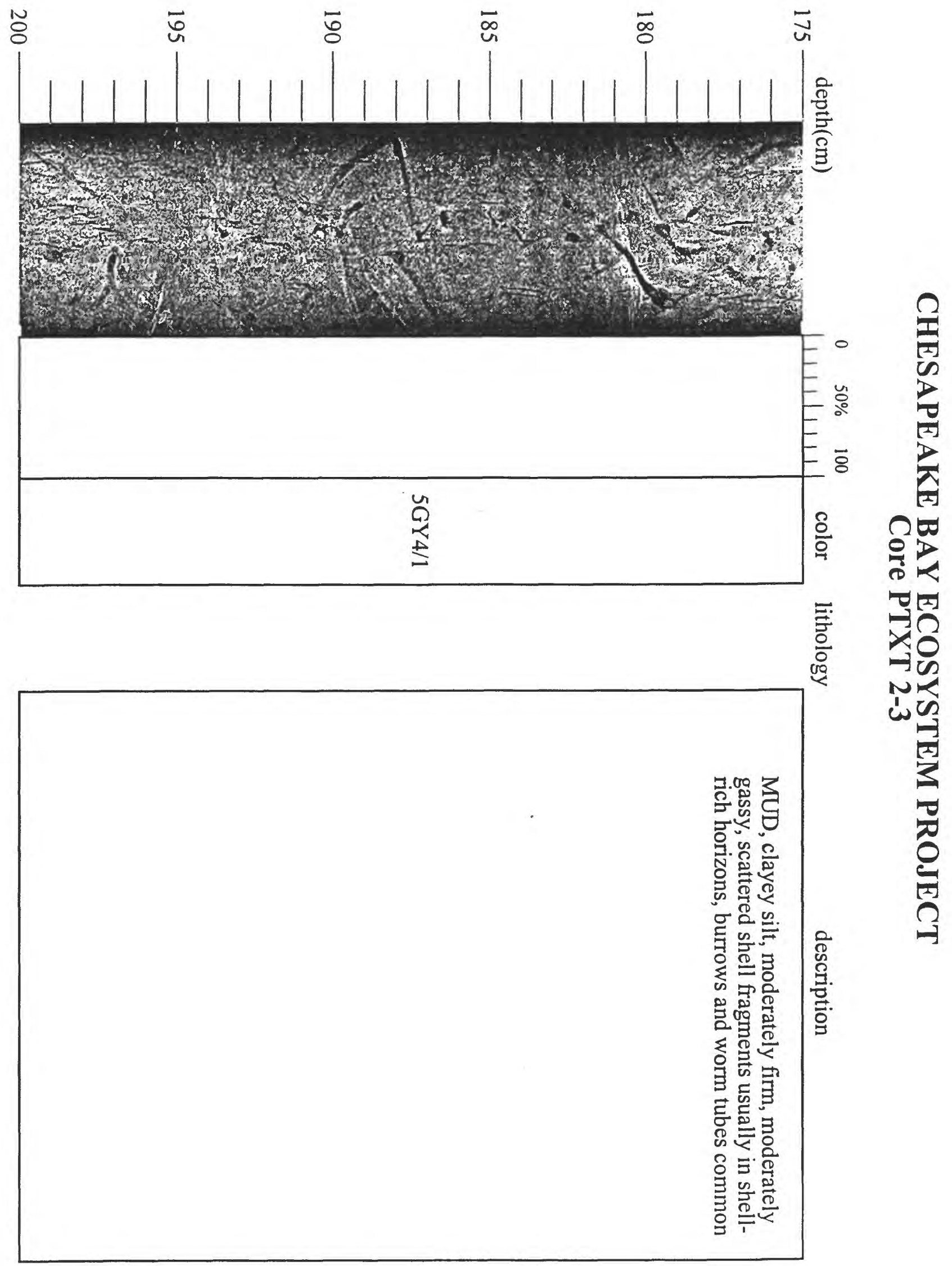




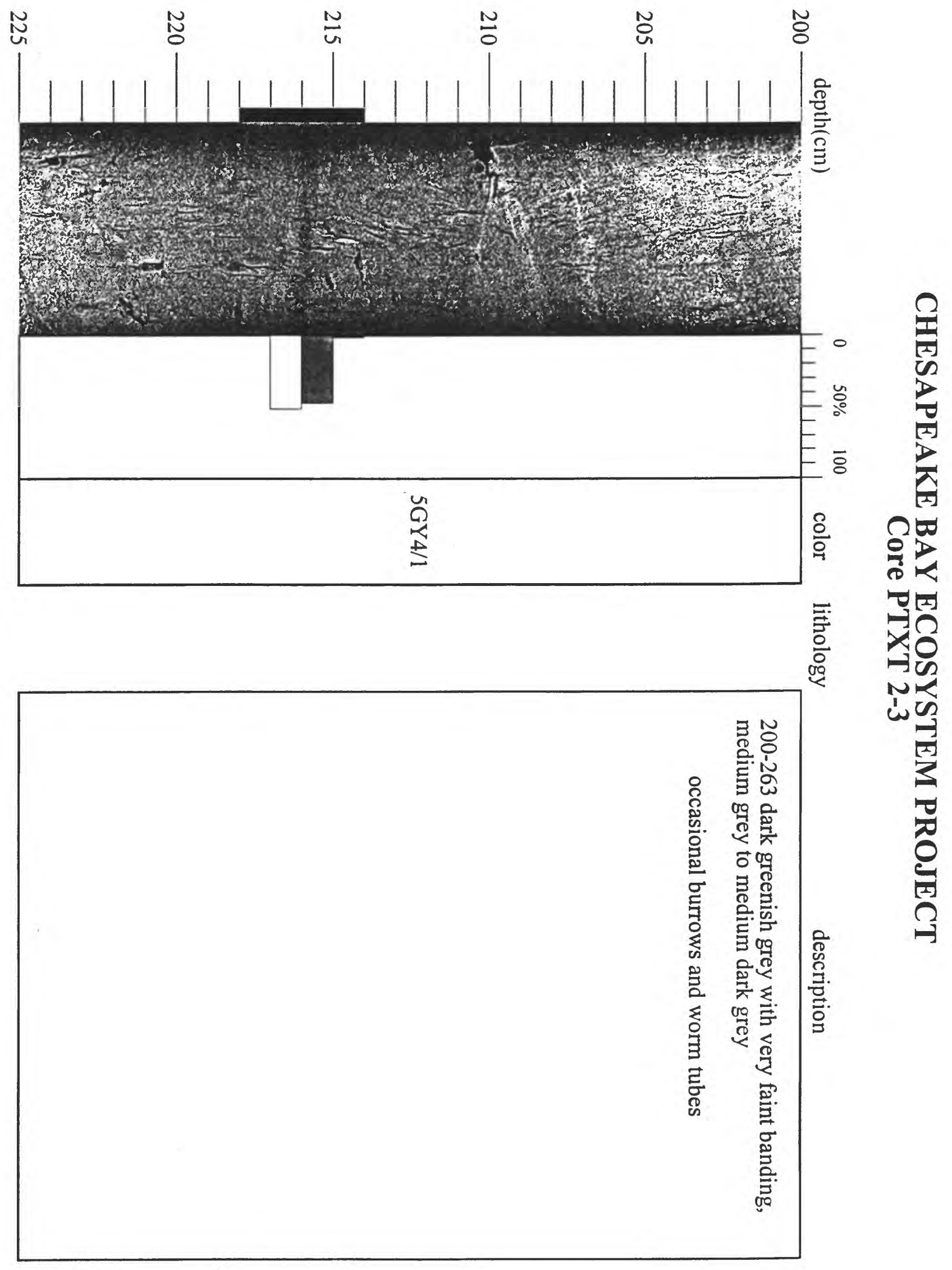



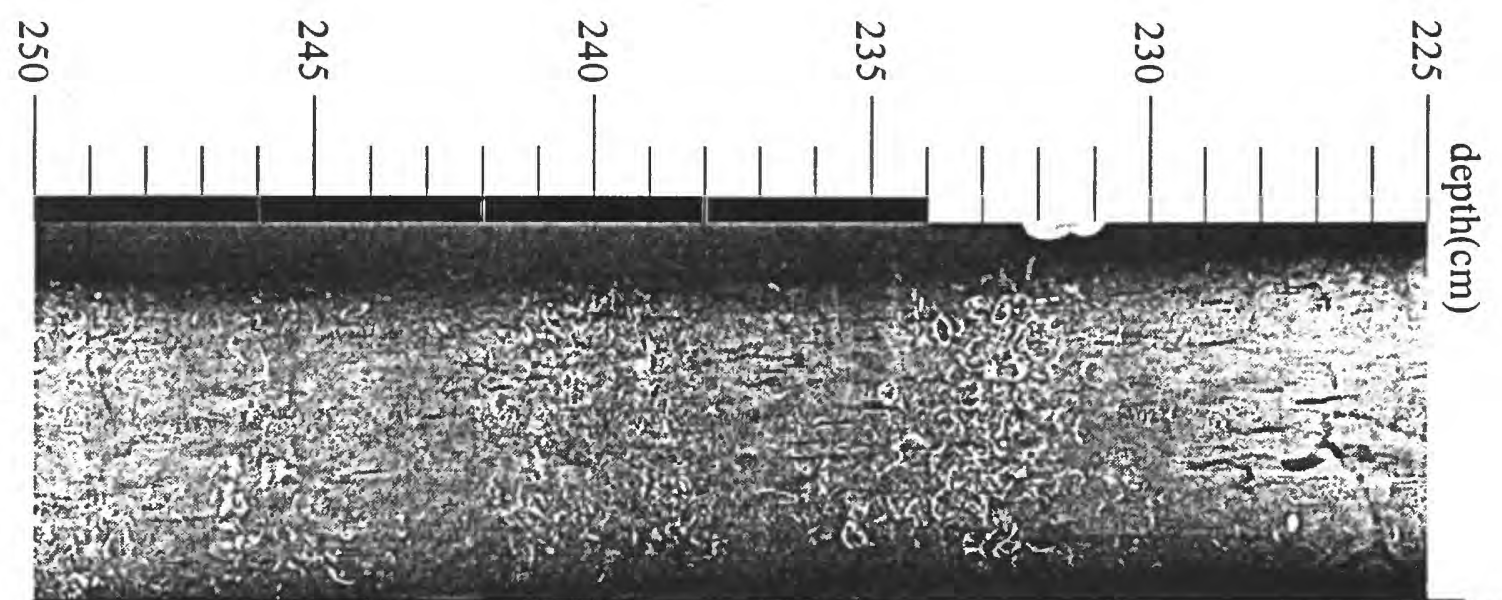

$\infty$

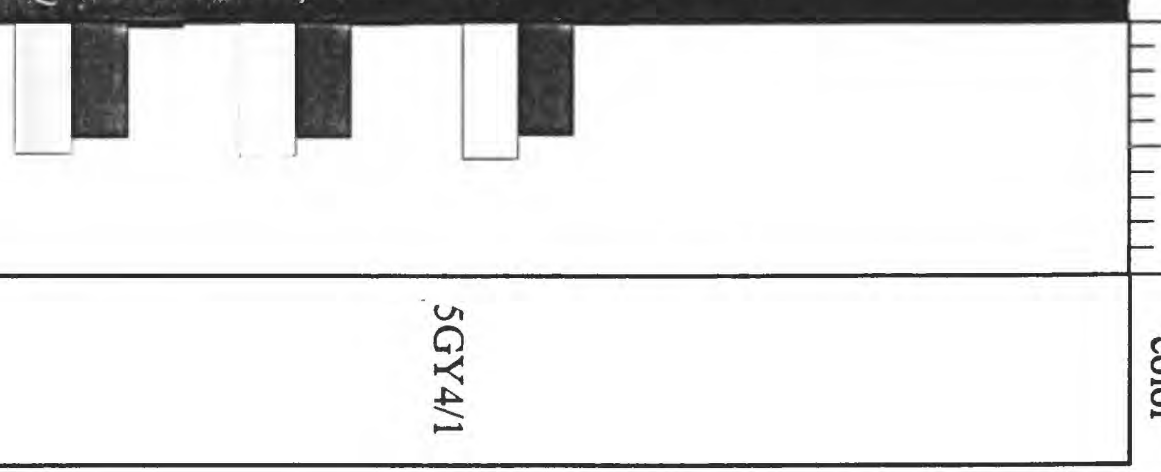

$=0$

壱

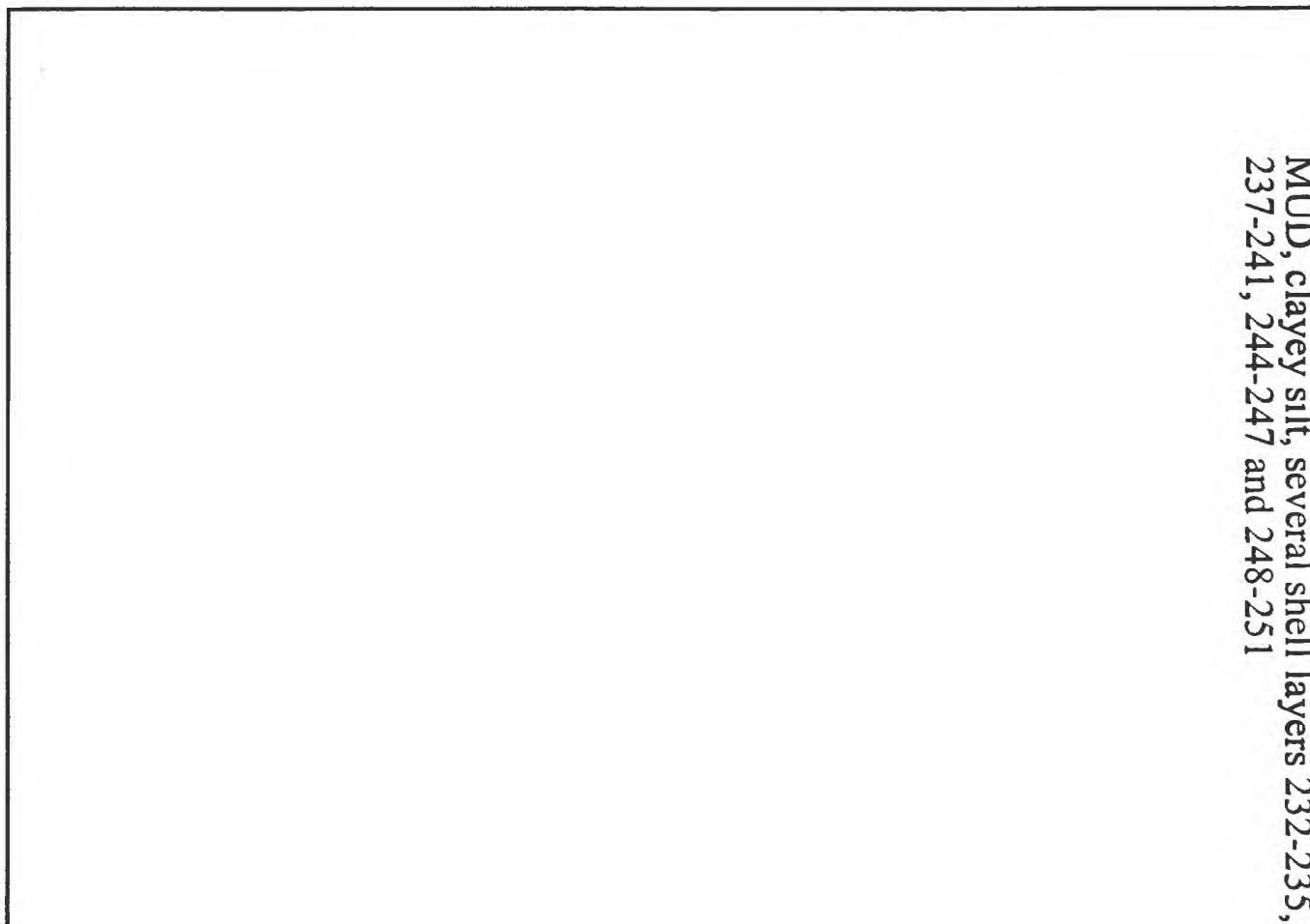




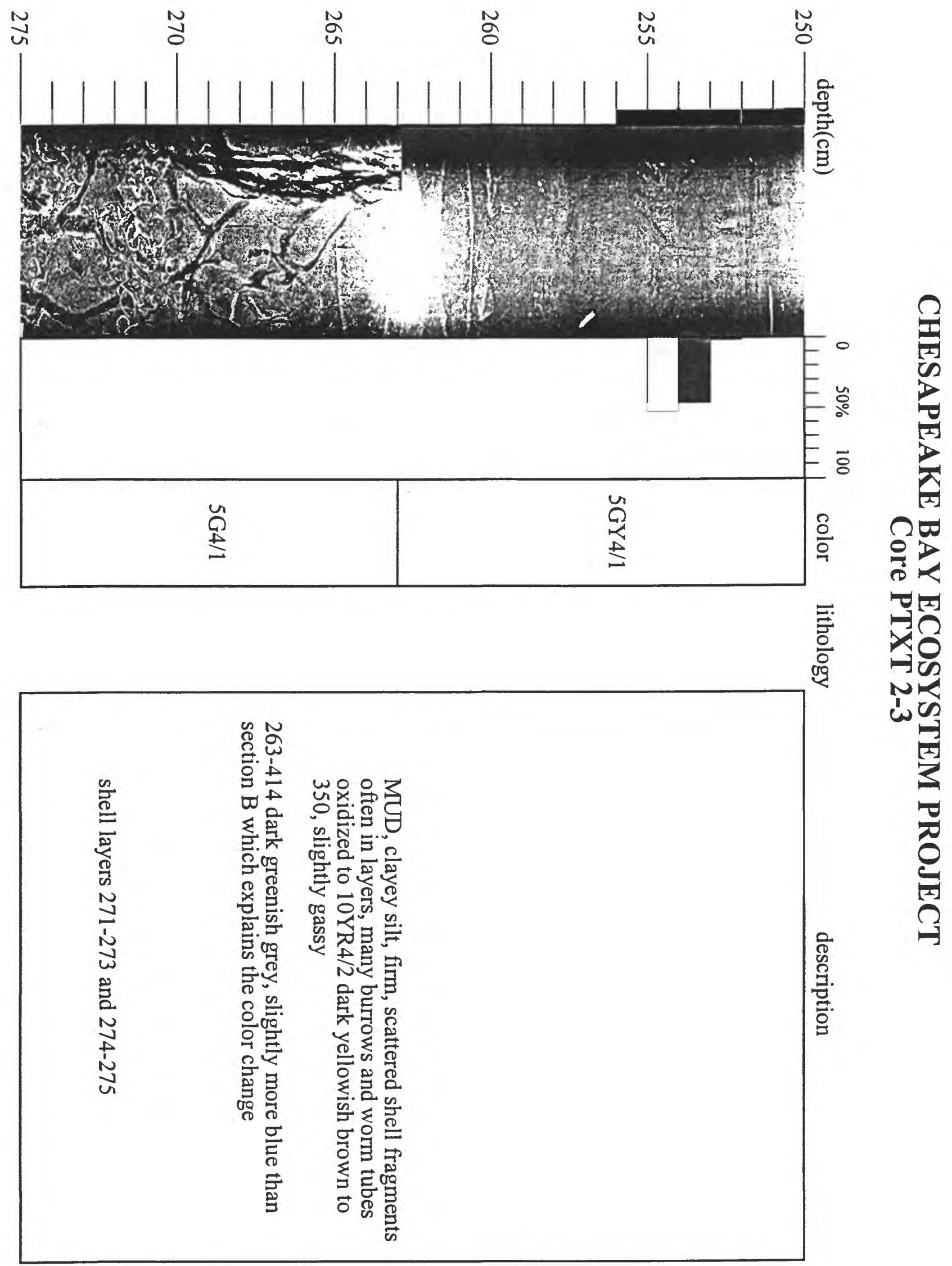




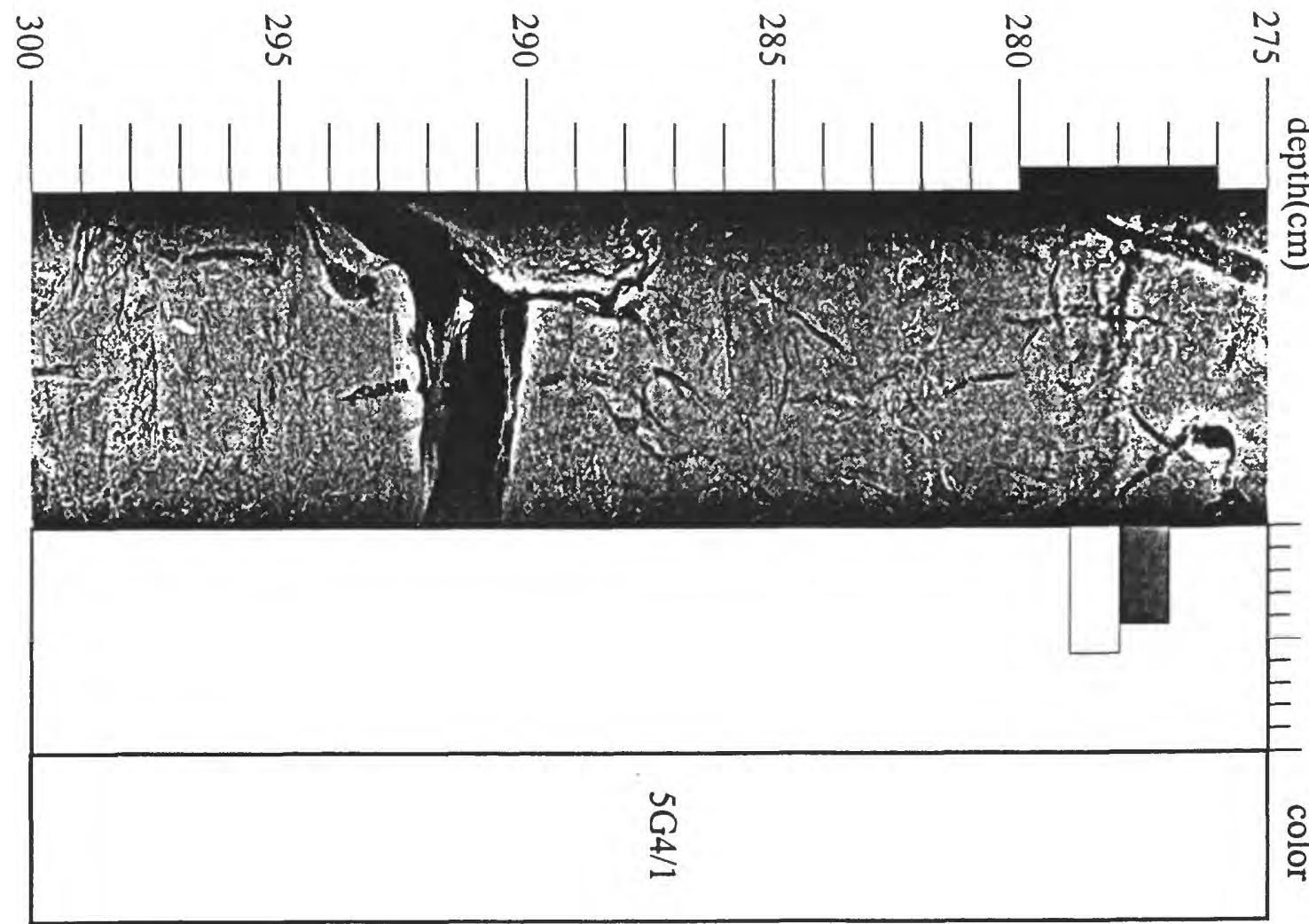

U

$=0$

豆

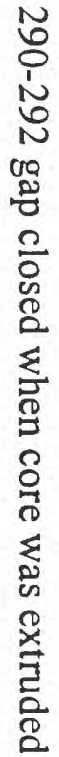

苛. 


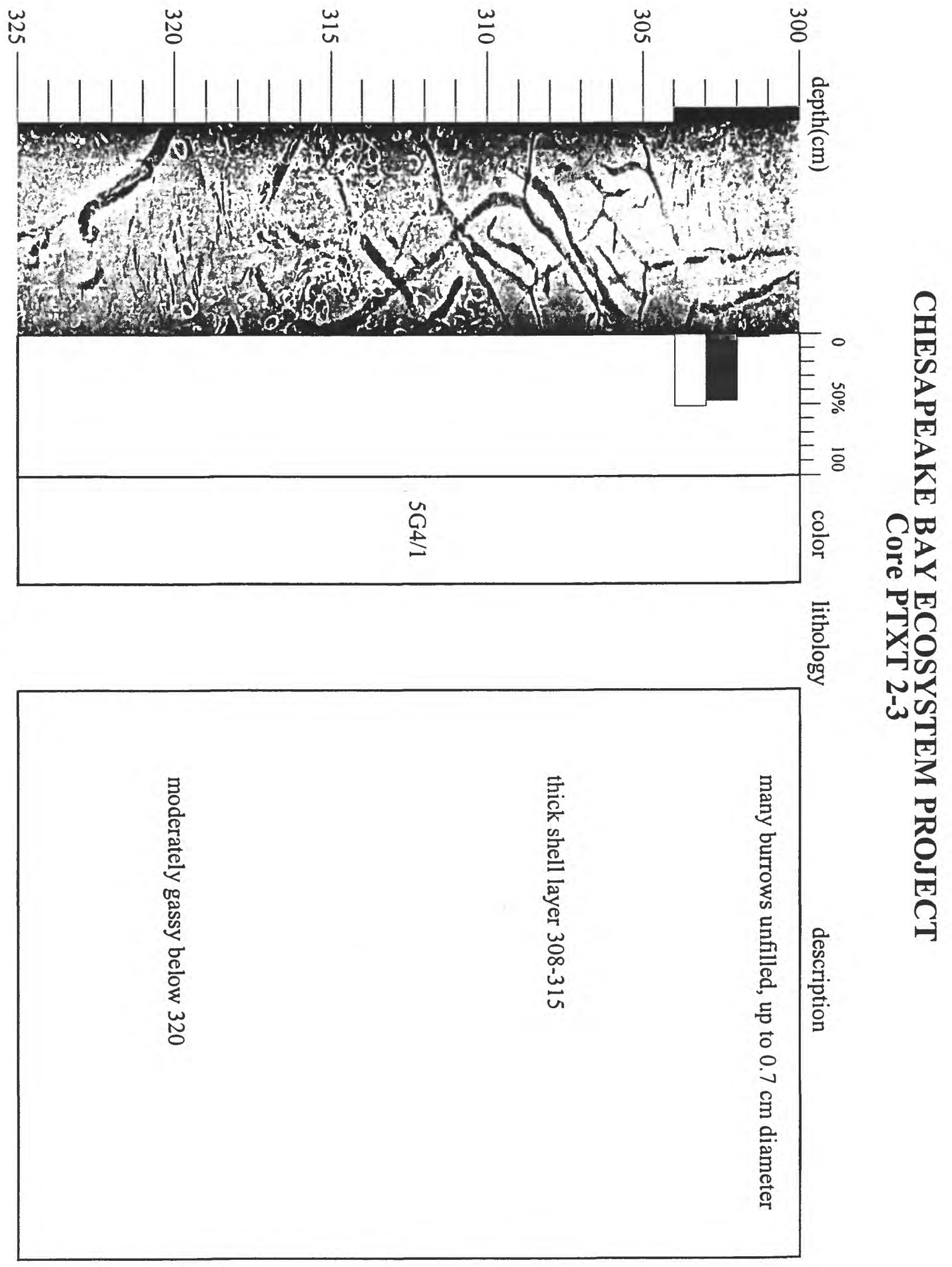




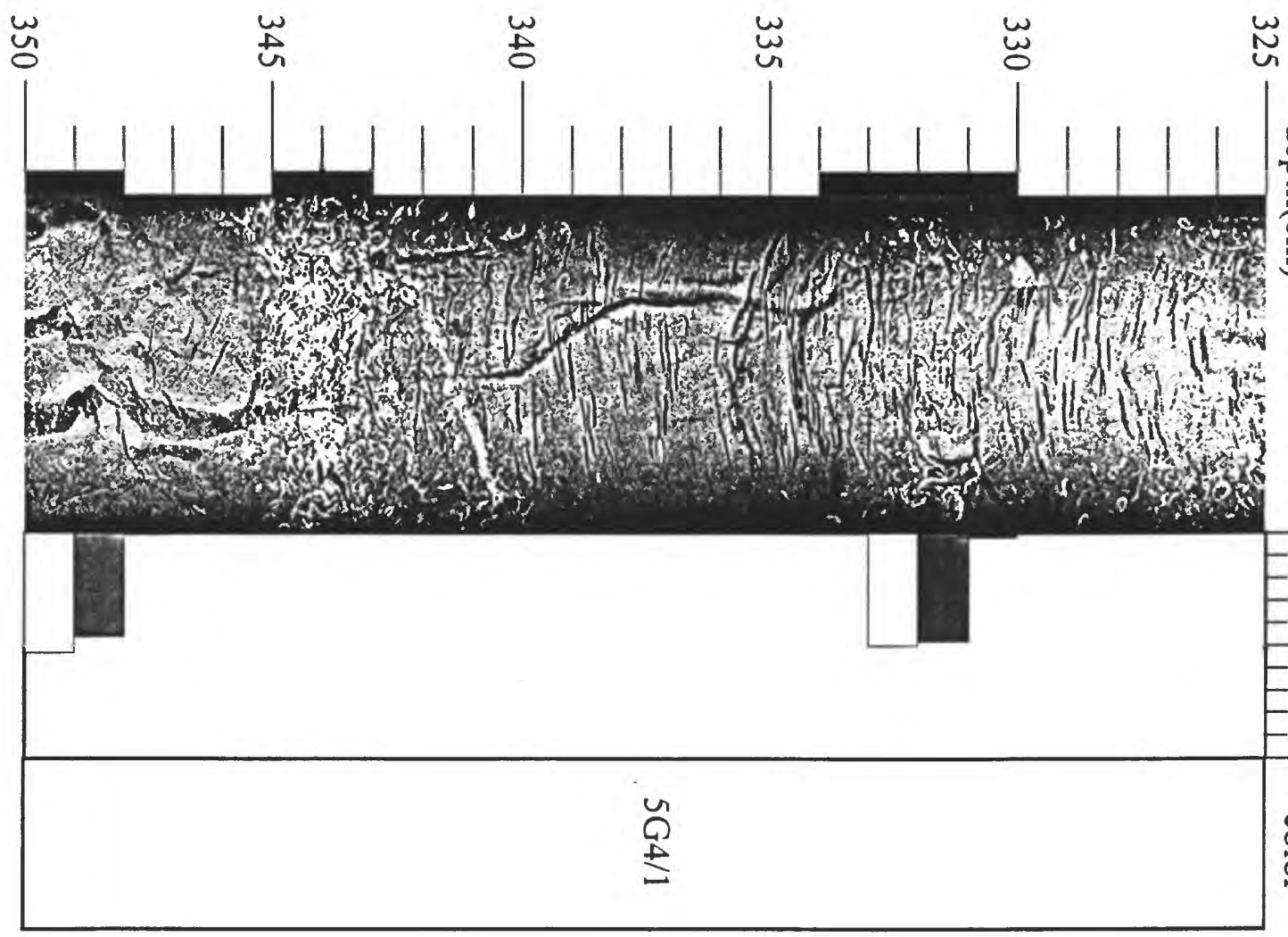

章

$x$
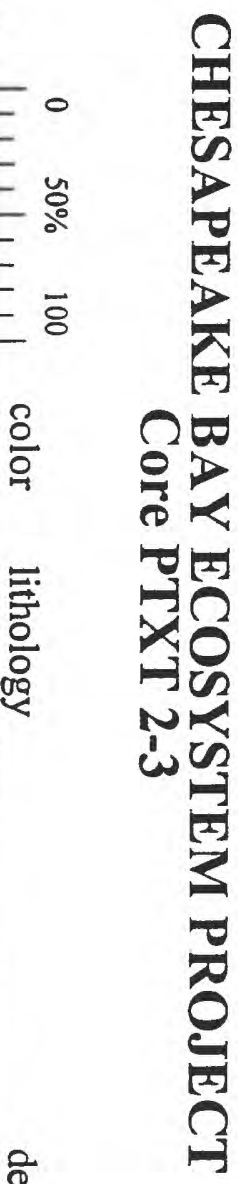

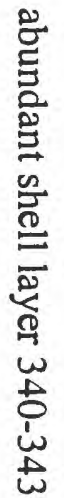

ก

. 


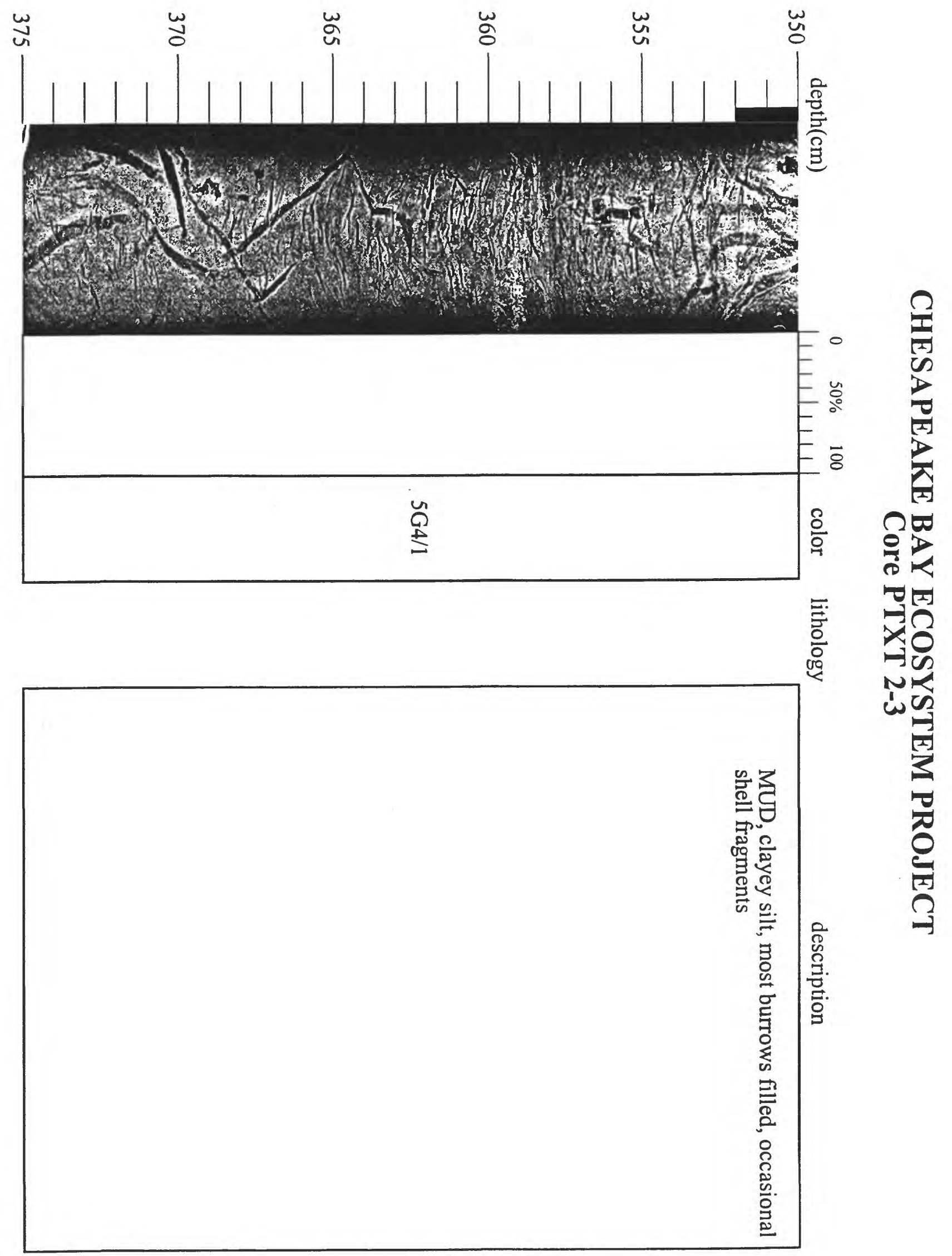




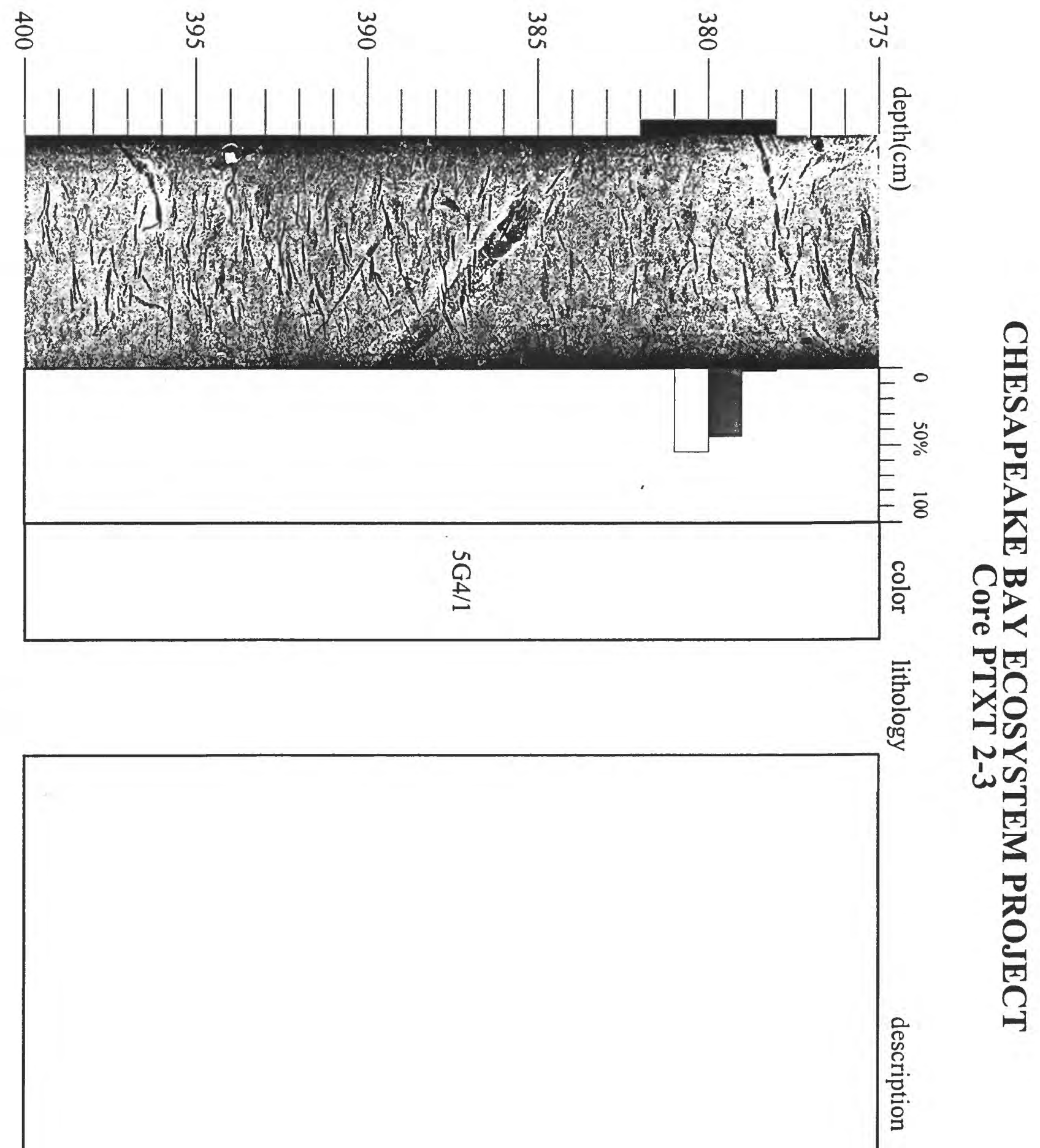




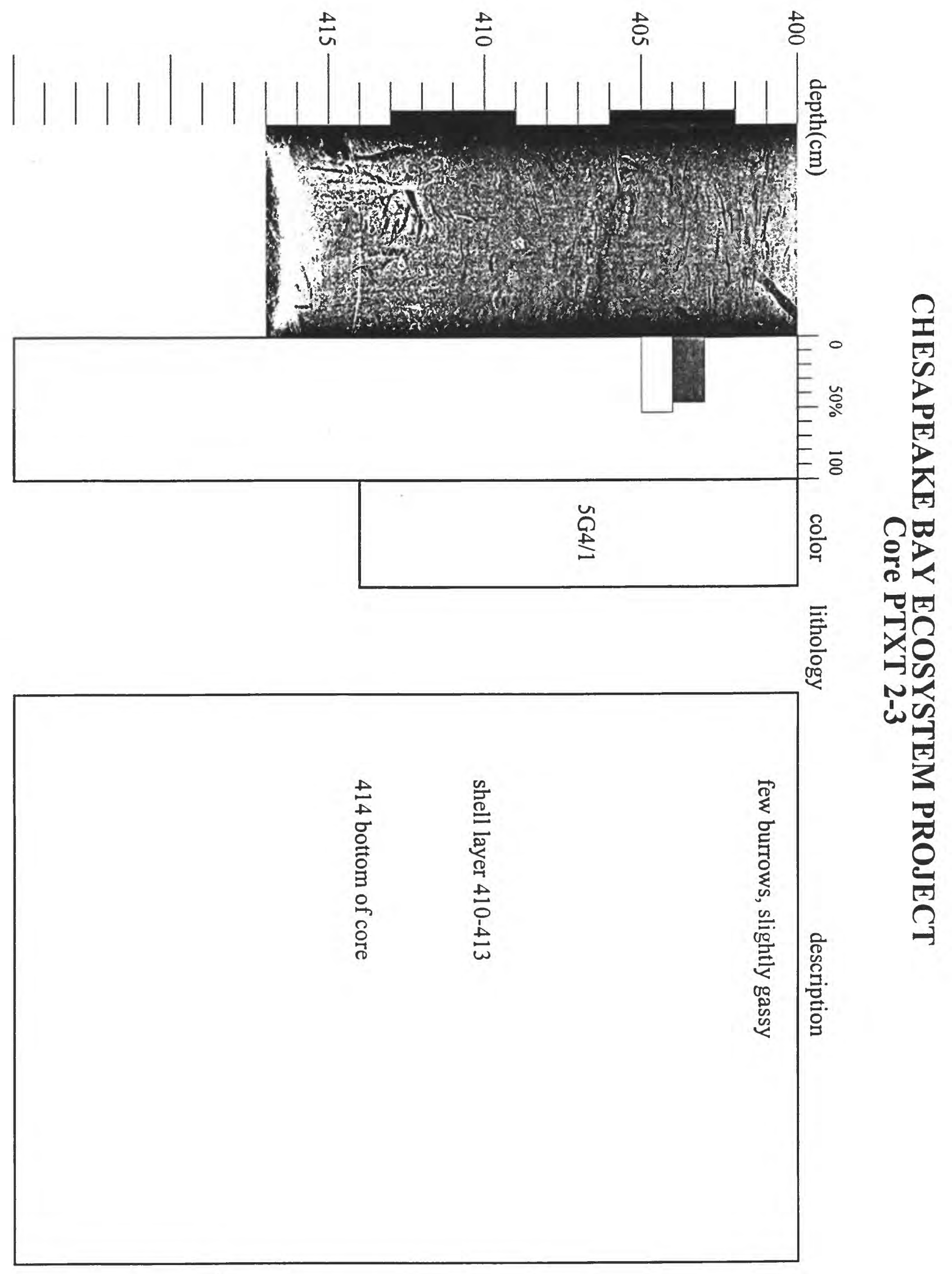



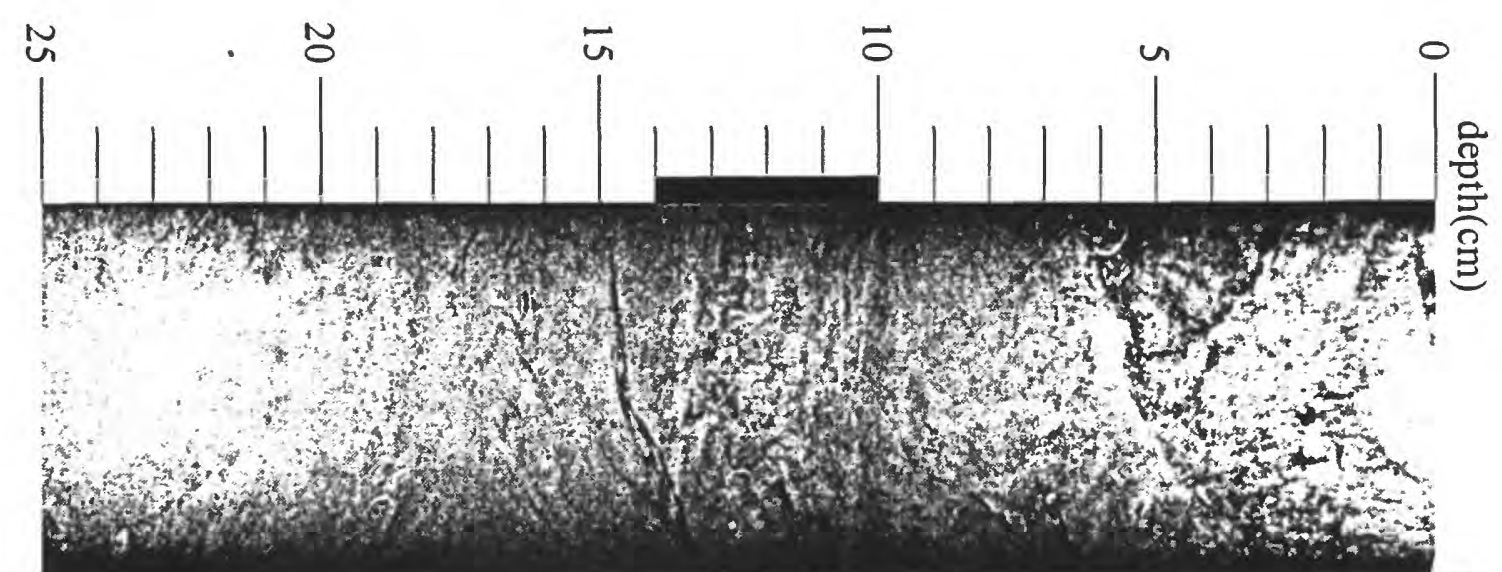

$\infty$
$\infty$

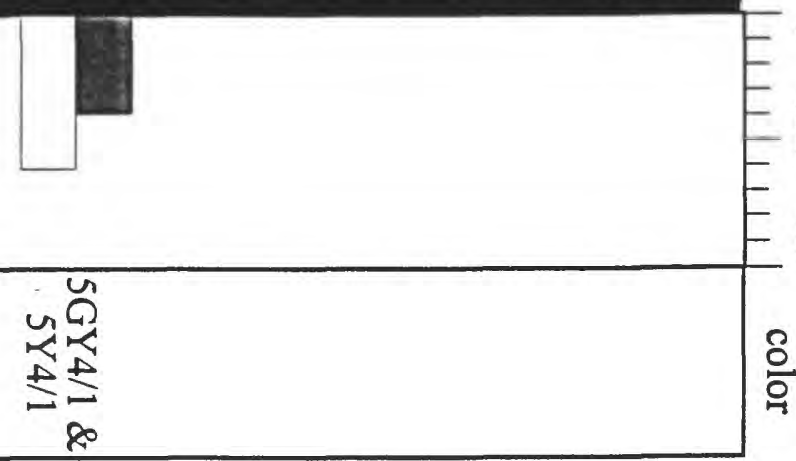

$=$ - $=0$

- T[x]

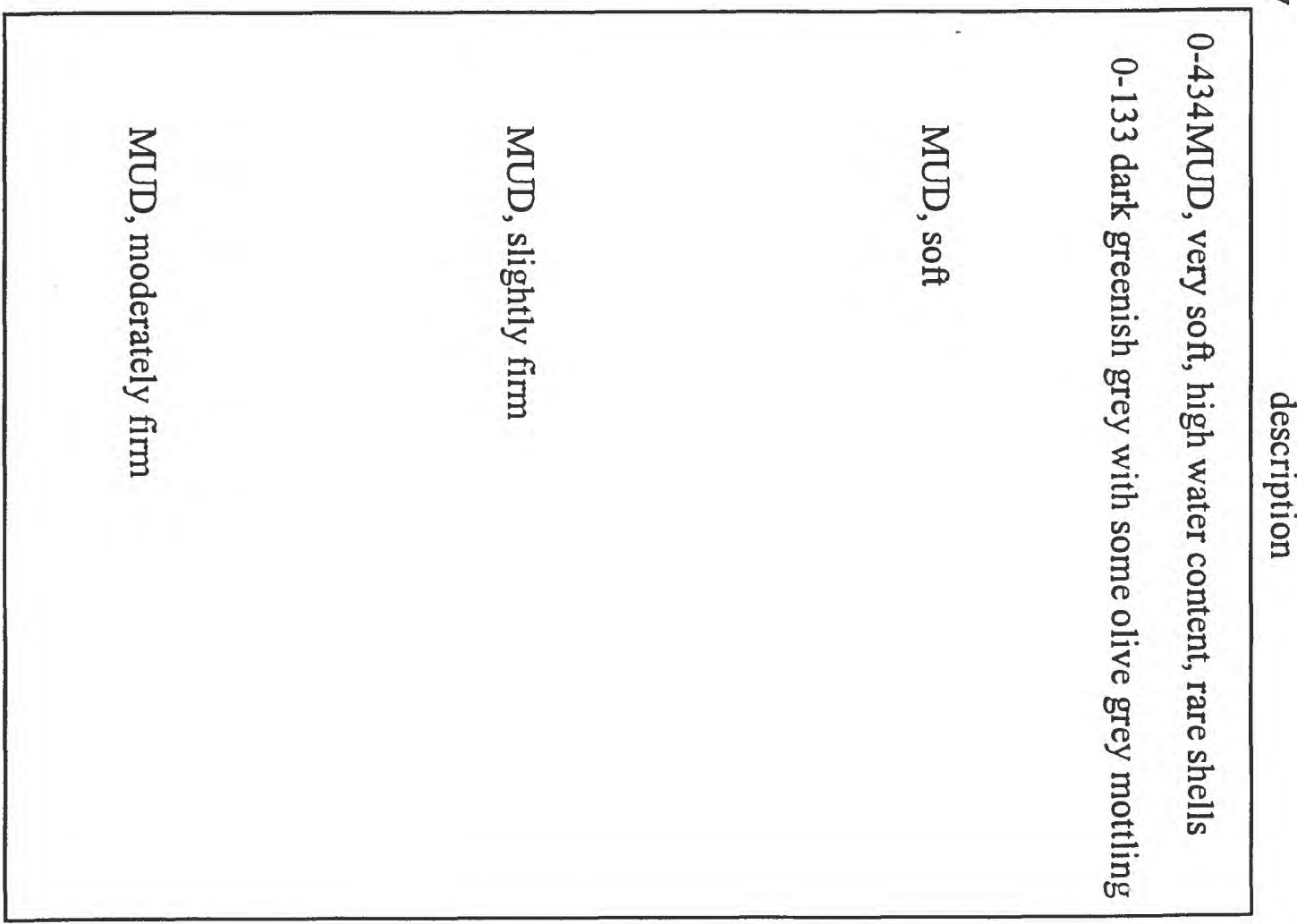



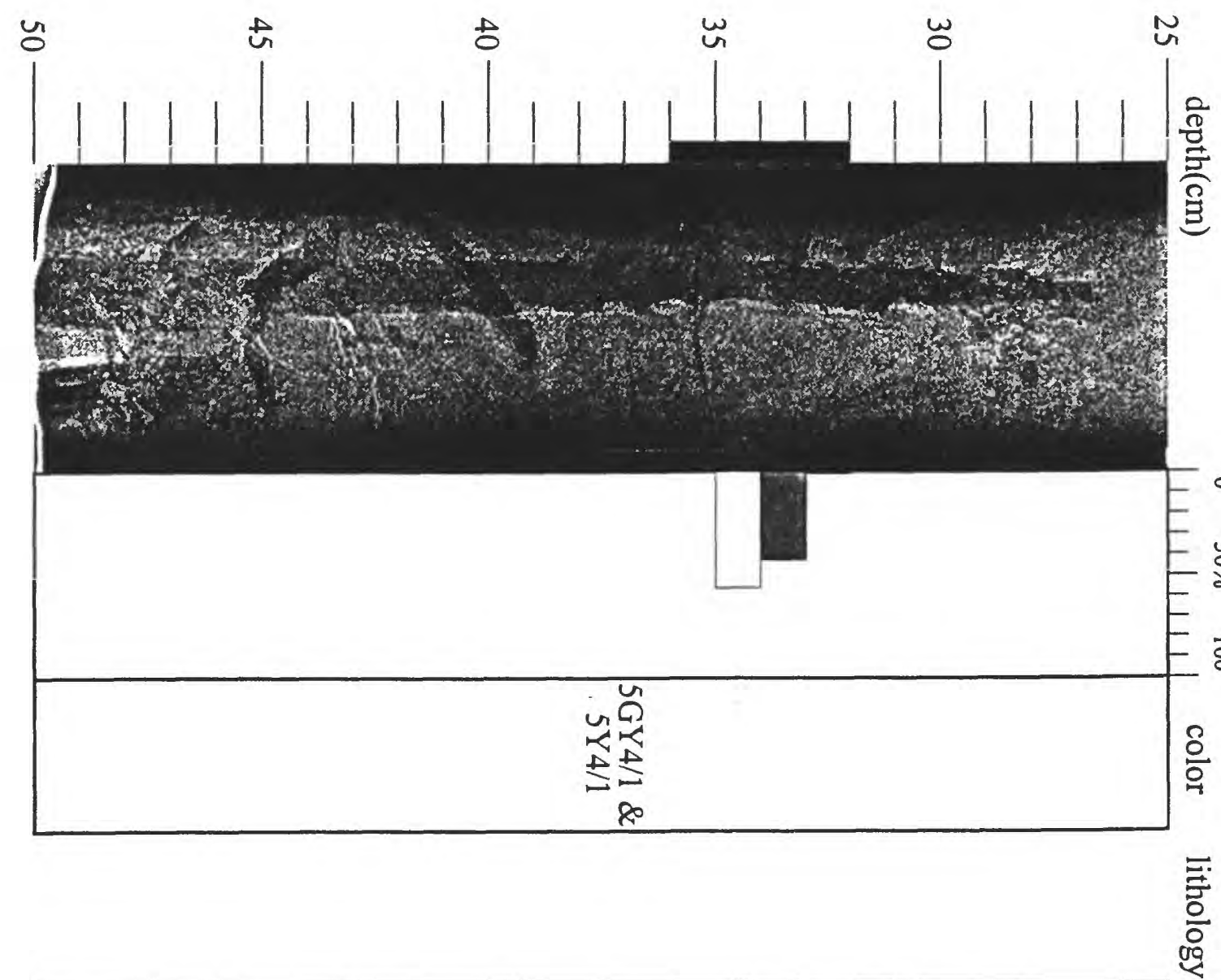

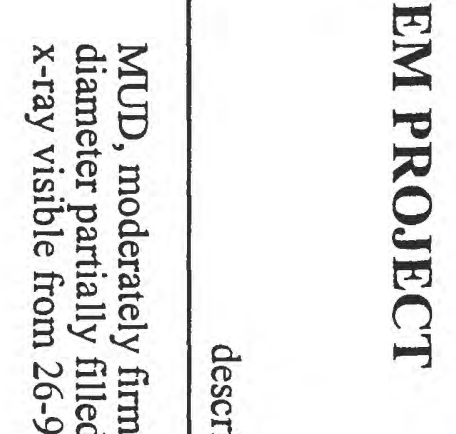

암

声年

\%

\%先

约灵

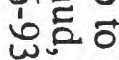

N

$\exists$ 

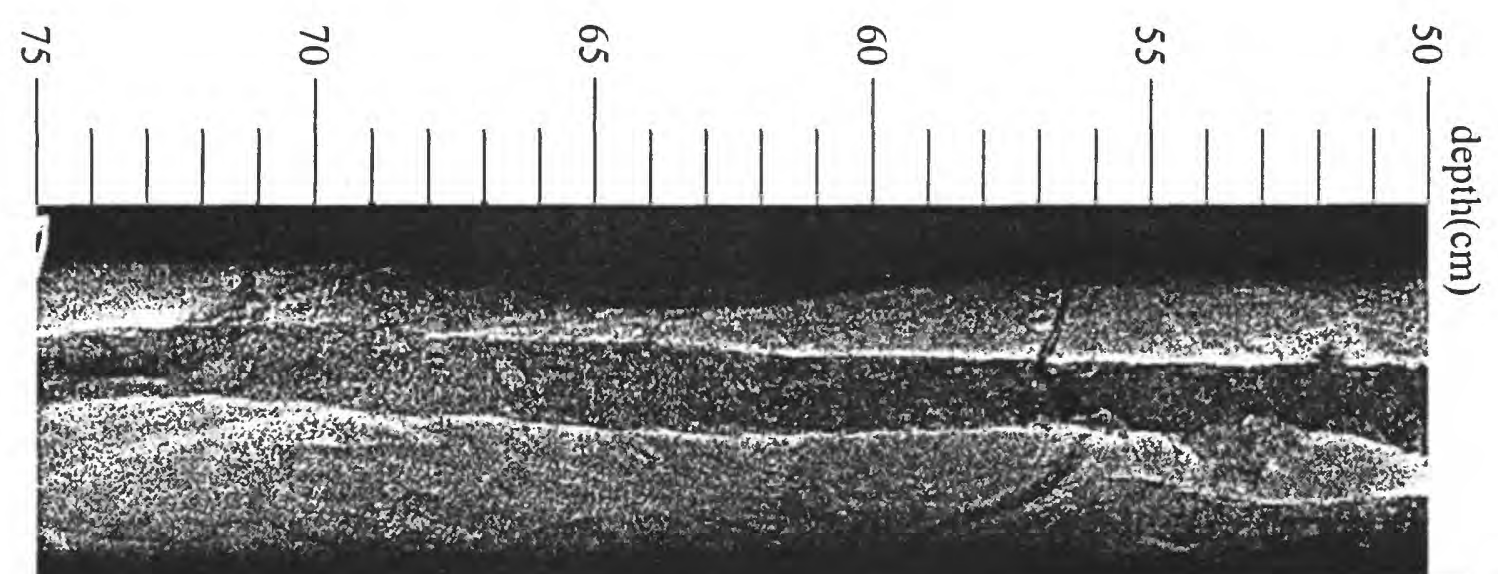

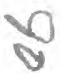

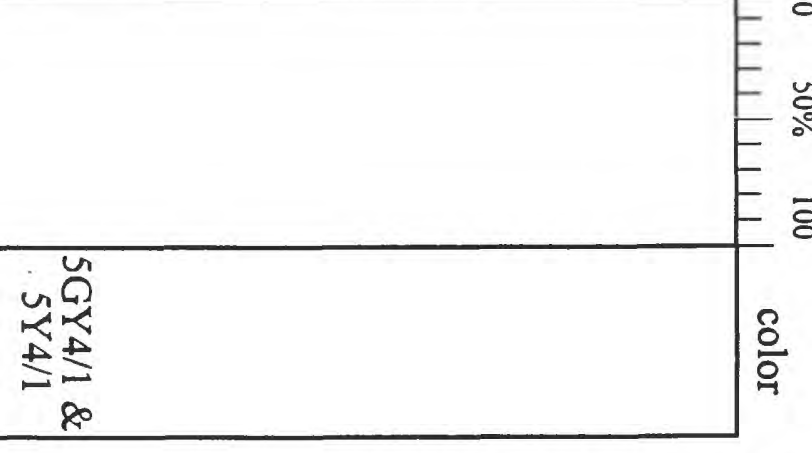

E。

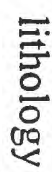

맘

อ

主

官 

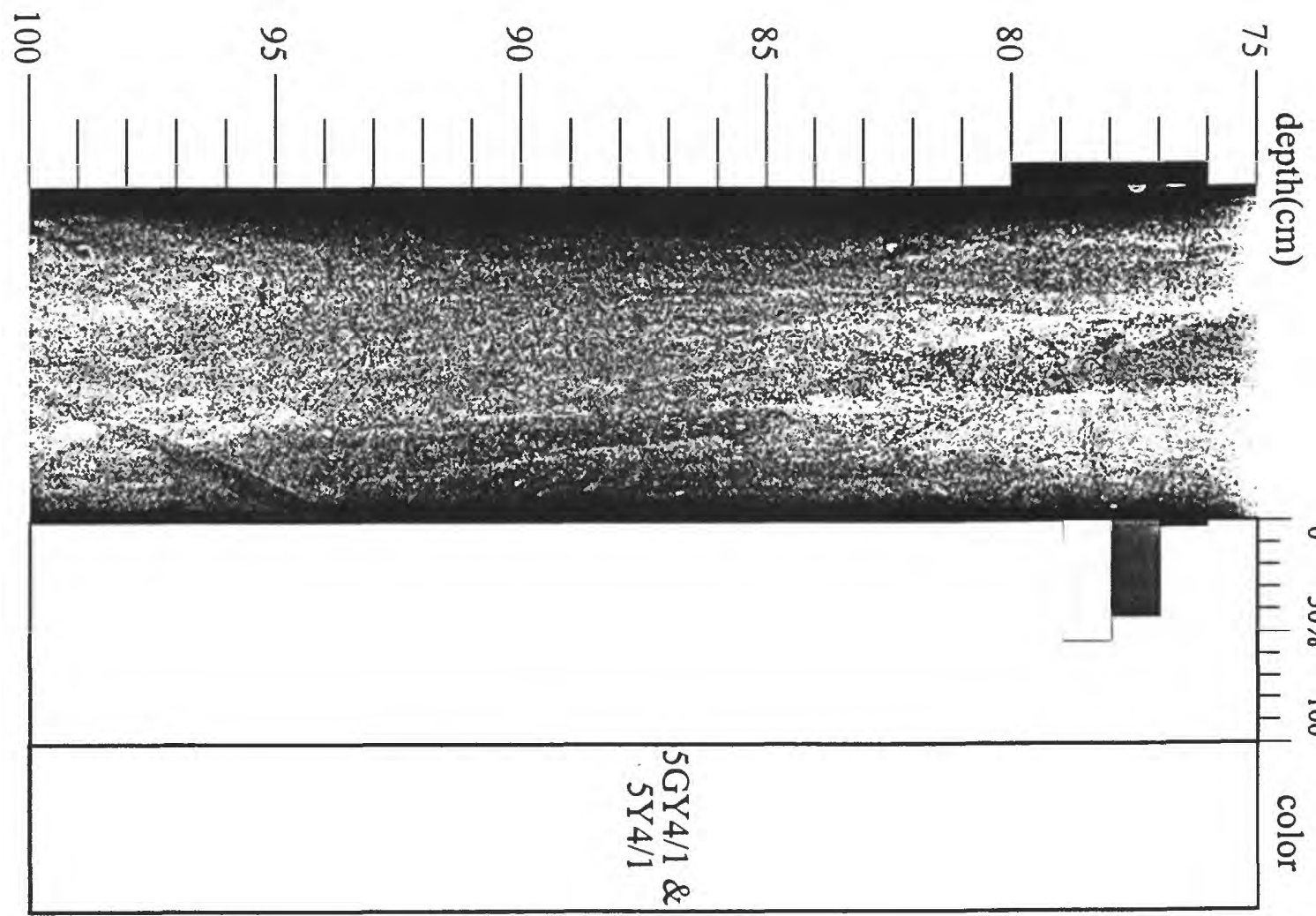

$=\frac{12}{=}=0$

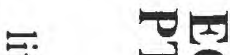

훙
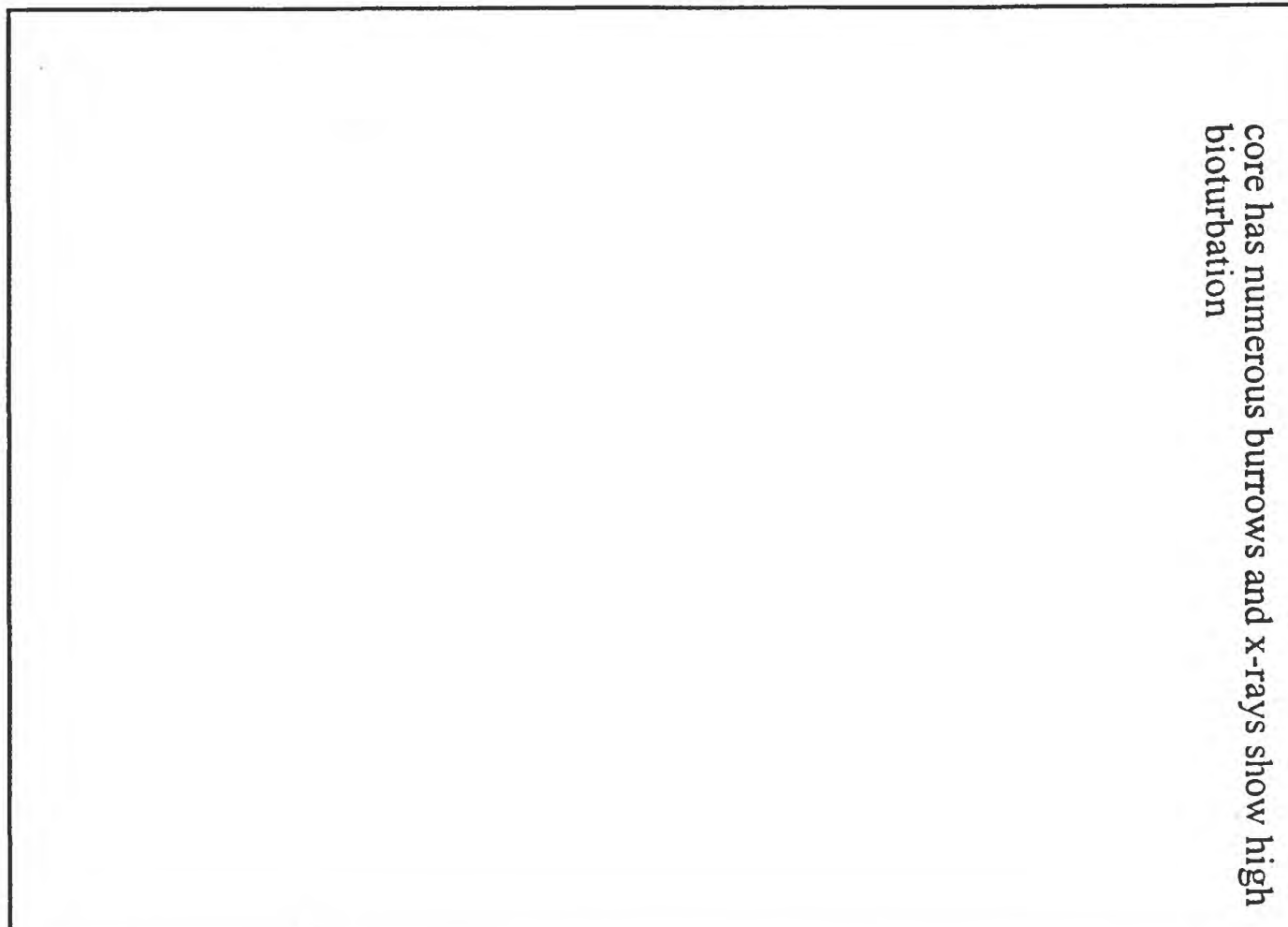

号

怘 


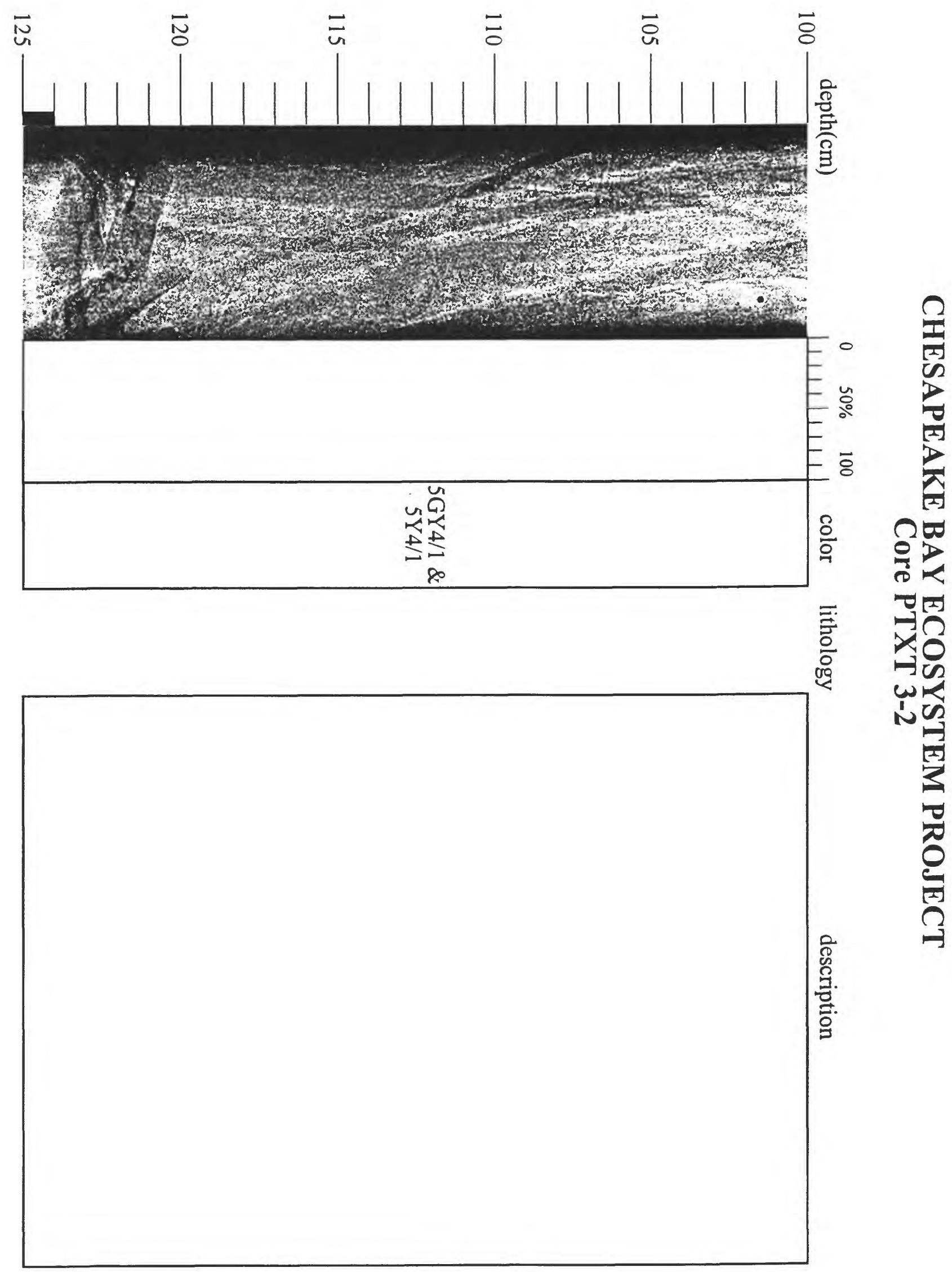



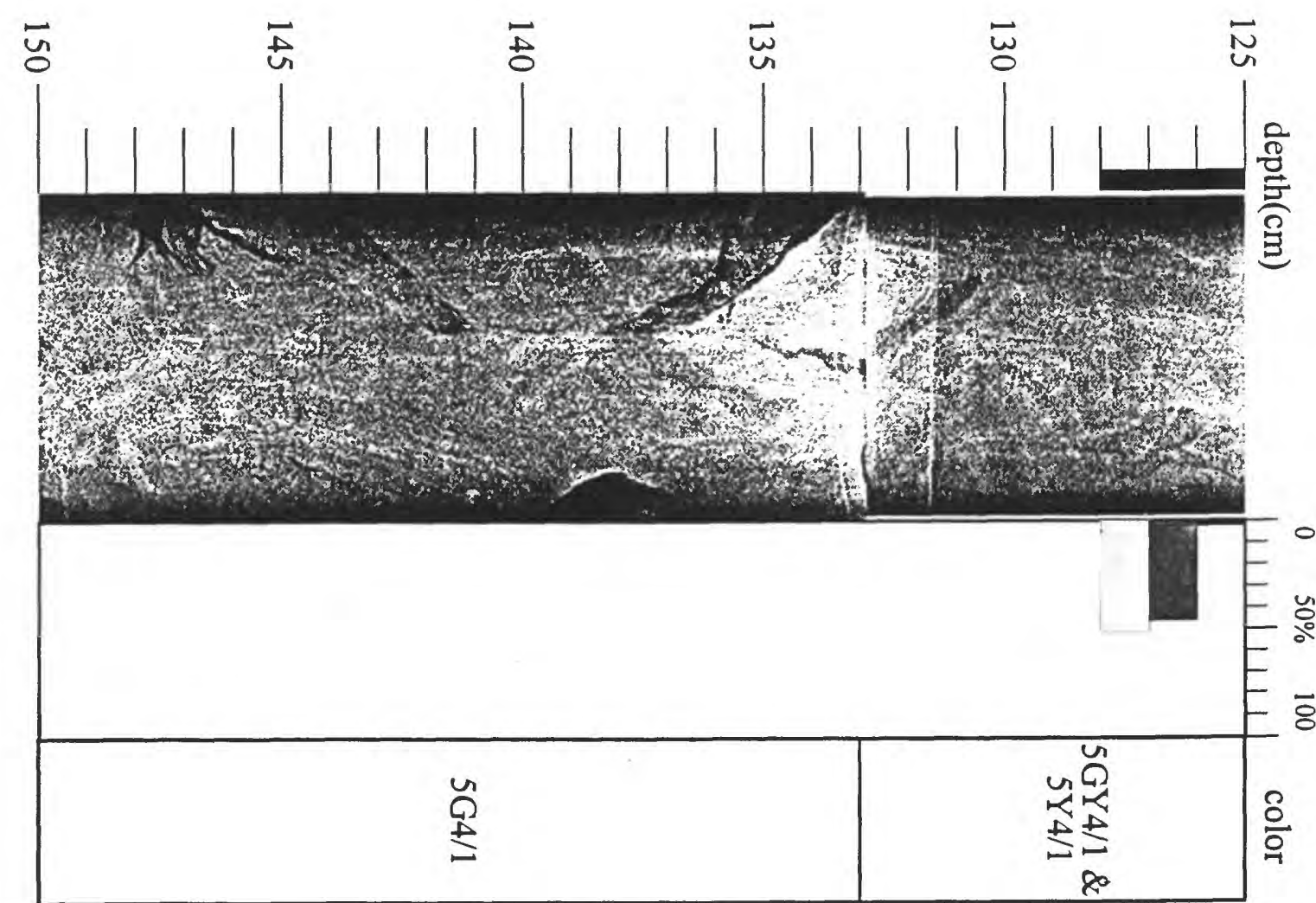

$=0$ -

吾

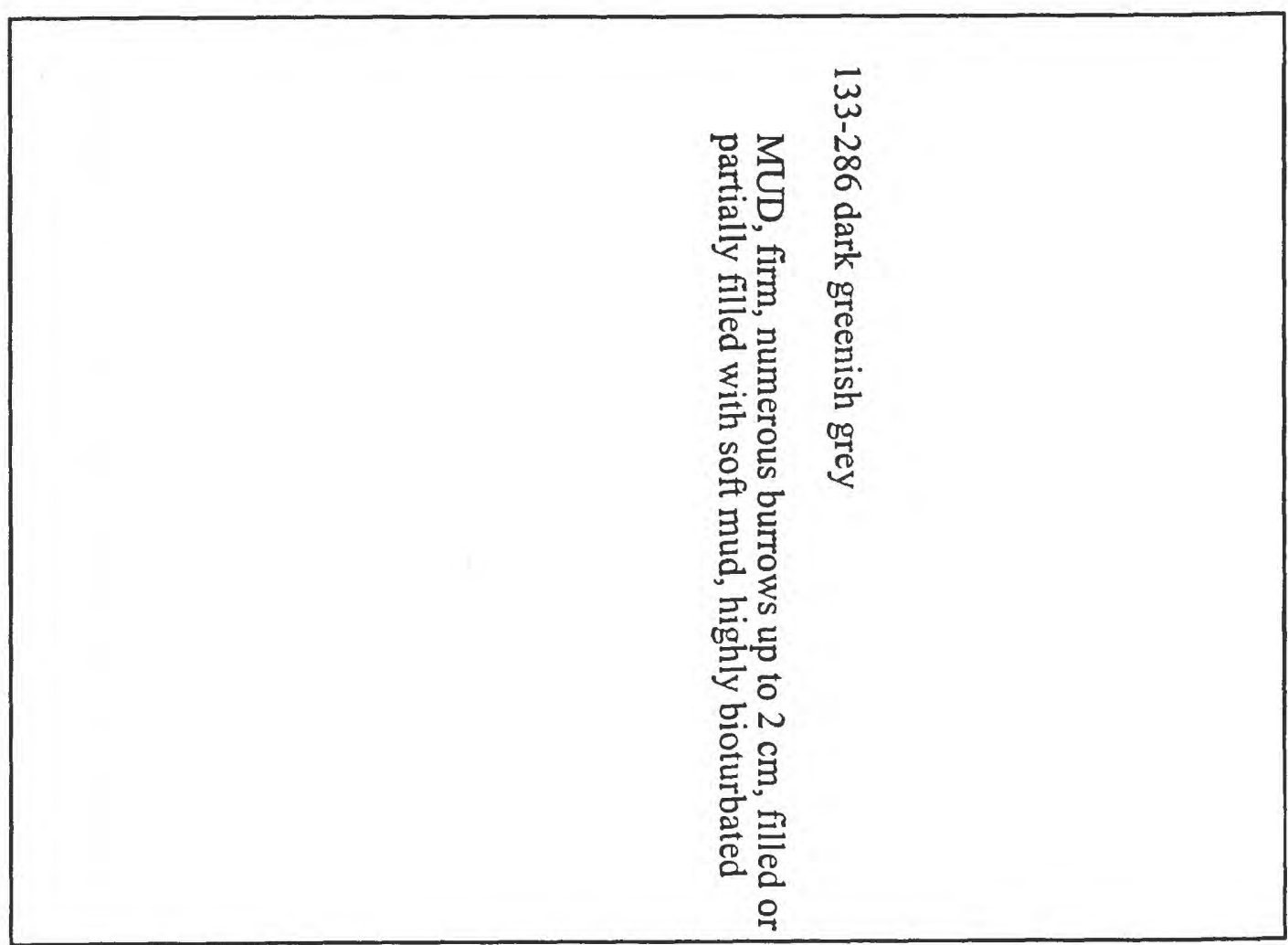



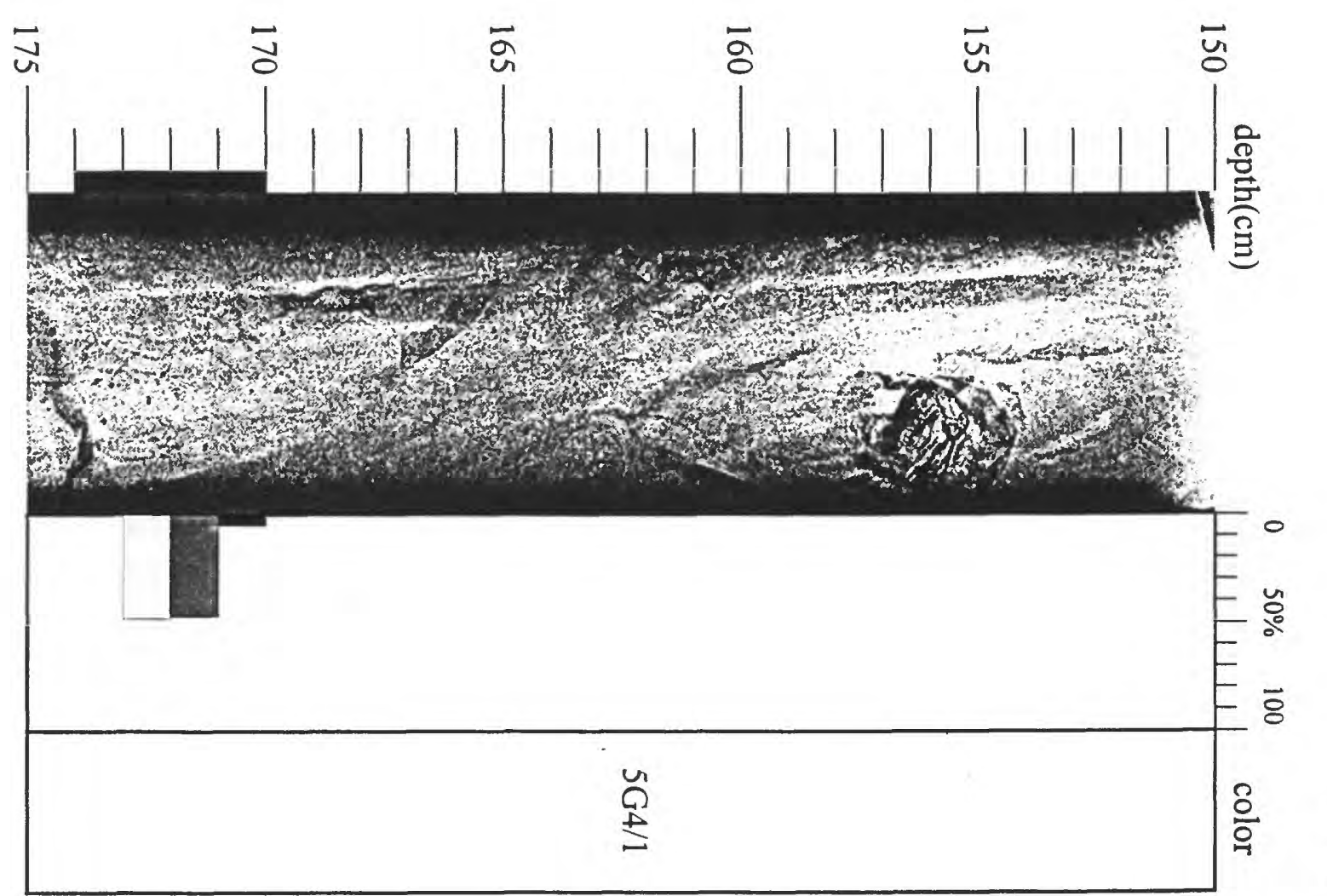

D

콩
음
000

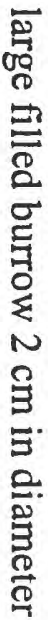

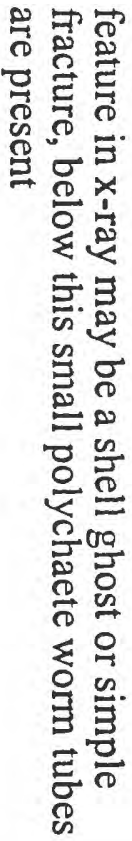



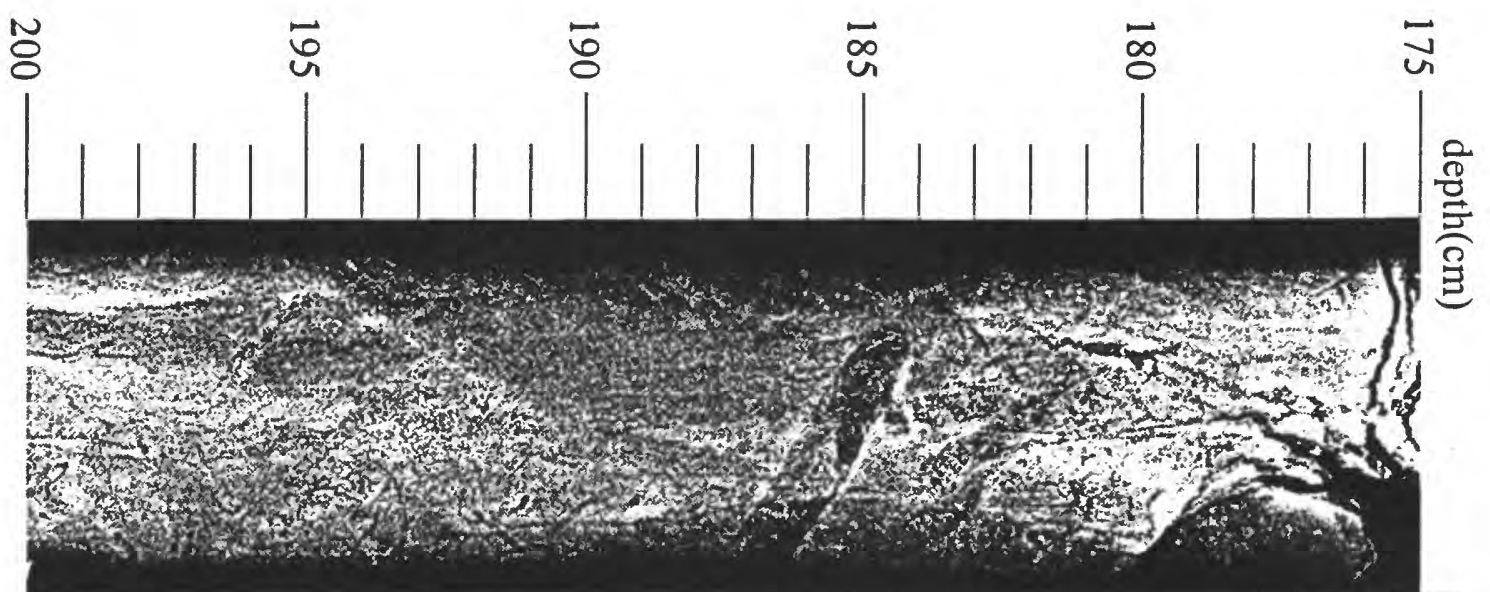

4

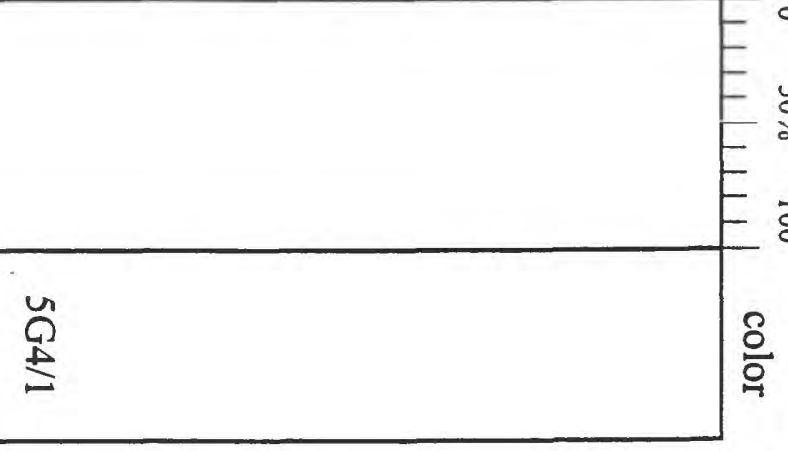

홍

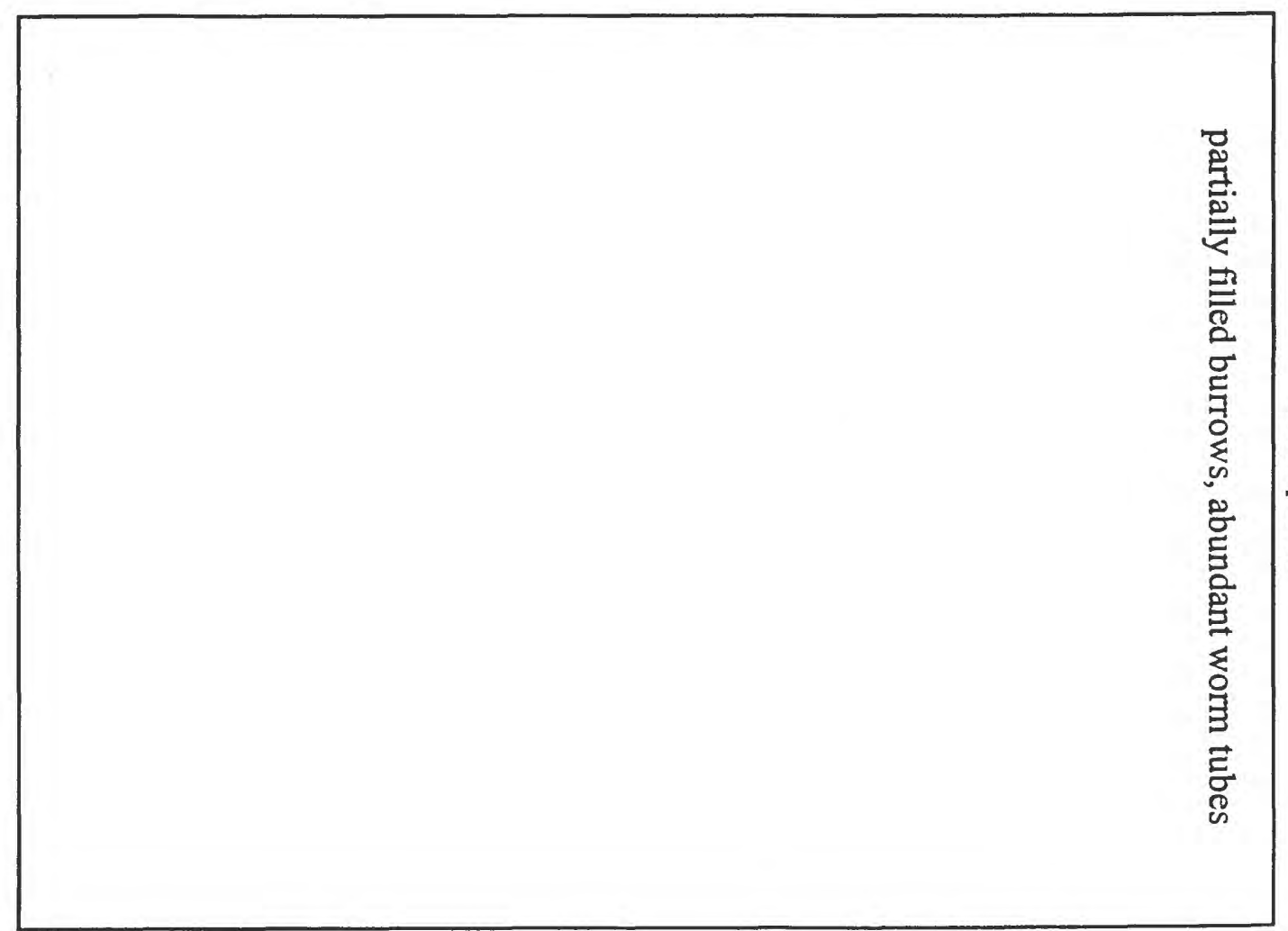




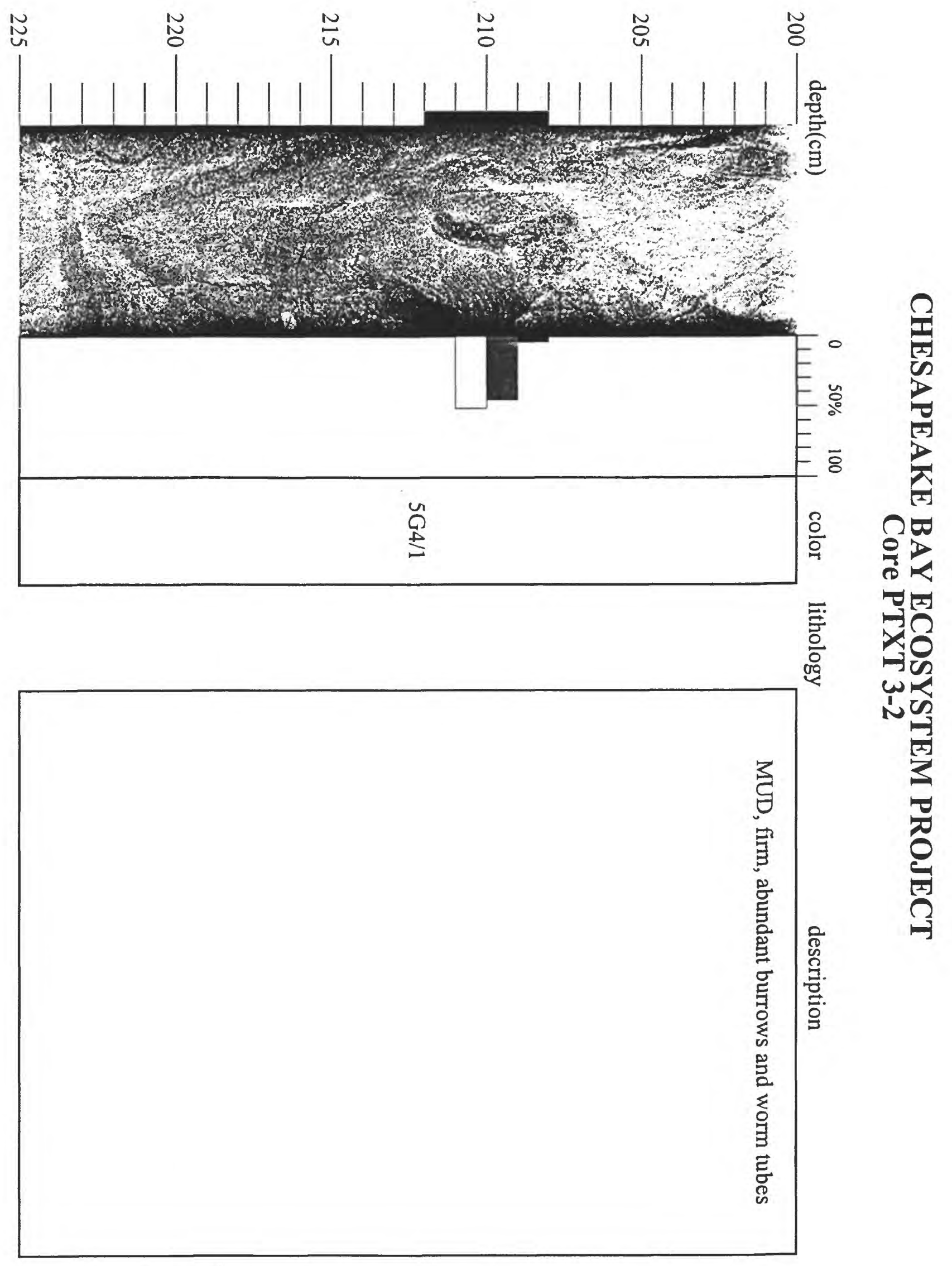




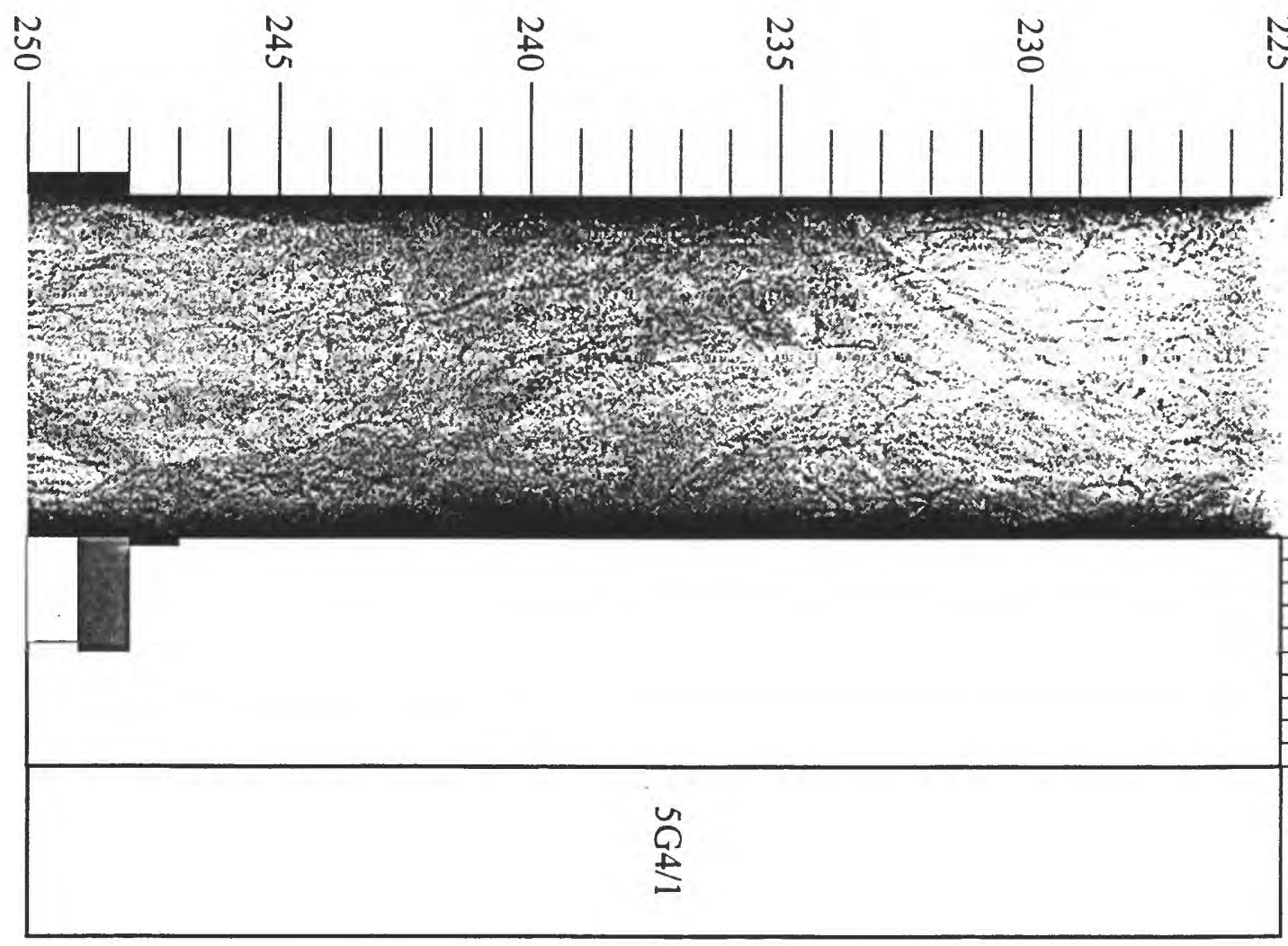

总

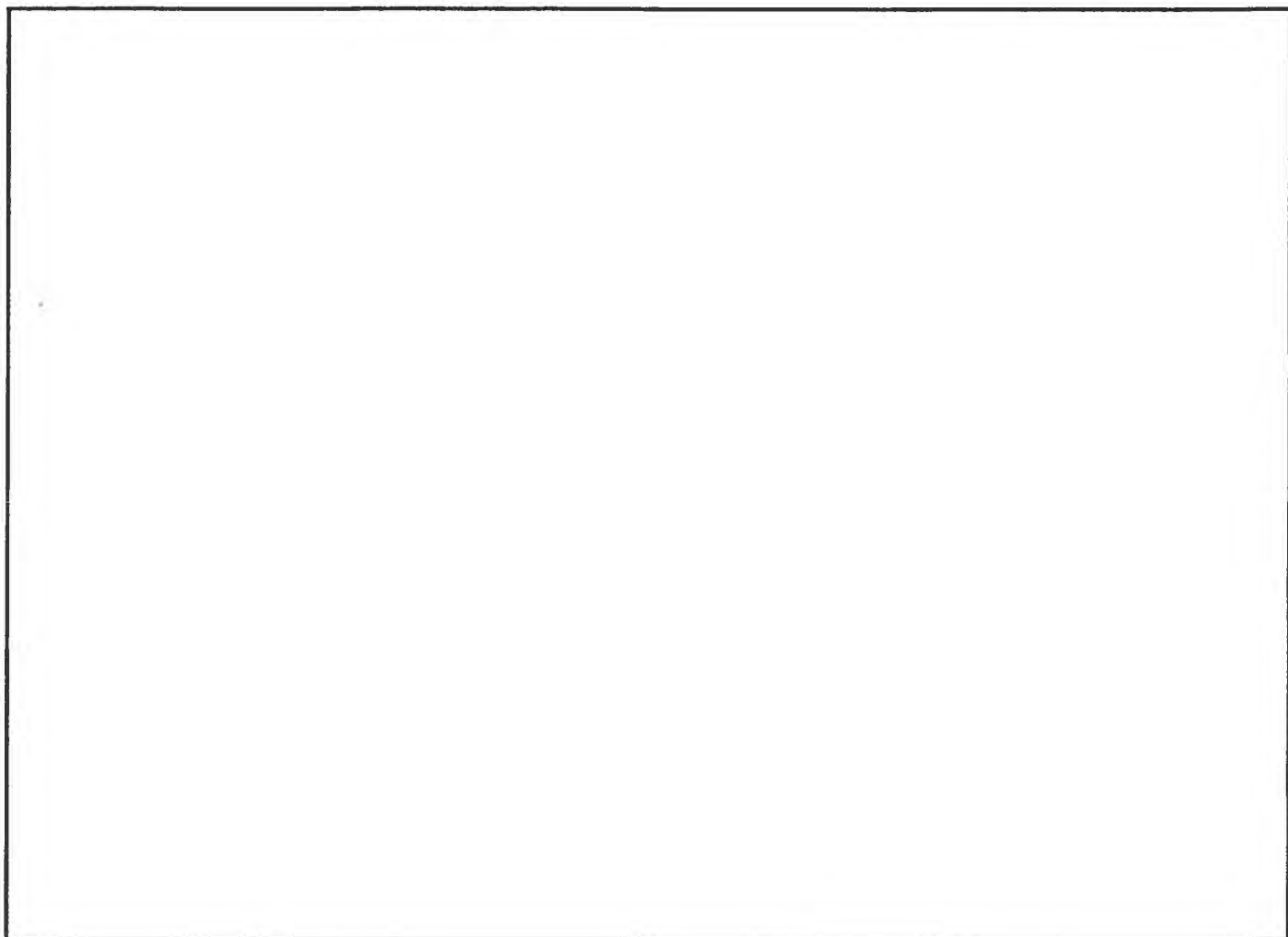

尽. 


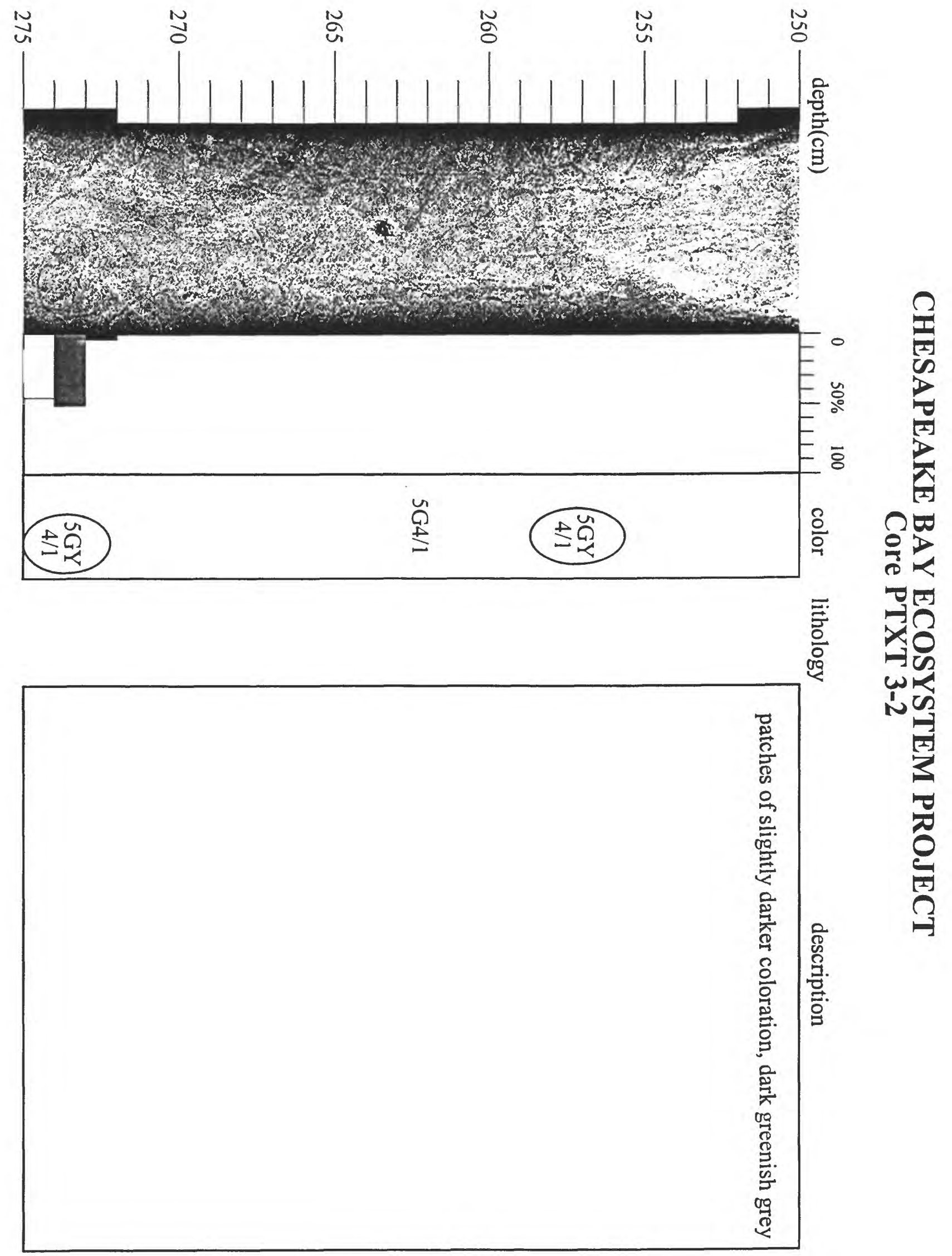




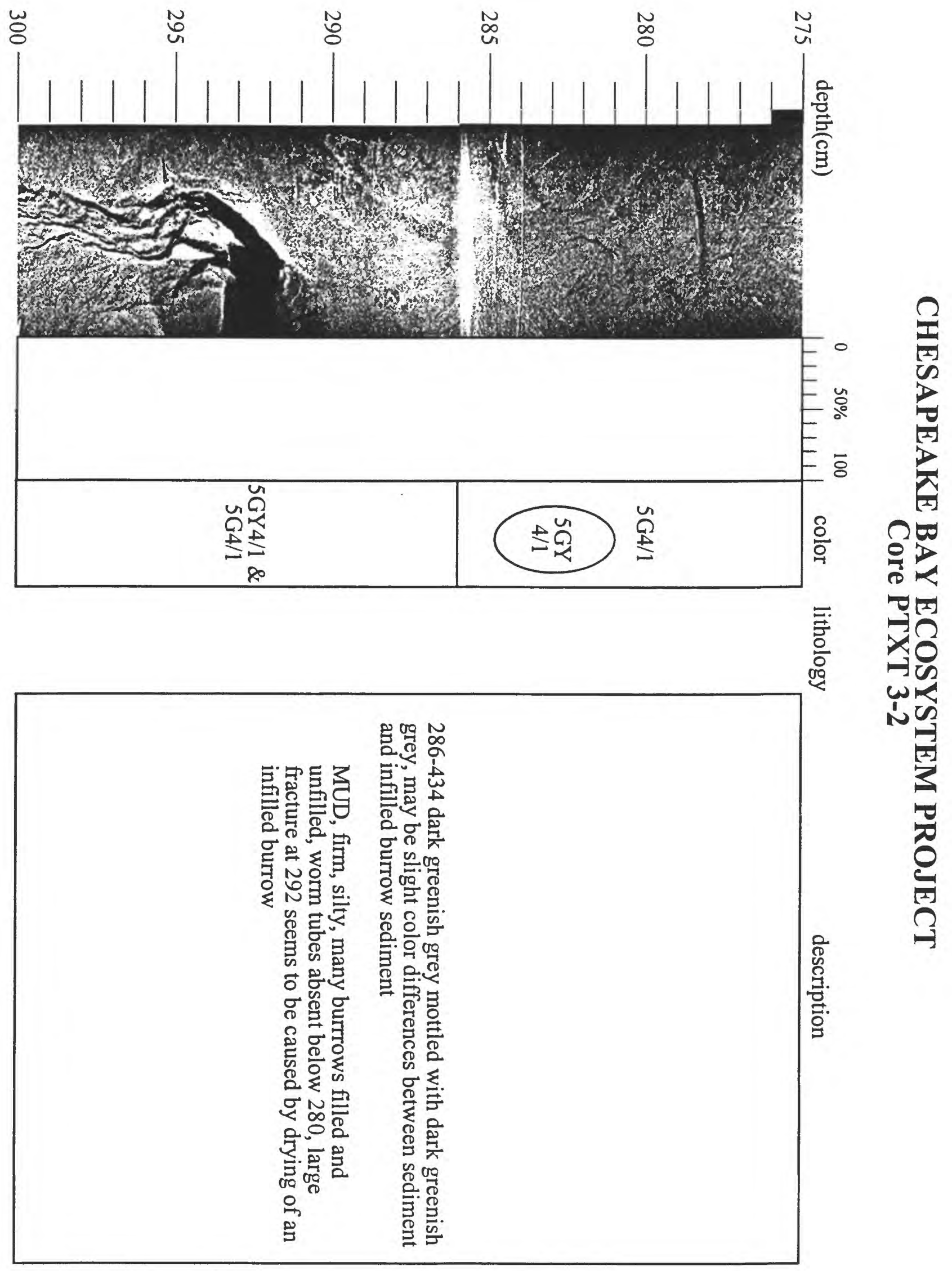



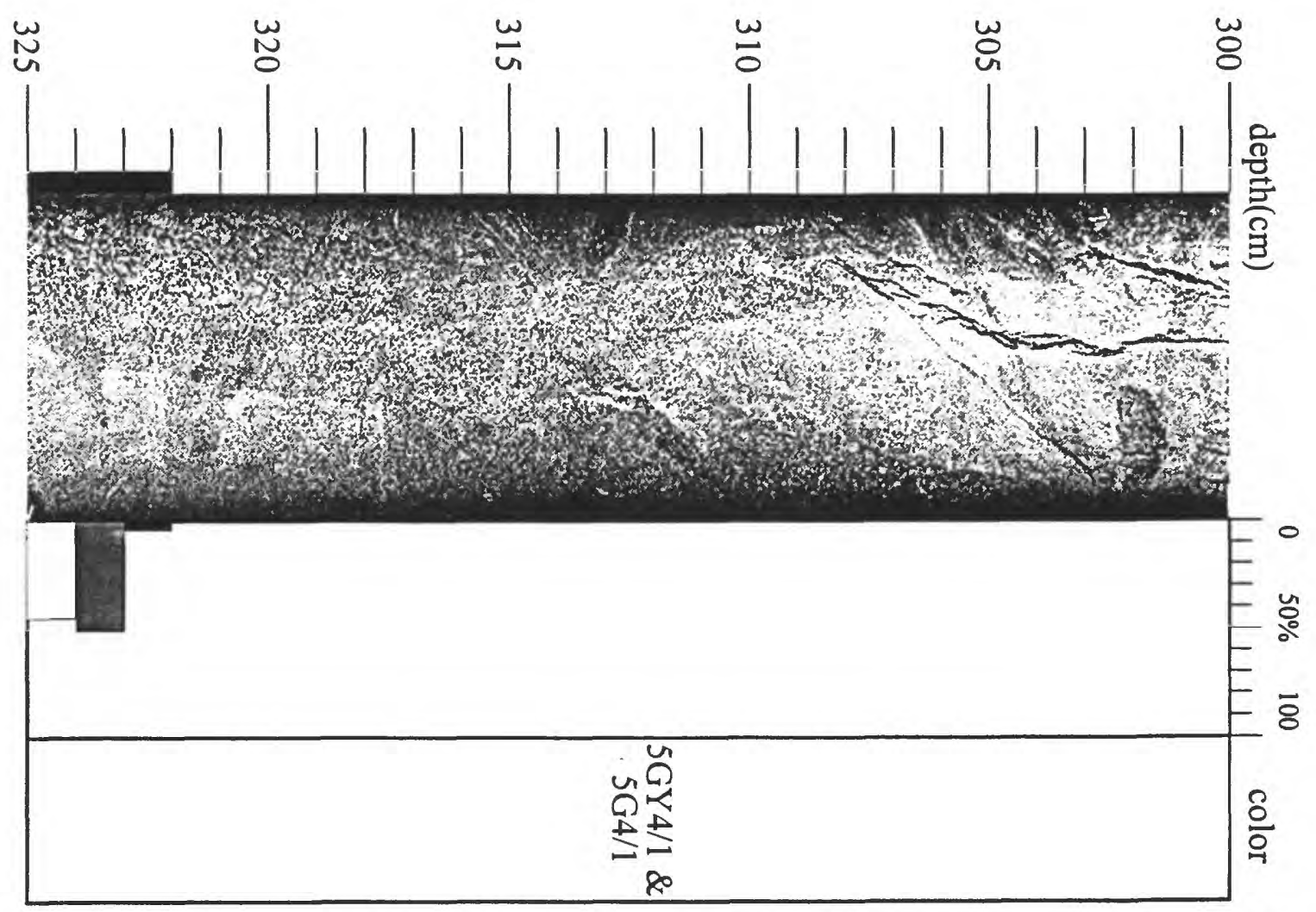

1
0
0

홍

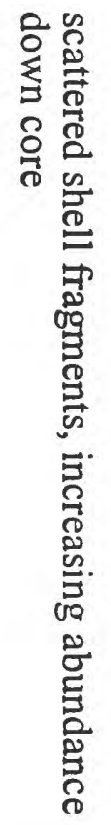

בై

क्र N

อ

요 


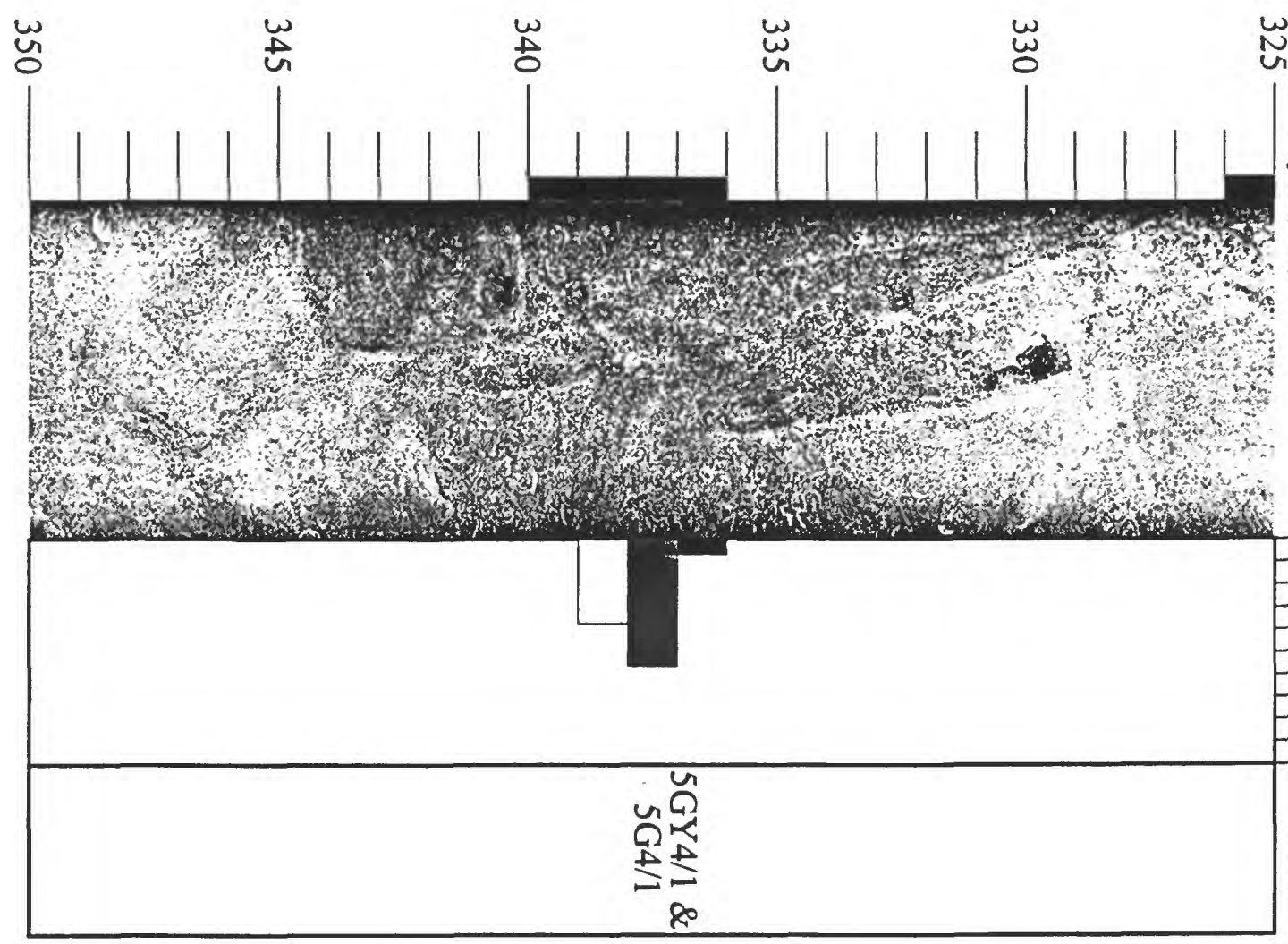

岕

$\frac{0}{3}$

$=\frac{2}{0}=$ :

寄
。
סू

감]

20

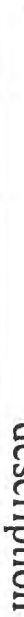

옹. 


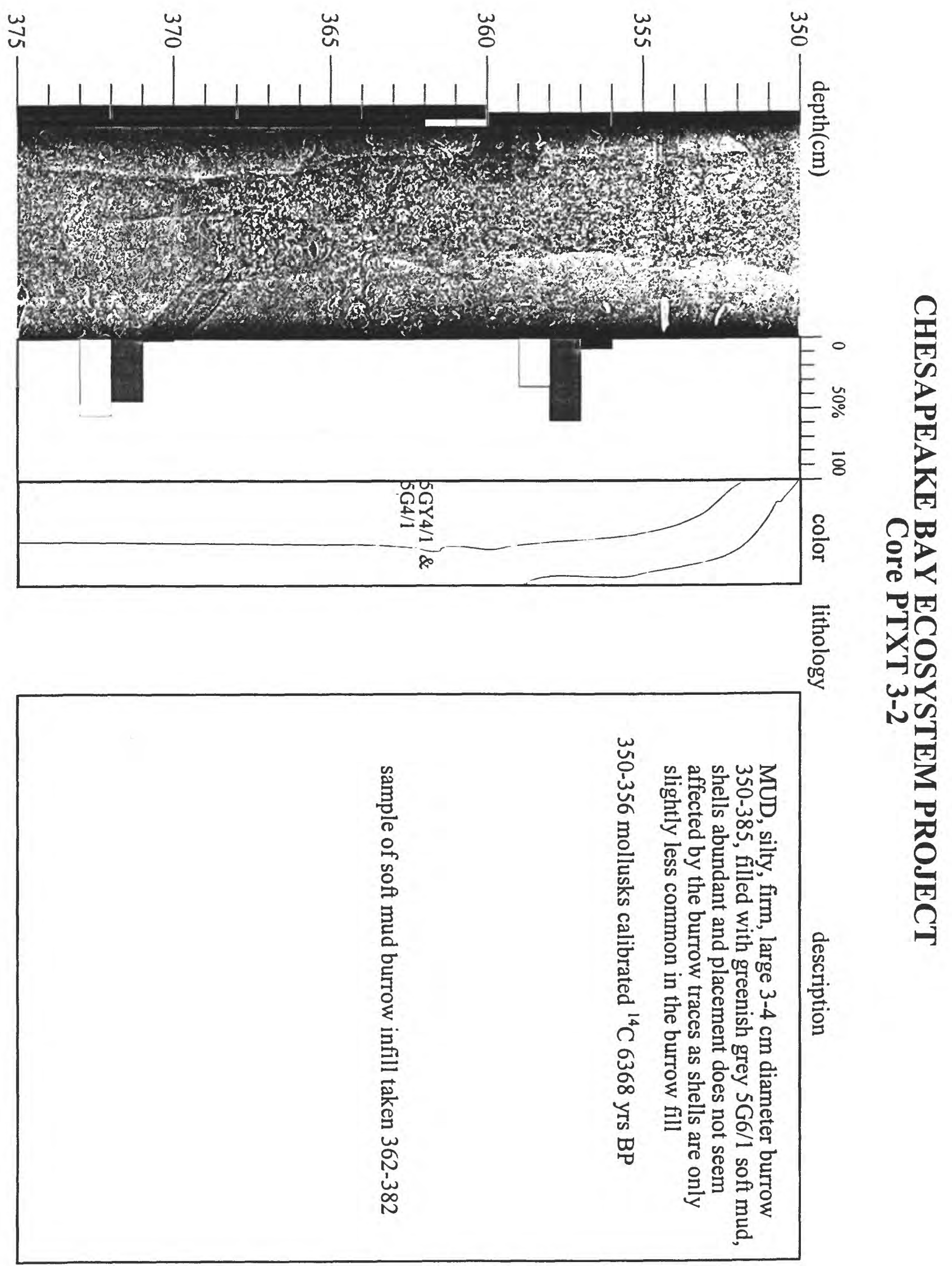




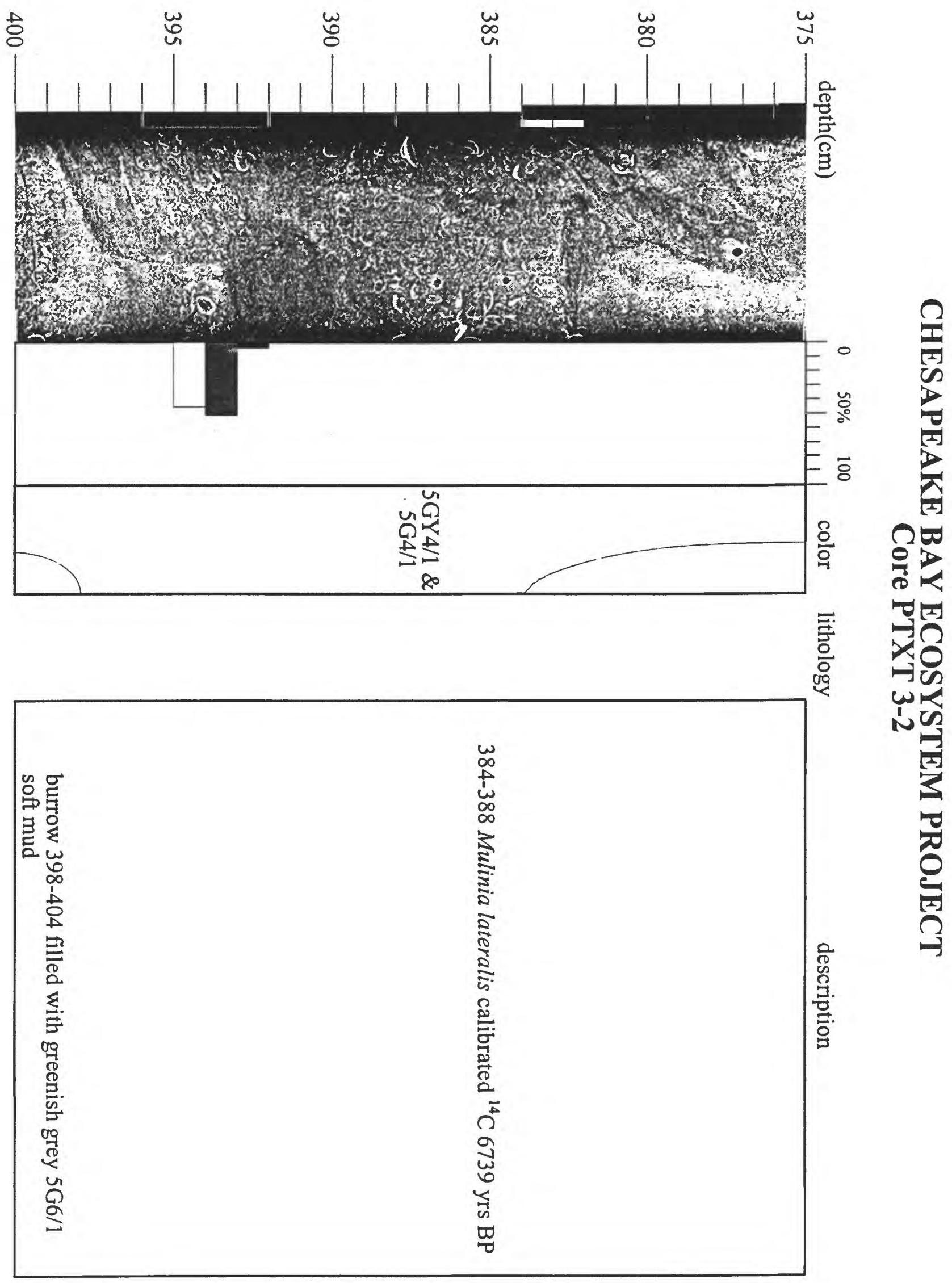



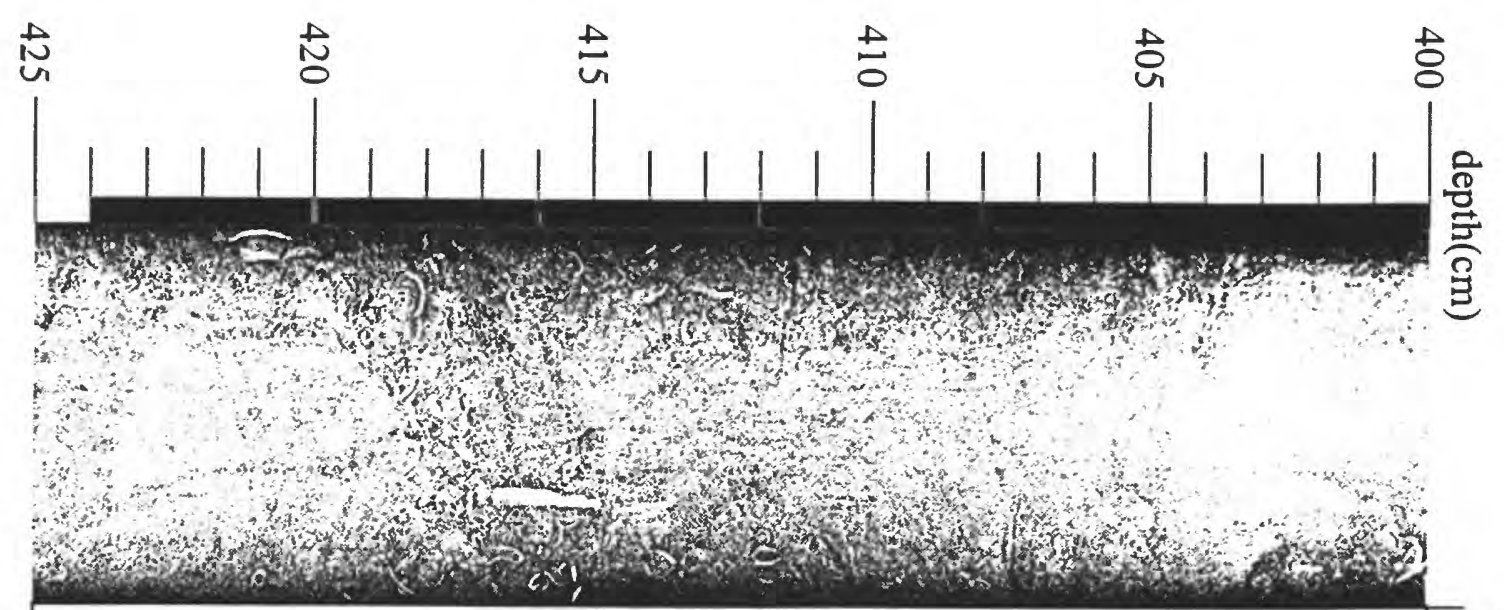

1
$x$
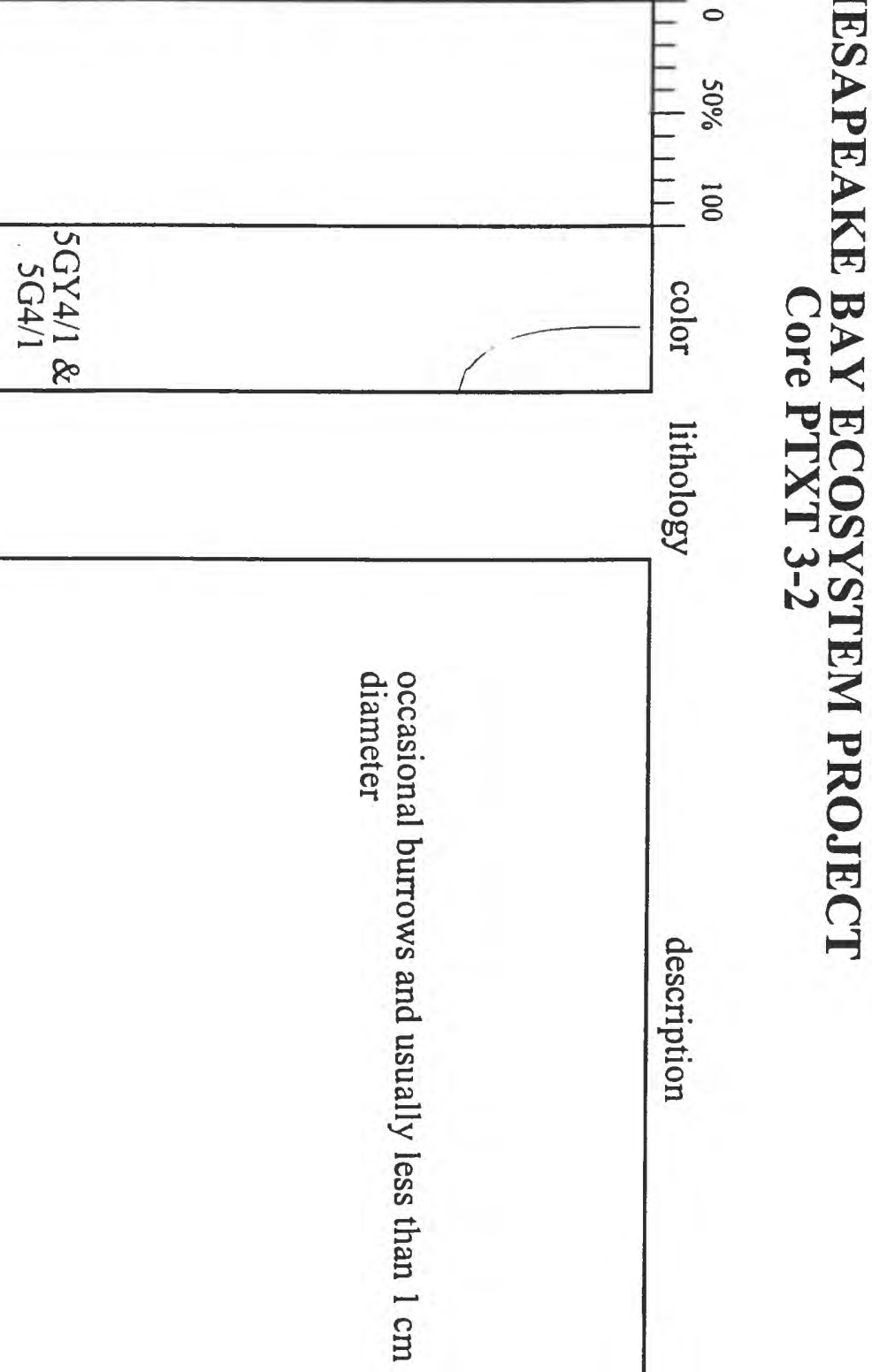

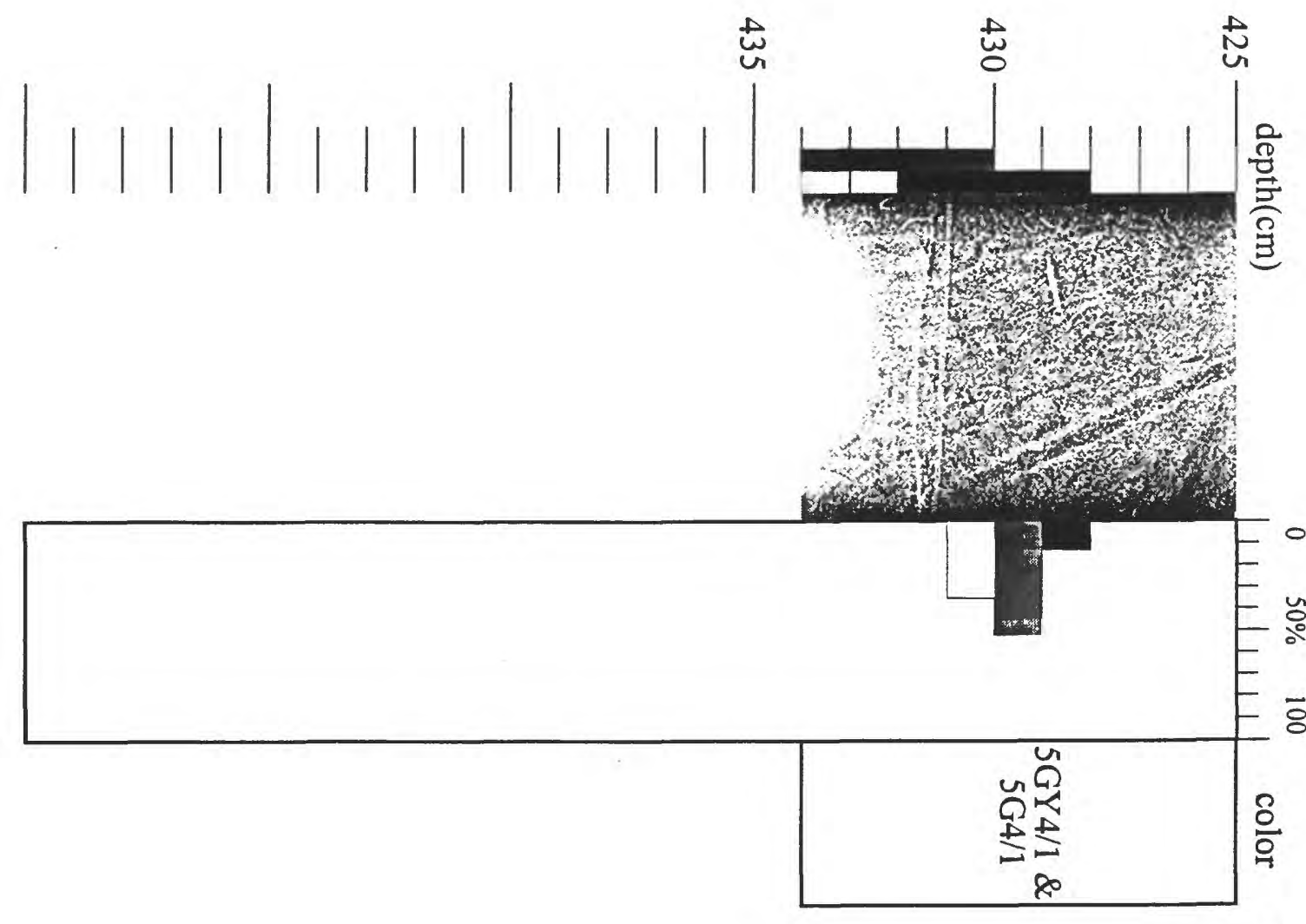

E。

$\mathfrak{Q}_{1}$

盯

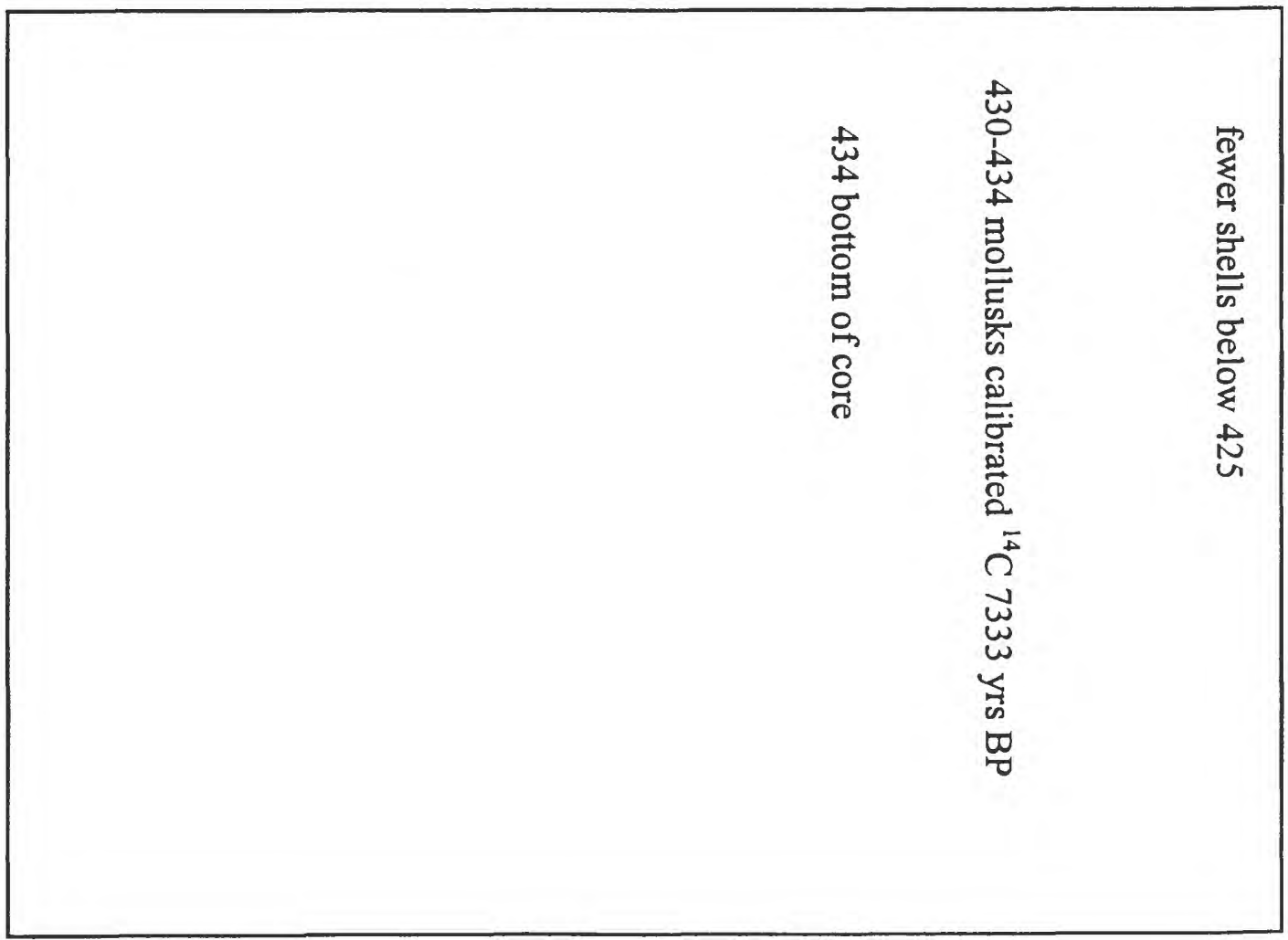




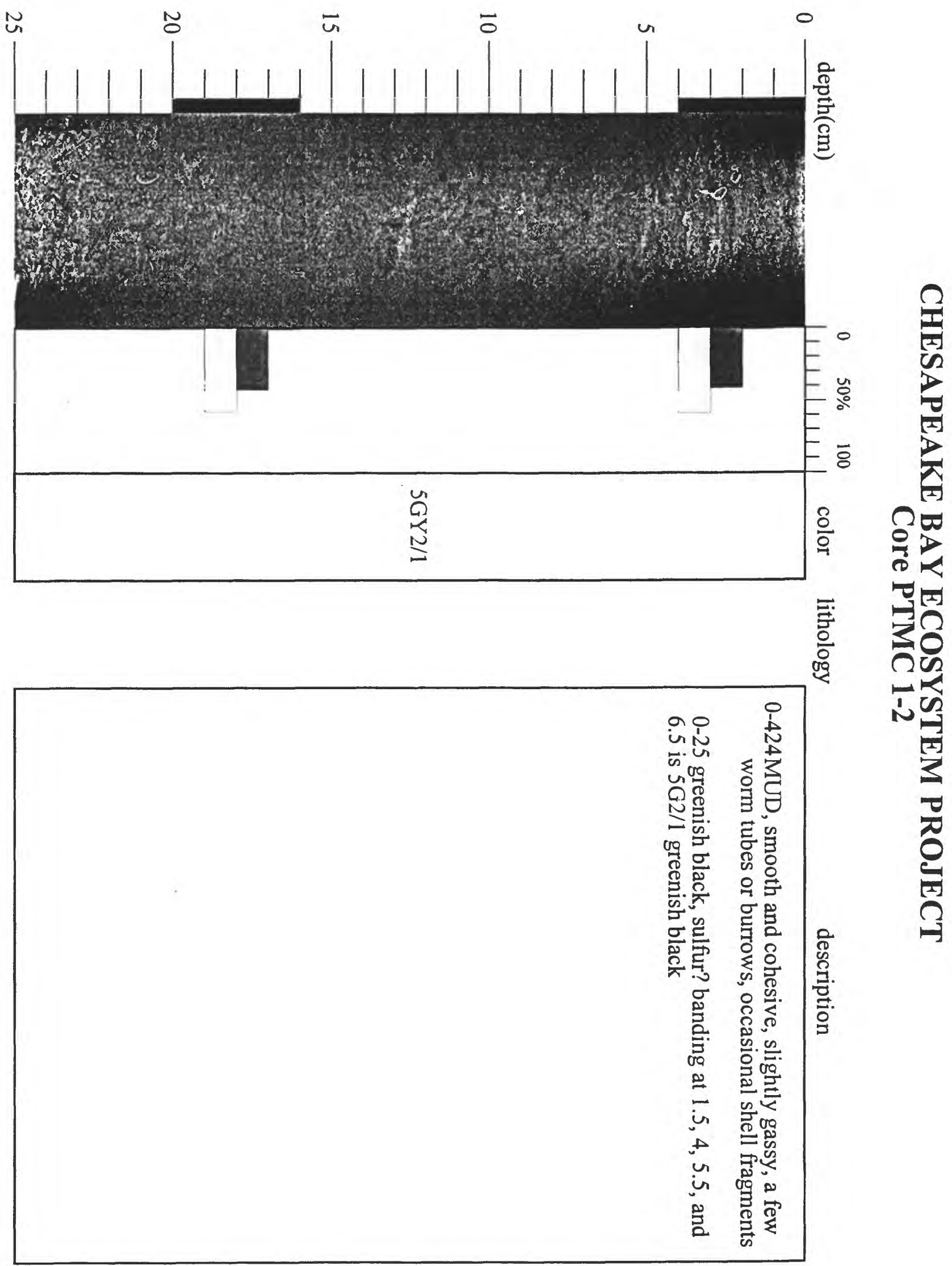



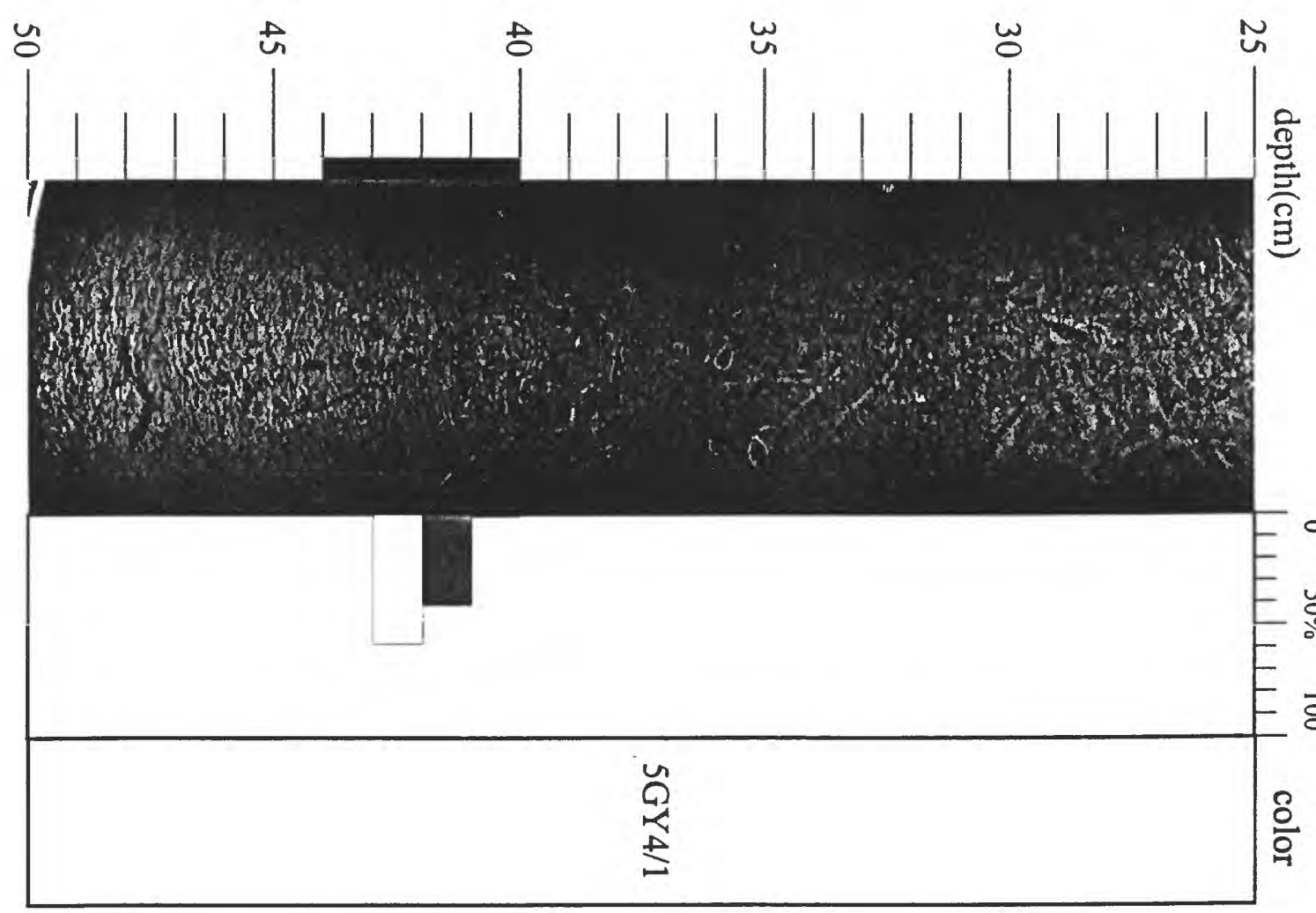

E。

= 아맘

흥

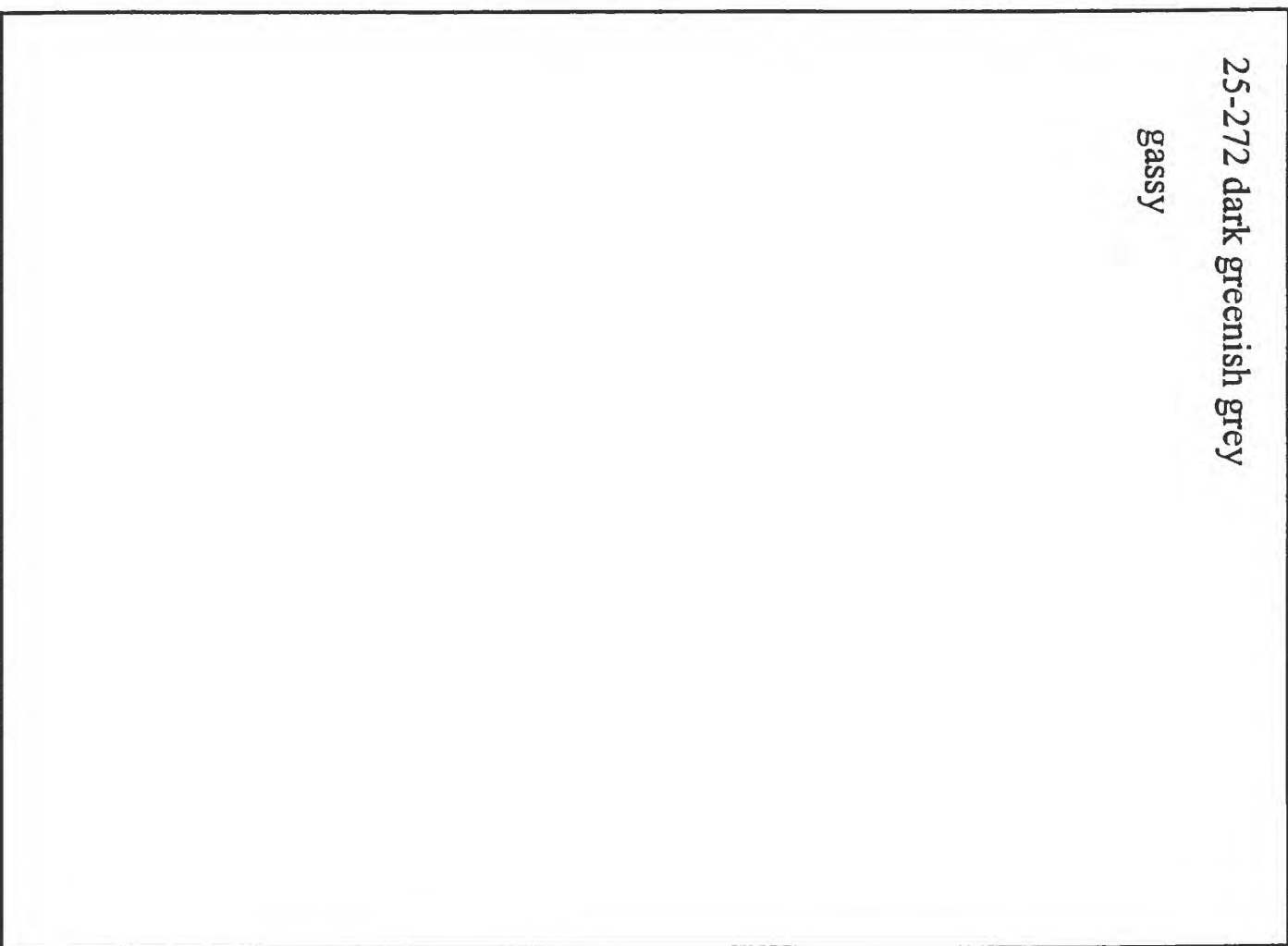




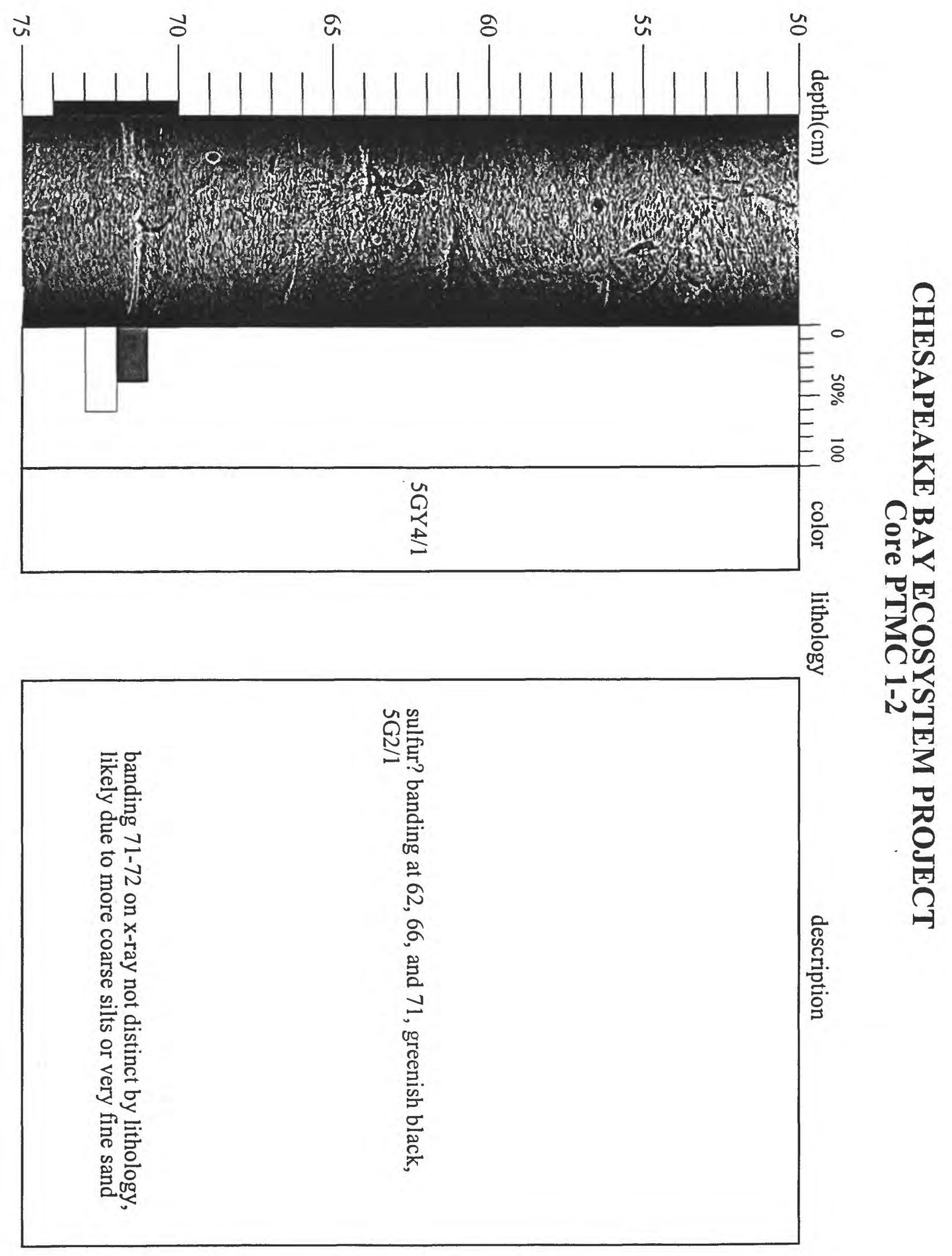




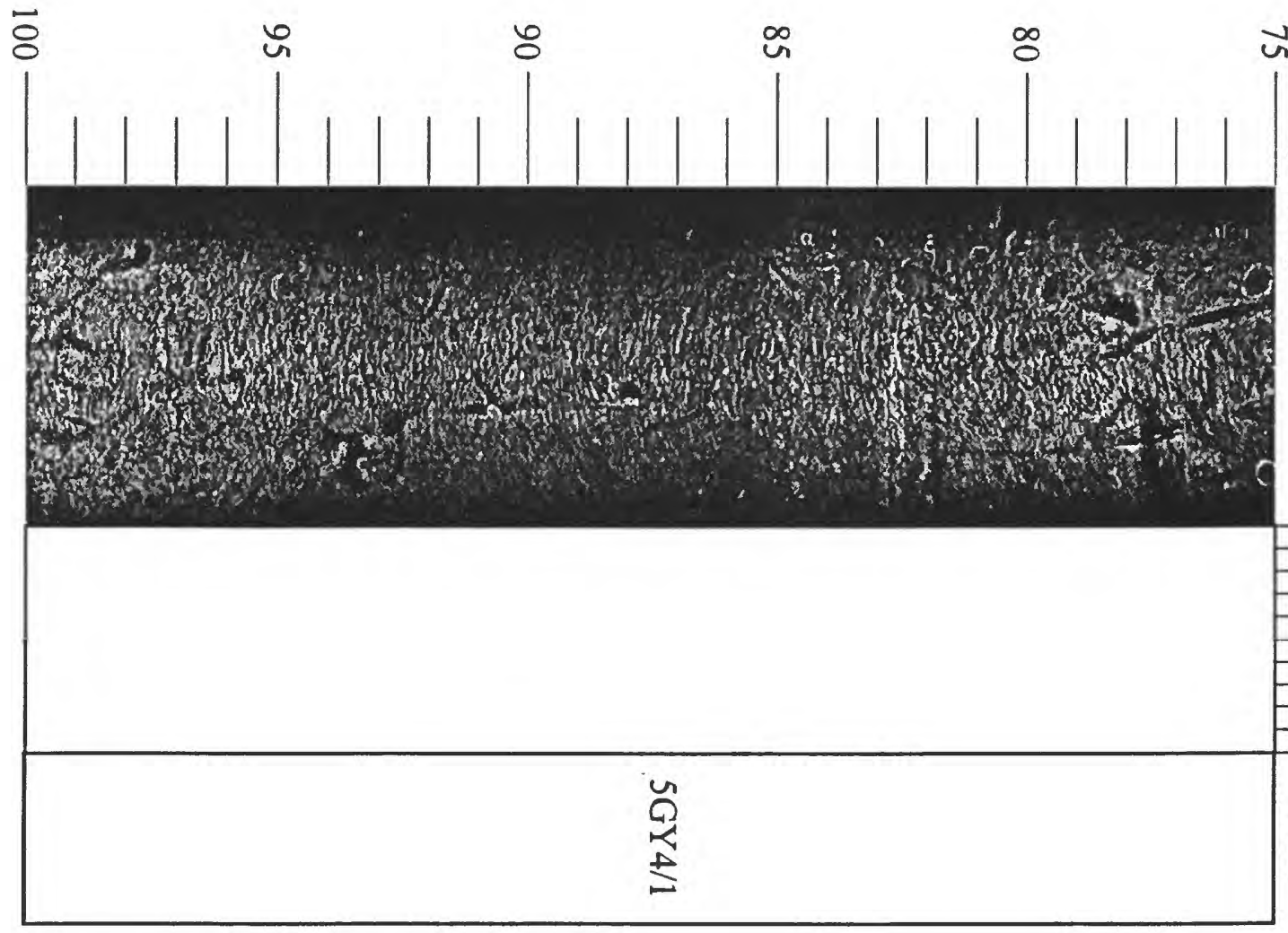

年

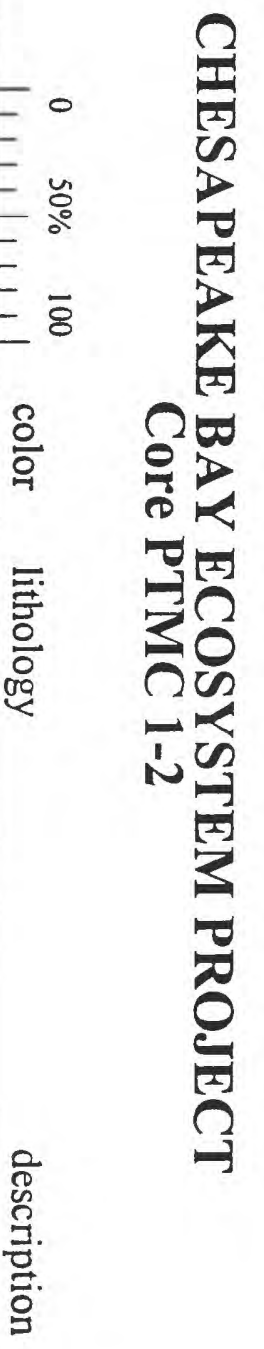




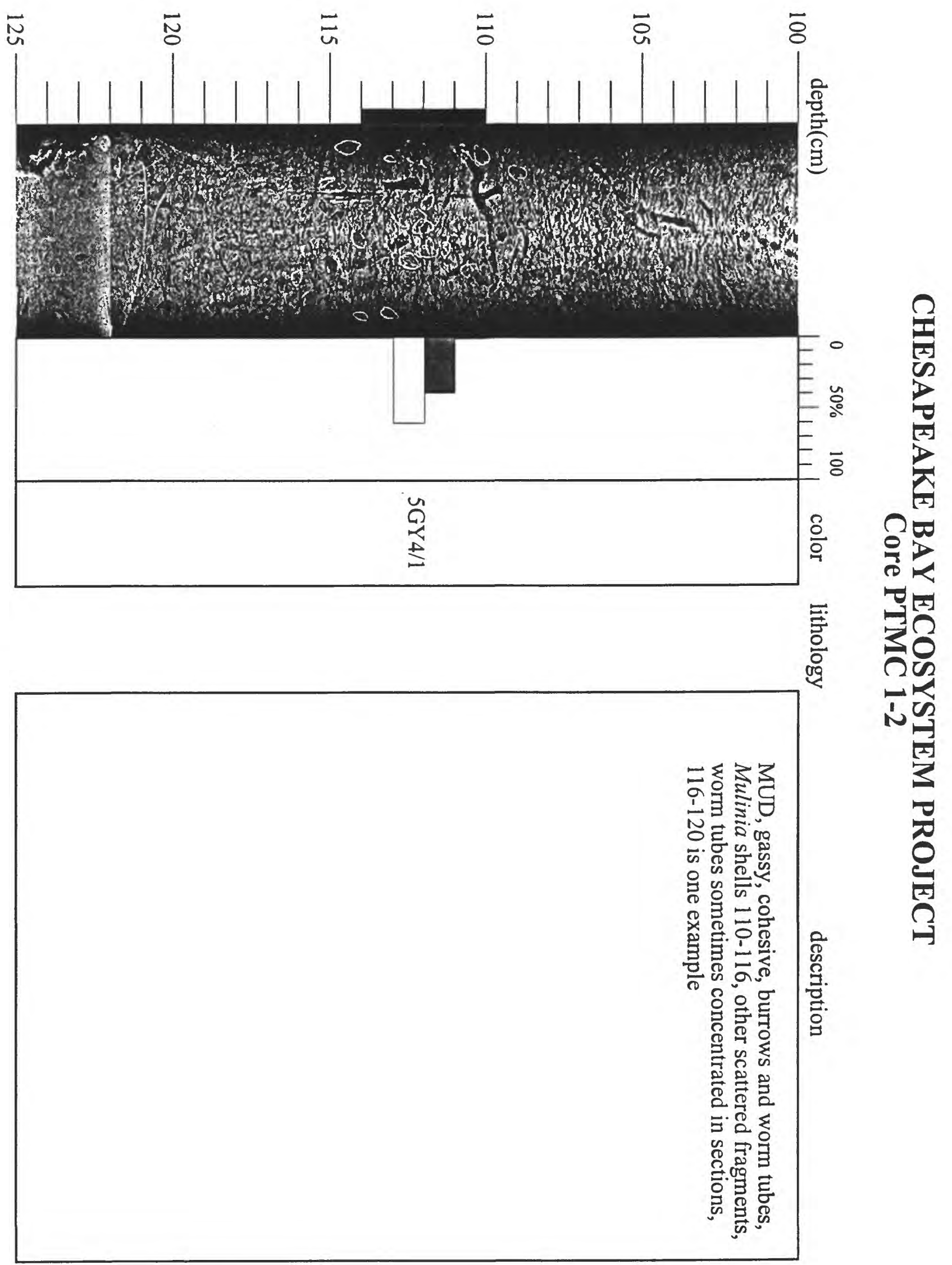




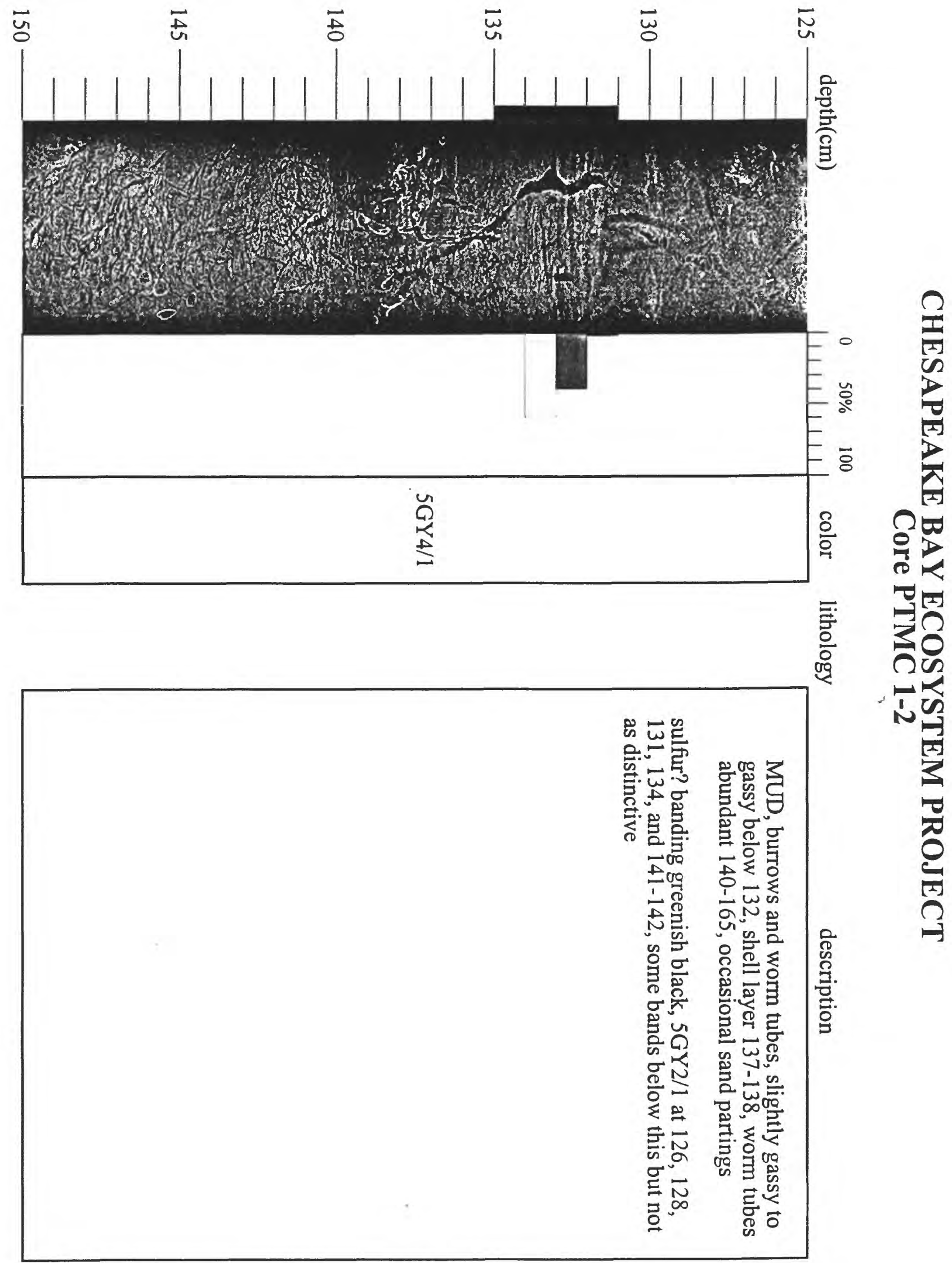




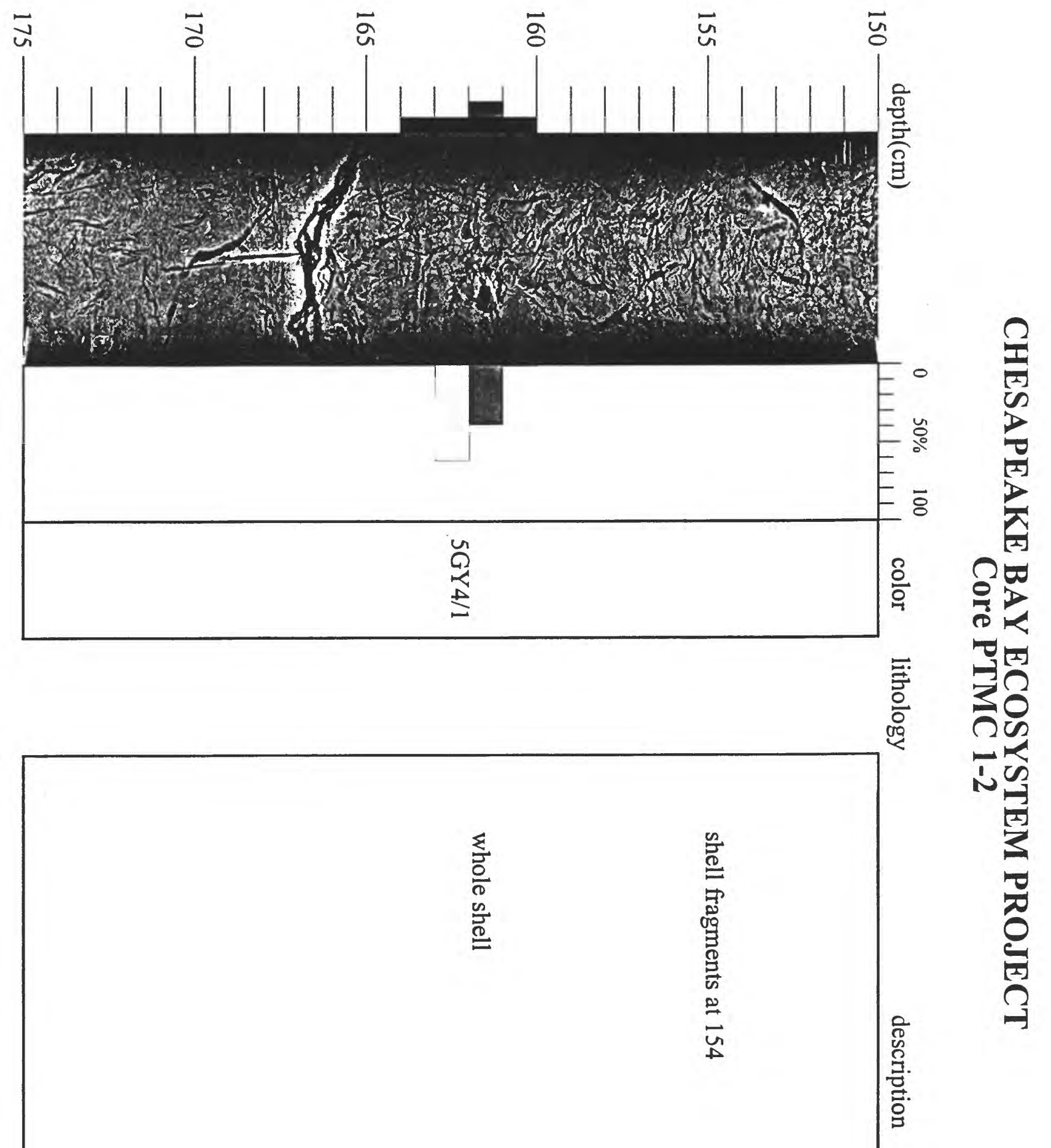



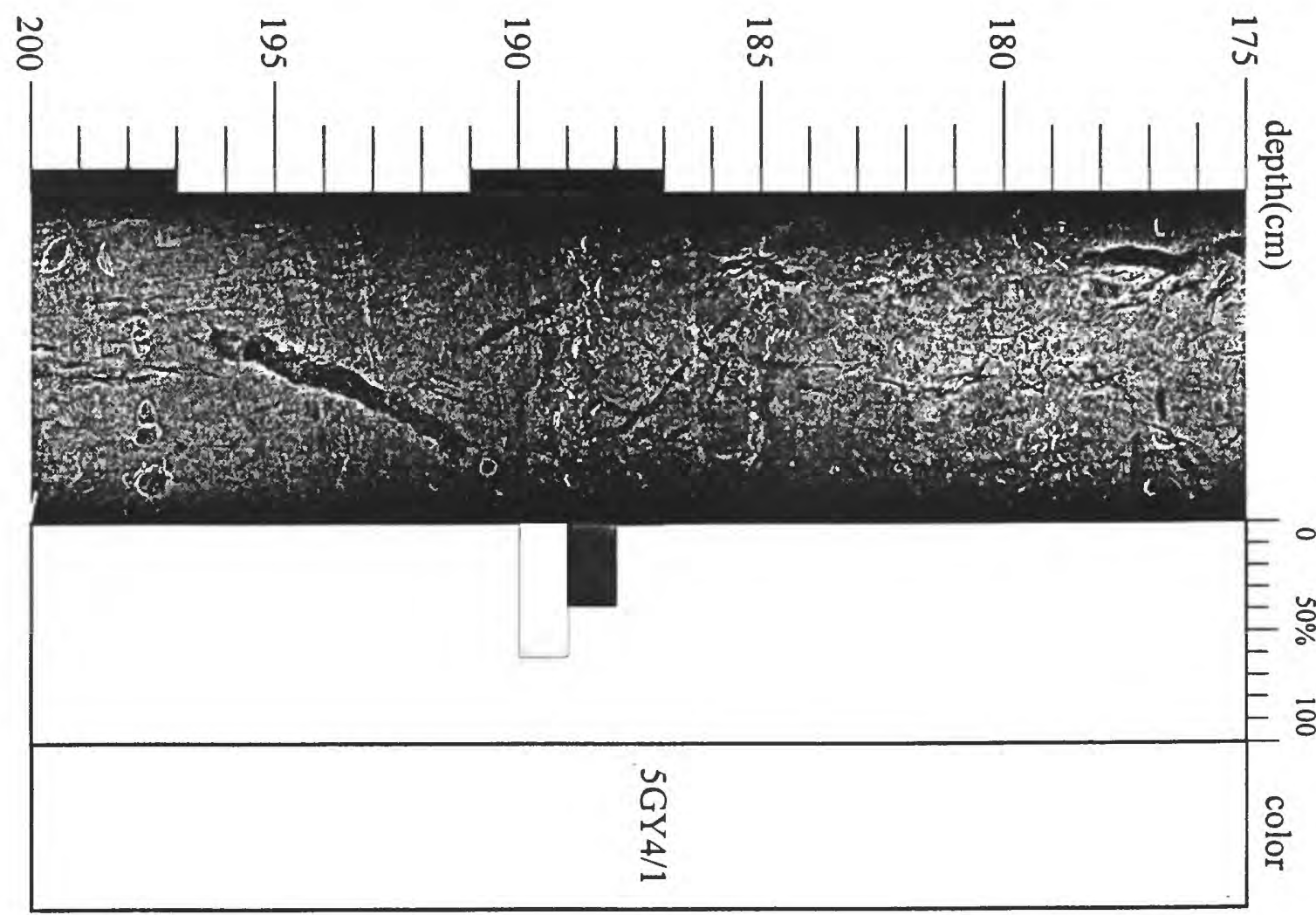

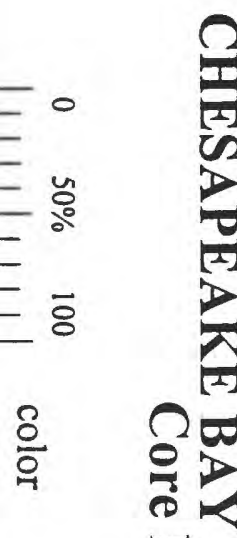

통

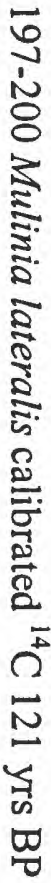

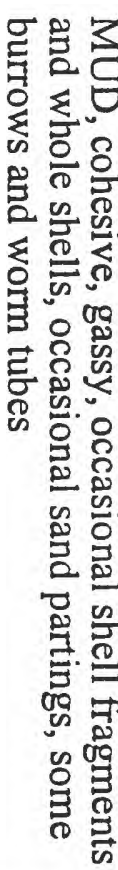




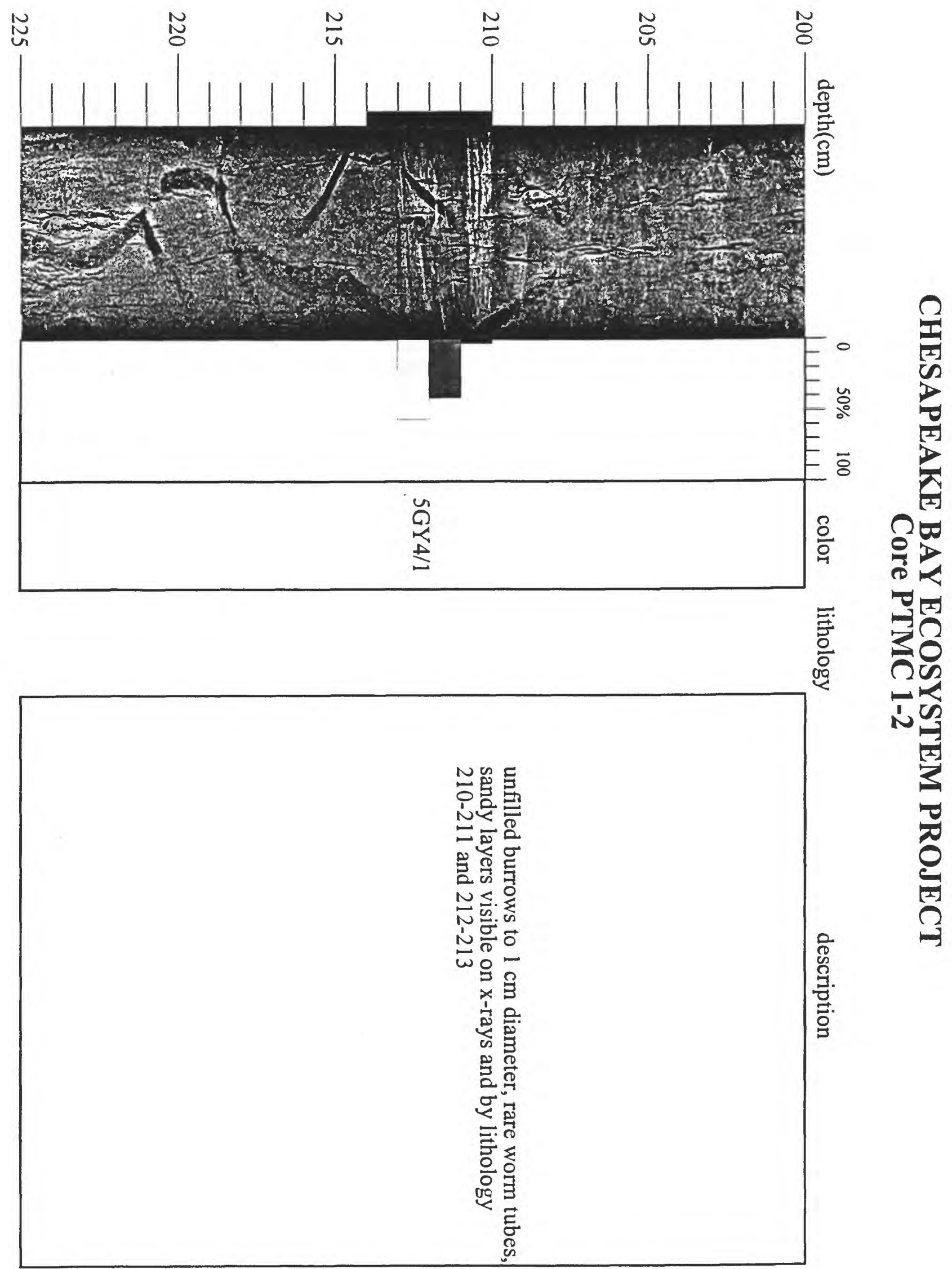




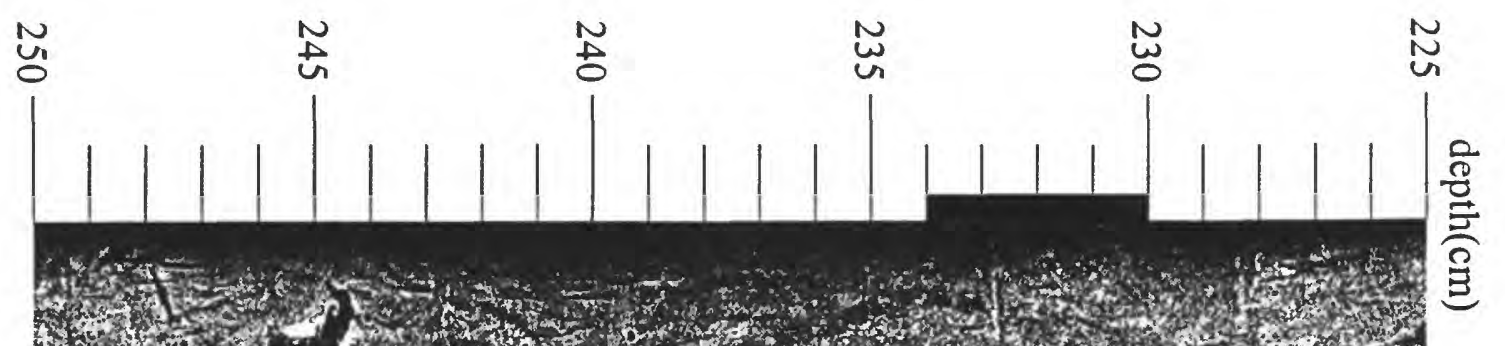

$\bar{G}$

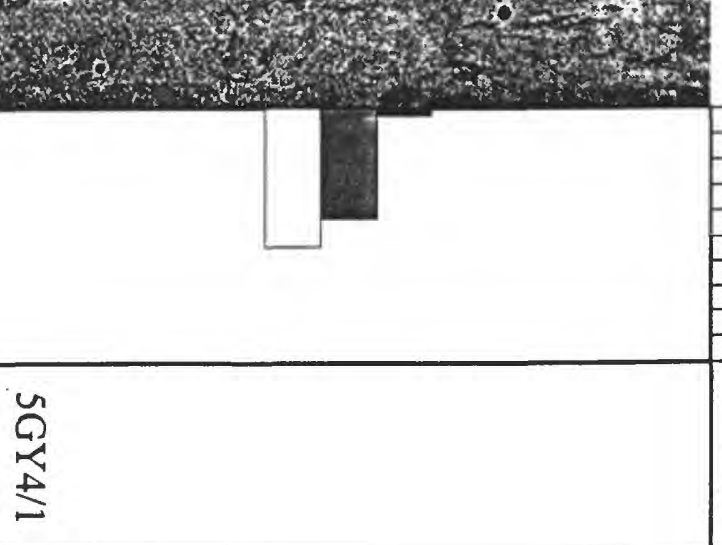

E.

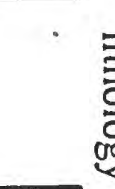

홍

마맘
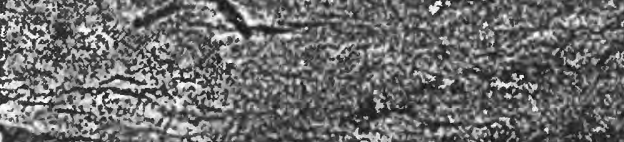

3 


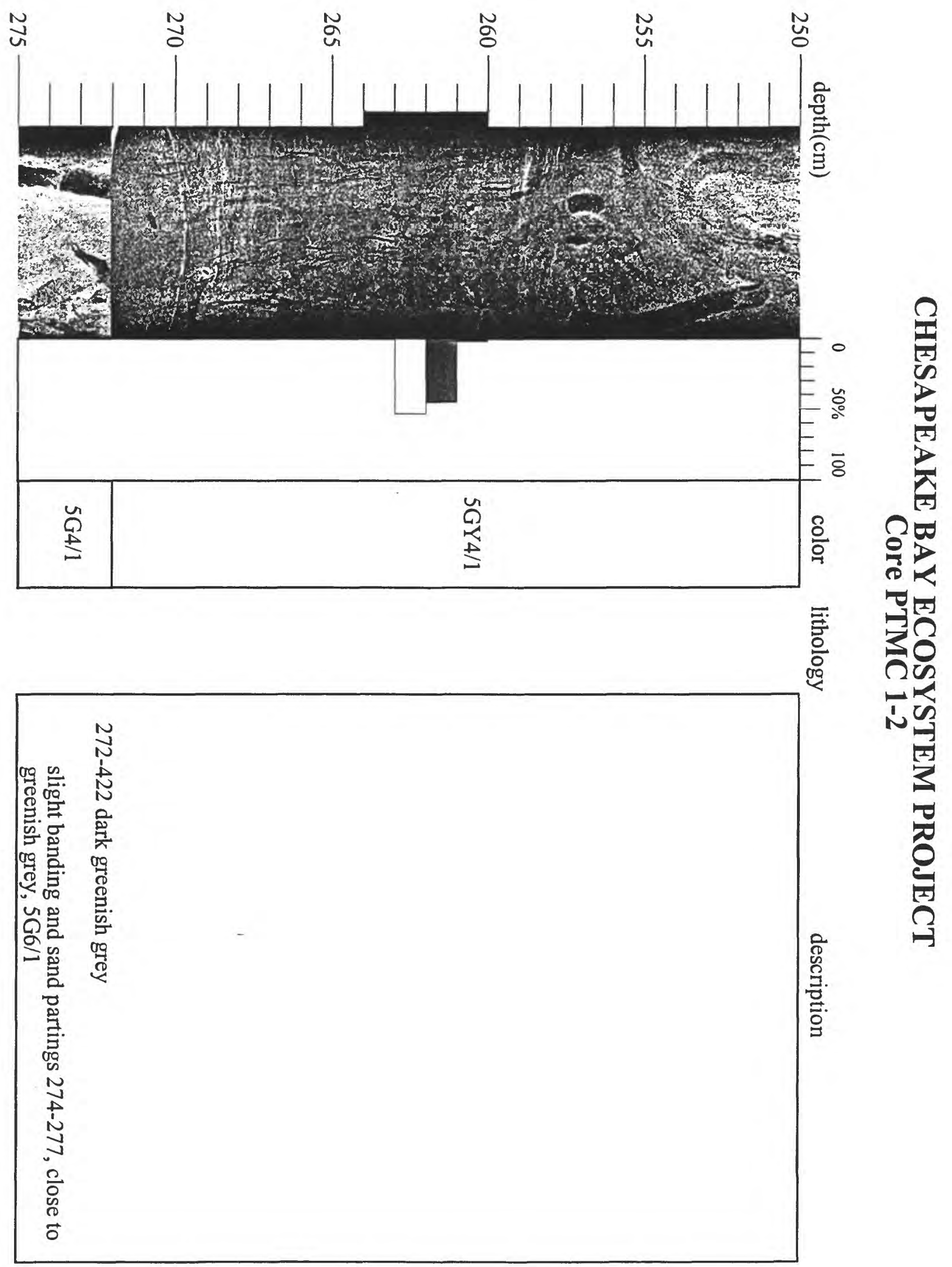


岁 芯 $\tilde{\square}$ 芯
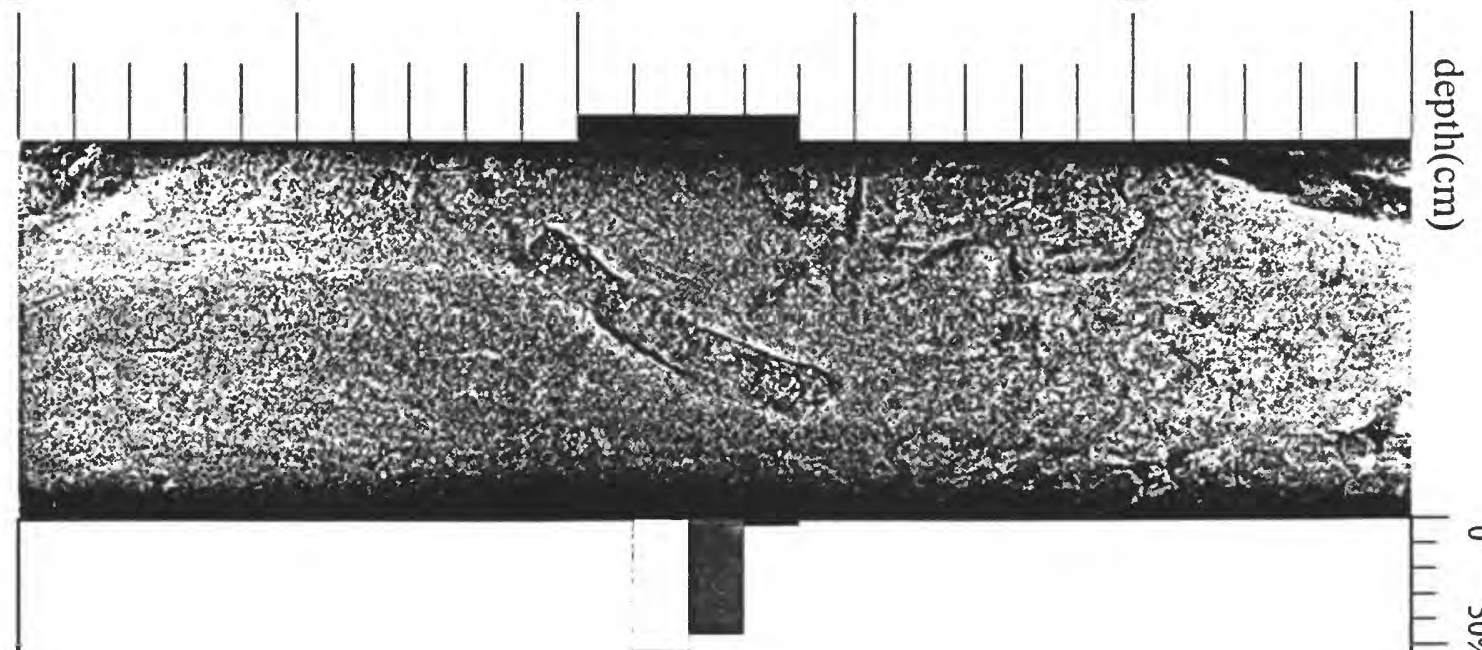

然

롱의

in

(1)

三N

$<\frac{2}{\sigma}$

品

$<\sum$

웅

政

힝응ㅇ

$\exists$

ㅇㅇㅇ

3

宛

党

总吉

$\cong$

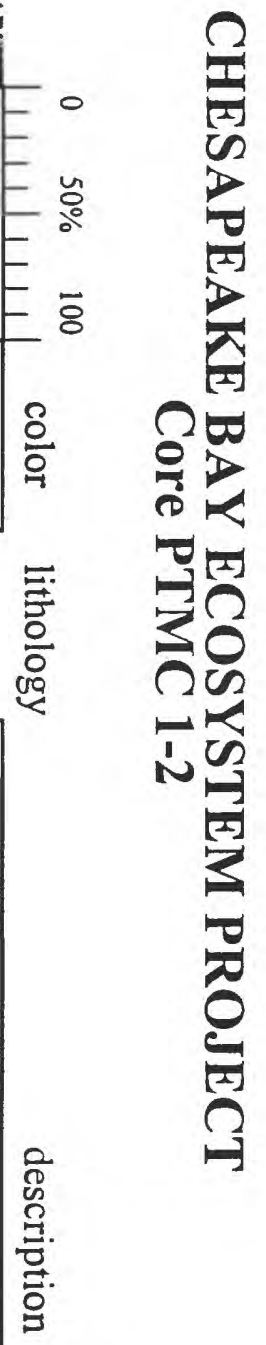




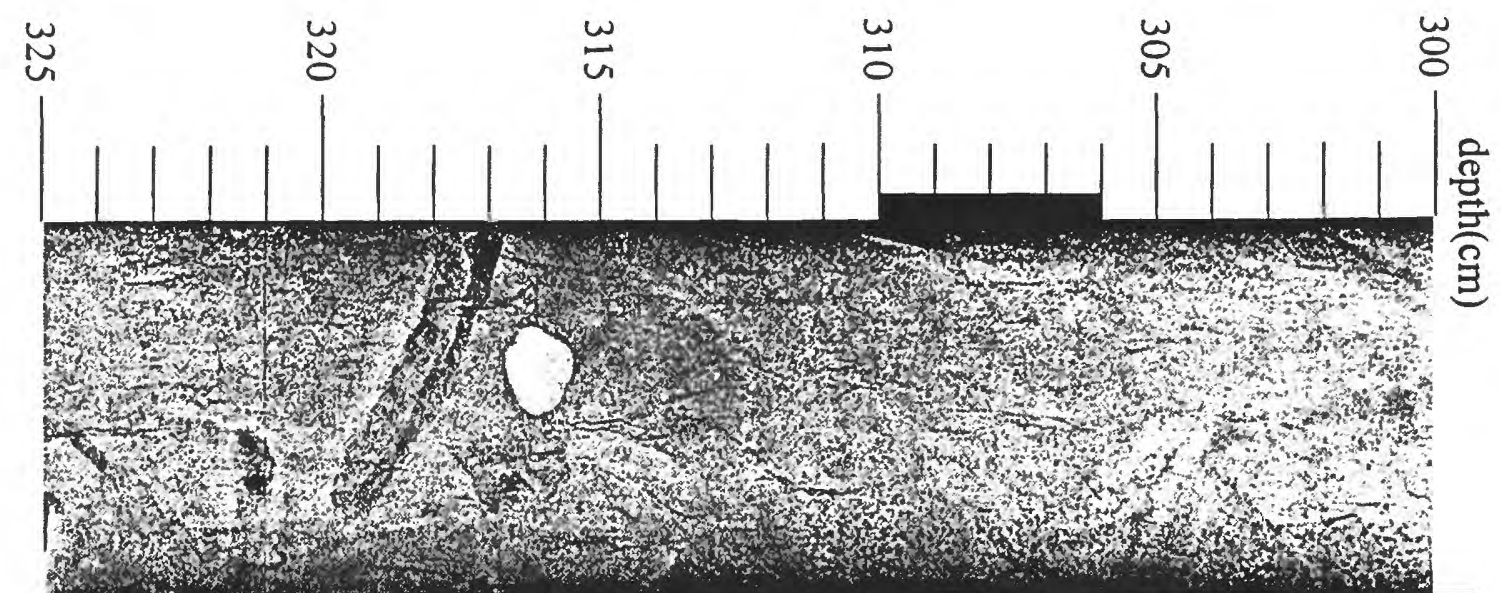

$\bar{a}$

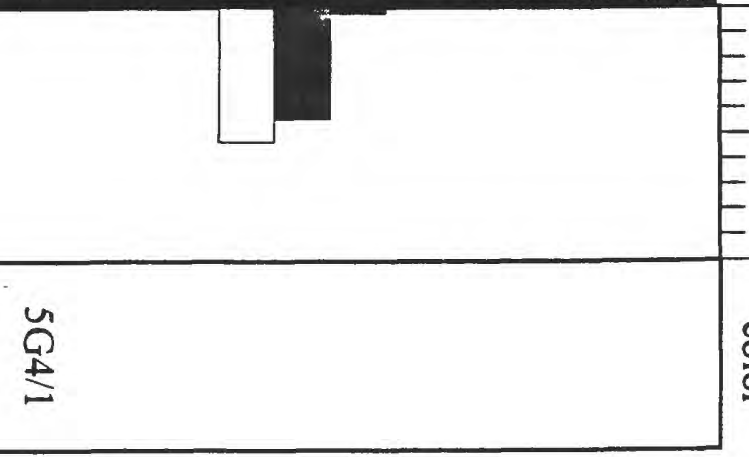

E。

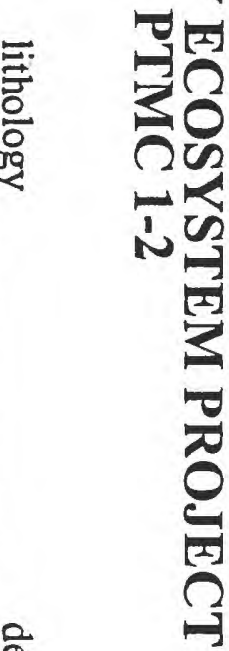

10

总

范.

苋 


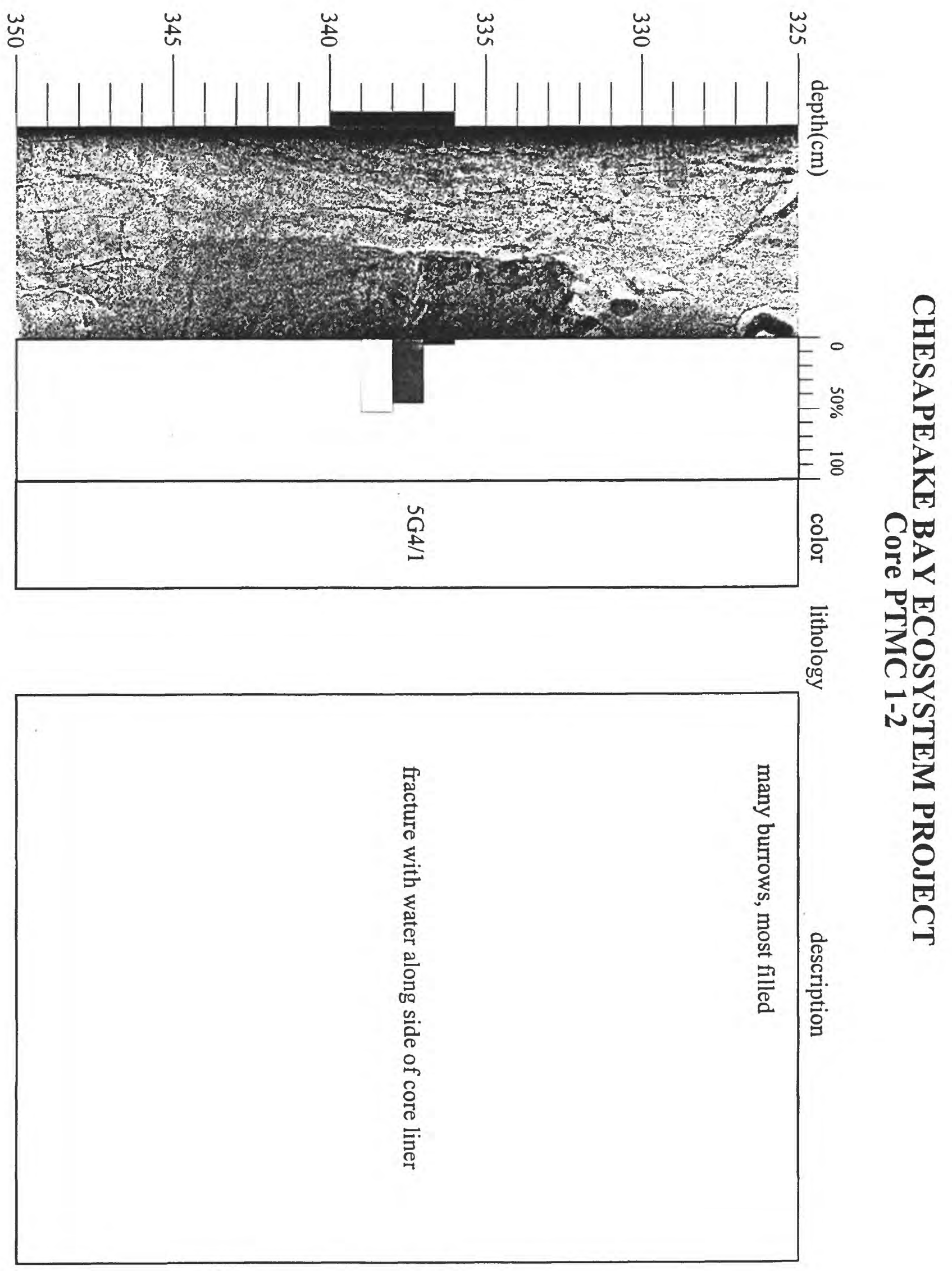



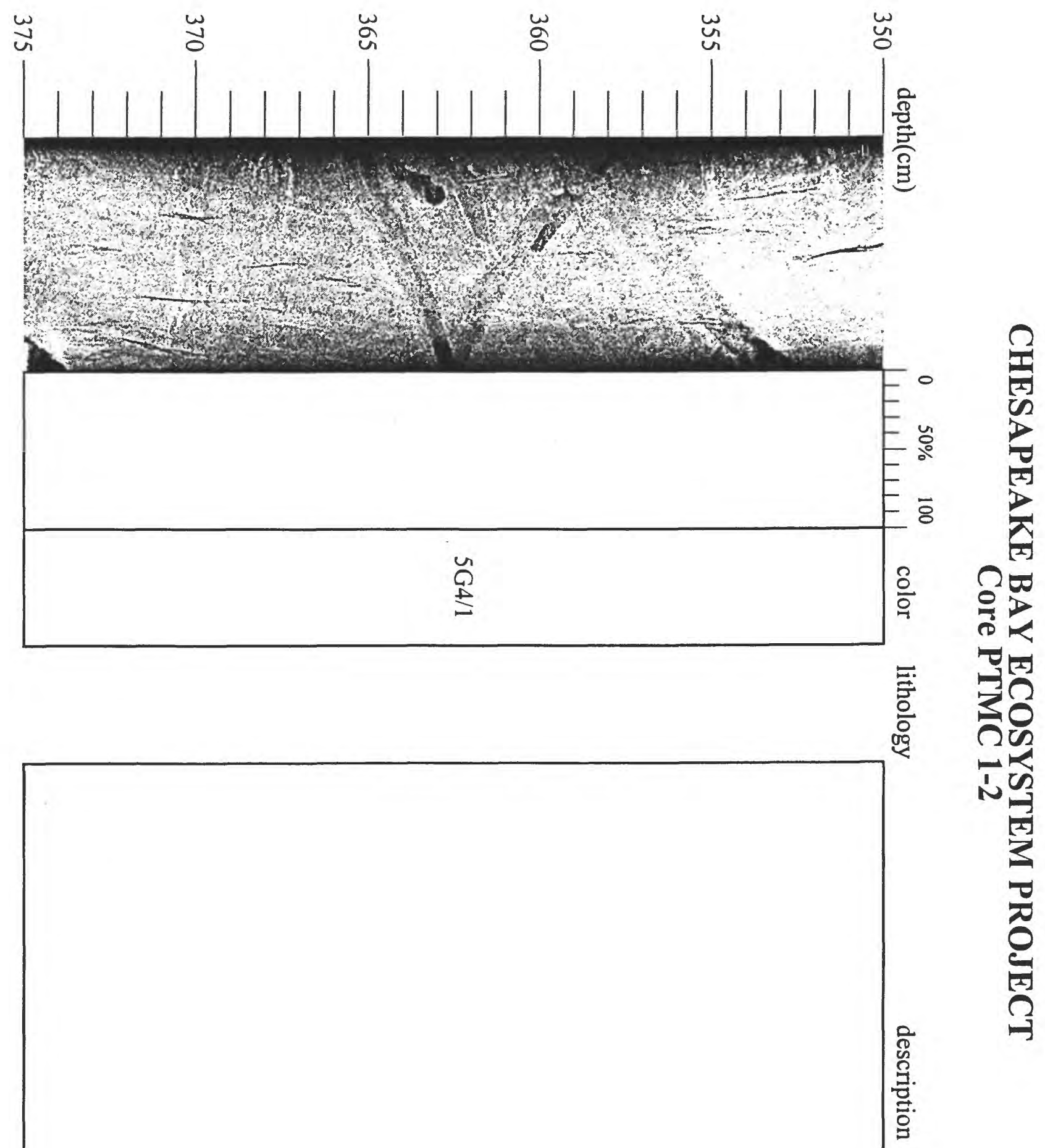


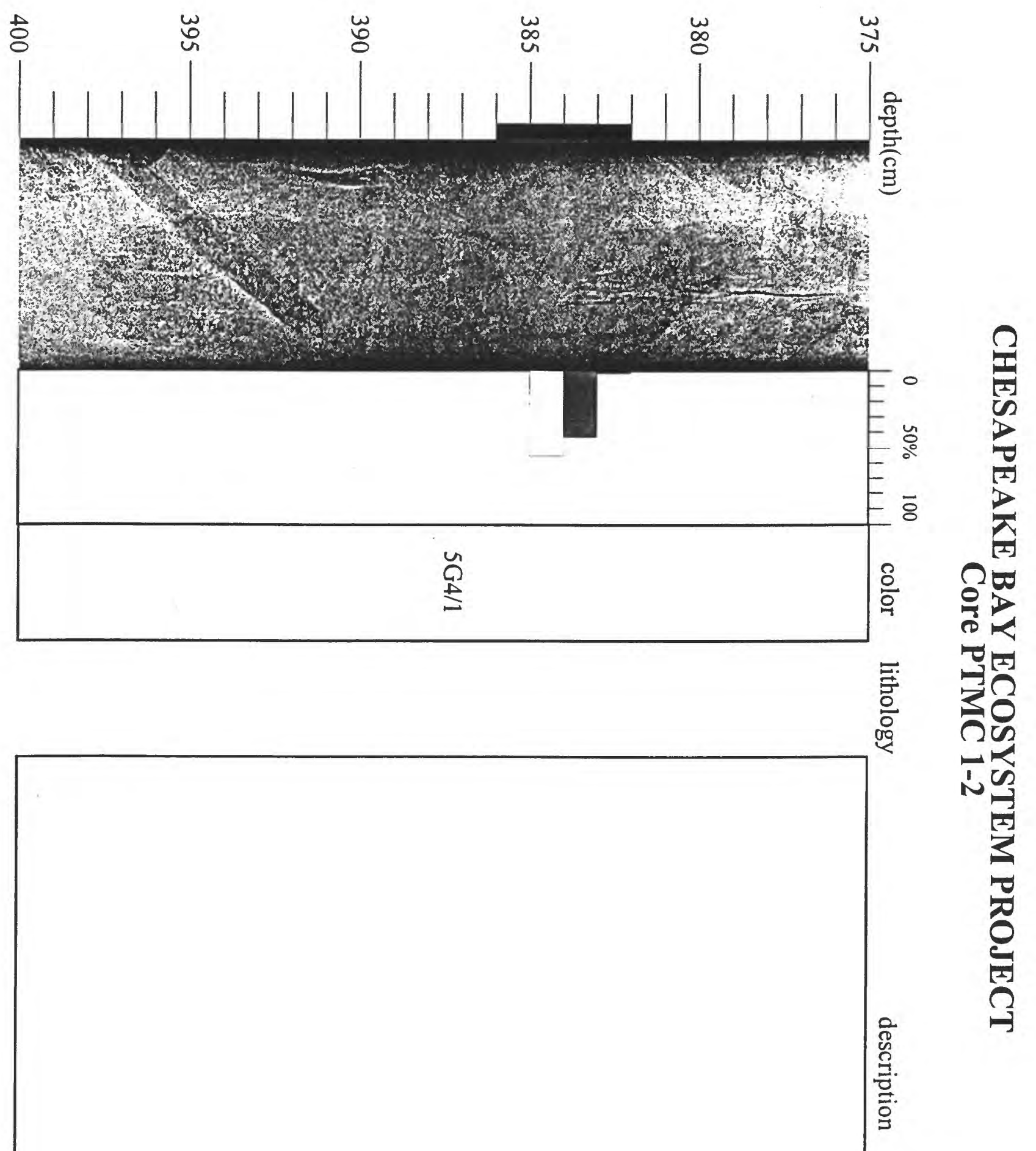




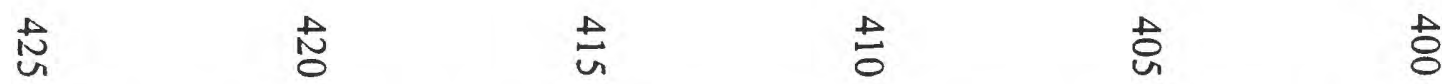

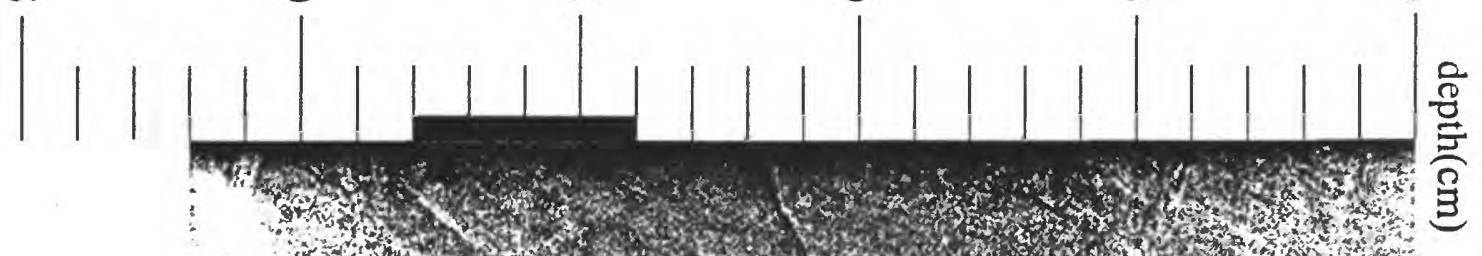

r.t.

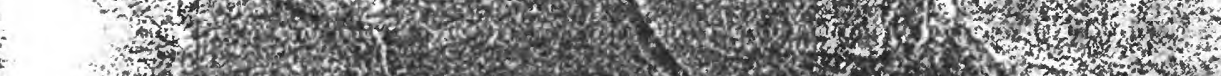

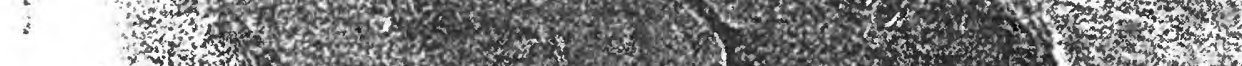

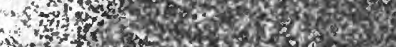

1.7.6.

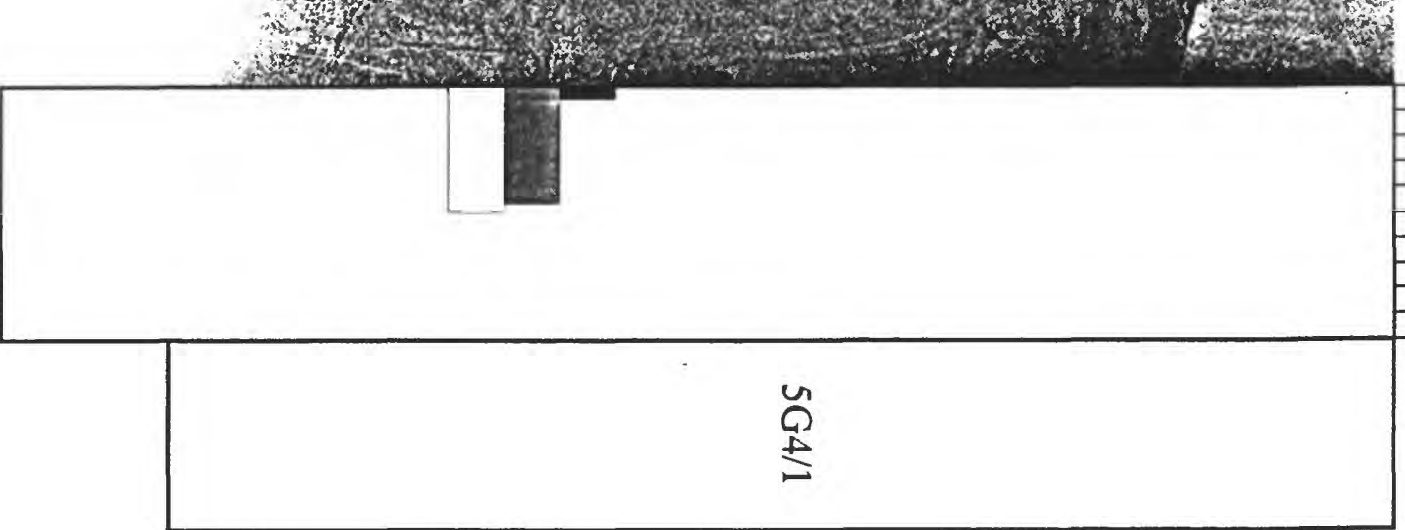

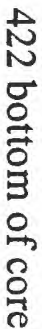

$=0$

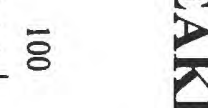

응 ?

王

0

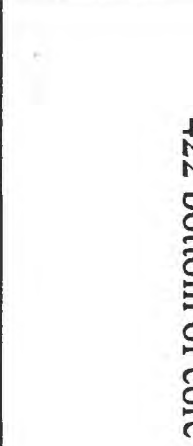

|20 


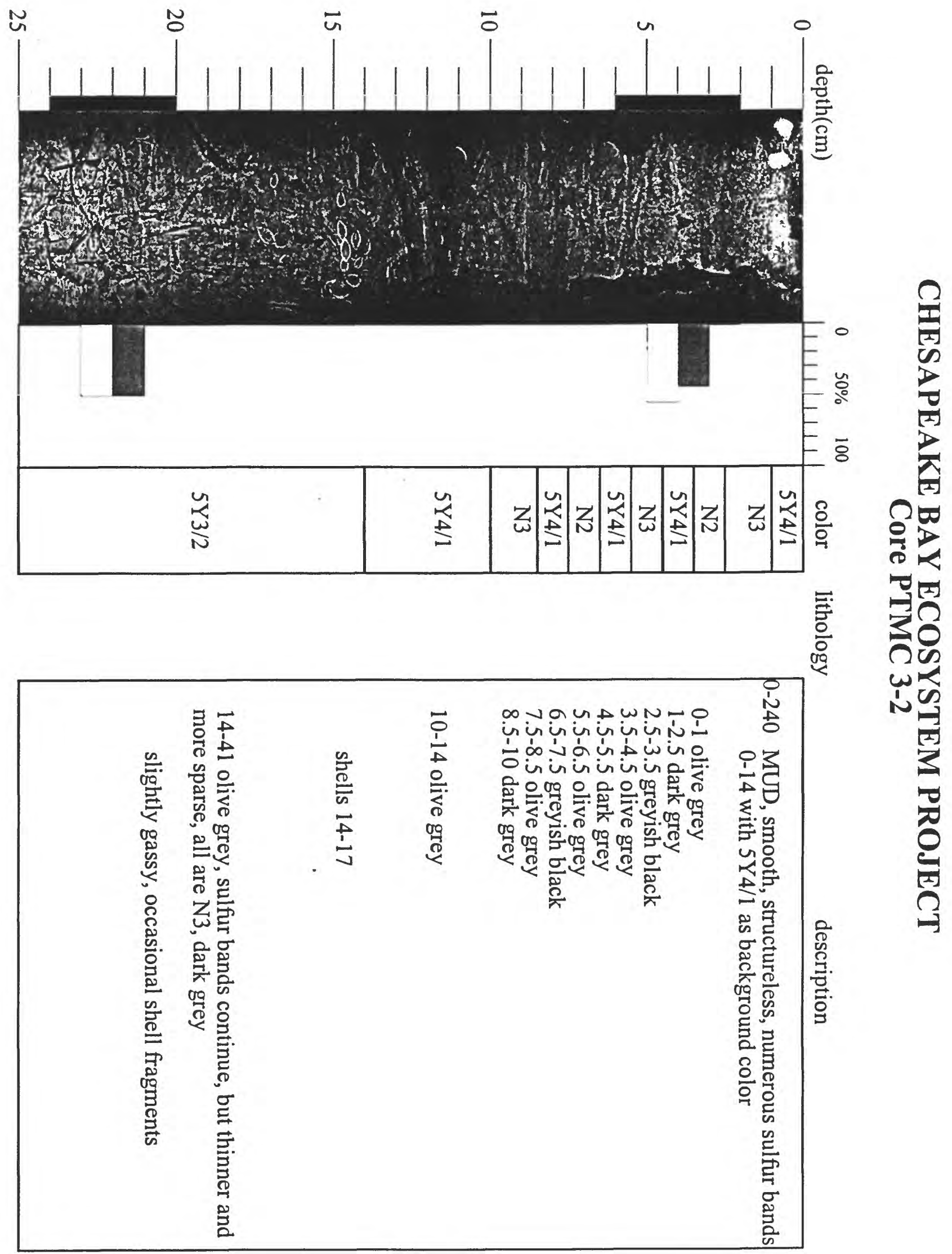




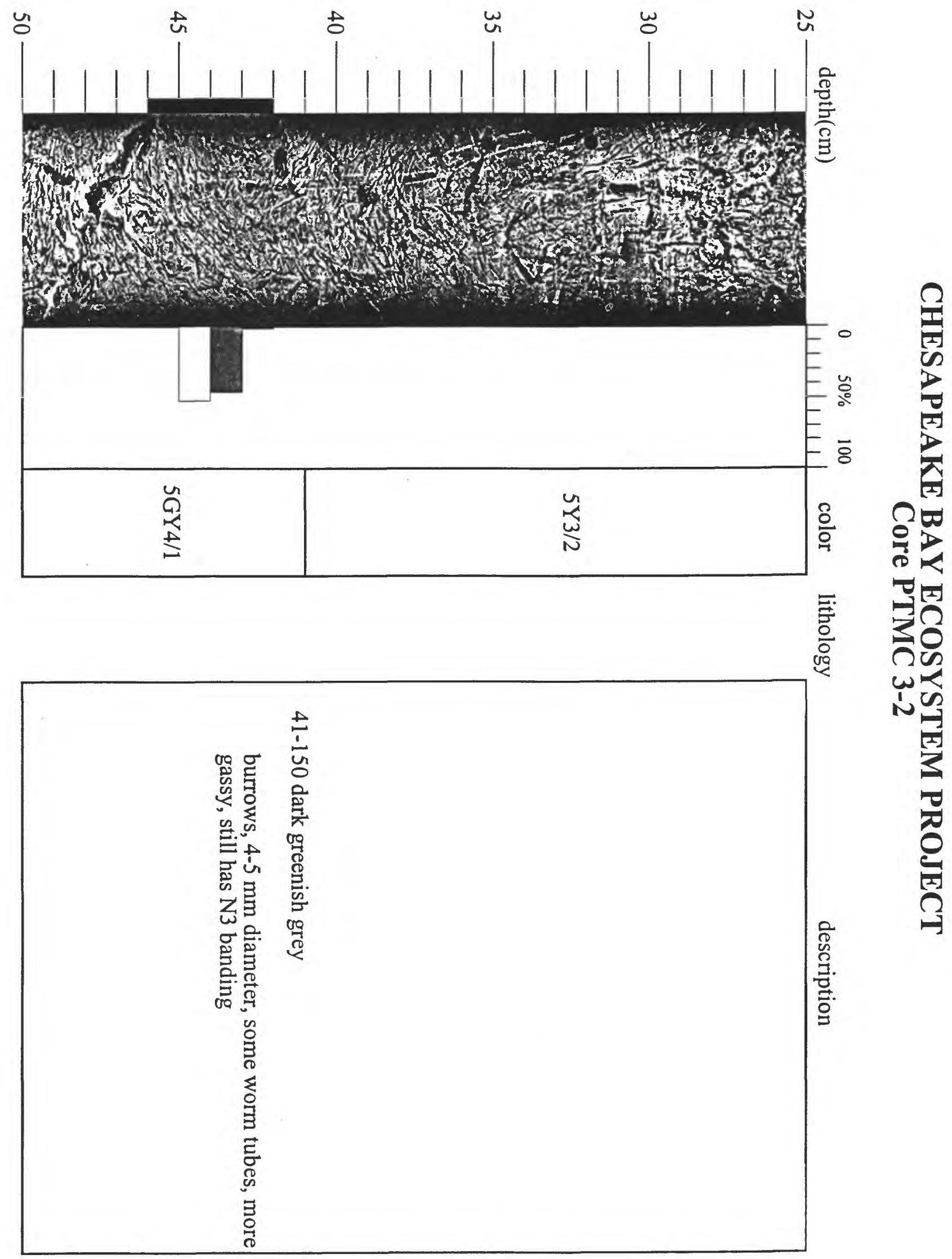




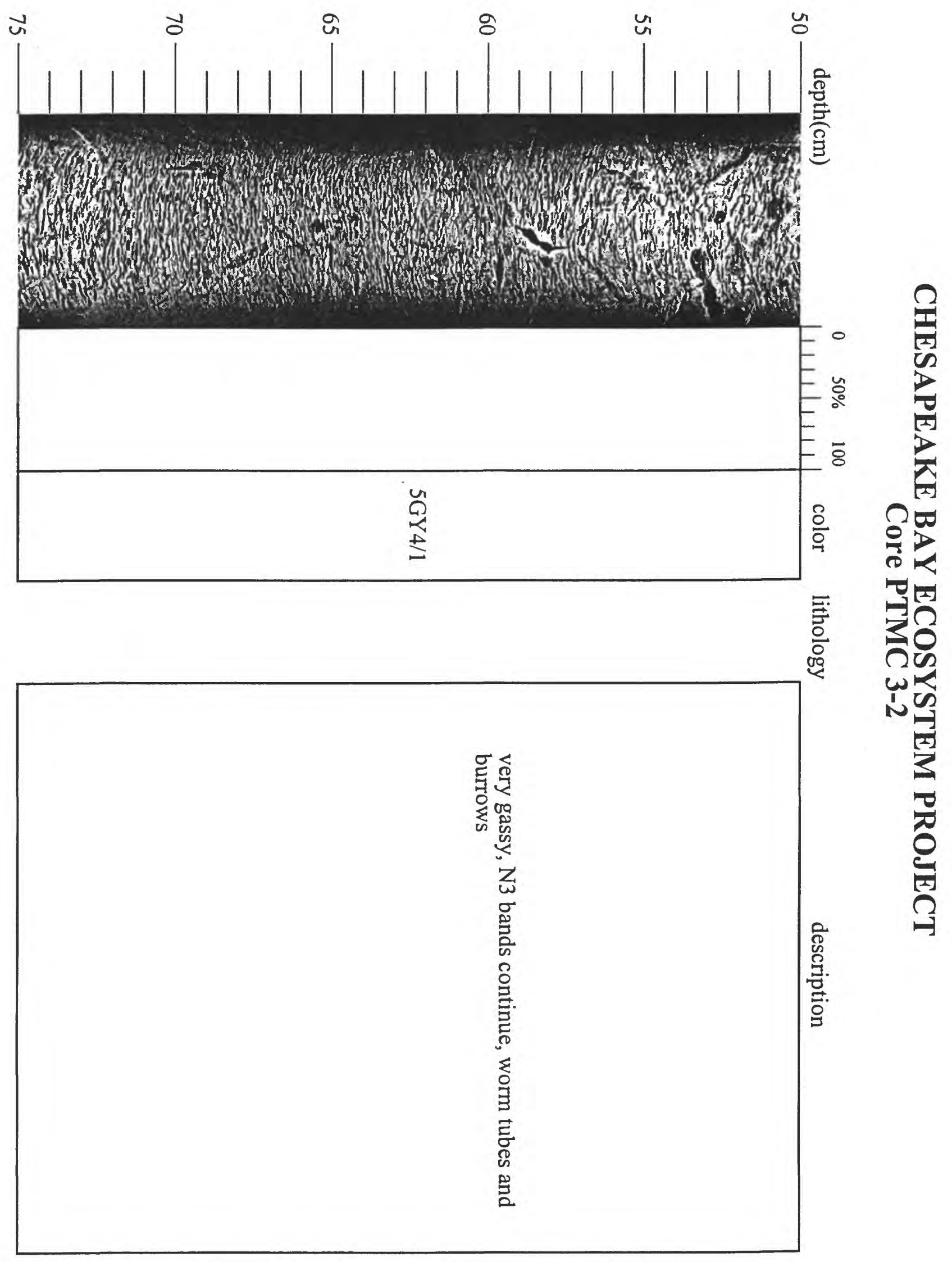



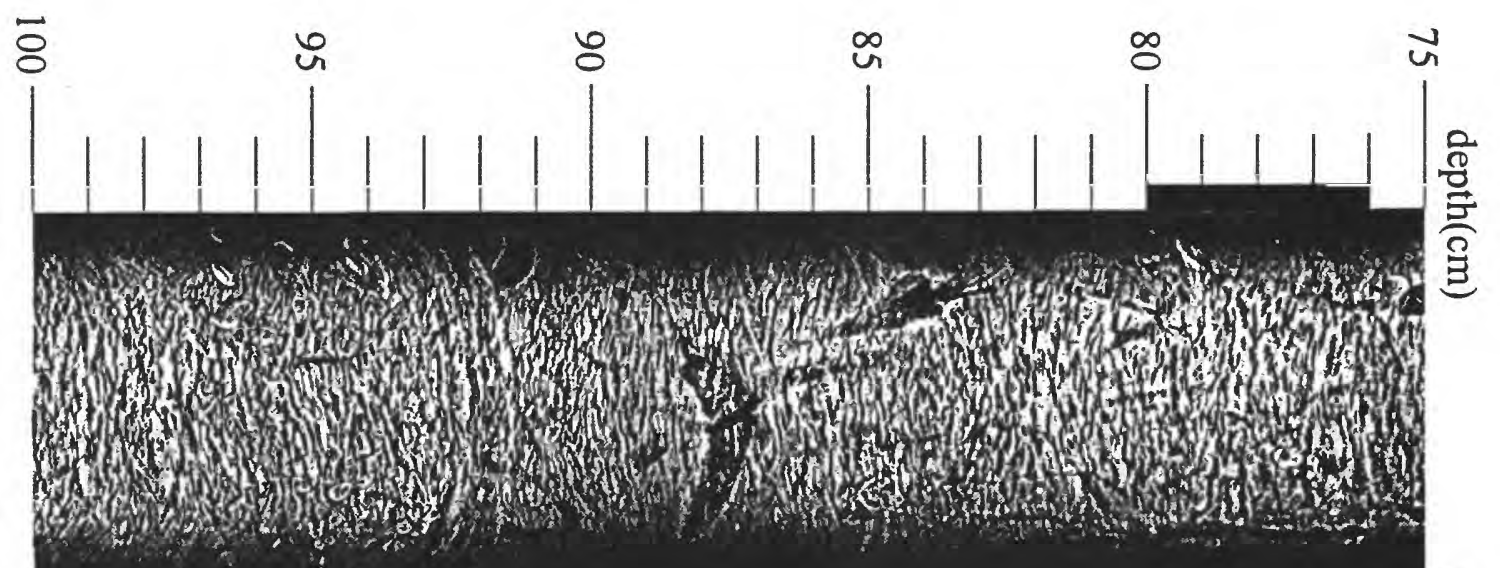

突

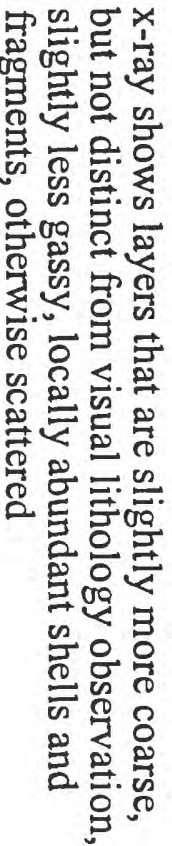

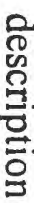

要 


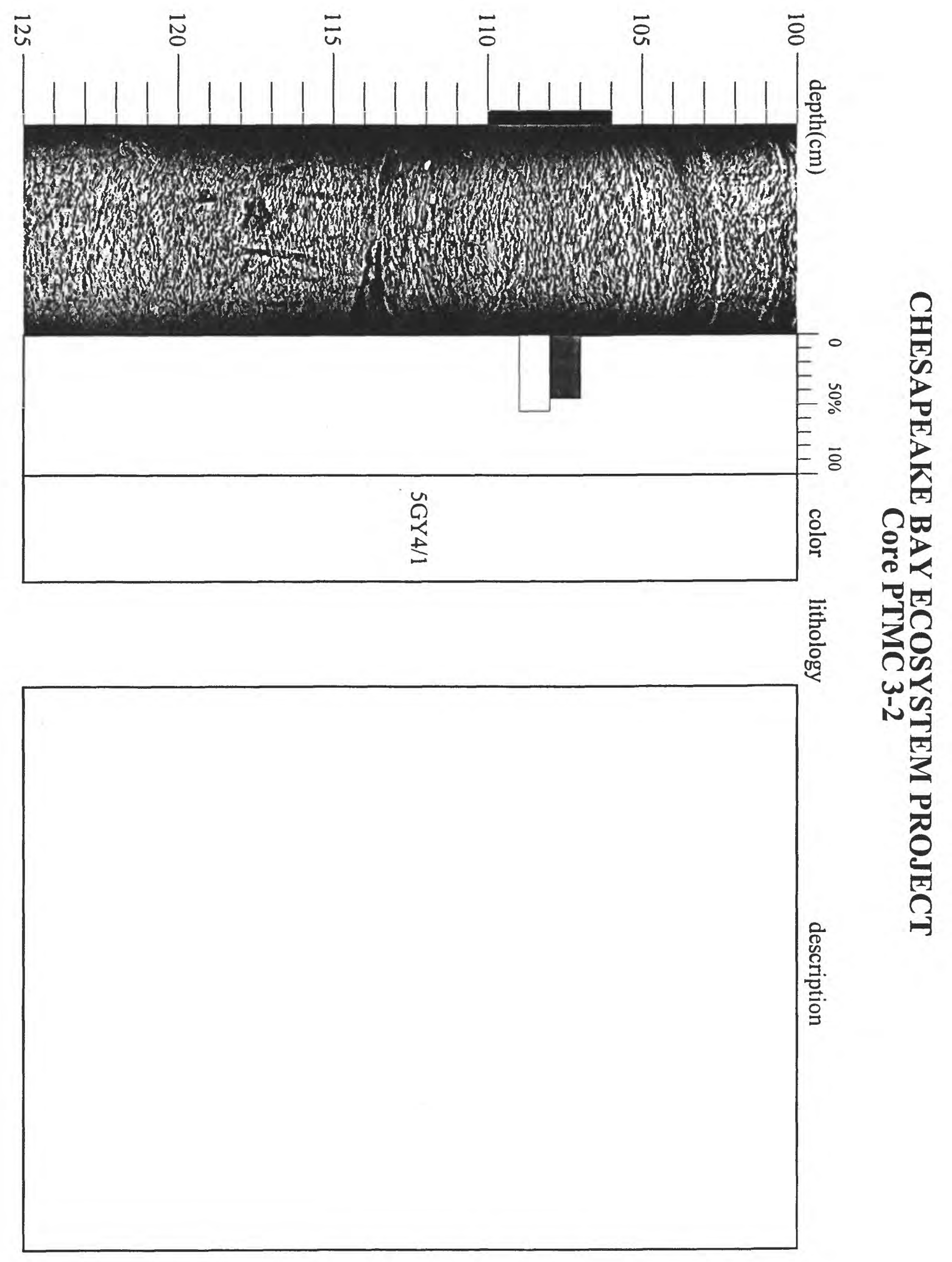




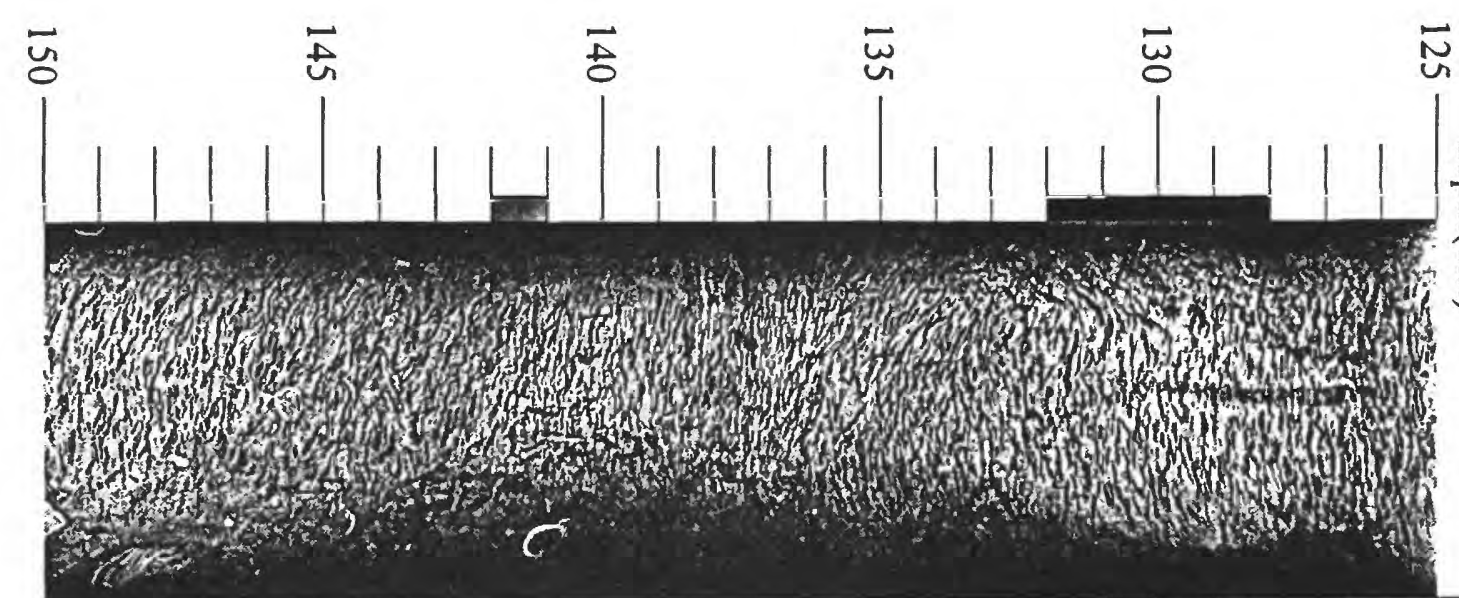

章

$=0$

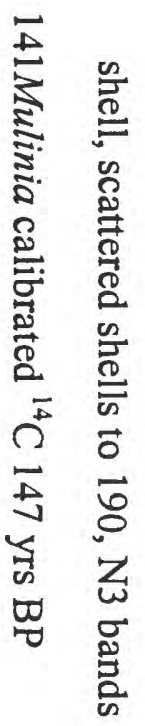

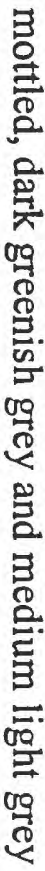

言 


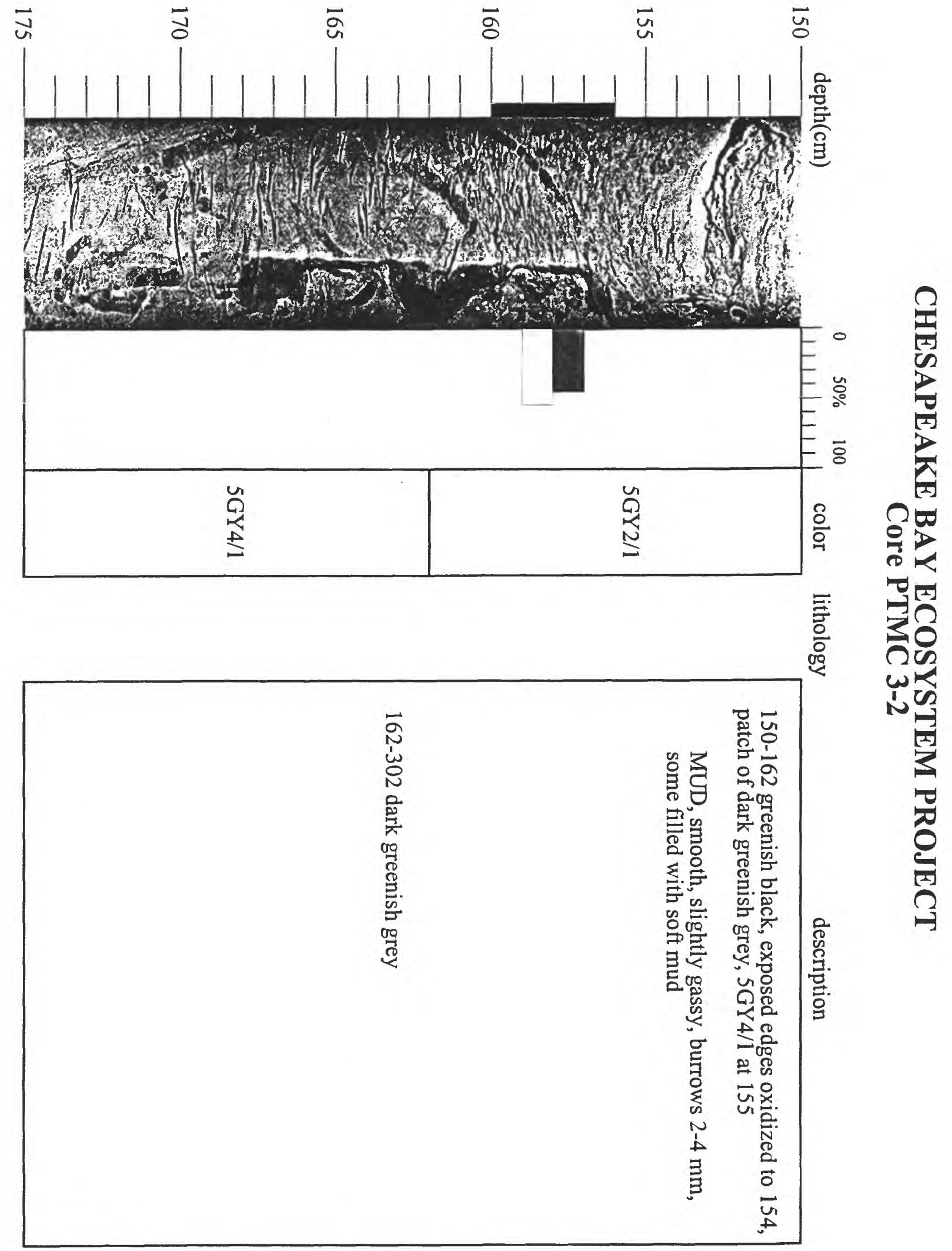




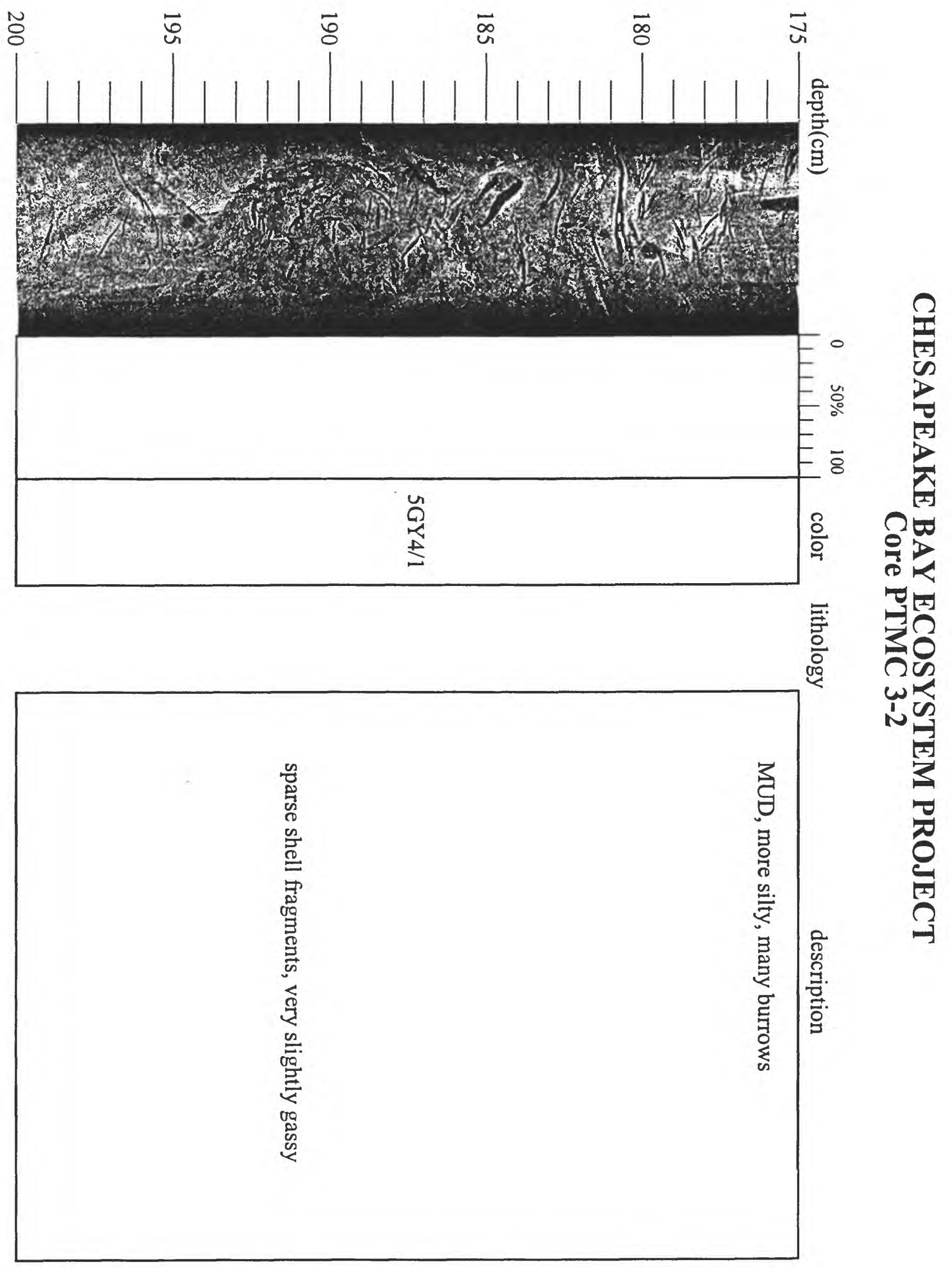




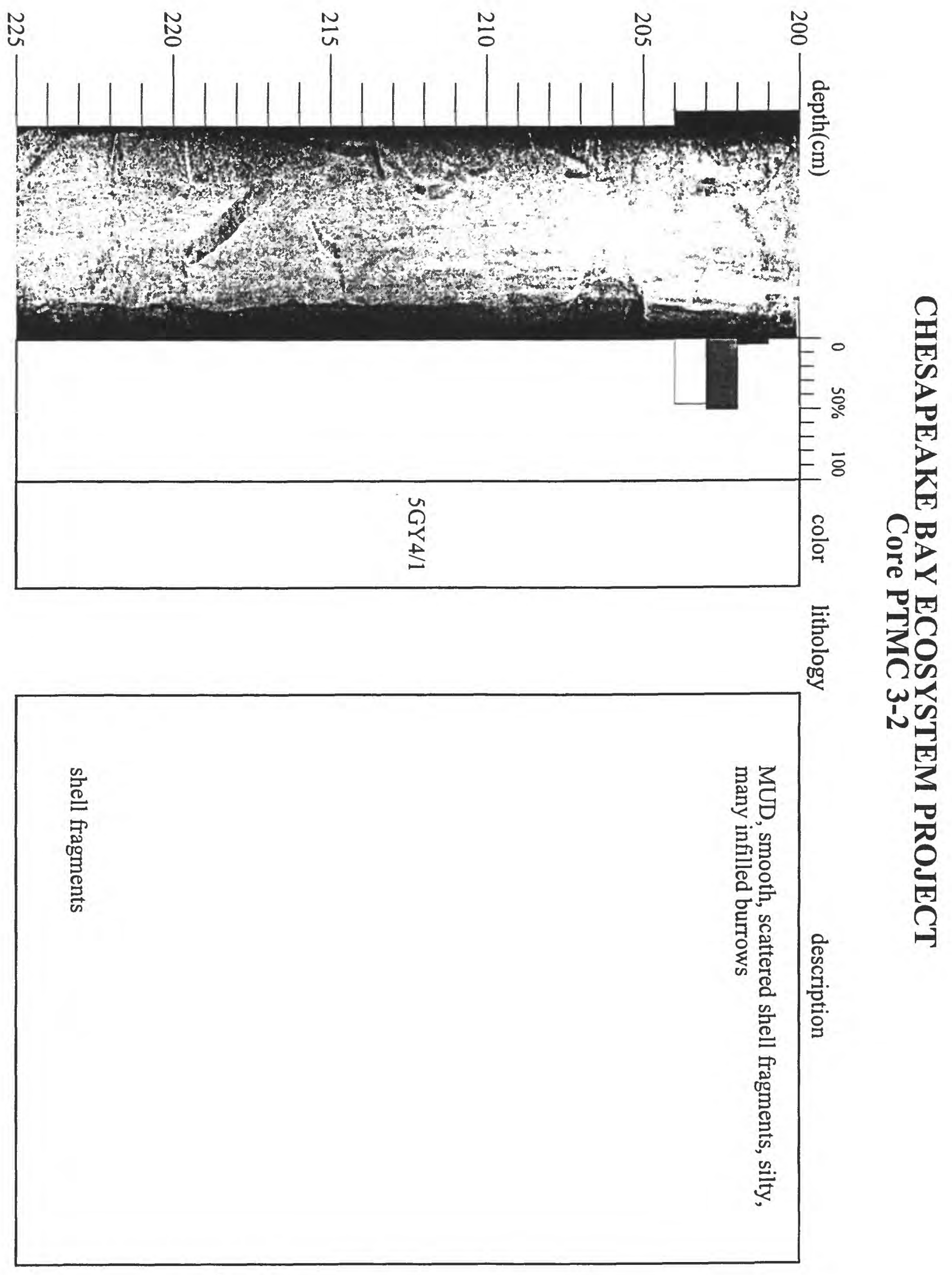



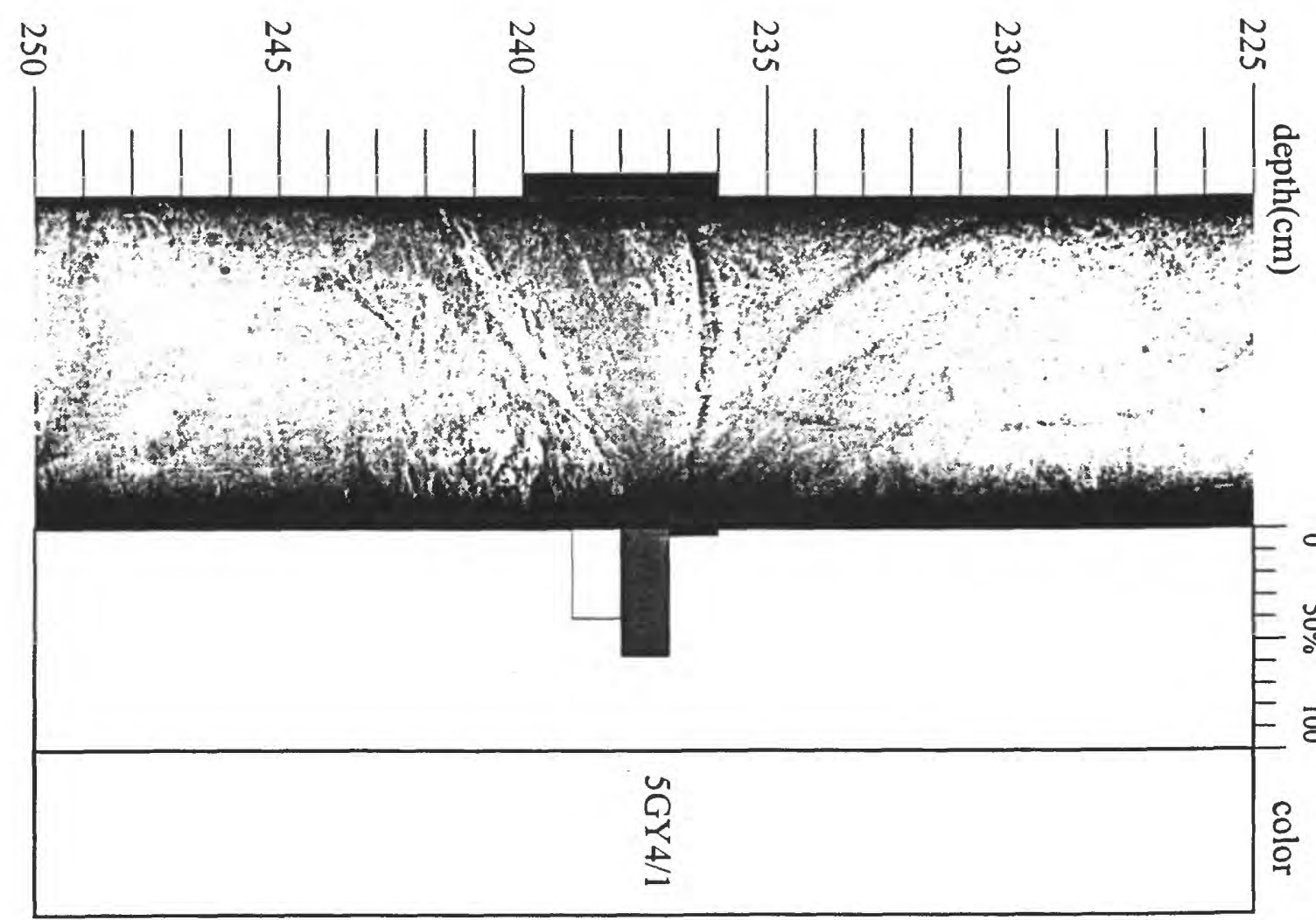

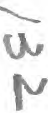

豆

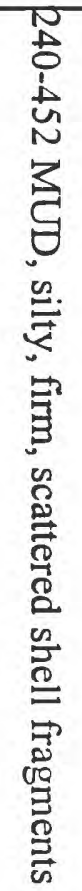

告

$<$

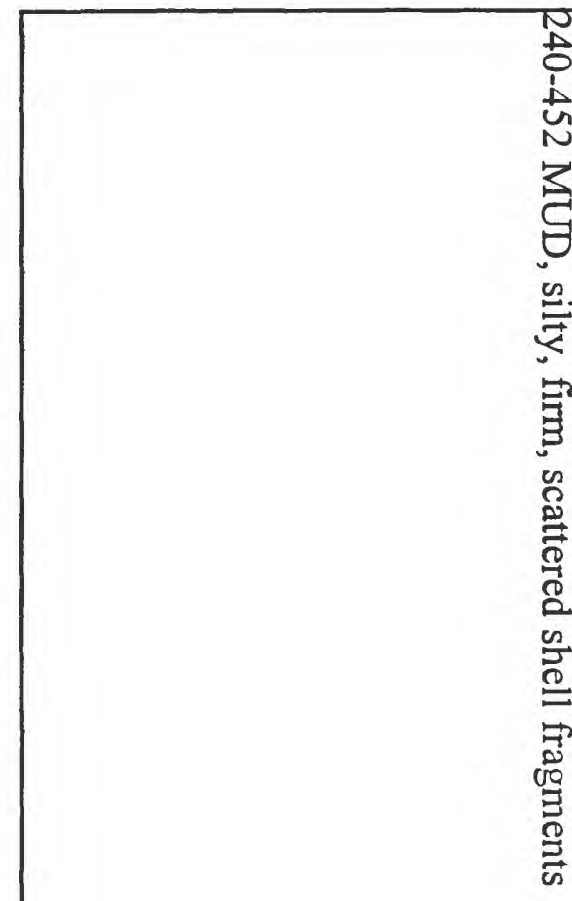



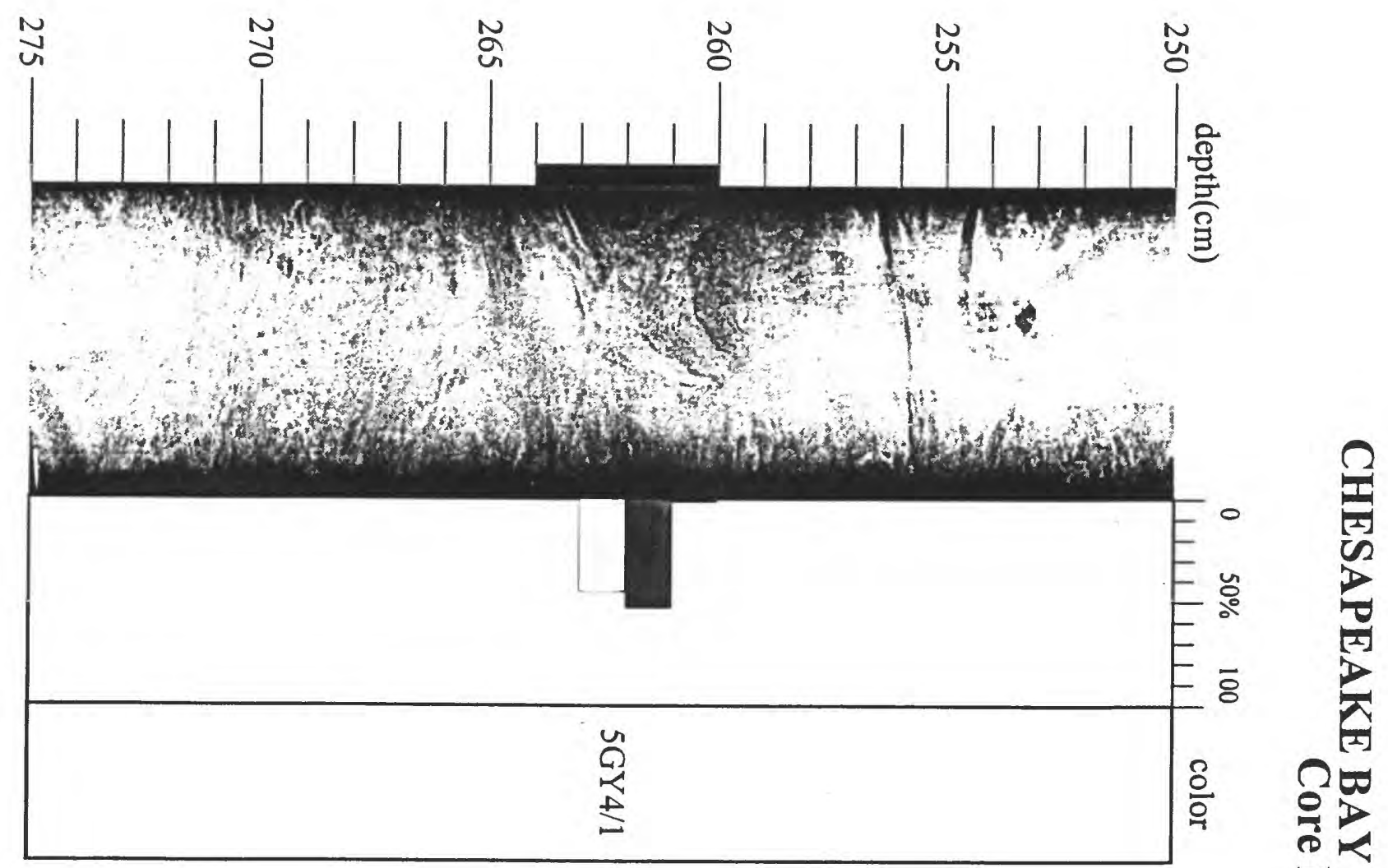

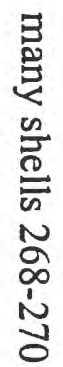

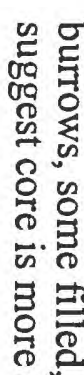

鱼次

的察

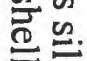

吾高

(स)

号 

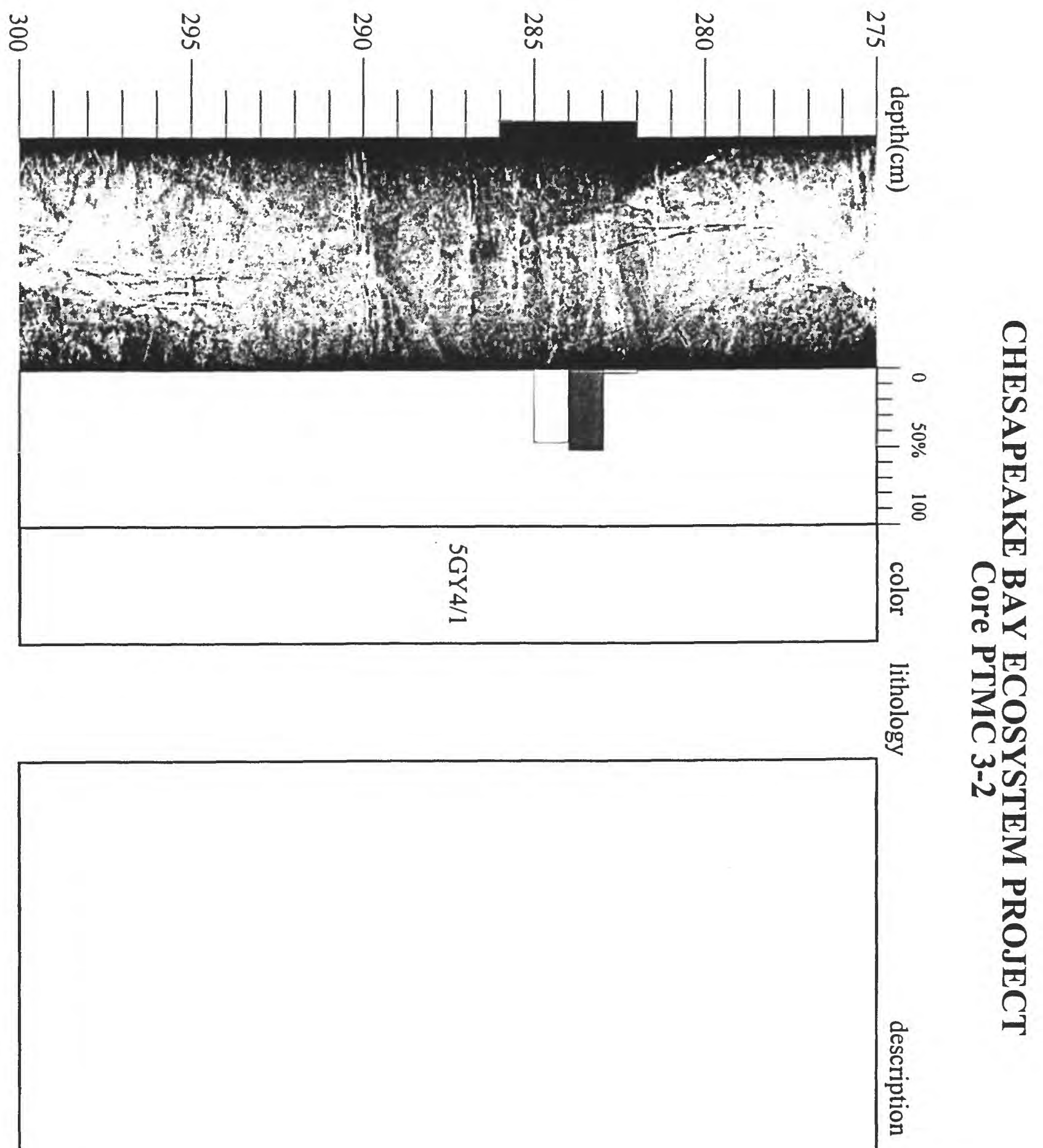


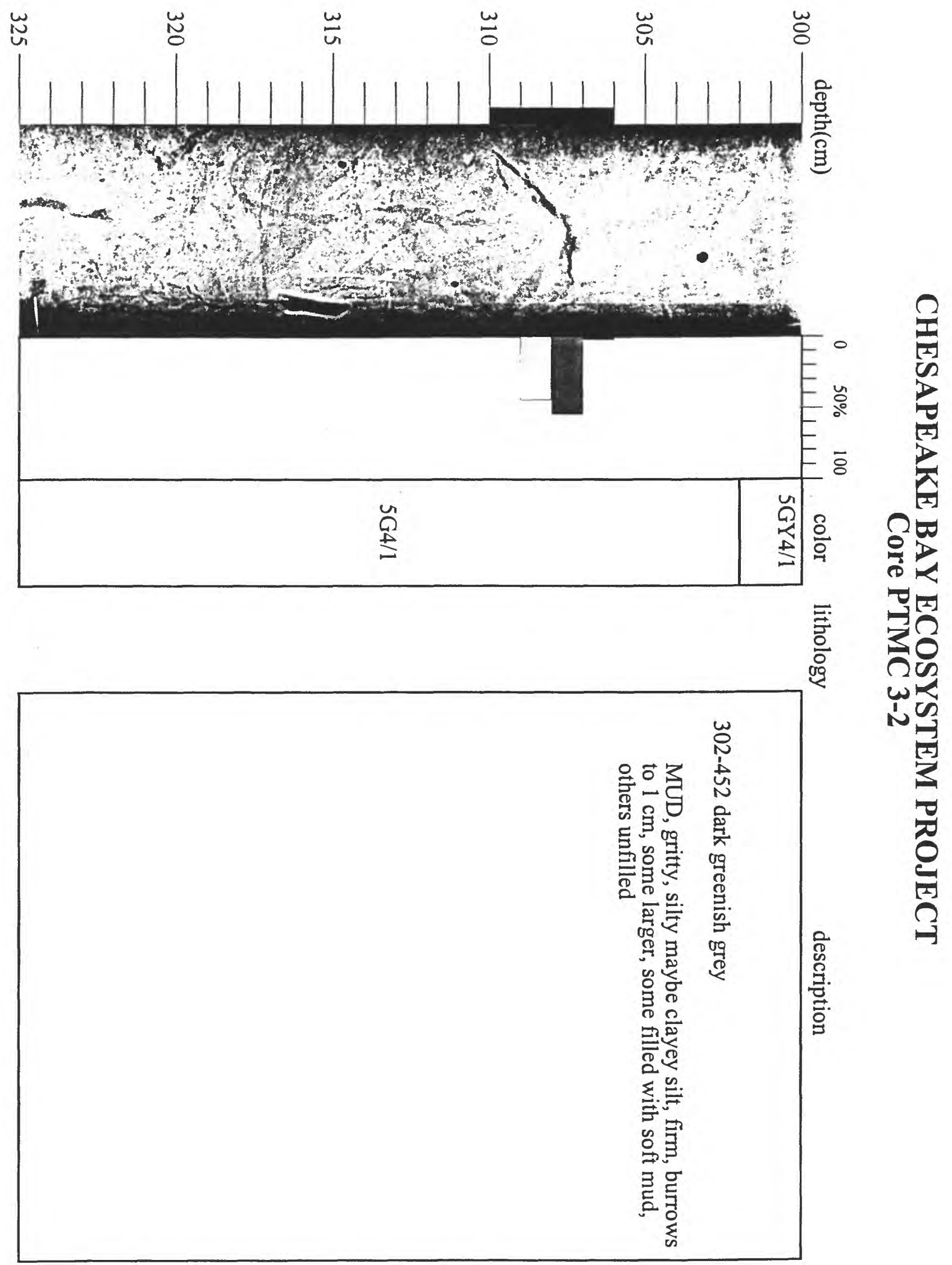



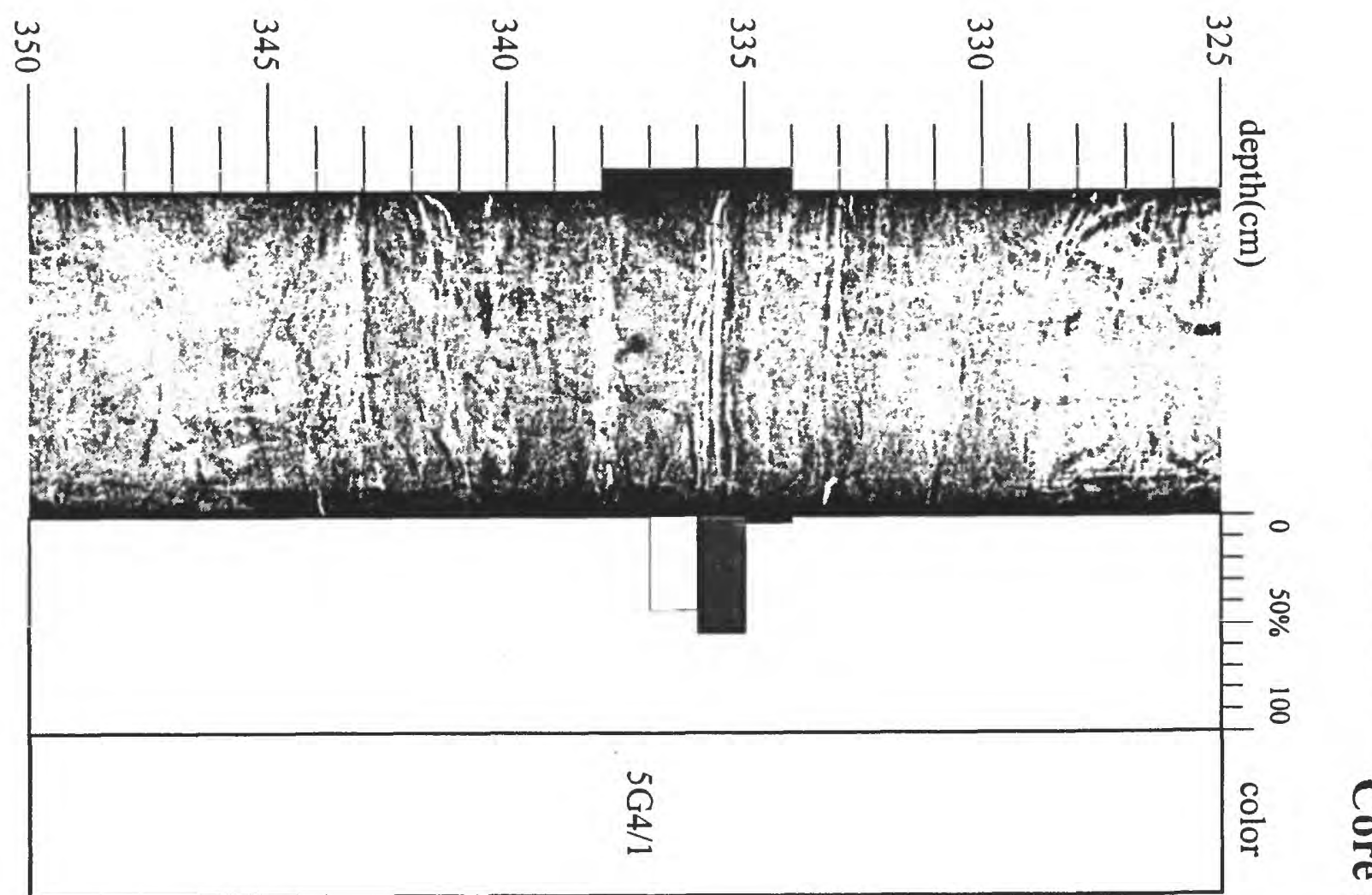



言 

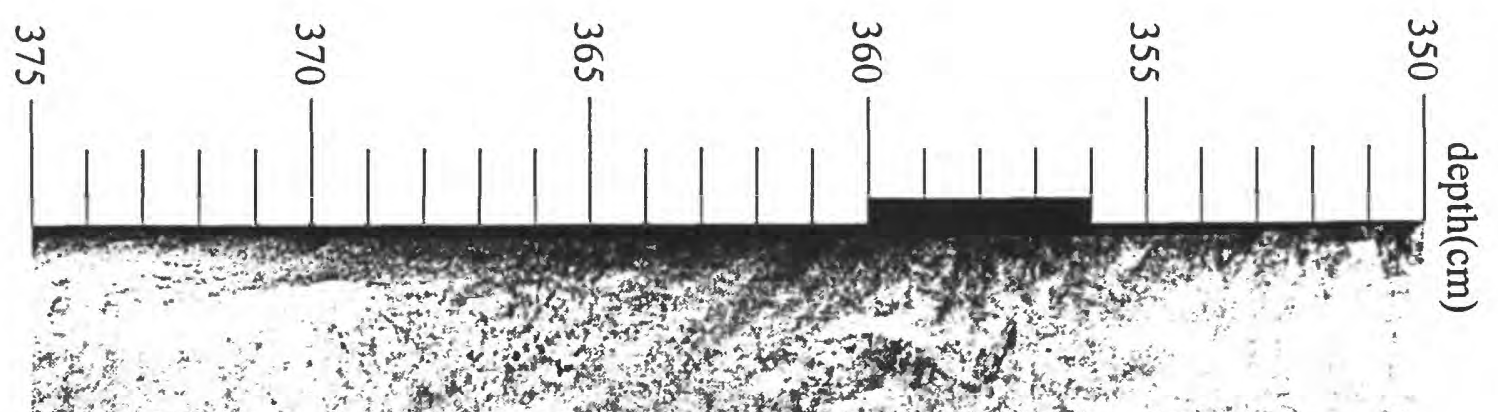

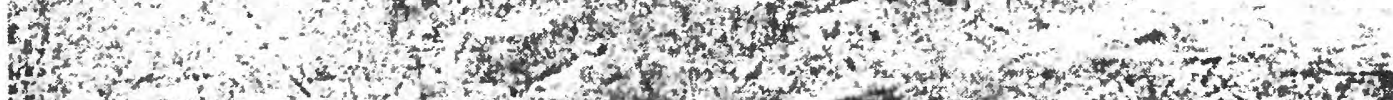
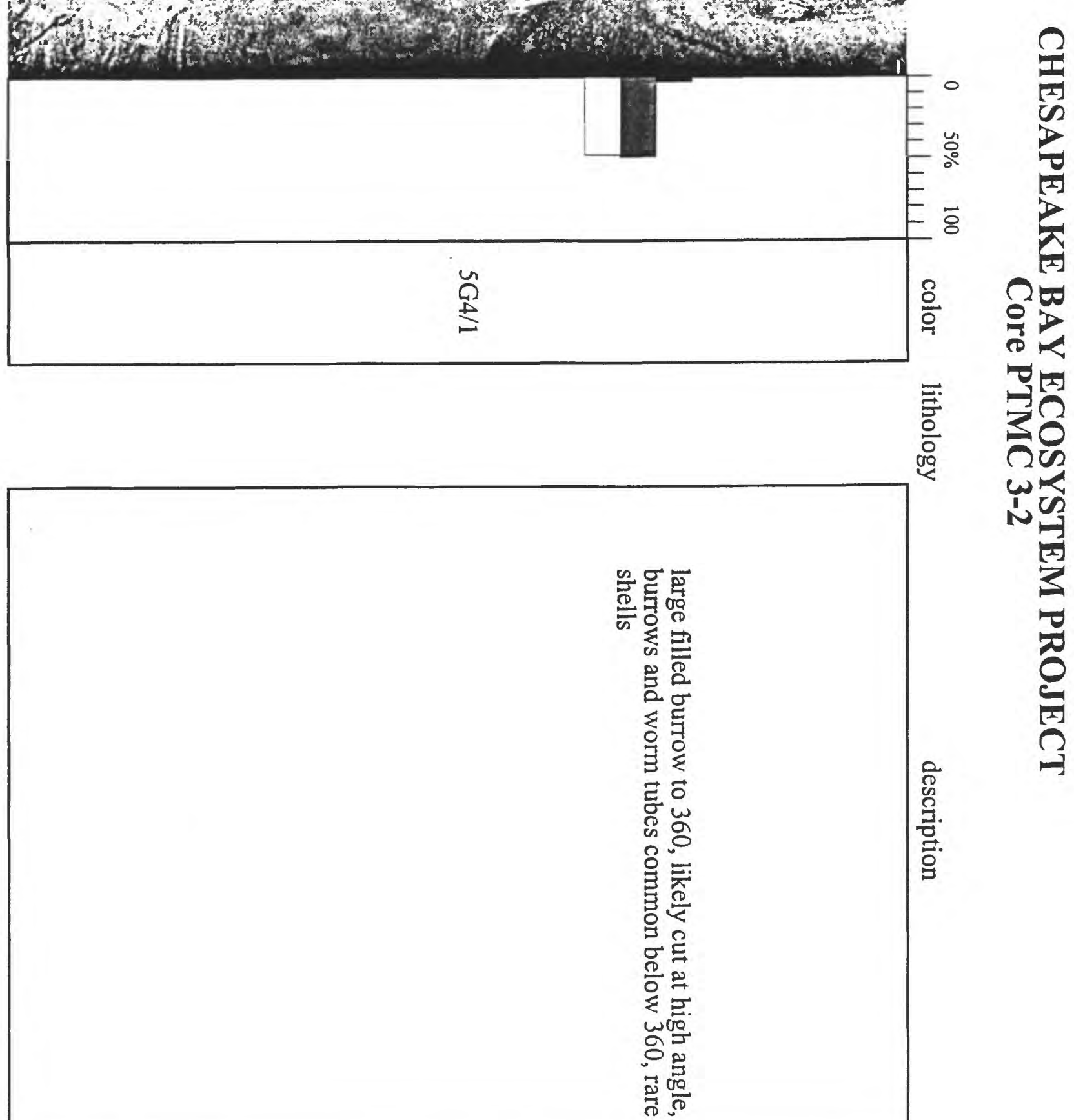


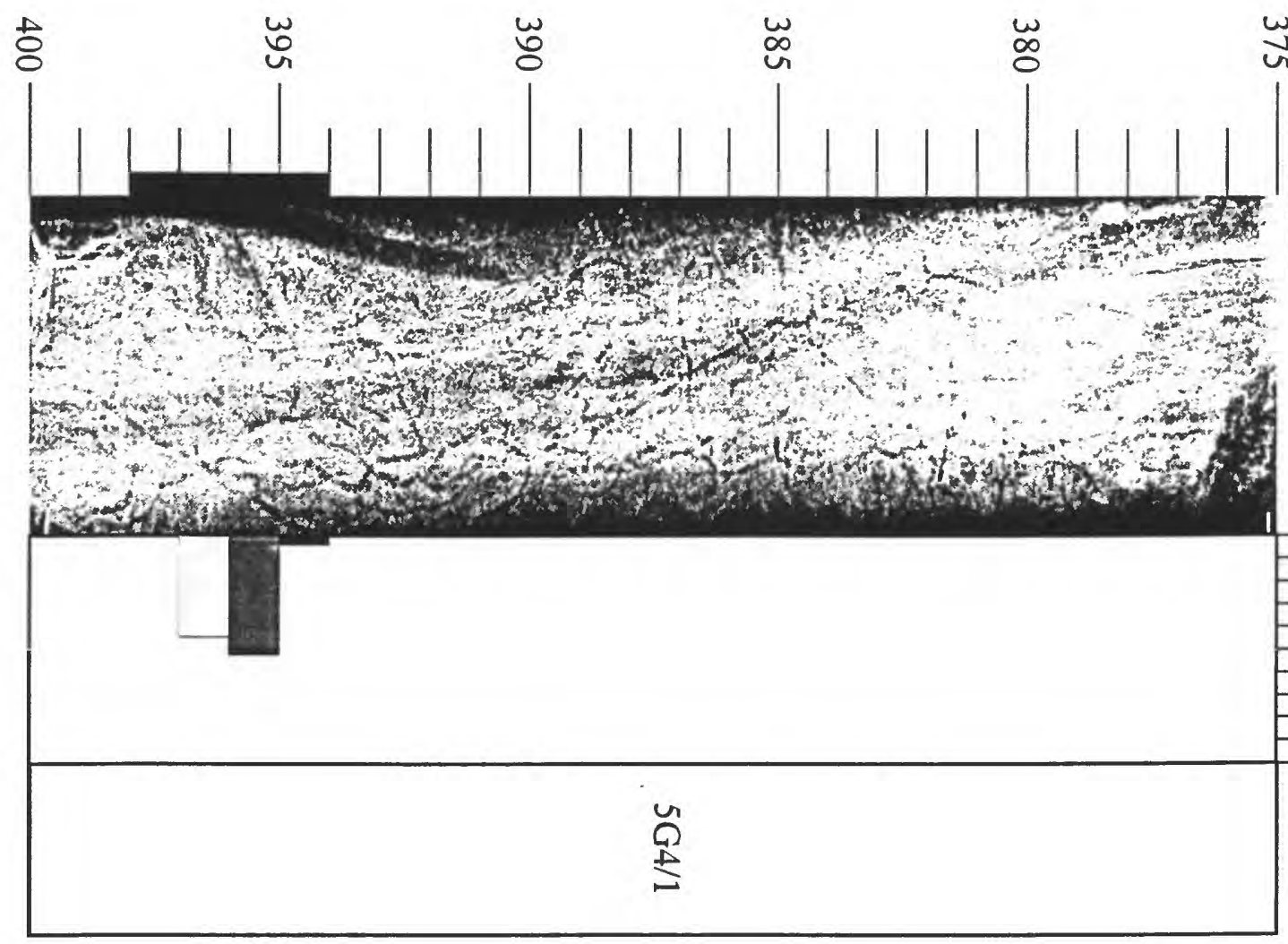

告

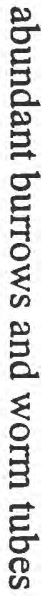

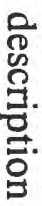




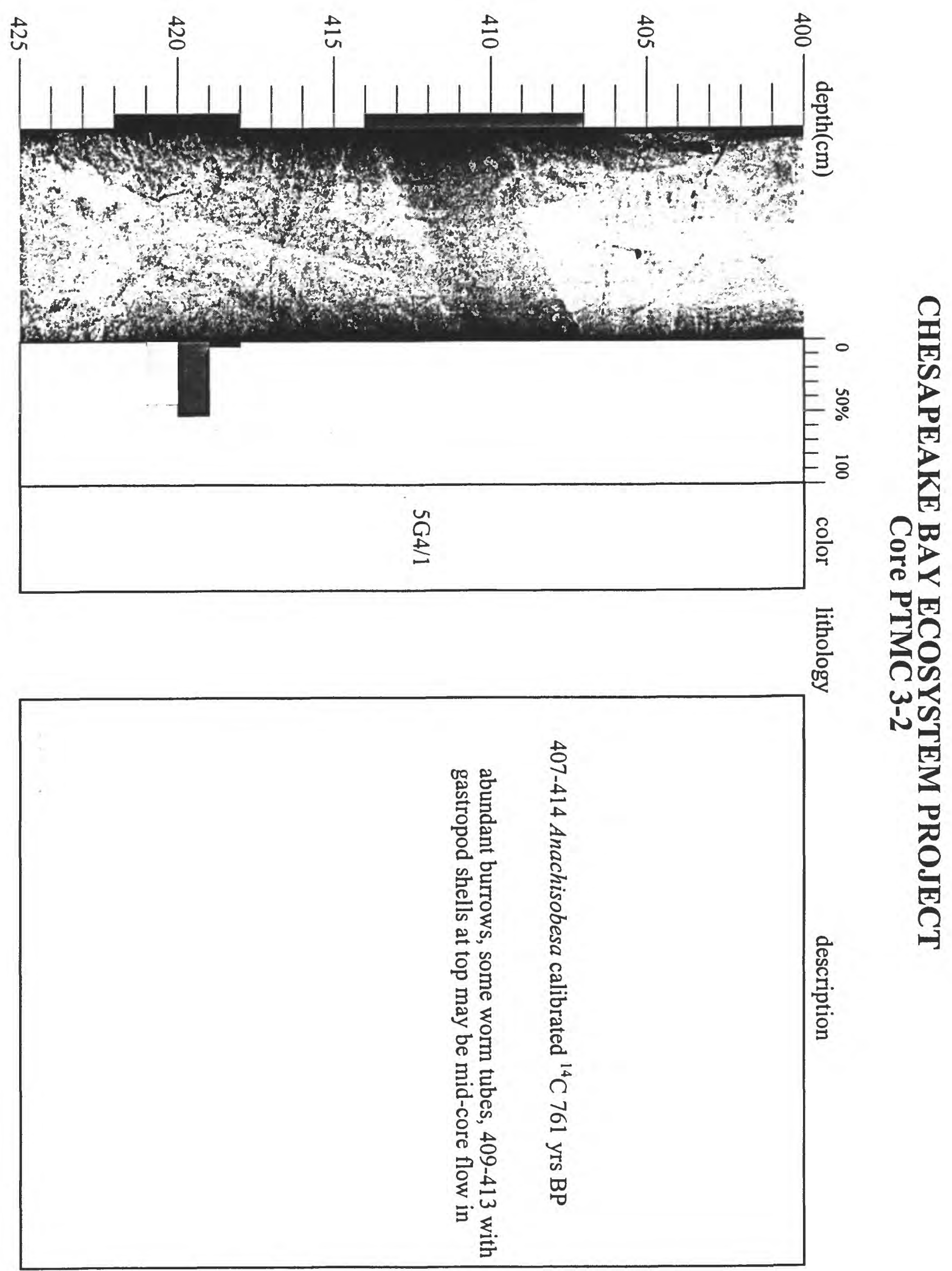




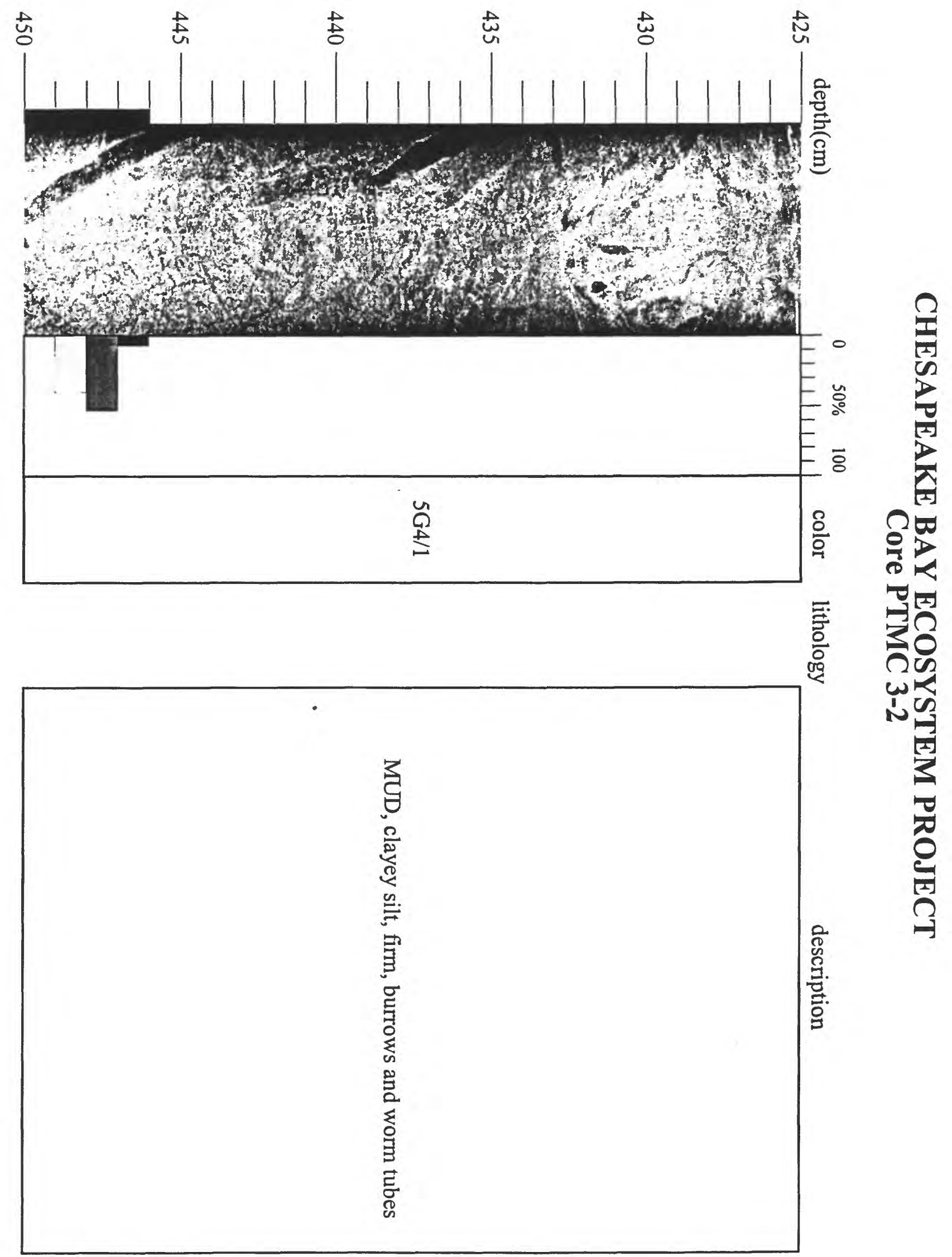




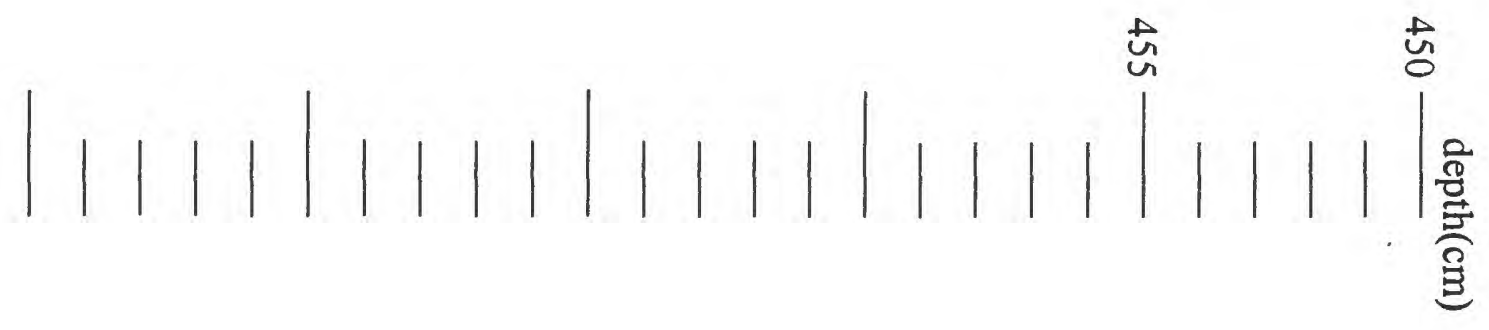

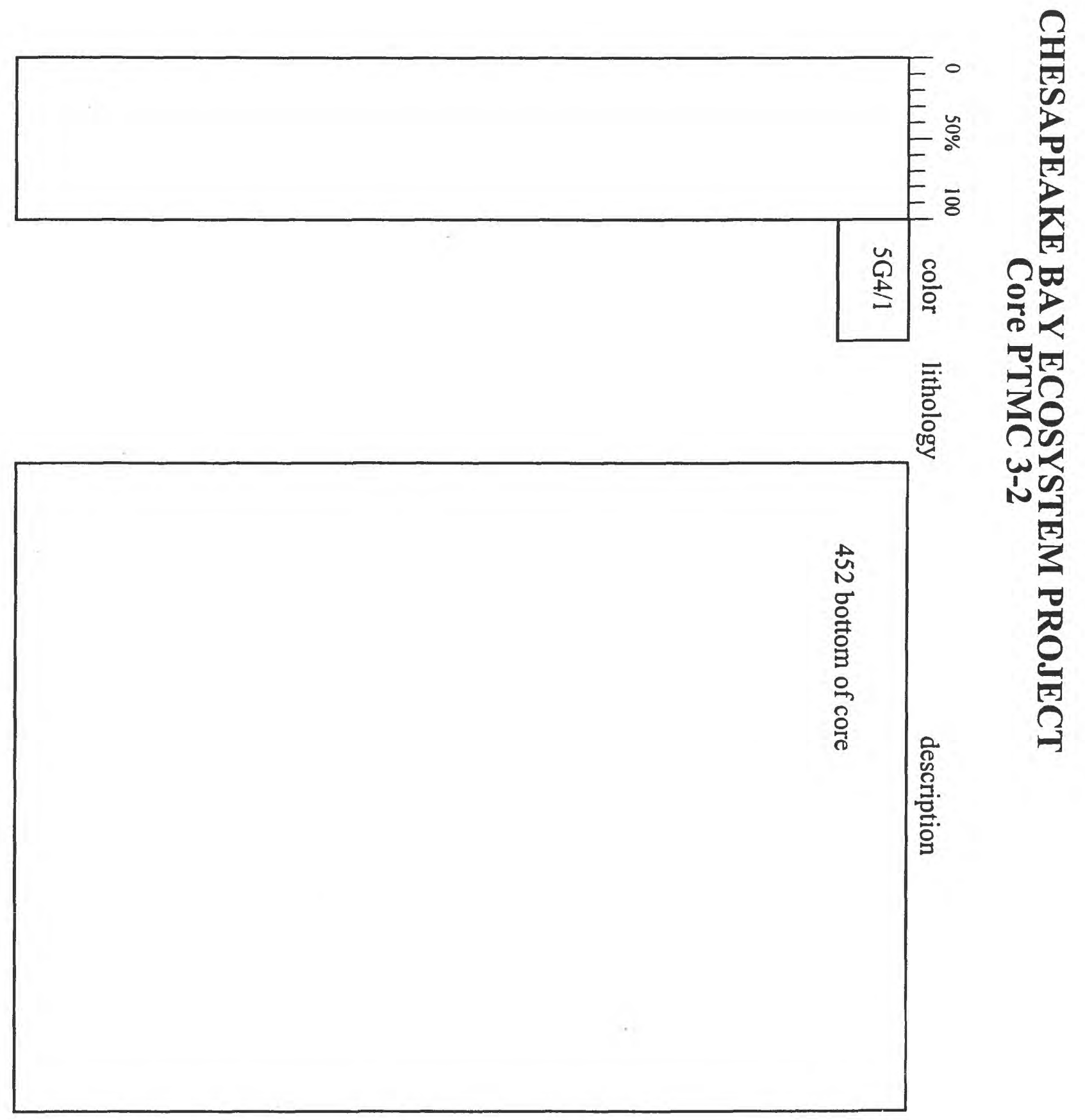

